\title{
The tumour suppressor p53 as a supporter of
}

\section{DNA replication}

\author{
Dissertation \\ for the award of the degree \\ "Doctor rerum naturalium" \\ of the Georg-August-Universität Göttingen \\ within the doctoral program IMPRS Molecular Biology \\ of the Georg-August University School of Science (GAUSS)
}

\author{
submitted by \\ Ina Klusmann \\ from Mülheim an der Ruhr
}

Göttingen 2018 


\section{Thesis Committee}

Prof. Dr. med. Matthias Dobbelstein, Department of Molecular Oncology, University Medical Center, Göttingen

PD Dr. Halyna Shcherbata, Department for Gene Expression and Signaling, Max-PlanckInstitute for Biophysical Chemistry, Göttingen

Prof. Dr. Steven Johnsen, Department for General, Visceral and Pediatric Surgery, University Medical Center, Göttingen

\section{Members of the Examination Board}

Prof. Dr. med. Matthias Dobbelstein, Department of Molecular Oncology, University Medical Center, Göttingen

PD Dr. Halyna Shcherbata, Department for Gene Expression and Signaling, Max-PlanckInstitute for Biophysical Chemistry, Göttingen

\section{Further members of the Examination Board}

Prof. Dr. Steven Johnsen, Department for General, Visceral and Pediatric Surgery, University Medical Center, Göttingen

Prof. Dr. Heidi Hahn, Department of Human Genetics, University Medical Center, Göttingen

Dr. Roland Dosch, Department of Developmental Biochemistry, University Medical Center, Göttingen

Dr. Nuno Raimundo, Department of Cellular Biochemistry, University Medical Center, Göttingen

Date of oral examination: 30th August 2018 
This thesis is dedicated to Arno. 


\section{Contents}

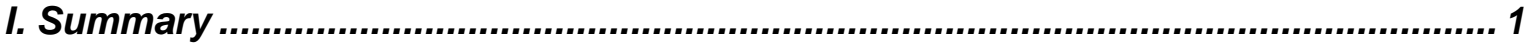

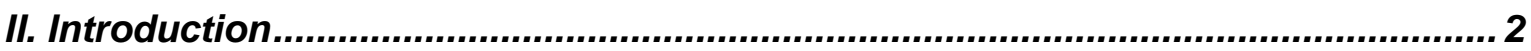

II.1 The p53-MDM2 network ................................................................................. 2

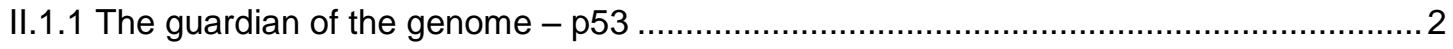

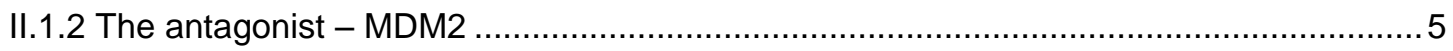

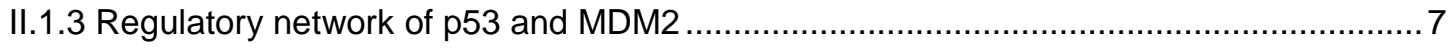

II.1.4 p53-independent functions of MDM2 on genome integrity ......................................

II.1.5 p53-independent functions of chromatin-bound MDM2 …..................................... 10

II.1.6 p53 and MDM2 - guardian and attacker of the genome? .......................................11

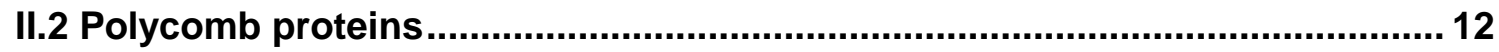

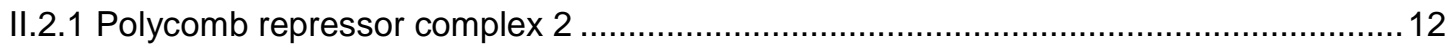

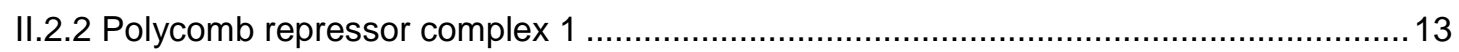

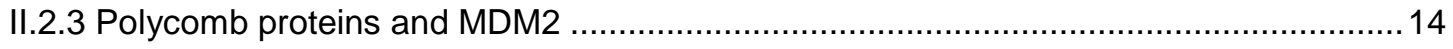

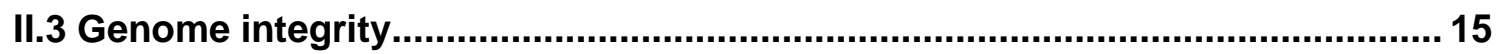

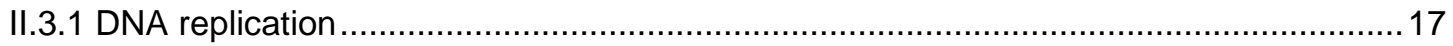

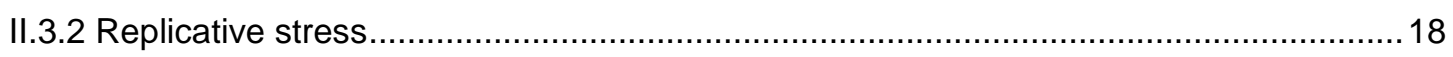

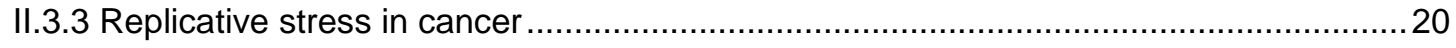

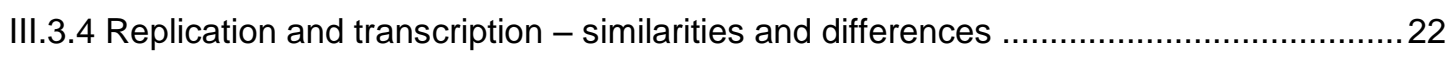

III.3.5 Replication-transcription conflicts - when separation goes wrong .............................23

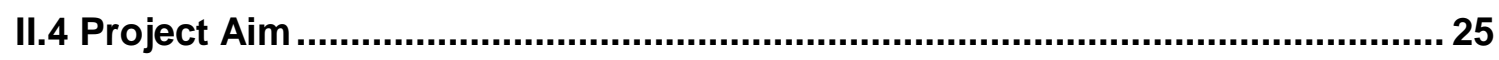

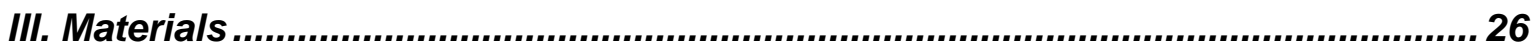

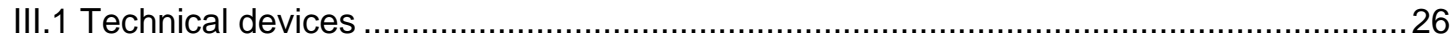

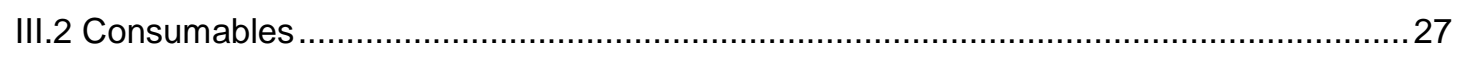

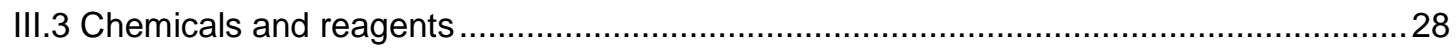

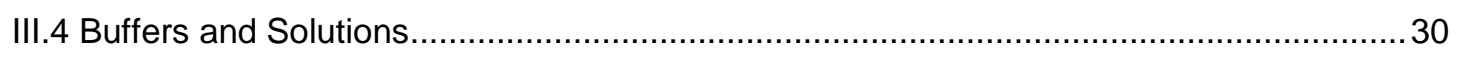

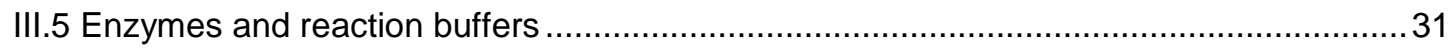

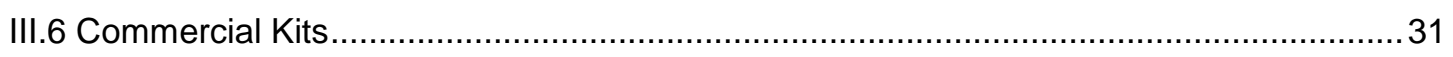


III.7 Chemotherapeutics and Pharmacological inhibitors ................................................. 32

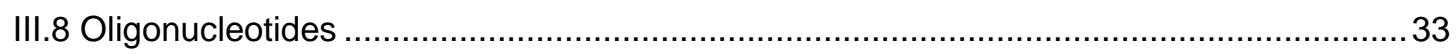

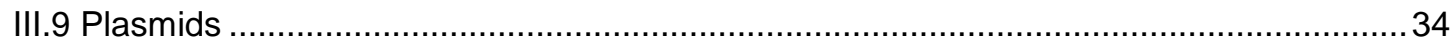

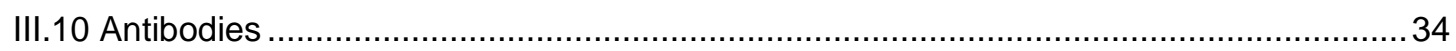

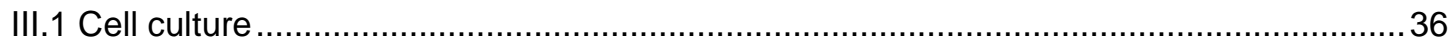

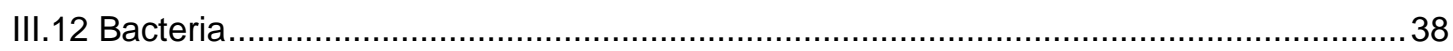

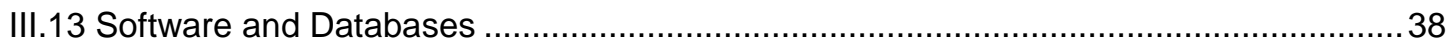

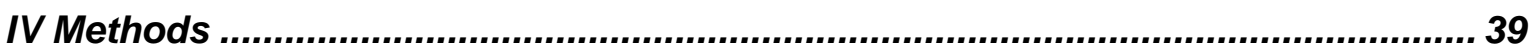

IV.1 Cell Biology ...................................................................................................... 39

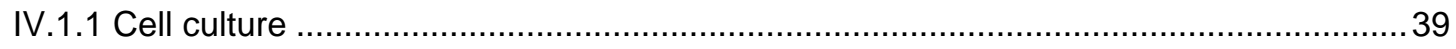

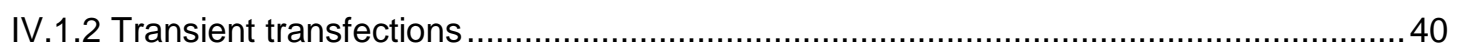

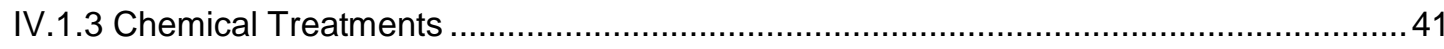

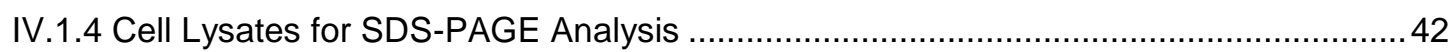

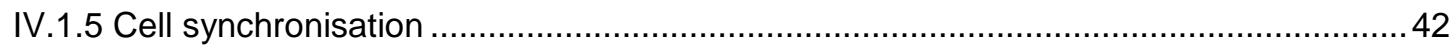

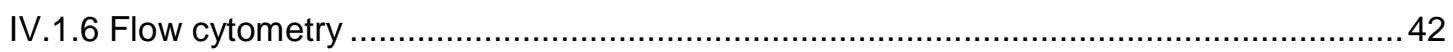

IV.1.7 High-Content Immunofluorescence Microscopy ................................................... 42

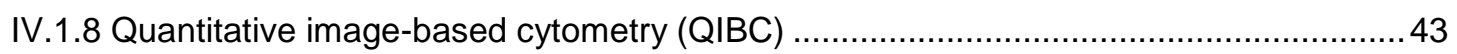

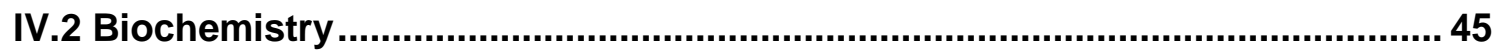

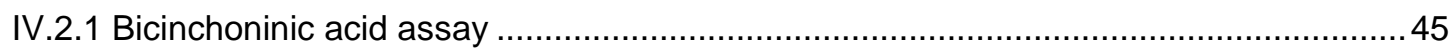

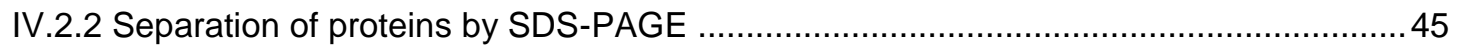

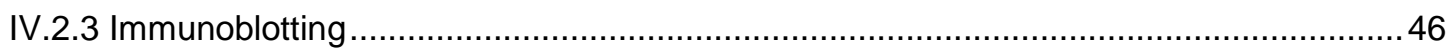

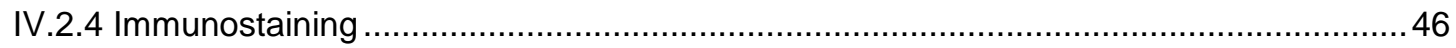

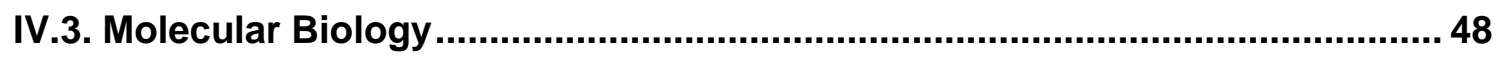

IV.3.1 RNA extraction, reverse transcription, and real time quantitative PCR ....................... 48

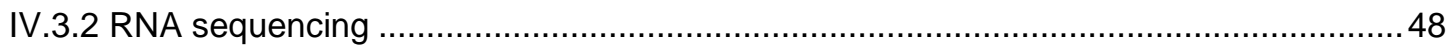

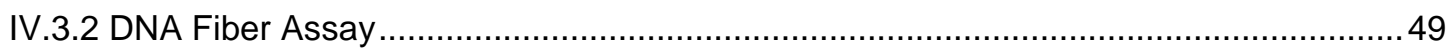

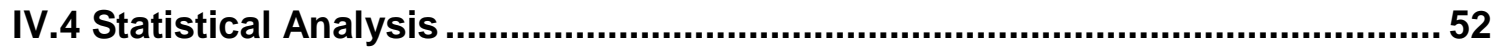


V.I Publication ................................................................................................................. 53

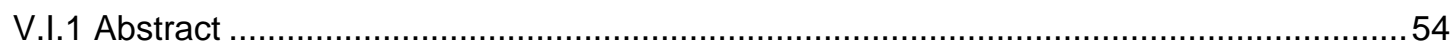

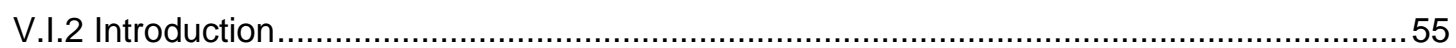

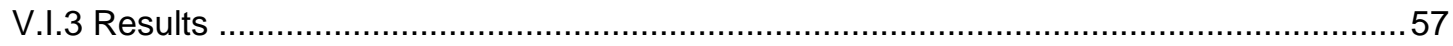

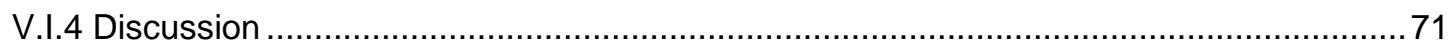

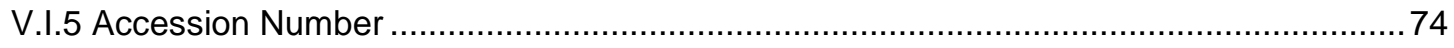

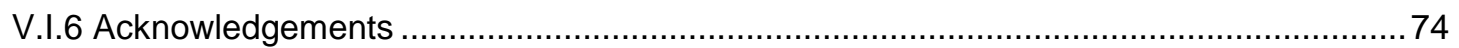

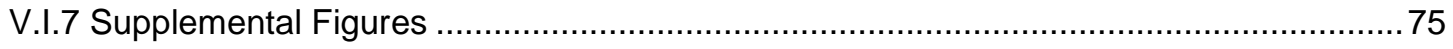

V.II Manuscript

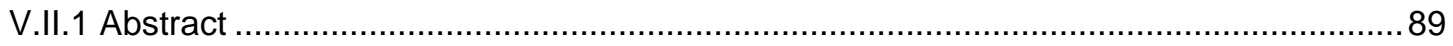

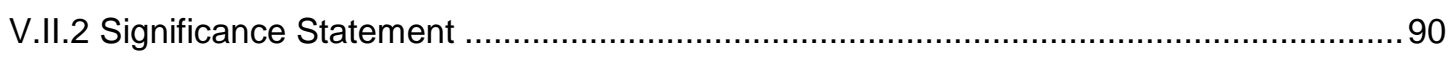

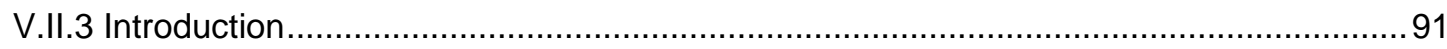

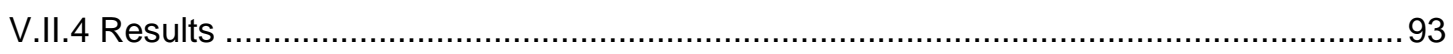

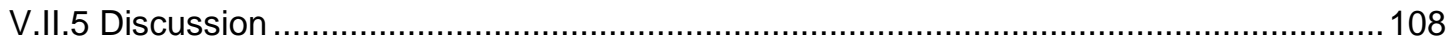

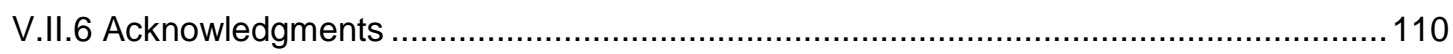

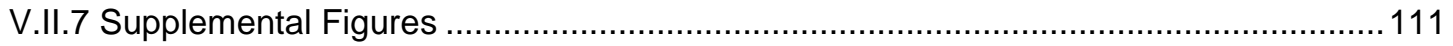

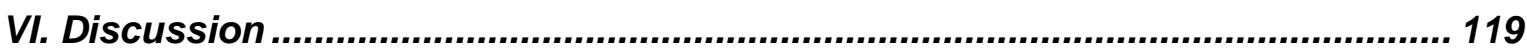

VI. 1 Non-canonical roles of p53 in tumour suppression ................................... 121

VI.1.1 The non-canonical guardian of the genome supports DNA replication .....................122

VI.1.2 A novel strategy to distinguish between fork velocity and fork processivity................ 122

VI.2 Changes in the chromatin landscape can affect replication fork progression

VI.2.1 MDM2 and Polycomb repressor complexes - similarities and differences................. 126

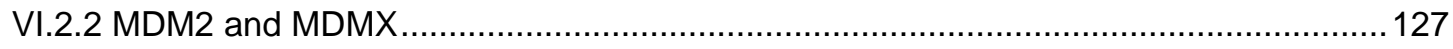

VI.3 R-loops form in the absence of MDM2 ........................................................ 128

VI.3.1 R-loops - natural intermediates and drivers of genome instability ..........................128

VI.3.2 Defects in ribonucleoproteins cause pre-mRNA to thread back into the DNA duplex 130

VI.3.3 Topoisomerases suppress R-loop formation by relaxing torsional stress .................130 
VI.3.4 Ribonucleases and helicases catalyse the removal of R-loops

VI.4 Targeting the p53-MDM2 axis in chemotherapy

VI.5 Conclusions.

VII. References 135

VIII. Acknowledgements 152

IX. Abbreviations 153

X. List of Figures. 155

XI. List of Tables 156

XII. Affidavit. 157

XIII. Curriculum vitae Error! Bookmark not defined. 


\section{Summary}

The tumour suppressor protein p53 is well known for its role in maintaining genetic stability by preventing the propagation of damage onto successive generations of cells. Depending on the extent of damage experienced by a cell, p53 promotes cell cycle arrest to allow for DNA repair or induces apoptosis in cases of severe damage. The canonical functions of p53, commonly referred to as the "guardian of the genome", imply action only after the damage has occurred within a cell. Our work challenges this view by providing evidence pointing to a more direct and pro-active role for p53 in protecting a cellular genome from damage.

p53 activation enhances the processivity of DNA replication and reduces replicative stress, whereas the removal of p53 reduces fork progression. This was observed in tumour-derived cells as well as non-transformed murine embryonic fibroblasts with heterozygous or homozygous p53 deletion, and in freshly isolated thymocytes from mice with differential p53 status. These results expand the tumour-suppressive functions of p53 with an ex-ante activity that prevents DNA damage during replication.

MDM2, target gene and main negative regulator of $p 53$, acts to support DNA replication downstream of p53. In particular, its RING finger domain with intrinsic E3 ubiquitin ligase activity is required for this non-canonical role of MDM2. We demonstrated a mechanism by which MDM2 and its interaction partners of the Polycomb repressor complexes which mediate changes in chromatin compaction, prevent the formation of R-loops on the DNA template that impair replication progression.

The p53-MDM2 axis helps to protect the genome during $S$ phase, by preventing R-loops and hence enhancing replication. Clinically, our study highlights the importance of small molecule inhibitors against MDM2 that inhibit its RING domain on top of inhibitors targeting its interaction with p53 currently evaluated in clinical trials. We propose that the dual inhibition of both these domains of MDM2 using combination treatments would present a more promising chemotherapeutic strategy. 


\section{Introduction}

\section{II.1 The p53-MDM2 network}

\section{II.1.1 The guardian of the genome - p53}

The tumour suppressor protein p53 is encoded by the TP53 gene on chromosome 17p13.1. It is not only subject to the most common mutations in cancers but also functionally attenuated in most tumour entities. p53 was first discovered by coprecipitation of the SV40 T-large antigen in 1979 and has been a major research focus since (Lane and Crawford, 1979; Linzer and Levine, 1979). As a tumour suppressor, p53 acts to protect a cell population from propagating DNA damage onto successive generations of cells and the resulting genomic instability. It is this activity that gave rise to the description of p53 as a "guardian of the genome" by Sir David Lane in 1992 (Lane, 1992). p53 is activated in response to stress signals in the cell and mediates a cellular response in form of gene expression. Depending on the type and extent of stress signals, p53 activation results in cell cycle arrest, DNA repair, changes in metabolism as well as apoptosis.

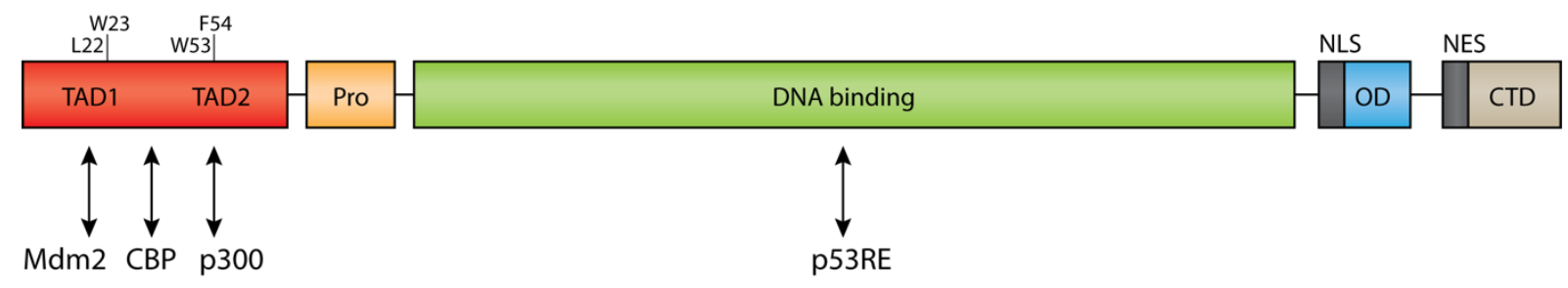

Fig.ll.1.1 Domain structure of p53.

The amino terminal portion of p53 protein consists of two transactivation domains (TADs). The most critical residues for its activity are highlighted above. A proline-rich domain (Pro) as well as the DNA binding domain that interacts with response elements (p53RE) on template DNA are located at the core of the protein. The carboxyl terminus consists of an oligomerisation domain (OD) harbouring a nuclear localisation signal (NLS) and a C-terminal domain (CTD) with a nuclear export signal (NES). 
The TP53 gene locus encodes for a multi-domain protein with a molecular weight of about $53 \mathrm{kDa}$. It is heavily post-translationally modified for both its activation and degradation resulting in a fast turnover and short half-life. In its activated form, p53 tetramerises and acts as a transcription factor to induce target gene expression. Several features of its domain structure reflect its function as a transcription factor (Fig.II.1.1).

The most amino terminal region of the protein is highly acidic and contains interspersed hydrophobic residues. This region ranging from aa 1 to 83 can be further subdivided into two distinct transactivation domains (TADs) that act synergistically to bind to transcriptional co-regulators (Candau et al., 1997; Chang et al., 1995). Most critical are not the acidic but specific hydrophobic residues within these domains that allow binding to factors involved in histone modifications, chromatin remodelling as well as transcription initiation and elongation (Lemon and Tjian, 2000). Studies using knock-in mutants for both TADs have identified distinct TAD-dependent target gene induction. A co-disruption of active residues in both TADs led to loss of transactivation and tumour suppression capacities (Raj and Attardi, 2017). For transactivation, p53 directly interacts with TATAbinding protein (TBP) and associated factors involved in pre-initiation complex (PIC) formation at the gene promotor but also with histone-acetyl-transferases p300 and CREBbinding protein (CBP) that mediate chromatin opening. Kinases involved in the cellular stress response phosphorylate p53 at seven residues within the TADs (Jenkins et al., 2012). These modifications stabilise p53 by preventing the interaction with its negative regulator MDM2 but also by enhancing its affinity for transcriptional co-factors such as TFIIH, CBP and p300 (Teufel et al., 2009).

A proline-rich domain (amino acids 60-90) can also be found in the N-terminus of p53. It consists of five repeats of the PXXP motif and was found to play a role in p53-mediated apoptosis (Baptiste et al., 2002).

A DNA binding domain spanning from aa residues 102 to 292 forms the central core of p53. This domain specifically binds to p53 response elements (p53RE) in a sequence specific manner. The motif is composed of two palindromic "half-sites" that are separated by spacer sequences of up to 13 nucleotides (Kawamura et al., 2009; Smeenk et al., 2008). A recent study has identified that $p 53$ tetramers can bind to one half site of the motif on the DNA template, allowing two tetramers to bind to one response element at a time. The resulting DNA loops facilitate transcriptional activation (Kearns et al., 2016). The majority of p53 mutations found in cancer cells are located within the DNA binding domain. Only about $10 \%$ of all are nonsense mutations or deletions with no protein being 
produced. The remaining $90 \%$ consist of various missense mutations of which some are more prominent, independent of the tumour entity (so called "hotspot mutations"; Baugh et al. 2017). Missense mutations alter the amino acid sequence and influence either DNA binding affinity or conformation of the protein. On the other hand, many missense mutations also lead to a novel function ("gain-of-function") that presents a selective advantage in terms of cell proliferation, migration or other cancer cell specific characteristics (Dittmer et al., 1993; Oren and Rotter, 2010). Mutant p53 molecules exert a dominant negative effect on their wildtype counterparts so that tetramers that contain only one mutant subunit are non-functional in terms of transactivating p53-target genes (Willis et al., 2004).

The carboxyl terminus of p53 harbours an oligomerisation domain from aa 325 to 356 . A monomer of the oligomerisation (or tetramerisation) domain forms a $\beta$-sheet and an $\alpha$ helix linked by a single glycine residue (Chène, 2001). Structural data suggests that tetramers form as dimer of dimers by the interaction of two $\beta$-sheets to form an antiparallel double-sheet and subsequently interacts with a double-helical bundle (Lee et al., 1994; Mateu and Fersht, 1999; Mateu et al., 1999).

In addition, p53 contains an unstructured and basic C-terminal domain (CTD) at aa 363 to 393 as well as both a nuclear localisation and export signal. The CTD of p53 has regulatory function and is an intrinsically disordered domain (IDD) that can adopt several conformations upon binding to different interaction partners. So far, it has been found to form $\alpha$-helices, $\beta$-strands, $\beta$-turns and $U$-shapes, providing a mechanism for regulatory diversity (Sullivan et al., 2017). The CTD inhibits the DNA binding domain in its unmodified state and is post-translationally modified for its activation (Friedler et al., 2005). 


\section{II.1.2 The antagonist - MDM2}

The oncogenic murine double minute $2(m d m 2)$ gene was first identified in a screen for amplified DNA sequences in a spontaneously transformed murine BALB/c cell line in 1987 and is now mainly known for its regulatory role towards the tumour suppressor p53 (Cahilly-Snyder et al., 1987). Double minutes are small extrachromosomal chromatin particles that divide in the absence of centromeres (Barker, 1982). Genes for mdm1-3 were found expressed at 50-fold level in the 3T3DM cell line it was identified in, however, only overexpression of MDM2 was sufficient to transform two non-tumorigenic cell lines (Fakharzadeh et al., 1991).

Both the murine mdm2 and the human MDM2 gene contain 12 exons and encode for two different forms of the MDM2 protein from two promotors. The first promotor (P1) expresses a long version of the protein ( $\mathrm{p} 90^{\mathrm{MDM} 2}$ ) and is involved in negatively regulating $\mathrm{p} 53$, whereas the shorter version expressed from P2 (p76 ${ }^{\mathrm{MDM} 2}$ ) lacks the p53 binding domain and cannot cause p53 degradation. On the other hand, P2 is a p53-responsive promotor and its shorter product acts as a dominant negative inhibitor on $590^{\mathrm{MDM} 2}$ resulting in an accumulation of p53 (Perry et al., 2000). Alternative splicing adds to the variety of isoforms of MDM2 in tissues and tumours (Iwakuma and Lozano, 2003). In both murine and human cell lines, most transcripts are expressed from P1 to produce full length MDM2 protein (Barak et al., 1994; Mendrysa and Perry, 2000).

Human MDM2 is a protein of 491 amino acids in length and shows a distinct domain structure (Fig. II.1.2). Its amino terminal domain forms a small hydrophobic pocket (aa 25109) that interacts with an amphipathic $\alpha$-helix present in the amino terminal portion of p53 (Kussie et al., 1996). In addition, MDM2 can also bind to the E2F1 transcription factor to prevent its proteasomal degradation (Zhang et al., 2005).

A nuclear localisation signal (NLS) and a nuclear export signal (NES) encoded by aa 178192 as well as a nucleolar localisation signal within the C-terminal RING domain allow a regulated nuclear-cytoplasmic shuttling of the protein important for p53-dependent and independent functions of MDM2 (Roth et al., 1998).

The central acidic (aa 237-288) and zinc finger domain (aa 289-331) are located towards the centre of the polypeptide and regulate p53 activity in two ways. On one hand, they repress DNA damage induced and sequence specific transactivation of p53 by binding to and inhibiting p300/CBP-p53 complex formation (Kobet et al., 2000). On the other hand, 
the acidic domain was also found to directly mediate p53 degradation in a RINGindependent fashion (Argentini et al., 2001).

The C-terminal part of MDM2 consists of a "really interesting new gene" (RING) domain (aa 438-491) that has intrinsic E3 ubiquitin ligase activity. RING domains typically contain seven cysteines and one histidine to coordinate two zinc cations required for catalysis (Borden and Freemont, 1996). This feature can also be found in MDM2 in the form of two zinc binding sites: (I) Cys ${ }^{438}$, Cys ${ }^{441}, \mathrm{Cys}^{461}$, Cys ${ }^{464}$ and (II) $\mathrm{His}^{452}, \mathrm{His}^{457}$, Cys ${ }^{475}$, Cys ${ }^{478}$ (Shloush et al., 2011). As an E3 ubiquitin ligase, MDM2 can transfer ubiquitin moieties from an E2 ligase like UBE2D1 onto target proteins to mark them for degradation by the proteasome. The most prominent target of MDM2 ubiquitination is p53, but it can also transfer ubiquitin onto other proteins including itself depending on the cellular signals conveyed to it via post-translational modifications (Fang et al., 2000; Honda et al., 1997).

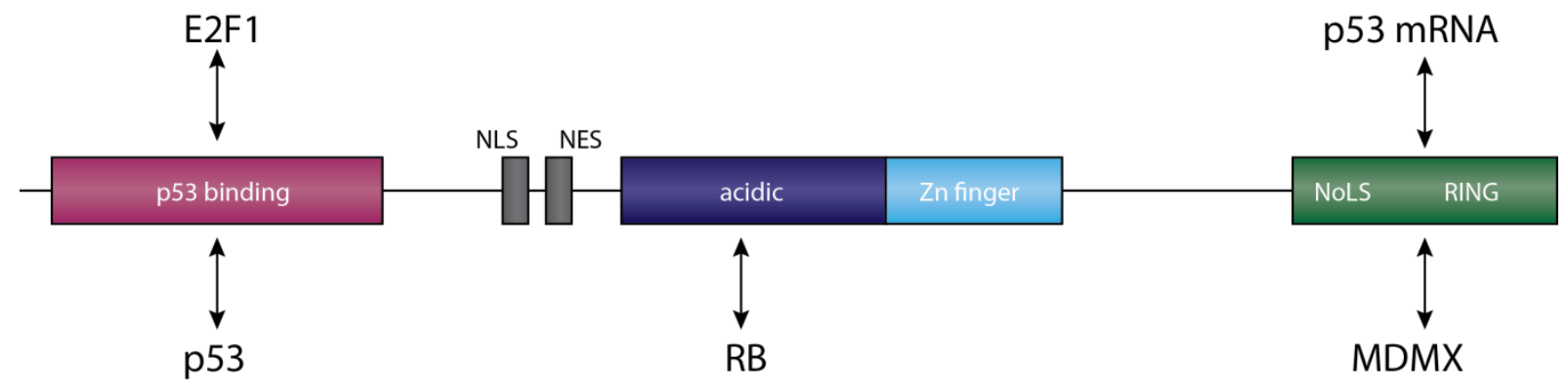

Fig.II.1.2 Domain architecture of MDM2.

The MDM2 protein consists of an N-terminal p53-binding domain, a nuclear localisation and export signal, an acidic domain, a Zinc ( $\mathrm{Zn}$ ) finger domain as well as a C-terminal RING domain harbouring a nucleolar localisation signal.

A structurally related protein to MDM2 called MDMX (or MDM4) was identified as a p53 binding partner in 1996 and is thought to have evolved by gene duplication over 440 million years ago (Momand et al., 2011; Shvarts et al., 1996). The MDMX gene is located at chromosomal location $1 \mathrm{q} 32$ and encodes for a 490 amino acid long protein. MDMX shares a high sequence homology with MDM2 and contains similar domain architecture. Like MDM2, it contains a p53 binding domain in its $\mathrm{N}$-terminus as well as a C-terminal RING finger. Unlike MDM2, the RING domain in MDMX does not contain E3 ubiquitin ligase activity but it is thought to act as an E4 protein supporting the addition of ubiquitin chains to monoubiquitinated substrates (Wang et al., 2011). With regard to p53, MDMX inhibits p53 transactivation, whereas MDM2 can also cause proteasomal degradation of 
p53 via ubiquitination (Marine et al., 2006). MDM2 and MDMX can form heterodimers that prevent auto-ubiquitination of MDM2 to increase its stability and its negative regulation towards p53 (Sharp and Kratowicz, 1999; Tanimura et al., 1999).

\section{II.1.3 Regulatory network of p53 and MDM2}

In unstressed cells, levels and activity of p53 are kept under tight control by a number of mechanisms including intracellular localisation, protein-protein interactions as well as post-translational modifications (Lavin and Gueven, 2006).

The MDM family proteins MDM2 and MDMX are the main negative regulators of p53 in the cell. Both MDM proteins can interact with the $\mathrm{N}$-terminus of $\mathrm{p53}$, masking its transactivation domain thereby inhibiting its function (Momand et al., 1992; Oliner et al., 1993). A more recent study has also described a conformational change in p53 by the acidic domain of MDM2 causing p53 to lose DNA binding ability, thus further impairing its activity as a transcription factor (Cross et al., 2011).

In addition, MDM2 and MDMX form heterodimers via their RING domains resulting in a more stable and active MDM2 that ubiquitinates p53. At low levels of MDM2, monoubiquitination of six C-terminal lysine residues on p53 exposes a nuclear export signal in its oligomerisation domain resulting in its cytoplasmic translocation ( $\mathrm{Li}$ et al., 2003; Lohrum et al., 2001). At high levels of MDM2, p53 is marked for proteasomal degradation by polyubiquitin chains (Haupt et al., 1997; Honda et al., 1997; Li et al., 2003).

The different mechanisms by which MDM2 regulates p53 are further complicated by the establishment of a negative feedback loop between the two proteins (Fig.II.1.3). MDM2 is not only a negative regulator of p53 stability and activity but also a transcriptional target of active p53 tetramers (Wu et al., 1993).

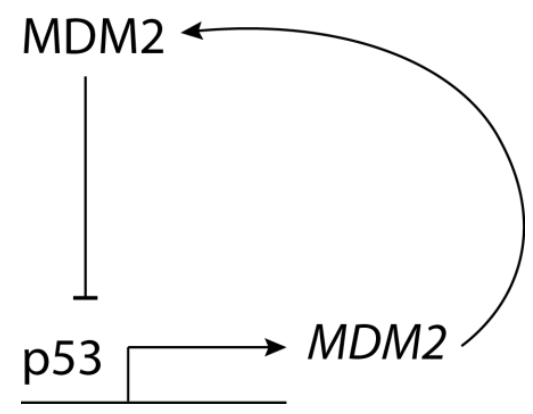

Fig.II.1.3 The auto-regulatory feedback loop of p53 and MDM2.

The stability and activity of the tumour suppressor p53 is regulated by MDM2. An activation of p53 induces expression of the target gene MDM2 and the increase of MDM2 protein levels mark p53 for proteasomal degradation. 
In response to genotoxic stress in a cell, p53 is stabilised and activated as a transcription factor (Fig.II.1.4). Mediator kinases ATM, ATR, and CHK1\&2 activated in response to damage, phosphorylate p53 at several residues, most of which are found in its $\mathrm{N}$-terminus (e.g. Ser15 and Ser20). These modifications inhibit the interaction between p53 and its negative regulators MDM2 and MDMX. In the absence of this repression, p53 forms homotetramers via its oligomerisation domain and binds to p53 response elements in target gene promotors (reviewed in Vogelstein et al. 2000; Horn \& Vousden 2007; Bieging et al. 2014).

In addition, phosphorylation of MDM2 at Ser395 and MDMX at Ser403 (near the RING domain) by the mediator kinase ATM switch them from negative to positive regulators of p53, promoting p53 mRNA translation and proper folding of the nascent peptide (Gajjar et al., 2012; Malbert-Colas et al., 2014).

1)

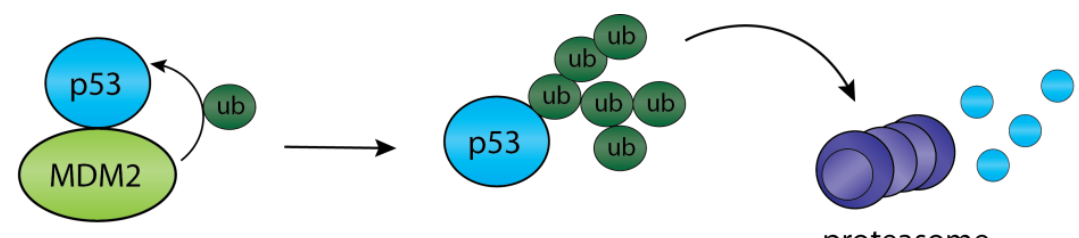

proteasome

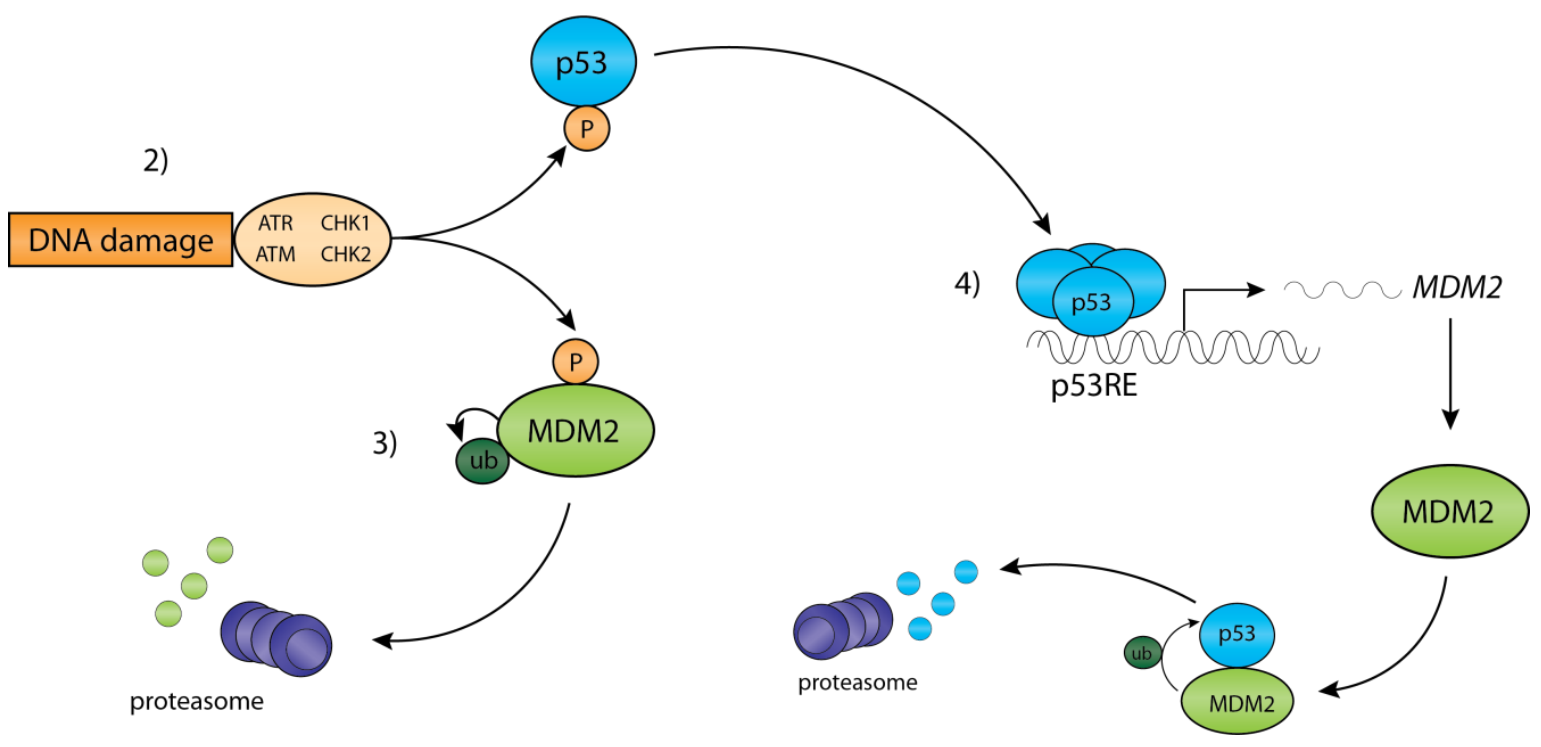

Fig.II.1.4 The p53 and MDM2 regulatory network.

(1) In the absence of stress signals, p53 is kept at very low levels by active MDM2 (and MDMX, not shown for simplification). Under genotoxic stress conditions, MDM2 is inactivated (2) and p53 can induce target gene expression in the nucleus (3). In order to return to basal levels quickly, a negative feedback loop is in place. (4) In response to active p53, MDM2 is expressed and if not inactivated by damage signalling kinases, it can continue to inhibit p53 activity and stability.

In addition to activating phosphorylations, p53 is further modified by acetylation, sumoylation, neddylation, methylation, and ubiquitination. The variety and combination of different modifications is thought to provide a "code" that allows suitable transcriptional 
programs depending on the type of damage experienced by a cell (Meek and Anderson, 1994). This mechanism is best seen in the example of DNA damage signalling. Upon genotoxic stress, the DNA damage signalling cascade activates p53 as described above. Under mild stress conditions, p53 induces the expression of transcriptional targets that halt the cell cycle. The gene product of $C D K N 1 A, \mathrm{p} 21$, is a potent CDK inhibitor that arrests cells in G1 phase of their cell cycle, wheras another product called 14-3-3- $\sigma$ induces a $\mathrm{G} 2$ arrest by inhibiting the $\mathrm{CDC} 25 \mathrm{C}$ phosphatase required for progression into mitosis (Harper et al., 1995; Hermeking et al., 1997).

The transcriptional program in response to severe damage is very different and the cell undergoes programmed cell death. The pro-apoptotic transcriptional program of p53 induces the expression of target genes like Puma, Noxa, and Bax that trigger the release of cytochrome c from mitochondria. Released cytochrome $\mathrm{c}$ binds to the p53-targets Apaf and caspase 9 forming an apoptosome that initiates the caspase cascade for apoptosis initiation (Rozenfeld-Granot et al., 2002).

\section{II.1.4 p53-independent functions of MDM2 on genome integrity}

Although the best known role of MDM2 is to regulate p53 on various levels, more evidence for p53-independent roles of MDM2 have emerged. First hints for these roles were observed in tumours lacking p53 but nevertheless overexpressing MDM2, as well as the existence of splice variants of MDM2 that are unable to bind to p53 but capable of transforming cells (Cordon-Cardo et al., 1994; Lu et al., 2002; Sigalas et al., 1996). Since then, MDM2 has been described to play a role in various processes, some of which will be mentioned here (Jain and Barton, 2016; Li and Lozano, 2013).

The overexpression of MDM2 in a cell causes genomic instability by inhibiting DNA repair and suppressing cell cycle arrest regardless of its p53 status. Evidence for increased genomic instability was found by overexpressing MDM2 in fibroblasts as well as in transgenic mice in the form of increased chromosomal abnormalities as well as aneuploidy (Jones et al., 1998; Lushnikova et al., 2011).

In 2005, Alt et al. analysed interaction partners of endogenous MDM2 in a mass spectrometry approach and precipitated all three components of the MRN complex involved in DNA repair: Nbs1, Mre1, Rad50. Through a direct interaction with the Nbs1 component, MDM2 localises to sites of DNA damage which in turn dampens DNA damage signalling and delays repair of the damaged site mediated by the MRN complex (Alt et al., 2005; Eischen, 2017). 
In addition to its role in modulating DNA repair, MDM2 can also influence cell cycle progression independently of p53. A direct interaction of MDM2's acidic domain (aa 254264) and the C-terminal portion of retinoblastoma protein (RB; aa 785-803) prevents the interaction of RB with E2F1 via the same domain (Sdek et al., 2004; Xiao et al., 1995). RB binds to several E2F transcription factors to inhibit their cell cycle specific transcriptional program. By sequestering RB and stimulating E2F1, MDM2 relieves a block on cell cycle progression in an unscheduled manner and causes genomic instability by missegregation of chromosomes in the following mitosis (Hernández-Monge et al., 2016; Martin et al., 1995). Additionally, MDM2 can also mark RB as well as other cell cycle inhibitors like p21 and hnRNP K for proteasomal degradation (Bouska and Eischen, 2009; Li and Lozano, 2013; Uchida et al., 2005).

A third pathway MDM2 can act on independently of p53 is apoptosis. MDM2 levels are inversely correlated with the forkhead transcription factor FOXO3a in breast cancer cells due to active degradation of FOXO3a by MDM2 (Yang et al., 2008). By this, MDM2 counteracts FOXO3a-mediated apoptosis and allows tumour progression. Another level of suppressing apoptosis mediated by MDM2 is by enhancing the translation of the antiapoptotic XIAP (Gu et al., 2009).

\section{II.1.5 p53-independent functions of chromatin-bound MDM2}

More recently, two different p53-independent roles for transcriptional regulation have been described for chromatin-bound MDM2 (Jain and Barton, 2016).

On one hand, MDM2 is required for stemness maintenance in the induction of pluripotent stem cells (iPSCs), osteoblasts, as well as for the proliferative capacity of cancer cells. Mechanistically, MDM2 seems to interact with the chromatin modifying complexes of the Polycomb group to repress target gene expression in a RING-dependent manner (Wienken et al., 2016).

Another group showed that MDM2 has an increased half-life of more than three hours in its chromatin-bound state and that the acidic domain within MDM2 is required for chromatin interactions. In this study, chromatin recruitment of MDM2 is shown to regulate transcriptional programs involved in serine metabolism as well as redox homeostasis (Riscal et al., 2016). 


\section{II.1.6 p53 and MDM2 - guardian and attacker of the genome?}

The tumour suppressor p53 acts to protect the genomic integrity of a cell in various ways. A plethora of different post-translational modifications and the ability to induce a large repertoire of different target genes allow p53 to react to cellular stress with a suitable response. This ensures that the overall population of cells retain their genome integrity by repairing lesions when possible or eliminating damaged cells before they can divide any further. In a normal cell, p53 activity is tightly regulated by MDM2 (and other pathways not discussed here) to ensure an immediate and short response only when necessary.

MDM2 on the other side has been described as a hub-oncogene due to its many interaction partners and regulatory functions (Fåhraeus and Olivares-Illana, 2014). To achieve such a large number of different interactions (some of which were mentioned in this chapter), MDM2 is present in several isoforms due to differential promotor usage as well as alternative splicing and is also heavily post-translationally modified. Untransformed cells contain only very low levels of MDM2 in the absence of stress signalling. In tumours on the other hand, MDM2 is often amplified and expressed at high levels and associated with genome instability (Bouska et al., 2008; Momand et al., 1998).

It seems like the balance of p53 and MDM2 is essential in maintaining genome integrity and is often disrupted in transformed cells by a mutation in p53 or the upregulation of MDM2 but it remains unclear how direct this effect is. Both p53 and MDM2 are known to interact with DNA/chromatin and could have more direct effects on the genome of a cell.

Genome integrity is at highest risk during DNA synthesis in the S-phase of the cell cycle. So far, not much is known about the role of p53 and MDM2 after the cell passes the G1/S checkpoint, however some evidence exists that p53 has altered transactivation ability in S-phase under stress conditions (Gottifredi et al., 2001). 


\section{II.2 Polycomb proteins}

Polycomb group (PcG) proteins were first identified as developmental mutants in Drosophila with segmentation defects similar to the Polycomb mutant described in the late 1940s (Jürgens, 1985; Lewis, 1978, 1949). The importance of these genes in development was further highlighted by the finding that the deletion of many of these genes causes early embryonic lethality in mice (Faust et al., 1995; O'Carroll et al., 2001; Pasini et al., 2004). It is now known that PcG proteins in Drosophila, as well as their highly conserved counterparts in vertebrates, act as negative regulators of developmental transcription factors of the Hox clusters as well as many other target genes involved in development (Boyer et al., 2006; McKenzie Duncan, 1982; Schwartz et al., 2006).

PcG proteins are commonly found in multiprotein complexes including the Polycomb repressor complexes 1 (PRC1) and 2 (PRC2). Both complexes act as negative regulators on target gene expression by altering the chromatin structure around the transcriptional start sites via histone modifications. The PRC2 complex is thought to mediate di- or trimethylation of histone 3 at lysine 27 (H3K27me2/3) and the PRC1 complex monoubiquitinates histone 2A at lysine residue 119 (H2AK119ub1; Schuettengruber and Cavalli, 2009).

\section{II.2.1 Polycomb repressor complex 2}

Polycomb repressor complex 2 consists of four core proteins in both Drosophila and mammals: the catalytic subunit EZH1 or EZH2 and the regulatory subunits SUZ12, EED, and RbAp46/48. Accessory proteins like JARID2, AEBP2, and PCL interact with core components in a transient manner and additionally regulate recruitment as well as activity of the complex (Fig.II.2.1; Margueron \& Reinberg 2011).

The catalytic subunits EZH1 and 2 have methyltransferase activity in their SET domain and can mediate both di- and trimethylation reactions. EZH1 can be found in both dividing and differentiated cells, whereas EZH2 is only present in proliferating cells. However, $\mathrm{EZH} 1$ has a much lower methyltransferase activity compared to EZH2 and is thought to be responsible for re-establishing histone marks after histone exchanges or demethylation, whereas EZH2 mediates methylation reactions in response to cellular signalling (Margueron et al., 2008). 


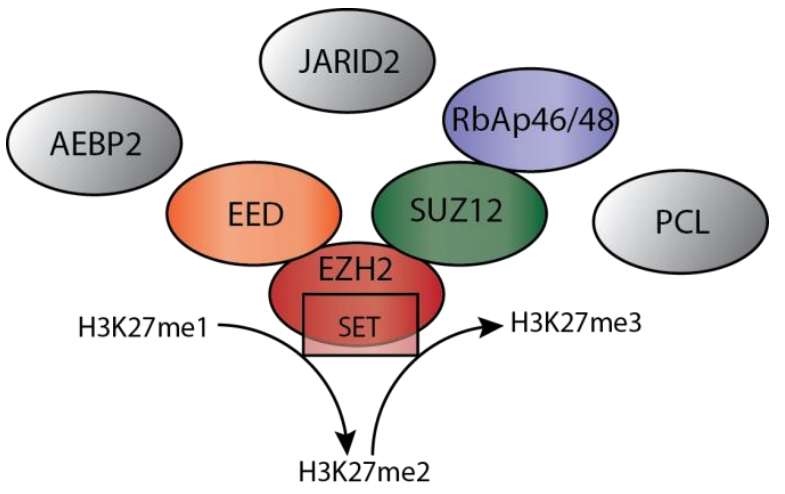

Fig.Il.2.1 Polycomb repressor complex 2.

Mammalian core components of the PRC2 complex highlighted in colour: The catalytic subunit EZH2 contains a SET domain for methyltransferase activity. Regulatory subunits EED, SUZ12, and RbAp46/48 can interact with accessory components JARID2, AEBP2, and PCLs to further modify PRC2 recruitment and target gene specificity.

The methylation of $\mathrm{H} 3 \mathrm{~K} 27$ is processive so that a trimethylation is catalysed by a monomethylation of H3K27me2. Monomethylated H3K27 is a mark for constitutive heterochromatin that is more stable in its repression and usually found in gene-poor regions of the genome. Both di- and trimethylated H3K27, are associated with facultative heterochromatin, a compact region of chromatin that is regulated by developmental cues (Trojer and Reinberg, 2007; Zee et al., 2010).

\section{II.2.2 Polycomb repressor complex 1}

Polycomb repressor complex 1 composition in mammals is more complex than the one of PRC2 and is rather a family of complexes (Schuettengruber et al., 2007). The core proteins identified in Drosophila (Polycomb, Polyhomeotic, Posterior sex combs, and dRing) have at least two homologues each (Levine et al., 2002; Shao et al., 1999). In mammalian cells, the catalytic component RNF2 (or RING1B) is an E3 ubiquitin ligase that is responsible for the monoubiquitination of $\mathrm{H} 2 \mathrm{~A}$ at lysine 119 , a histone mark that silences target genes (Wang et al., 2004). Interaction partners of RNF2 within the PRC1 complex include the RING finger proteins BMI1, MEL18, and NSPC1 (Fig.Il.2.2; Simon \& Kingston 2009).

CBX proteins, homologues to the Drosophila Polycomb, contain a chromodomain that can specifically recognise trimethylated H3K27 (Fischle et al., 2003). Even though PRC1 and PRC2 share many target genes, it is now believed that recruitment of PRC1 does not depend on prior activity of PRC2 but rather on response elements in the target gene promotor (Margueron and Reinberg, 2011; Sing et al., 2009). 


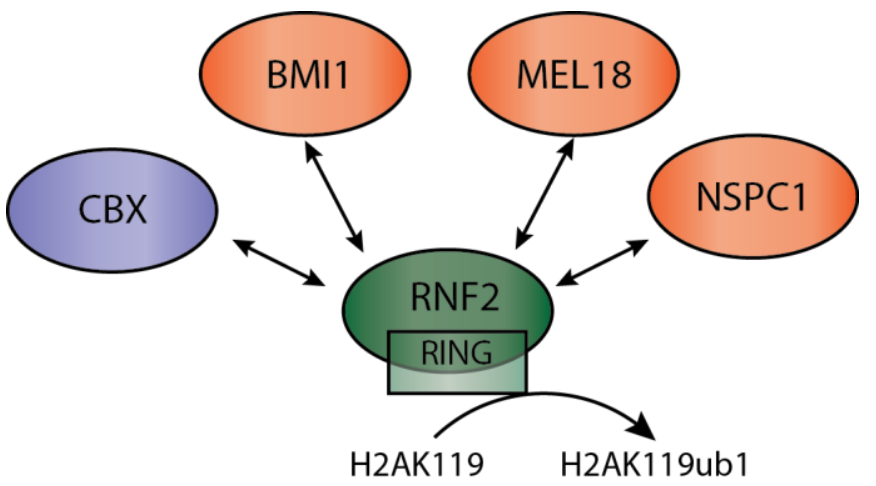

Fig.Il.2.2 Polycomb repressor complex 1.

The catalytic subunit RNF2 has E3 ubiquitin ligase activity. Interaction with RING proteins BMI1, MEL18 or NSPC1 as well as the chromodomain containing $\mathrm{CBX}$ proteins (CBX2, CBX4, CBX6, CBX7, and CBX8) diversify target gene specificity.

\section{II.2.3 Polycomb proteins and MDM2}

As an ubiquitin ligase, MDM2 not only transfers ubiquitin onto p53 and other proteins for their proteasomal degradation, but it can also transfer moieties to histones to regulate chromatin compaction. A study by Minsky and Oren in 2004 showed that MDM2 can physically interact with histones and transfer ubiquitin onto $\mathrm{H} 2 \mathrm{~A}$ in vitro and onto $\mathrm{H} 2 \mathrm{~B}$ both, in vitro and in vivo. As a result, transcription is repressed in a p53-independent and MDM2-RING-dependent mechanism (Minsky and Oren, 2004).

Since then, direct interactions of MDM2 with catalytic components of the PRC1 (RNF2) and PRC2 (EZH2) have been demonstrated (Wen et al., 2014; Wienken et al., 2016). In addition, MDM2 not only binds to the complexes but enhances their activity for trimethylation of H3K27 and monoubiquitination of H2AK119 of target genes. Such effects were seen in both mouse embryonic fibroblasts as an example for development as well as in a human colon carcinoma cell line (Wienken et al., 2016). As a result, MDM2 shares a regulatory profile with $\mathrm{PRC}$ complexes in the maintenance of stemness and repression of proliferation. 


\section{II.3 Genome integrity}

The cellular genome is challenged by a variety of external and internal factors causing DNA damage and mutations on a daily basis. The genetic material within a cell needs to be maintained without any damage and replicated exactly once per cell cycle to allow controlled proliferation and growth of tissues and organisms. Errors in replication result in mutations that can impair the functionality and cell cycle progression of the cell affected. Surveillance mechanisms are in place to monitor alterations in DNA structure and to activate signalling cascades initiating cell cycle arrest, DNA repair, as well as apoptosis. When mutations hit regulatory factors and render them inactive, the affected cell proliferates extensively. Uncontrolled proliferation not only harms surrounding tissues but also provides a basis for accumulating more mutations and tumourigenesis.

DNA, the molecular carrier of genetic information, consists of four nucleotides formed from nitrogen-containing bases cytosine, guanine, adenine, and thymine attached to a deoxyribose sugar moiety and a phosphate group. Covalent bonds between nucleotides in a chain as well as hydrogen bonds between two chains give rise to the characteristic double helix structure of DNA. DNA damage, characterised amongst others by alterations in the chemical structure of DNA bases, causes changes in the structure and poses problems for transcription and replication machineries using this stretch of DNA as a template. Natural (or endogenous) sources of damage arise from a variety of metabolic processes and include reactive oxygen and nitrogen species (ROS and NOS), reactive carbonyl species, as well as products of lipid peroxidation. An accumulation of ROS in a cell leads to oxidation of DNA bases, most frequently 8-oxoguanine, which causes mispairing of bases and thus leads to mutagenesis. The repair of these alterations with specialised enzymes like MTH1 (for 8-oxo-GTP) produce single stranded break (SSBs) in the cell, further contributing to genomic instability. Both altered bases as well as breaks in the DNA template are obstacles to DNA replication (De Bont, 2004).

In addition to endogenous sources of damage, a cell is also exposed to a plethora of exogenous damage sources, among which are ionising and ultraviolet irradiation as well as genotoxic compounds. The resulting double stranded breaks (DSBs) and misincorporation of bases lead to problems with DNA replication, rendering them vulnerable in S-phase (Dobbelstein and Sørensen, 2015). 
Cells sense and repair DNA lesions by a number of pathways collectively known as the DNA damage response (DDR; Fig.II.3.1). Common to all pathways involved, is the order of events taking place. First, a sensor - one or multiple proteins - identifies the lesion and transduces the signal via several mediators. Signal transduction within the signalling cascade is often mediated via post-translational modifications, e.g. phosphorylations by kinases. Eventually, an effector protein triggers a cellular response in the form of cell cycle arrest, DNA repair, chromatin remodelling, and apoptosis (Zhou and Elledge, 2000). Cells with defects in their DDR due to mutations are much more susceptible to DNA damage and accumulate unrepaired lesions over time.

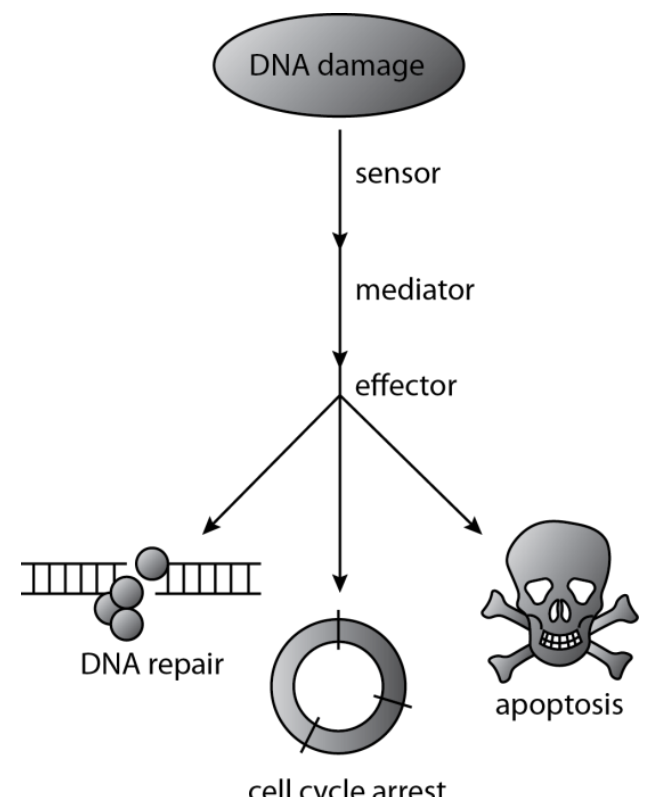

cell cycle arrest
Fig.II.3.1 The DNA damage response (DDR) - a signalling cascade.

As in many other types of signalling cascades, the DNA damage response involves a hierarchical signal transduction by different types of proteins. Sensors detect the damage at the lesion site and signal to mediators that in turn activate further mediators and eventually effectors. The signal is transduced by a number of post-translational modifications (most commonly phosphorylation) on substrates. This array of signalling allows a sustained response to damage by amplifying the signals until it is resolved. 


\section{II.3.1 DNA replication}

Eukaryotic replication initiates from thousands of replication origins interspersed throughout the genome to allow the replication of long linear chromosomes (Méchali, 2010). Tight spatial and temporal regulation of replication in a two-step fashion ensures that the genome of a cell is replicated exactly once per cell cycle - no less and no more.

The first step of replication occurs in G1 phase of the cell cycle, where replication origins are "licensed". For this, a pre-replication complex (pre-RC) is assembled at the origin sites (Fig.II.3.2; Méndez \& Stillman 2000). The complex itself consists of six origin recognition (ORC) proteins, Cdc6, Cdt1, as well as the hexameric helicase MCM2-7. It is assembled onto the origin in an ATP-dependent manner (Masai et al., 2010). Genome wide studies have identified a number of characteristics of metazoan origins including a specific origin G-rich repeat (OGRE) motif that is present in most origins and recognised by ORC proteins (Cayrou et al., 2011, 2012).

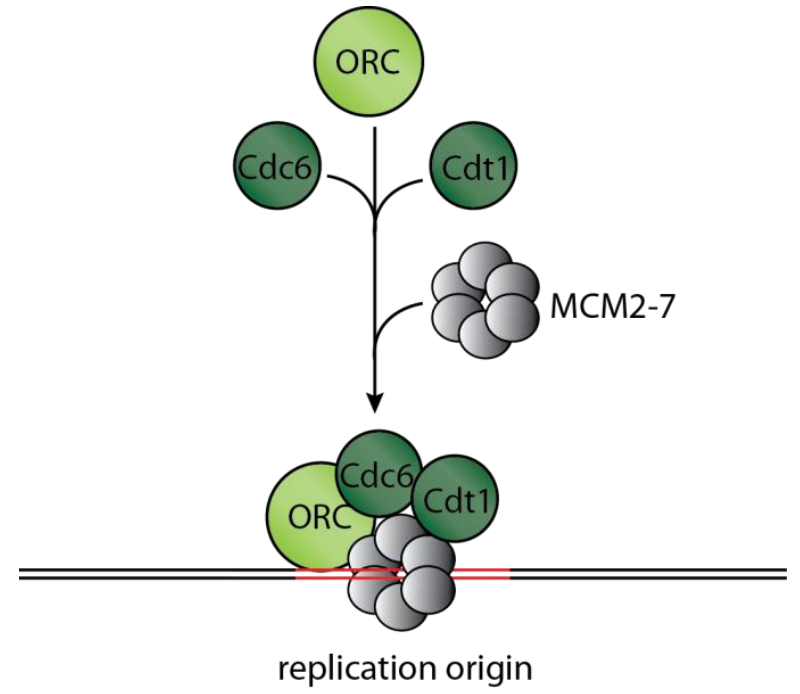

Fig.ll.3.2 Formation of the pre-replication complex.

Origin recognition (ORC) proteins recruit Cdc6 and Cdt1 to the replication origin that can load the MCM2-7 helicase onto the DNA with energy from ATP-hydrolysis to form a pre-replication complex (pre-RC).

In S-phase, a coordinated CDK response involving Cdc7 and CDK2 as well as other kinases and phosphatases (not discussed here) activates a subset of replication origins by phosphorylation of the MCM helicase (Montagnoli et al., 2006). Even though all potential origins are licensed, only about $10 \%$ of licensed origins are activated ("fired") under unperturbed replication conditions, whereas the remaining $90 \%$ serve as backup mechanisms for replicative stress conditions (Branzei and Foiani, 2005; Ge et al., 2007; McIntosh and Blow, 2012; Woodward et al., 2006). 
Active replisomes move forward bi-directionally from the origins of replication. The MCM helicase acts to unwind the double strand using the energy from ATP hydrolysis and releases stretches of single stranded DNA (SSDNA) that are coated with replication protein A (RPA) immediately (Fig.ll.3.3). DNA polymerases specific for leading (Pol $\varepsilon$ ) and lagging strand (Pol $\delta$ ) use ssDNA stretches as templates for synthesis of new strands. Replication continues until two forks converge and terminate their activity by releasing the DNA template.

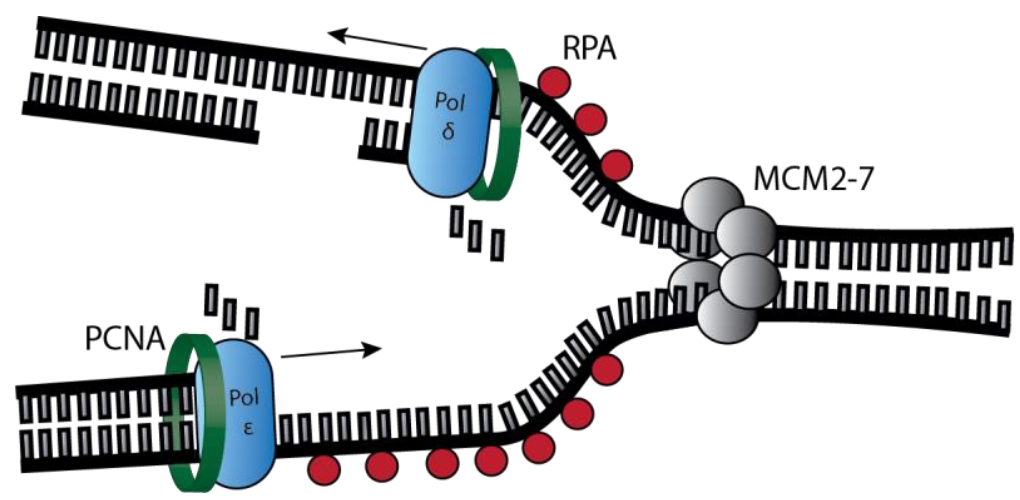

Fig.II.3.3 Schematic diagram of a replication fork (simplified).

A replication fork consists of two antiparallel DNA strands (leading and lagging strand) opened up by the MCM2-7 helicase. Stretches of SsDNA exposed by the unwinding of the helix are coated by RPA to protect from degradation. DNA polymerases ( $\varepsilon$ for leading strand and $\delta$ for the lagging strand) are loaded onto and kept in proximity to the template DNA by the PCNA clamp loading complex for DNA synthesis.

\section{II.3.2 Replicative stress}

Replication forks are very vulnerable to impediments they encounter while sliding along their DNA template. These obstructions can occur in the form of DNA damage at the level of single bases, breaks in one or both strands, secondary structures in the template, as well as proteins that are bound to the DNA preventing the progression of the replication fork. Stalled replication forks stop at the lesion or obstacle but are able to resume replication when it is repaired or removed. Collapsed replication forks have lost the ability to resume DNA synthesis as the core replication machinery dissociates from the template at the lesion (Petermann and Helleday, 2010). 
Downstream of a stalled replication fork, the MCM helicase continues to unwind the double strand for a few hundred bases. Single stranded DNA exposed by this separation of the two strands extends the short SsDNA stretches that occur during replication and serves as signal for replicative stress. RPA coats SSDNA and interacts with the main

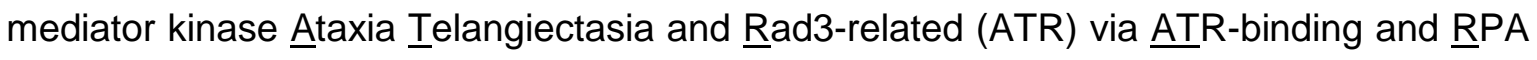
Interacting Protein (ATRIP; Byun et al. 2005). Upon activation, ATR phosphorylates hundreds of substrates at a Ser/Thr-Glu motif to activate further mediators and effectors in the damage signalling cascade (Matsuoka et al., 2007). Common readouts for replicative stress are proteins downstream of the ATR response inlcuding checkpoint protein 1 (CHK1), RPA, or the histone variant $\mathrm{H} 2 \mathrm{AX}(\mathrm{yH} 2 \mathrm{AX}$; $\mathrm{pH} 2 \mathrm{AX}-\mathrm{Ser} 139)$ as well as an accumulation of stalled replication forks, firing replication origins, and persistent ssDNA (Dobbelstein and Sørensen, 2015; Maréchal and Zou, 2013).

In order to prevent genomic instability, an intra-S-phase checkpoint delays the progression of S-phase in response to replicative stress (Fig.ll.3.4). The checkpoint works in two pathways downstream of the main damage mediator kinases ATR and Ataxia Telangiectasia Mutated (ATM; Tasat \& Yakisich 2010).

In response to DSBs, ATM is recruited to sites of damage by the sensor complex MRN and its kinase activity is enhanced in response (Lee and Paull, 2005). Substrates such as CHK2, BRCA1, and p53 are phosphorylated by ATM to mediate effects on repair, cell cycle arrest, and apoptosis (Lavin, 2008; Shiloh, 2003). ATR on the other hand is activated by single stranded breaks or in response to replicative stress in form of excess ssDNA. This leads to phosphorylation of CHK1 at serine residues 317 and 345 as well as ATM at serine 1981 (Stiff et al., 2006; Zhao and Piwnica-Worms, 2001). 


\section{Fig.Il.3.4 The intra-S checkpoint.}

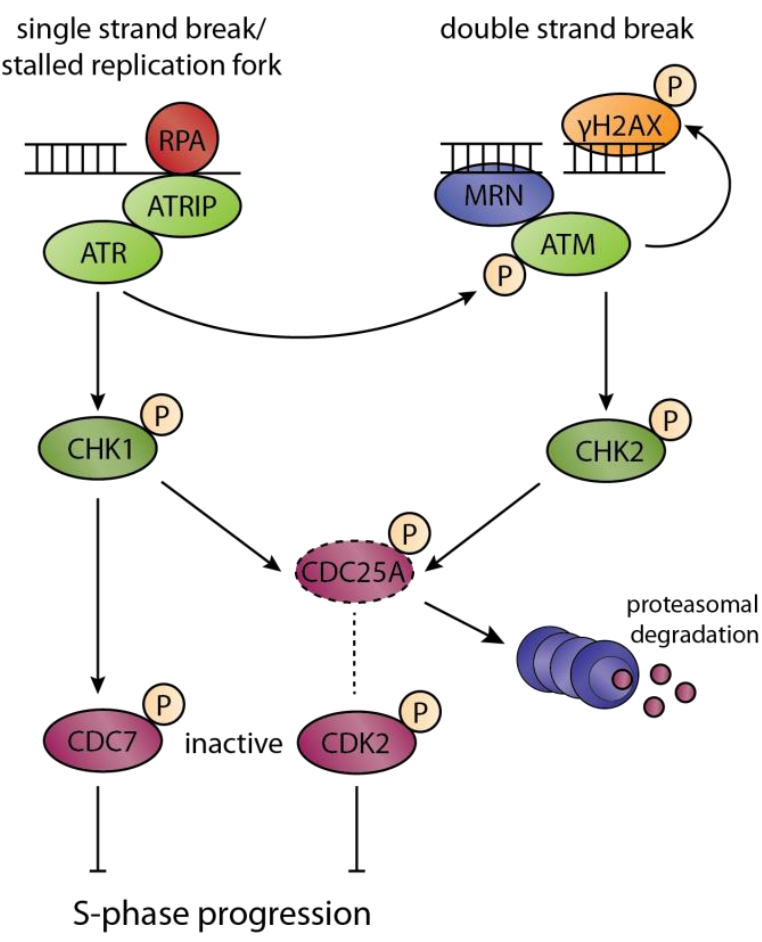

Double strand breaks bound by the MRN complex recruit and activate ATM kinase. Single stranded DNA arising from stalled replication forks or single stranded breaks are bound by ATR via ATRIP and activate kinase activity. Both ATM and ATR, phosphorylate a large range of substrates including the mediator kinases $\mathrm{CHK} 1$ and CHK2. Crosstalk between the two kinases as well as ATR and ATM exists and promotes a sustained response to DNA damage until the lesion is resolved. The phosphorylation of the CHK1/2 substrate CDC25A leads to its proteasomal degradation. The loss of CDC25A activity fails to activate CDK2, which normally induces CDC45-mediated DNA polymerase recruitment. Additionally, CHK1 also phosphorylates and inhibits $\mathrm{CDC7}$, thus S-phase progression is halted in two ways.

Both pathways are heavily interlinked and converge on the mediator kinases of the second wave: CHK1 and CHK2. These kinases are responsible for the phosphorylation of CDC25A causing it to be degraded by the proteasome (Mailand et al., 2000). CDC25A is a phosphatase which removes an inhibitory phosphate group from CDK2, a kinase essential for CDC45-dependent recruitment of DNA polymerases for origin firing (Falck et al., 2002; Owens et al., 1997; Takisawa et al., 2000). In addition, CHK1 phosphorylates, and by that inhibits, $\mathrm{CDC7}$, a kinase that phosphorylates the pre-RC complex for recruiting further factors in conjunction with CDK2 (Petermann et al., 2010). As a result, activation of ATR and ATM kinases by DNA damage and replicative stress cause a signalling cascade that acts as a checkpoint in S-phase.

\section{II.3.3 Replicative stress in cancer}

Genome instability is now considered as one of the hallmarks of cancer but whether it is cause or consequence of tumourigenesis has long been under debate (Hanahan and Weinberg, 2011; Tomlinson and Bodmer, 1999). More recent evidence suggests that DNA damage arises as a result of replicative stress early on in transformation but can be kept under control by an intact DDR (Bartkova et al., 2005; Gorgoulis et al., 2005).

Many oncogenes drive uncontrolled proliferation and induce replicative stress as a result. The activation of checkpoints and damage signalling increase selective pressure on the 
system to mutate important mediators of the DDR to provide further proliferative advantages. Subsequently, cells lacking checkpoints no longer arrest their cell cycle for repair or die from apoptosis but rather accumulate even more damage by replicative stress and drive tumourigenesis forward (Bartkova et al., 2006; Dobbelstein and Sørensen, 2015; Halazonetis et al., 2008). Most drugs that are currently used in the clinics to treat cancers, damage DNA and cause replicative stress either directly or indirectly (Dobbelstein and Sørensen, 2015). Examples of direct interference with replication are outlined below.

Direct modifications of DNA bases by platinum compounds (e.g. carboplatin, cisplatin) or alkylating agents (e.g. mitomycin C, cyclophosphamide) cause crosslinking between bases or DNA strands making replication of the affected stretches difficult (Fu et al., 2012; Henry-Mowatt et al., 2003; Wang and Lippard, 2005). Intra-strand crosslinks prevent accurate base pairing and their replication requires the recruitment of specialised translesion polymerases to the site. Polymerases of this kind are more flexible to accept incorrect base pairing. However, these polymerases catalyse these reactions in a less processive and more error prone way as they lack a proofreading activity (Lehmann et al., 2007). Inter-strand crosslinks (ICL) on the other hand prevent the dissolution of the two strands in the double helical structure and thus the accessibility of the template DNA (Deans and West, 2011).

Antimetabolites are another class of chemotherapeutic compounds used to induce replicative stress in cancer cells. Structural analogues of DNA bases are incorporated during DNA synthesis but block further elongation and cause replication fork stalling. The nucleoside analogue 2'-2'-difluoro-deoxycytidine (Gemcitabine) additionally inhibits ribonucleotide reductase (RNR), an enzyme important for maintaining dNTP pools. Other drugs like hydroxyurea and 5-fluorouracil (5-FU) have similar mechanisms of action inhibiting nucleoside synthesis by targeting RNR and thymidylate synthetase, respectively (Ewald et al., 2008).

Topoisomerase inhibitors, including camptothecin and etoposide, present another class of chemotherapy drugs. Topoisomerases relax DNA supercoiling that arises around the replication fork due to unwinding of the double helix by breaking and re-ligating DNA strands. Inhibitors bind to these enzymes and the resulting complexes form a physical barrier to replication triggering a repair response (Pommier, 2013). Efforts of removing the complexes often result in DSB formation which further adds to genomic instability (Regairaz et al., 2011). 


\section{III.3.4 Replication and transcription - similarities and differences}

In many physiological settings, a crosstalk between two processes occurring simultaneously provides advantages, as seen e.g. with transcription-coupled repair. However, there can also be negative consequences when two processes occur at the same time and genomic location. This is the case for transcription and replication, both of which are processes that require access to the DNA strands as templates. Both processes involve a specialised polymerase for the synthesis of a complementary strand and DNA-RNA intermediate structures.

RNA polymerase that is responsible for transcription binds to dsDNA and opens up the helix within its active site. The synthesis of a complementary RNA strand results in a highly dynamic short stretch of DNA:RNA hybrid structures of about 10 nucleotides in length. As the RNA chain elongates, it loses its association with the DNA, is bound by RNA-binding proteins and exits the polymerase via a separate channel as its DNA complement to avoid the persistence of DNA:RNA hybrids (Alberts et al., 2007; Stryer et al., 2002; Westover, 2004).

DNA polymerase, the enzyme catalysing DNA synthesis, consists of two polymerase subunits ( $\delta$ and $\varepsilon$ in mammalian cells) that synthesise complements to ssDNA templates that have been unwound by the MCM helicase. At the leading strand, one long complementary strand of DNA is synthesised. At the lagging strand on the other side, only short fragments of DNA (called Okazaki fragments) are synthesised as the polymerase needs to wait for the helicase to unwind the strands further to initiate the next segment to be replicated. This initiation of replication is mediated by the synthesis of short complementary RNA stretches by DNA primase that serve as primers for the lagging strand polymerase $\varepsilon$. These DNA-RNA hybrids are very short (about 10 nucleotides) and have a short half-life as they are rapidly degraded by RNase H (Alberts et al., 2007; Stryer et al., 2002). 


\section{III.3.5 Replication-transcription conflicts - when separation goes wrong}

Both transcription and replication are dependent on the template sequence of DNA molecules and direct access in order to synthesise complementary strands. How these two processes can co-exist and how conflicts between them are regulated has been a longstanding question and is solved differently in different organisms.

In mammalian cells, transcription and replication are spatially and temporally separated in S-phase cells to prevent encounters of the two multiprotein complexes sliding along the DNA (Smirnov et al., 2014). Detailed analysis of nascent transcripts showed that regions transcribed in early S-phase are replicated in late S-phase and vice versa (MeryetFiguiere et al., 2014). A dysregulation of this pattern leads to conflicts between both processes and is a source of genomic instability.

As mammalian transcription and replication is bi-directional, collisions of replisomes with transcription complexes can occur co-directionally or "head-on", the latter of which is more detrimental (Merrikh et al., 2011; Srivatsan et al., 2010). In both scenarios, the replication fork stalls as it cannot progress past the transcription complex. In co-directional collisions, the replication fork is able to restart after transcription is terminated, whereas head-on collisions result in transcription-associated recombination (TAR) accompanied by a collapse of the replication fork (Prado and Aguilera, 2005).

Even though the term "collision" implies a physical contact of the two polymerase complexes, it is not entirely known whether this is the case or whether the effects observed are solely due to changes in the chromatin environment of the approaching complexes (García-Muse and Aguilera, 2016). Torsional stress generated by positive and negative supercoiling around the RNA polymerase is normally relieved by topoisomerases of type I or II, catalysing single or double strand breaks, respectively. Inefficient resolution of negative supercoiling can result in DNA melting and by that a change in the helical structure of the DNA to a non-B form (García-Muse and Aguilera, 2016; Pannunzio and Lieber, 2016). An example of a structure that is formed as a result of transcriptional supercoiling is G-quadruplexes. These structures consist of a repeat of guanine resides that form four-strand interactions posing as obstacles to replication (Kim and JinksRobertson, 2011; Yadav et al., 2014).

Another type of non-B structure that develops due to local DNA melting are so-called Rloops. Unwound DNA double strands facilitate the re-annealing of complementary RNA molecules that exit RNA polymerase as a nascent transcript in the absence of 
ribonucleoprotein particles (Huertas and Aguilera, 2003). The consequence is a DNARNA hybrid structure with a displaced ssDNA strand (Fig.II.3.5).

Hybrids between DNA and RNA are thermodynamically more stable than dsDNA and adopt a structural intermediate between $A$ and $B$ form dsDNA that impairs replication (Roberts and Crothers, 1992; Shaw and Arya, 2008). Their special conformation serves as a recognition element for specialised enzymes that remove R-loops in energyconsuming reactions. Among these enzymes is RNase $\mathrm{H} 1$, an endonuclease that cleaves the RNA moiety of the R-loop (Wahba et al., 2011).

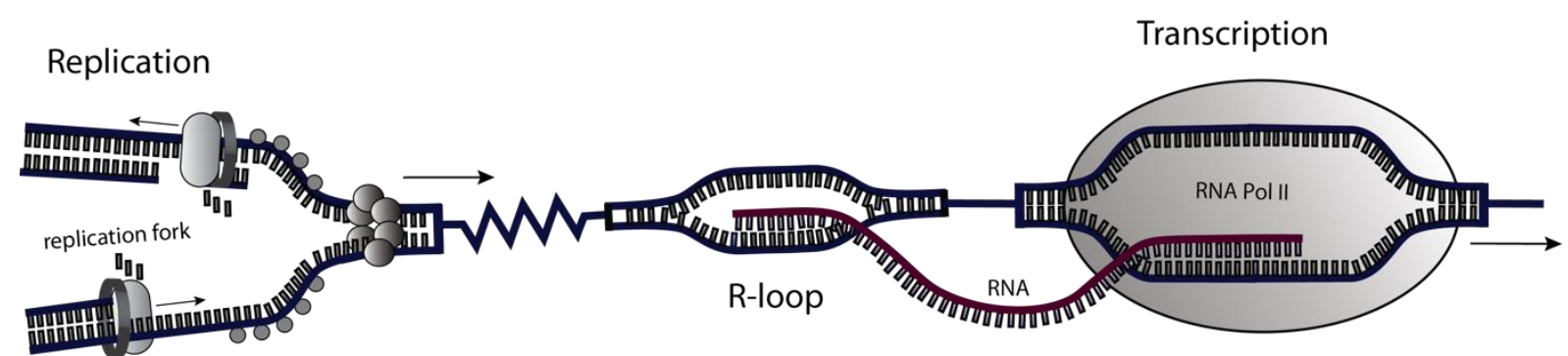

Fig.II.3.5 Thread-back model of R-loop formation.

Co-transcriptional R-loops form when the nascent mRNA is incompletely coated by ribonucleoproteins and inserts back into a complementary DNA duplex. The resulting hybrid of DNA and RNA is a very stable structure and stops replication fork progression. 


\section{II.4 Project Aim}

The aim of this thesis is to determine a role of p53 in protecting a cell's genome from damage during DNA replication. Preliminary data from my Master's thesis suggested that p53 has a supportive role in DNA replication. This thesis expands on these initial findings by studying the progression of single replication forks in a number of cell systems in the absence and presence of the tumour suppressor p53.

The osteosarcoma cell line U2OS is used as an example for transformed cells with high intrinsic replicative stress conditions and wildtype p53 expression. In addition, we also make use of primary thymocytes isolated from mice carrying p53 knockout alleles and their wildtype littermates. A third system to study the effect of p53 depletion are mouse embryonic fibroblasts from genetically modified embryos carrying floxed p53 alleles. Crerecombination in vitro allows us to study short-term effects of p53 depletion in primary cells. Using these cell systems, we will try to understand whether p53 protects cells from endogenous DNA damage during replication and whether it does so by acting as a transcription factor in S-phase. DNA fiber assays used to analyse effects on single replication forks provide a readout in the form of the length of a replicated stretch of DNA in a given time. Differences in length can result not only from impaired replisomes that progress along the template more slowly, but also from a less stable association of the replisome with its template and obstacles that cause it to stall.

Given that the mechanism by which p53 seems to support DNA replication is unknown, it is essential to distinguish between replication fork velocity and processivity of the DNA polymerase. The development of a "fork stalling fiber assay" allows us to analyse the two parameters separately and draw more accurate conclusions on how replication is affected.

As p53 is a transcription factor, we hypothesise that it is highly likely that p53 supports DNA replication through one of its target genes. Therefore we aim to identify a transcriptional target of p53 that is essential for supporting DNA replication and the mechanism by which it does so. MDM2, a transcriptional target of p53, has a noncanonical role in modifying chromatin compaction. As chromatin modifications can also affect replication fork progression, we will assess the role of MDM2 in DNA replication to find a mechanism by which p53 acts as a pro-active guardian of the genome. 


\section{Materials}

\section{III.1 Technical devices}

Device

Producer

Blotting chamber

Biozym

Cell counting chamber Neubauer improved

Bran

Centrifuge $5415 R$

Eppendorf

Centrifuge 5810R

Eppendorf

Chemiluminescence imager Chemidoc XRS+

Biorad

Chemiluminescence imager Chemocam HR 163200

Intas Science Imaging

Electrophoresis system for SDS-PAGE

Amersham Biosciences

Freezer $-20^{\circ} \mathrm{C}$

Liebherr

Freezer $-80^{\circ} \mathrm{C}$

Heraeus, Thermo Scientific

Heating Block Thermomixer comfort

Eppendorf

Ice machine B100

Ziegra

Laminar flow cabinet Hera safe

Heraeus, Thermo Scientific

Light microscope Axovert 40C

Zeiss

Magnetic Stirrer Variomag Mono

Komet

Microscope, Axio Scope.A1

Zeiss

Pathway HT Cell Imaging System

BD Biosciences

PCR machine for qPCR CFX96, C1000

BioRad

PCR machine Thermocycler T Personal

Biometra

$\mathrm{pH}$ meter inoLab

WTW GmbH

Pipet Aid Portable \#4XXX-200

Drummond

Pipets, Eppendorf Research

Eppendorf

Power Supply

Biometra

Refridgerator $4^{\circ} \mathrm{C}$

Liebherr

Roller RM5-30V

CAT

Scales Acculab ALC-6100.1

Sartorius

Scales LE623S

Sartorius

Scanner CanoScan 8600F

Canon

Sonication device Bioruptor

Diagenode

Spectrophotometer NanoDrop ND-1000

PeqLab

Thermomixer Comfort

Eppendorf

Timer

Oregon Scientific

Vortex Genie 2

Scientific Instruments 


\section{III.2 Consumables}

\section{Consumable}

96-well imaging plate

96-well plate for qPCR

Cell culture dishes $(10 \mathrm{~cm}, 15 \mathrm{~cm})$

Bacteria culture dish $(10 \mathrm{~cm})$

Cell culture plate (6-well, 12-well)

Cell culture plate 24-well

Cell scraper $(16 \mathrm{~cm}, 25 \mathrm{~cm})$

Coverslips

Cryo-tubes for cell freezing

Glass pipets $(5 \mathrm{ml}, 10 \mathrm{ml}, 25 \mathrm{ml})$

Glass Slides Superfrost

Parafilm

Pipet tips (10 $\mu \mathrm{L}, 20-200 \mu \mathrm{L}, 1,000 \mu \mathrm{L}$ )

Protran nitrocellulose transfer membrane

Reaction tube $(0.5 \mathrm{~mL}, 1.5 \mathrm{~mL}, 2.0 \mathrm{~mL})$

Reaction tube $(15 \mathrm{~mL}, 50 \mathrm{~mL})$

Safe-lock reaction tube $(1.5 \mathrm{~mL})$

Sterile filter $(0.2 \mu \mathrm{M}$ and $0.45 \mu \mathrm{M})$

Syringe canula

Whatman paper

\section{Producer}

Becton Dickinson

4titude

Greiner

Sarstedt

Greiner

Costar

Sarstedt

Menzel, Roth

Nunc

Sarstedt

Menzel

Sigma-Aldrich

Greiner

Whatman

Eppendorf

Greiner

Eppendorf

Millipore

B.Braun

Whatman 


\section{III.3 Chemicals and reagents}

\section{Chemical}

Acetic acid

Agar

Agarose

Albumin Fraction V (Bovine Serum Albumine, BSA)

Ammonium persulfate (APS)

Ampicillin

Chlorodeoxyuridine (CldU)

Chloroform

Deoxynucleotide triphosphates (dNTPs)

Dimethyl sulfoxide (DMSO)

DNA ladder GeneRuler

EdU (5-ethynyl-2'-deoxyuridine)

Ethanol $99.8 \%$

Formaldehyde, $37 \%$ solution

Glycerol >99\% p.a.

Glycine $>99 \%$ p.a.

Hydrogen chloride $(\mathrm{HCl})$

lododeoxyuridine (IdU)

Isopropanol

Lipofectamine 2000/3000

Methanol >99\% (MetOH)

Milk powder

MitoSOX red mitochondrial superoxide indicator

Nailpolish

Nuclease-free $\mathrm{H}_{2} \mathrm{O}$

PageRuler Prestained Protein Ladder

Paraformaldehyde (PFA) 37\%

Pefablock SC protease inhibitor

Pepstatin A

Ponceau S

Potassium Chloride

Potassium hydrogenphosphate $\left(\mathrm{KH}_{2} \mathrm{PO}_{4}\right)$

Random hexamer primers

\section{Producer}

Roth

Sigma-Aldrich

Roth

Roth

Roth

Roth

Sigma-Aldrich

Roth

Primetech

AppliChem

Fermentas

Thermo Fisher

Roth

Roth

Roth

Roth

Roth

Sigma-Aldrich

Roth

Invitrogen

Roth

Roth

Thermo Fisher

essence

Ambion

Fermentas

Sigma-Aldrich

Roth

AppliChem

Roth

AppliChem

Roth

Thermo Scientific 
Rotiphorese Gel 30

Roth

Sodium chloride $(\mathrm{NaCl})$

Roth

Sodium deoxycholate

AppliChem

Sodium dodecyl sulfate (SDS)

Roth

Sodium dodecyl sulphate (SDS)

BioRad

Sodium ethylene diamine tetra-acetic acid (Na-EDTA)

Roth

Sodium hydrogenphosphate monohydrate $\left(\mathrm{NaHPO}_{4} \times \mathrm{H}_{2} \mathrm{O}\right)$ Roth

Sodium hydroxide $(\mathrm{NaOH})$

Sigma-Aldrich

Sodium(di-)hydrogenphosphatedihydrate $\left(\mathrm{Na}_{2} \mathrm{HPO}_{4}\right) \times 2 \mathrm{H}_{2} \mathrm{O}$ Roth

SYBR green

Invitrogen

Tetramethylethylenediamine (TEMED)

Merck

Trehalose dehydrate

Usb Corp.

Trisamine (Tris) Pufferan $>99 \%$ p.a.

Roth

Triton-X100

AppliChem

TRIZOL

Invitrogen

Tween 20

Applichem

Vectashield mounting medium

Vector Laboratories 
Cell lysis buffer

\begin{tabular}{ll}
\hline urea & $2.5 \mathrm{M}$ \\
RIPA lysis buffer & $60 \%$ \\
Pefa & $1 \%$ \\
PA & $1 \%$ \\
L/A & $0.1 \%$
\end{tabular}

Fibre Assay Blocking Solution

\begin{tabular}{ll}
\hline BSA & $3 \%$ \\
Tween20 & $0.1 \%$
\end{tabular}

in PBS pH 7.4
Ponceau S

\begin{tabular}{ll}
\hline Ponceau S & $0.5 \%$ \\
acetic acid & $1 \%$ \\
in $\mathrm{dd}_{2} \mathrm{O}$ &
\end{tabular}

RIPA Lysis Buffer

\begin{tabular}{ll}
\hline Triton X-100 & $1 \%$ \\
Na-deoxycholate & $1 \%$ \\
$\mathrm{NaCl}$ & $150 \mathrm{mM}$ \\
EDTA & $10 \mathrm{mM}$ \\
Tris, $\mathrm{pH} 7.5$ & $20 \mathrm{mM}$ \\
in $\mathrm{ddH}_{2} \mathrm{O}$ &
\end{tabular}

Fibre Assay Fixative

\begin{tabular}{ll}
\hline MetOH & $75 \%$ \\
Acetic Acid & $25 \%$
\end{tabular}

Fibre Assay Spreading Buffer

\begin{tabular}{|c|c|}
\hline $\begin{array}{l}\text { Tris pH } 7.4 \\
\text { EDTA } \\
\text { SDS } \\
\text { in } \mathrm{ddH}_{2} \mathrm{O}\end{array}$ & $\begin{array}{l}200 \mathrm{mM} \\
50 \mathrm{mM} \\
0.5 \%\end{array}$ \\
\hline 6x Laemmli Buffer & \\
\hline $\begin{array}{l}\text { Tris pH } 6.8 \\
\text { glycerine } \\
\text { SDS } \\
\text { dithiotreitol } \\
\text { bromophenol blue } \\
\text { in } \text { ddH }_{2} \mathrm{O}\end{array}$ & $\begin{array}{l}0.35 \mathrm{M} \\
30 \% \\
10 \% \\
9.3 \% \\
0.02 \%\end{array}$ \\
\hline
\end{tabular}

Phosphate Buffered Saline (PBS)

$\begin{array}{ll}\mathrm{NaCl} & 24 \mathrm{mM} \\ \mathrm{KCl} & 0.27 \mathrm{mM} \\ \mathrm{Na}_{2} \mathrm{HPO}_{4} \times 7 \mathrm{H}_{2} \mathrm{O} & 0.81 \mathrm{mM} \\ \mathrm{KH}_{2} \mathrm{PO}_{4} & 0.15 \mathrm{mM}\end{array}$

in $\mathrm{ddH}_{2} \mathrm{O}$
SDS-PAGE Running Buffer

$\begin{array}{ll}\text { Tris } & 25 \mathrm{mM} \\ \text { glycin } & 86.1 \mathrm{mM} \\ \mathrm{SDS} & 3.5 \mathrm{mM} \\ \text { in } \mathrm{ddH}_{2} \mathrm{O} & \end{array}$

Tris Buffered Saline (TBS) + Tween20 (TBS-T)

\begin{tabular}{ll}
\hline Tris & $50 \mathrm{mM}$ \\
$\mathrm{NaCl}$ & $150 \mathrm{mM}$ \\
Tween20 & $0.1 \%$ \\
in $\mathrm{ddH}_{2} \mathrm{O}$ &
\end{tabular}

Western Blot Transfer Buffer

$\begin{array}{ll}\text { Tris } & 25 \mathrm{mM} \\ \text { glycin } & 192 \mathrm{mM} \\ \text { MetOH } & 20 \% \\ \text { in } \text { dd }_{2} \mathrm{O} & \end{array}$

FACS binding buffer

\begin{tabular}{lr}
\hline Hanks balanced solution + \\
$\mathrm{CaCl}_{2}$ & $5 \mathrm{mM}$ \\
$\mathrm{MgCl}_{2}$ & $5 \mathrm{mM}$ \\
$\mathrm{BSA}$ & $1 \%$
\end{tabular}


III.5 Enzymes and reaction buffers

$\begin{array}{lll}\text { Enzyme } & \text { Buffer } & \text { Producer }\end{array}$

New England Biolabs

M-MuLV reverse transcriptase $\quad 10 x$ MuLV buffer $\quad$ (NEB)

Taq DNA Polymerase for qPCR 10x Taq buffer $(+\mathrm{KCl}$,

$-\mathrm{MgCl}_{2}$ ) Primetech, Fermentas

III.6 Commercial Kits

Name

Producer

Click-iT EdU Alexa488 HCS assay kit

Thermo Fisher

Immobilon Western HRP Substrate Peroxide Solution

Millipore, Merck

Pierce BCA Protein assay kit

Thermo Fisher

PureYield Plasmid Midiprep System

Promega

SuperSignal Western Femto Maximum Sensitivity Substrate

Thermo Fisher

TruSeq RNA LT SamplePrep Kit

Illumina 
III.7 Chemotherapeutics and Pharmacological inhibitors

\begin{tabular}{lll} 
Name & Target & Producer \\
\hline $\begin{array}{l}\text { DRB (5,6-Dichlorobenzimidazole 1- } 3 \text {-D- } \\
\text { ribofuranoside) }\end{array}$ & CDK9 & Sigma \\
DZNep & EZH2 & Cayman Chemicals, \\
EPZ6438 & EZH2 & Selleckchem \\
Gemcitabine (2',2'-difluorodeoxycytidine & & Eli Lilly \\
(dFdC)) & & Sigma \\
Hydroxyurea & RNR & Selleckchem \\
LDC067 & CDK9 & \\
Nutlin-3a & & Sigma Aldrich \\
PHA-766491 & Mdm2 & \\
PTC-209 & CDK7 & Selleckchem
\end{tabular}




\section{III.8 Oligonucleotides}

\begin{tabular}{|c|c|c|c|}
\hline Name & SiRNA ID & Target & Sequence \\
\hline ctrl\#1 \& 2 & neg. control\#1 \& 2 & & undisclosed \\
\hline \multirow{2}{*}{ EED\#1 } & \multirow{2}{*}{ s16624 } & \multirow{2}{*}{ EED } & GCUUUACGAUUAUGGAAUAtt \\
\hline & & & UAUUCCAUAAUCGUAAAGCat \\
\hline \multirow{2}{*}{ EED\#2 } & \multirow{2}{*}{ s16625 } & \multirow{2}{*}{ EED } & GGCAUAAUUAGGAUAAUAAtt \\
\hline & & & UUAUUAUCCUAAUUAUGCCtC \\
\hline \multirow{2}{*}{ EED\#3 } & \multirow{2}{*}{ s16626 } & \multirow{2}{*}{ EED } & CAUUAGUGUUUGCAACUGUtt \\
\hline & & & ACAGUUGCAAACACUAAUGga \\
\hline \multirow{2}{*}{$\mathrm{EZH} 2 \# 1$} & \multirow{2}{*}{ s4916 } & \multirow{2}{*}{$\mathrm{EZH} 2$} & GCUGACCAUUGGGACAGUtt \\
\hline & & & UACUGUCCCAAUGGUCAGCgg \\
\hline \multirow{2}{*}{ EZH2\#1 } & \multirow{2}{*}{ s4917 } & \multirow{2}{*}{$\mathrm{EZH} 2$} & GUGUAUGAGUUUAGAGUCAtt \\
\hline & & & UGACUCUAAACUCAUACACct \\
\hline \multirow{2}{*}{ EZH2\#1 } & \multirow{2}{*}{ s4918 } & \multirow{2}{*}{$\mathrm{EZH} 2$} & GGCACUUACUAUGACAAUUtt \\
\hline & & & AAUUGUCAUAGUAAGUGCCaa \\
\hline \multirow{2}{*}{ Mdm2\#1 } & \multirow{2}{*}{ custom select } & \multirow{2}{*}{ MDM2 } & GCCAUUGCUUUUGAAGUUAtt \\
\hline & & & UAACUUCAAAAGCAAUGGCtt \\
\hline \multirow{2}{*}{ Mdm2\#2 } & \multirow{2}{*}{ s224037 } & \multirow{2}{*}{ MDM2 } & AGACCCUGGUUAGACCAAAtt \\
\hline & & & UUUGGUCUAACCAGGGUCUct \\
\hline \multirow{2}{*}{ Mdm2\#3 } & \multirow{2}{*}{ s8629 } & \multirow{2}{*}{ MDM2 } & AGUCUGUUGGUGCACAAAAtt \\
\hline & & & UUUUGUGCACCAACAGACUtt \\
\hline \multirow{2}{*}{ p53\#1 } & \multirow{2}{*}{ s605 } & \multirow{2}{*}{ p53 } & GUAAUCUACUGGGACGGAAtt \\
\hline & & & UUCCGUCCCAGUAGAUUACca \\
\hline 553\#? & 5607 & $n 53$ & GGUGAACCUUAGUACCUAAtt \\
\hline p0ง+t< & Sout & p50 & UUAGGUACUAAGGUUCACCaa \\
\hline RNF?\#1 & 12067 & RNF2 & GGCUAGAGCUUGAUAAUAAtt \\
\hline nIVr L\# I & 512001 & RIVre & UUAUUAUCAAGCUCUAGCCca \\
\hline RNF?\#? & s12068 & RNF? & CAAACGGACCAAAACAUCUtt \\
\hline nIVICHL & $51 \angle 000$ & nivic & AGAUGUUUUGGUCCGUUUGtt \\
\hline RNF2\#3 & s12069 & RNF? & GGAGUGUUUACAUCGUUUUtt \\
\hline nIVז<\#s & 512005 & nivic & AAAACGAUGUAAACACUCCtt \\
\hline SII712\#1 & \$3967 & SII712 & GGAUGUAAGUUGUCCAAUAtt \\
\hline SULICका & 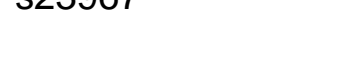 & (6) & UAUUGGACAACUUACAUCCtt \\
\hline su712\#2 & s23968 & SU712 & GGACCUACGUUGCAGUUCAtt \\
\hline & & & UGAACUGCAACGUAGGUCCct \\
\hline
\end{tabular}




\section{III.9 Plasmids}

\begin{tabular}{lll} 
Name & Publication & Source \\
\hline pcDNA3, pLenti-GFP & Oliner et al. 1992 & Invitrogen \\
pCMV-MDM2 wt & Boyd et al. 2000 & Addgene \# 16441 \\
pCMV-MDM2 C464A & Scott et al. 2011 & Addgene \#31216 \\
pLenti6-V5-DEST-RNF2 & Britton et al. 2014 & Addgene \#60365 \\
pICE-RNaseHI-WT-NLS- & & \\
$\begin{array}{l}\text { mCherry } \\
\text { pICE-RNaseHI-D10R-E48R- }\end{array}$ & Britton et al. 2014 & Addgene \#60367 \\
NLS-mCherry & &
\end{tabular}

\section{III.10 Antibodies}

III.10.1 Antibodies for Western Blot

\begin{tabular}{lllll} 
Antibody & Source & Dilution & Producer & Catalogue nr. \\
\hline Ezh2 (D2C9) XP & rabbit & $1: 1000$ & Cell Signalling & 5246 \\
H2AK119ub1 & rabbit & $1: 1000$ & Millipore & ABE569 \\
H2AX pS319 & rabbit & $1: 1000$ & Cell Signalling & 2577 \\
H3K27me3 & rabbit & $1: 1000$ & Diagenode & pAb-069-050 \\
HSC70 & mouse & $1: 15,000$ & Santa Cruz & sc-7298 \\
MDM2 & mouse & $1: 300$ & Calbiochem & OP\#46 \\
p21 & mouse & $1: 1000$ & Cell Signalling & 2947 \\
p53 (DO-1) & mouse & $1: 1000$ & Santa Cruz & sc126 \\
p53-CM5p & rabbit & $1: 200$ & Vector & n/a anymore \\
& & & Laboratories & \\
RING1B & rabbit & $1: 500$ & Cell Signalling & 5694 \\
SUZ12 & rabbit & $1: 1000$ & Cell Signalling & 3737 \\
$\beta$-actin & mouse & $1: 50,000$ & abcam & ab6276-100 \\
mCherry & mouse & $1: 20,0000$ & abcam & ab125096
\end{tabular}




\section{III.10.2 Antibodies for Fiber Assay}

\begin{tabular}{lllll} 
Antibody & Source & Dilution & Producer & Catalogue nr. \\
\hline BrdU/CldU & rat & $1: 500,1: 300$ & AbDSerotec & OBT0030 \\
BU1/75 & & $1: 1000$ & abcam & ab6326 \\
\hline BrdU/ldU B44 & mouse & $1: 500$ & Becton Dickinson & 347580 \\
\hline ssDNA & mouse & $1: 1000$ & Millipore & MAB3034
\end{tabular}

\section{III.10.3 Secondary Antibodies for Western Blot}

\section{Antibody}

Dilution Producer

Catalogue nr.

HRP-coupled AffiniPure

1:10,000 Jackson Immunoresearch

711-036-152

$F(a b ') 2$ fragment, anti

mouse $\lg G(\mathrm{H}+\mathrm{L})$

HRP-coupled AffiniPure

1:10,000 Jackson Immunoresearch

$715-036-150$

$F(a b ') 2$ fragment, anti rabbit

$\lg \mathrm{G}(\mathrm{H}+\mathrm{L})$

\section{III.10.4 Secondary Antibody for Fiber Assay}

\begin{tabular}{lllll} 
Antibody & Dilution & Producer & Catalogue nr. \\
\hline $\begin{array}{l}\text { Alexa-Fluor-488 goat anti } \\
\text { mouse }\end{array}$ & $1: 250$ & $\begin{array}{l}\text { Invitrogen, } \\
\text { Technologies }\end{array}$ & Life & A-11017 \\
$\begin{array}{l}\text { Alexa-Fluor-555 goat anti } \\
\text { mouse }\end{array}$ & $1: 250$ & $\begin{array}{l}\text { Invitrogen, } \\
\text { Technologies }\end{array}$ & Life & A-11003 \\
$\begin{array}{l}\text { Alexa-Fluor-647 goat anti } \\
\text { mouse }\end{array}$ & $1: 250$ & $\begin{array}{l}\text { Învitrogen, } \\
\text { Technologies }\end{array}$ & Life A-21236
\end{tabular}




\section{III.1 Cell culture}

\section{III.11.1 Cell lines}

Cell line

\begin{tabular}{|c|}
\hline U2OS \\
\hline HCT116 p53-/- \\
\hline H1299 \\
\hline p53 $3^{-/-}$MEF \\
\hline $\mathrm{p} 53^{-/-} \mathrm{Mdm}^{-/-} \mathrm{MEF}$ \\
\hline $\mathrm{p} 53^{-/-} \mathrm{Mdm} 2^{\mathrm{C} 462 \mathrm{~A} / \mathrm{C} 462 \mathrm{~A}} \mathrm{MEF}$ \\
\hline p53 ${ }^{-/-}$thymocytes \\
\hline $\mathrm{p} 53^{\operatorname{loxP}} \mathrm{MEF}$ \\
\hline
\end{tabular}

\section{Source}

human osteosarcoma cell line, p53-proficient, ATCC

human colon carcinoma cell line, p53-deficient (Bunz et al., 1998), ATCC

human non-small lung cell carcinoma cell line, homozygous deletion of p53 gene, ATCC

p53-deficient mouse embryonic fibroblasts with Mdm2 knock-out/ RING finger mutation knock-in; generated by Zhang lab, UNC Medial School, North Carolina, USA (Clegg et al., 2012)

isolation from animals at 3-4 weeks of age (Klusmann et al., 2016)

isolation from animals at E13.5 (Klusmann et al. 2016)

\section{III.11.2 Media and reagents for cell culture}

Reagent

Ciprofloxacin

DMEM GlutaMAX

DMEM powder

FCS

L-glutamine

PBS (tablets)

Penicillin/Streptomycin

Puromycin

RPMI 1640 w Hepes w/o Glut

Tetracyclin

Trypsin/EDTA

\section{Producer}

Bayer

Gibco, Life Technologies

Gibco, Life Technologies

Gibco, Life Technologies

Gibco, Life Technologies

Gibco, Life Technologies

Gibco, Life Technologies

Gibco, Life Technologies

Gibco, Life Technologies

Gibco, Life Technologies

Gibco, Life Technologies 
Dulbecco's Modified Eagle's Medium (DMEM)

\begin{tabular}{ll}
\hline DMEM powder & $10 \mathrm{~g} / \mathrm{l}$ \\
$\mathrm{NaHCO}_{3}$ & $3.7 \mathrm{~g} / \mathrm{l}$ \\
$\mathrm{HEPES}$ & $5.96 \mathrm{~g} / \mathrm{l}$
\end{tabular}

Dulbecco's Modified Eagle's Medium (DMEM) with supplements

\begin{tabular}{ll}
\hline DMEM & \\
FCS & $10 \%$ \\
Penicillin/Streptomycin (Pen/Strep) & $50 \mathrm{U} / \mathrm{ml}$ \\
L-glutamine & $200 \mu \mathrm{M}$ \\
Ciprofloxacin & $10 \mu \mathrm{g} / \mathrm{ml}$ \\
in ddH ${ }_{2} \mathrm{O}$ & \\
& \\
RPMI with supplements & \\
\hline RPMI & \\
FCS & $10 \%$ \\
Penicillin/Streptomycin (Pen/Strep) & $50 \mathrm{U} / \mathrm{ml}$ \\
L-glutamine & $200 \mu \mathrm{M}$ \\
in ddH & \\
\end{tabular}




\section{III.12 Bacteria}

III.12.1 Bacterial strains used for plasmid amplification

Bacteria strain

Source

$\mathrm{DH} 10 \mathrm{~B}^{\mathrm{TM}}$ chemically competent cells

Thermo Scientific

III.12.2 Bacteria growth medium

2YT medium

\begin{tabular}{ll}
\hline Tryptone & $1.6 \%$ \\
yeast extract & $1 \%$ \\
$\mathrm{NaCl}$ & $0.5 \%$ \\
& \\
2YT agar & \\
\hline YT agar & $15 \%$ \\
2YT medium & $100 \%$
\end{tabular}

III.13 Software and Databases

Name Producer

Adobe Illustrator, InDesign, Photoshop CC Adobe

AttoVision image acquisition software Becton Dickinson

Axio Vision

Zeiss

ChemoStar Imager

Intas

Excel

Microsoft

GraphPad Prism

GraphPad Software

Image Lab 5.2.1

Biorad

ImageJ

General Public License

NanoDrop Software

Peqlab 


\section{Methods}

Parts of methods in this chapter have previously been described in my Master's thesis (2015) as well as in the full version of the publication in chapter V.I published in Cell Reports (Klusmann et al., 2016).

\section{IV.1 Cell Biology}

\section{IV.1.1 Cell culture}

Cells were cultivated at $37^{\circ} \mathrm{C}, 5 \% \mathrm{CO}_{2}$, and humidified conditions in culture media listed in Table IV.1. For sub-cultivation, cells were washed with pre-warmed PBS and detached from the culture dish with $0.1 \%$ trypsin/EDTA and incubation at $37^{\circ} \mathrm{C}$ for 5 minutes. Once detached, the reaction was stopped by the addition of FCS-containing culture medium. Cells were re-seeded at dilutions between 1:2 to 1:10 three times per week. For experiments, cells were seeded into culture dishes at the appropriate density. All cell culture work was carried out under sterile conditions.

Table IV.1 Cell culture media

\section{Cell line}

U2OS

HCT116 p53-/-

$\mathrm{H} 1299$

p53 ${ }^{-/-}$MEF

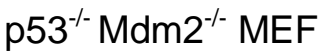

p55 ${ }^{-/-}$Mdm2 $2^{\mathrm{C} 462 \mathrm{~A} / \mathrm{C} 462 \mathrm{~A}} \mathrm{MEF}$

p53 $3^{-/-}$thymocytes

p53 ${ }^{\text {loxP }}$ MEF

\section{Culture medium}

DMEM with supplements

RPMI with supplements

DMEM with supplements

DMEM with Pen/Strep, L-glutamine, and sodium pyruvate

RPMI with supplements

DMEM Glutamax with supplements 


\section{IV.1.1.1 Isolation and sources of mouse embryonic fibroblasts}

Mouse embryonic fibroblasts (MEFs) were isolated from $\mathrm{p} 53^{\text {loxP }}$ mice (B6.129P2Trp53tm1Brn/J, Jackson Laboratories) at E13.5 (Jonkers et al., 2001). Littermates were used to prepare MEFs from a C57BI6N/129SV background (50\%-50\%, N2 Backcross). For this, abdominal parts of murine embryos at E13.5 were dissected and incubated in pre-warmed trypsin/EDTA for $30 \mathrm{~min}$ at $37^{\circ} \mathrm{C}$ and $5 \% \mathrm{CO}_{2}$. Subsequently, cells were isolated further by pipetting and transferred to $10 \mathrm{~cm}$ cell culture dishes with pre-warmed DMEM medium with supplements but without antibiotics for 6 hours. Medium is changed once more and cells kept at $37^{\circ} \mathrm{C}, 5 \% \mathrm{CO}_{2}$ overnight. Cells were split once before experiments and freezing for storage in liquid nitrogen.

MEFs from p53-/- , p53-/- Mdm2-/-, and p53-/- Mdm2-C462A/C462A mice with a C57BL/6 background were obtained from Y. Zhang, University of North Carolina (Itahana et al., 2007; Wienken et al., 2016). MEF cultures were maintained in DMEM supplemented with 10\% FCS, L-glutamine, sodium pyruvate, and antibiotics.

\section{IV.1.1.2 Isolation of murine Thymocytes}

Thymocytes were isolated from three to four weeks old Trp53tm1Tyj mice (Jackson Laboratories), carrying a deletion of the p53-encoding gene (Jacks et al., 1994). The thymus was isolated and strained through a $40 \mu \mathrm{M}$ mesh. Isolated cells were washed with PBS and transferred into pre-warmed RPMI 1640 (Invitrogen/Gibco, Life Technologies) supplemented with $10 \%$ FCS. Cells were used for experiments immediately after isolation.

All experiments were carried out in full agreement with the Göttingen University Animal Care Committee and the Institutional Guidelines for Humane Use of Animals in Research.

\section{IV.1.2 Transient transfections}

For the transient knockdown of gene expression in human cells, a reverse transfection protocol was followed using the cationic liposome formulation Lipofectamine 2000 (in chapter V.I) and Lipofectamine3000 (in chapter V.II). For this, a transfection mix with a final siRNA concentration of $10 \mathrm{nM}$ was prepared by separately incubating siRNA (Solution A.1) and Lipofectamine (Solution B; Table IV.1.2) in DMEM without supplements, before combining the two solutions and an additional 15 minute incubation. Cells were seeded with the transfection mix into medium containing supplements. Culture medium was exchanged after 24 hours and experiments were carried out 48 hours post siRNA transfection. 
For the ectopic expression of plasmid DNA in chapter V.II, a forward transfection protocol was used according to manufacturer's instructions. For this, a transfection mix was prepared from $2 \mu \mathrm{g}$ of plasmid DNA and the P3000 reagent (solution A.2), and a second mix containing Lipofectamine3000 (solution B) in DMEM without supplements. They were combined (solutions A.2 \& B) and incubated for 15 minutes. The transfection mix was added drop-wise onto adherent cells and experiments carried out 30 hours post transfection.

Table IV.2 Transfection mix per reaction

\begin{tabular}{|c|c|c|}
\hline Solution & Components & Protocol \\
\hline A.1 (siRNA) & $\begin{array}{l}0.5 \mu \mathrm{l} 50 \mu \mathrm{M} \text { siRNA } \\
250 \mu \mathrm{l} \text { DMEM }\end{array}$ & \\
\hline & $\begin{array}{l}2 \mu \mathrm{g} \text { plasmid DNA } \\
5 \mu \mathrm{l} 3000\end{array}$ & \\
\hline A.2 (DNA) & 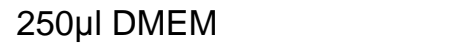 & \\
\hline B & 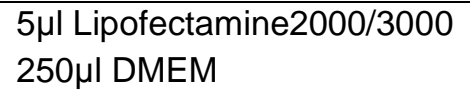 & vortex \\
\hline Transfection mix & Solutions A and B & vortex, incubate $10^{\prime} \mathrm{RT}$ \\
\hline
\end{tabular}

\section{IV.1.3 Chemical Treatments}

Pharmacological inhibitors used for cell treatments were dissolved in $\mathrm{H}_{2} \mathrm{O}$ or DMSO and aliquots prepared according to manufacturer's instructions. Treatments were prepared by adding the drug to pre-warmed medium as indicated in Table IV.1.3. For control samples, the respective solvent was added instead of the drug

Table IV.3 Drug concentrations and solvents

\begin{tabular}{lll} 
Name & Stock concentration & Solvent \\
\hline Gemcitabine & $64 \mathrm{mM}, 126.8 \mu \mathrm{M}$ & $\mathrm{H}_{2} \mathrm{O}$ \\
Nutlin-3a & $20 \mathrm{mM}$ & $\mathrm{DMSO}$ \\
Hydroxyurea & $1 \mathrm{M}$ & $\mathrm{H}_{2} \mathrm{O}$ \\
PHA-766491 & $10 \mathrm{mM}$ & $\mathrm{DMSO}$ \\
EPZ6438 & $10 \mathrm{mM}$ & $\mathrm{DMSO}$ \\
DZNep & $10 \mathrm{mM}$ & DMSO \\
DRB (5,6-Dichlorobenzimidazole 1- $\beta$-D-ribofuranoside) & $50 \mathrm{mM}$ & DMSO \\
LDC067 & $20 \mathrm{mM}$ & DMSO \\
PTC-209 & $20 \mathrm{mM}$ & DMSO \\
\hline
\end{tabular}




\section{IV.1.4 Cell Lysates for SDS-PAGE Analysis}

For protein analysis via immunoblot, crude cell lysates were prepared from cells grown in 6 -well dishes. All steps of harvesting were performed on ice and with pre-cooled solutions to minimise protein degradation. Cells were washed twice with ice-cold PBS and detached from the culture dish by using a $16 \mathrm{~cm}$ cell scraper. Detached cells were resuspended in 75-100 $\mu$ l cell lysis buffer (Table III.4) and sonicated for 10 minutes at high power and 30 second intervals. Samples were subsequently stored at $-80^{\circ} \mathrm{C}$.

\section{IV.1.5 Cell synchronisation}

For synchronization of cells at the G1/S transition in chapter V.I, U2OS cells were incubated with $2 \mathrm{mM}$ thymidine in DMEM with supplements for $16 \mathrm{~h}$, then washed and incubated in DMEM+ for 8h, and then again incubated with $2 \mathrm{mM}$ thymidine for $16 \mathrm{~h}$ (double-thymidine block). For release, thymidine was removed by washing and incubation with DMEM+.

\section{IV.1.6 Flow cytometry}

For cell cycle analysis, cells were fixed with ethanol over night. Subsequently, samples were centrifuged and the pellet rehydrated with PBS for 10 minutes. After centrifugation, the cell pellet was resuspended in PBS with RNase A $(200 \mu \mathrm{g} / \mathrm{ml})$ and incubated at $37^{\circ} \mathrm{C}$ for 30 minutes. Propidium iodide staining was performed with a $30 \mu \mathrm{g} / \mathrm{ml}$ solution for $5-15$ minutes at room temperature in the dark. Flow cytometry was performed using the Guava PCA 96 System (Millipore). The percentage of cells in each phase of the cell cycle was determined using the Guava Express Pro software.

For detection of mitochondrial reactive oxygen species, live cells were incubated with $5 \mu \mathrm{M}$ MitoSOX ${ }^{\mathrm{TM}}$ Red Mitochondrial Superoxide Indicator in FACS binding buffer (Table III.4) at $37^{\circ} \mathrm{C}$ and $5 \% \mathrm{CO}_{2}$ for 40 minutes. After washing with PBS $\left(37^{\circ} \mathrm{C}\right)$ twice, the cells were detached with $0.5 \%$ trysin/EDTA, followed by flow cytometry as described above.

\section{IV.1.7 High-Content Immunofluorescence Microscopy}

Cells were grown in 96-well imaging plates (Becton Dickinson) for $24 \mathrm{~h}$ and treated with $10 \mu \mathrm{M}$ Nutlin-3a for 6,12 , and 24 hours. For the last 2 hours, $5 \mu \mathrm{M} 5$-ethynyl-2'deoxyuridine (EdU) was added to the media. After fixation in $4 \%$ paraformaldehyde/PBS, cells were permeabilized with $0.5 \%$ Triton-X100/PBS. Actively replicating cells were detected by using the Click-iT EdU Alexa488 HCS assay kit (Life Technologies, C10351). 
Hoechst staining was performed to detect cell nuclei. Automated microscopy was performed by using a Pathway HT Cell Imaging System in conjunction with the AttoVision image acquisition software (Becton Dickinson) and a single cell based image analysis. Quantification of DNA replication was determined in quadruplicate by backgroundcorrected EdU signal intensity.

\section{IV.1.8 Quantitative image-based cytometry (QIBC)}

Quantitative image-based cytometry (chapter V.II) was carried out and described by Federico Teloni and Matthias Altmeyer (University of Zurich) with reagents indicated.

\section{IV.1.8.1 EdU incorporation for QIBC}

For pulsed EdU (5-ethynyl-2'-desoxyuridine) (Thermo Fisher Scientific) incorporation, cells were incubated for 20 minutes in medium containing $10 \mu \mathrm{M}$ EdU. The Click-iT EdU Alexa Fluor Imaging Kit (Thermo Fisher Scientific) was used for EdU detection. EdU-positive cells as detected by QIBC were considered the S-phase population.

\section{IV.1.8.2 Quantitative image-based cytometry (QIBC) analysis}

GFP-RNAseH1 D210N cells were grown on sterile $12 \mathrm{~mm}$ glass coverslips, pre-extracted in ice-cold $0.2 \%$ Triton X-100 (Sigma-Aldrich) in PBS for 2 minutes on ice to wash out detergent-sensitive, non-chromatin-bound proteins, washed twice in PBS, and fixed in $3 \%$ formaldehyde in PBS for 15 minutes at room temperature. Primary and secondary antibodies (Alexa fluorophores, Life Technologies) were diluted in filtered DMEM containing 10\% FBS and $0.02 \%$ Sodium Azide. Antibody incubations were performed for 2 hours (primary antibodies) or 1 hour (secondary antibodies) at room temperature. After antibody incubations, coverslips were washed once with PBS and incubated for 10 minutes with PBS containing 4',6-Diamidino-2-Phenylindole Dihydrochloride (DAPI, 0.5 $\mu \mathrm{g} / \mathrm{ml}$ ) at room temperature to stain DNA. Coverslips were mounted on $5 \mu$ l Mowiol-based mounting media (Mowiol 4.88 (Calbiochem) in Glycerol/TRIS). H2AX Phospho S139 antibody (mouse, Biolegend 613401, 1:1000) was used to detect DNA damage signaling. Automated multichannel wide-field microscopy for QIBC was performed on an Olympus ScanR Screening System equipped with an inverted motorized Olympus IX83 microscope, a motorized stage, IR-laser hardware autofocus, a fast emission filter wheel with single band emission filters, and a digital monochrome Hamamatsu ORCA-FLASH 4.0 V2 sCMOS camera (2048 x 2048 pixel, 12 bit dynamics) as described previously (Pellegrino et al., 2017). For each condition, image information of large cohorts of cells was acquired under non-saturating conditions with a UPLSAPO 10x (NA 0.4) objective. Identical settings were applied to all samples within one experiment. Images were 
analyzed with the Olympus ScanR Image Analysis Software Version 2.5.1, a dynamic background correction was applied and nuclei segmentation was performed using an integrated intensity-based object detection module using the DAPI signal. All downstream analyses were focused on properly detected interphase nuclei or mitotic chromosomes containing a 2C-4C DNA content as measured by total and mean DAPI intensities. Fluorescence intensities were quantified and are depicted as arbitrary units. Color-coded scatter plots of asynchronous cell populations were generated with Spotfire data visualization software (TIBCO). Within one experiment, similar cell numbers were compared for the different conditions. Representative scatter plots and quantifications of independent experiments, typically containing several thousand cells, are shown. 


\section{IV.2 Biochemistry}

\section{IV.2.1 Bicinchoninic acid assay}

Relative protein concentrations were determined colorimetrically using a bicinchoninic acid assay. In this method, the reduction of $\mathrm{Cu}^{2+}$ to $\mathrm{Cu}^{+}$by protein in alkaline medium is made visible through the addition of bicinchoninic acid which chelates one cuprous ion $\left(\mathrm{Cu}^{+}\right)$and forms a purple-coloured reaction product (Smith et al., 1985). Protein samples were adjusted to equal protein concentrations with RIPA buffer and 6x Laemmli buffer was added. Samples were subsequently used for SDS-PAGE or stored at $-80^{\circ} \mathrm{C}$.

\section{IV.2.2 Separation of proteins by SDS-PAGE}

SDS-PAGE (Sodium dodecyl sulfate polyacrylamide gel electrophoresis), developed by Shapiro et al. in 1967, is a method for separating proteins according to their electrophoretic mobility (Shapiro et al., 1967). For this, proteins are denatured by incubation at $95^{\circ} \mathrm{C}$ for 5 minutes in the presence of SDS.

The anionic detergent SDS binds to all proteins and confers a negative charge to them so that all proteins migrate towards the anode, when an electric field is applied. As a result, proteins migrate relative to their molecular weight with little influence of internal charges. The gel used for electrophoresis, is divided into a stacking gel with $5 \%$ acrylamide and $\mathrm{pH}$ 6.8 and the resolving gel with $12 \%$ acrylamide and $\mathrm{pH}$ 8.8. Larger pores in the stacking gel allow the proteins to be focused between the leading chloride and trailing glycine ions that "stack" the proteins at the edge of the resolving gel. The smaller pore size of the resolving gel allows the actual size-dependent separation of the proteins.

SDS-PAGE gels were casted between two clean glass plates. For this, the resolving gel was cast first and overlaid with 2-propanol for an even surface. Once polymerised, the propanol was discarded and the stacking gel cast on top. A 10 - or 15 -well comb was inserted and left to polymerise.

Adjusted protein samples $(20-40 \mu l)$ were loaded into wells of the stacking gel together with a pre-stained protein ladder Electrophoresis was carried out with a constant voltage of $80 \mathrm{~V}$ for stacking and $120 \mathrm{~V}$ for separation. 
Table IV.4 Composition of gels for SDS-PAGE

\begin{tabular}{lll} 
Component & stacking gel & resolving gel \\
\hline acrylamide-bisacrylamide & $5 \%$ & $12 \%$ \\
$1 \mathrm{M}$ Tris- $\mathrm{HCl}, \mathrm{pH} 6.8$ & $126 \mathrm{mM}$ & \\
$1.5 \mathrm{M}$ Tris- $\mathrm{HCl}, \mathrm{pH} 8.8$ & & $375 \mathrm{mM}$ \\
$10 \%$ SDS & $0.1 \%$ & $0.1 \%$ \\
$10 \%$ APS & $0.1 \%$ & $0.1 \%$ \\
TEMED & $0.3 \%$ & $0.4 \%$ \\
\hline
\end{tabular}

\section{IV.2.3 Immunoblotting}

Separated proteins in the SDS-PAGE gel were further analysed by immunoblotting, a technique first used by Renart et al. in 1979 and further developed by Towbin et al. in the same year (Renart et al., 1979; Towbin et al., 1979).

With this method, proteins are transferred from their position on the gel onto a nitrocellulose membrane (Bittner et al., 1980). For the transfer the membrane was laid on top of the gel and covered by Whatman paper and sponges on both sides. The stack was placed into a tank blot chamber filled with Western Blot Transfer Buffer (Table III.4) and an electric field with a constant voltage of $90-100 \mathrm{~V}$ was applied for $90-180$ minutes at $4^{\circ} \mathrm{C}$. The membrane was subsequently stained with Ponceau S solution (Table III.4) to check for protein transfer efficiency.

\section{IV.2.4 Immunostaining}

In order to detect specific proteins on the membrane, a two-component antibody system was used. In this, the primary antibody is specific for the protein or post-translational modification of interest, whereas the secondary antibody recognises the constant region of the primary antibody which is specific for the animal host in which the antibody was raised. The conjugation of the secondary antibody to horseradish peroxidase (HRP) allows the detection of antibody binding by chemiluminescence. Upon the addition of a substrate solution, HRP catalyses the oxidation of luminol by peroxide. The light emitted during this reaction can be captured by a camera and is visualised as bands with varying intensity according to protein levels with the Intas ChemoStar Imager Software (chapter V.I) and the Image Lab 5.2.1 Software by Biorad (chapter V.II).

To avoid unspecific binding of the antibody to the protein membrane, the membrane was first incubated in 5\% milk in TBS-T (Table III.4) for one hour at room temperature. 
Subsequently, the primary antibody incubation was carried out at $4^{\circ} \mathrm{C}$ overnight using concentrations stated in Table III. 10.1. Afterwards, the membrane was washed three times in TBS-T for 10 minutes before incubation with the corresponding secondary antibody for one hour at room temperature (Table III. 10.3). Before visualisation, the membrane was again washed three times in TBS-T for 10 minutes.

Two substrate solutions were used depending on signal intensity - Immobilon Western HRP Substrate Peroxide Solution for intense signals and SuperSignal West Femto Maximum Sensitivity Substrate for weaker signals. 


\section{IV.3. Molecular Biology}

\section{IV.3.1 RNA extraction, reverse transcription, and real time quantitative PCR}

Total RNA was extracted from cells using TRIzol®. mRNA was reverse-transcribed using oligo-dT and random hexameric primers, followed by qRT-PCR analysis using SYBR Green (Invitrogen). Gene expression levels were normalized to the mRNA encoding 36B4, and the analysis was conducted using the $\triangle \triangle \mathrm{Ct}$ method. qRT-PCR primer sets were chosen as follows:

Table IV.5 Primer sequences for gene expression studies in human cells

\begin{tabular}{|l|l|}
\hline Gene name & Primer sequence \\
\hline 36B4 & $\begin{array}{l}\text { For- GAT TGG CTA CCC AAC TGT TG } \\
\text { Rev - CAG GGG CAG CAG CCA CAA A }\end{array}$ \\
\hline CDKN1A/p21 & $\begin{array}{l}\text { For- CCT GGC ACC TCA CCT GCT CTG CTG } \\
\text { Rev- GCA GAA GAT GTA GAG CGG }\end{array}$ \\
\hline
\end{tabular}

\section{IV.3.2 RNA sequencing}

For RNA-sequencing, the quality of total RNA was determined using the Bioanalyzer 2100 from Agilent. All samples analyzed exhibited a RNA Integrity Number > 8. Library preparation was conducted using the TruSeq RNA LT SamplePrep Kit, starting from 1000 ng of total RNA. Barcodes for sample preparation were used according to the indications given by the protocol. Accurate quantitation of cDNA libraries was performed with the QuantiFluor ${ }^{\mathrm{TM}} \mathrm{dsDNA}$ System (Promega). The size range of final cDNA libraries was determined applying the DNA 1000 chip on the Bioanalyzer 2100 (Agilent; 290-310 bp). cDNA libraries were amplified and sequenced via cBot and HiSeq 2000 (Illumina; SR, $1 \times 50 \mathrm{bp}, 6 \mathrm{~Gb} / \mathrm{sample} \mathrm{ca}$. 30 million reads per sample). Sequence images were transformed with Illumina software BaseCaller to bcl files, which were demultiplexed to fastq files with CASAVA (version 1.8.2). Quality check was performed via FastQC (version 0.10.1, Babraham Bioinformatics). Fastq files were mapped to the human reference transcriptome (UCSC hg19) using Tophat (Galaxy Version 0.9; Kim et al., 2013). Read counts for each sample and each gene were aggregated using a htseq-count (Anders et al., 2014). DESeq2 (version 1.10.1) was used for measuring differential expression (Love et al., 2014). RNA library preparation and sequencing was done by the Transcriptome Analysis Laboratory (TAL, Göttingen). 


\section{IV.3.2 DNA Fiber Assay}

DNA fiber assays were used to study replication speed and progression in cells subjected to different treatments. This method, first published by Jackson et al. in 1998, involves two labelling pulses with the nucleoside analogues 5-Chloro-2'-deoxyuridine (CldU) and 5lodo-2'-deoxyuridine (IdU; Jackson \& Pombo, 1998). Their incorporation into newly replicated DNA provides a mark that can be used for immunostaining at a later stage.

After treatment and labelling (cf. IV.3.5.1) of cells in culture and subsequent harvesting, cells were lysed and their DNA spread out on glass plates. Fixed slides can subsequently be used for immunostaining by denaturation, blocking, and antibody incubation steps. Both, CldU and IdU are specifically recognised by anti-BrdU antibodies (CldU by rat-aBrdU and IdU by mouse-a-BrdU). Fluorescent secondary antibodies allow visualisation of incorporated CldU and IdU with a fluorescent microscope. Structures observed under the microscope and their identity, are indicated in Fig. VI.3.1.

Pre-treatment of the cells before labelling will affect both CldU and IdU label length and the effect of the treatment given can be analysed by comparing the average replication fork progression of treated and control samples.

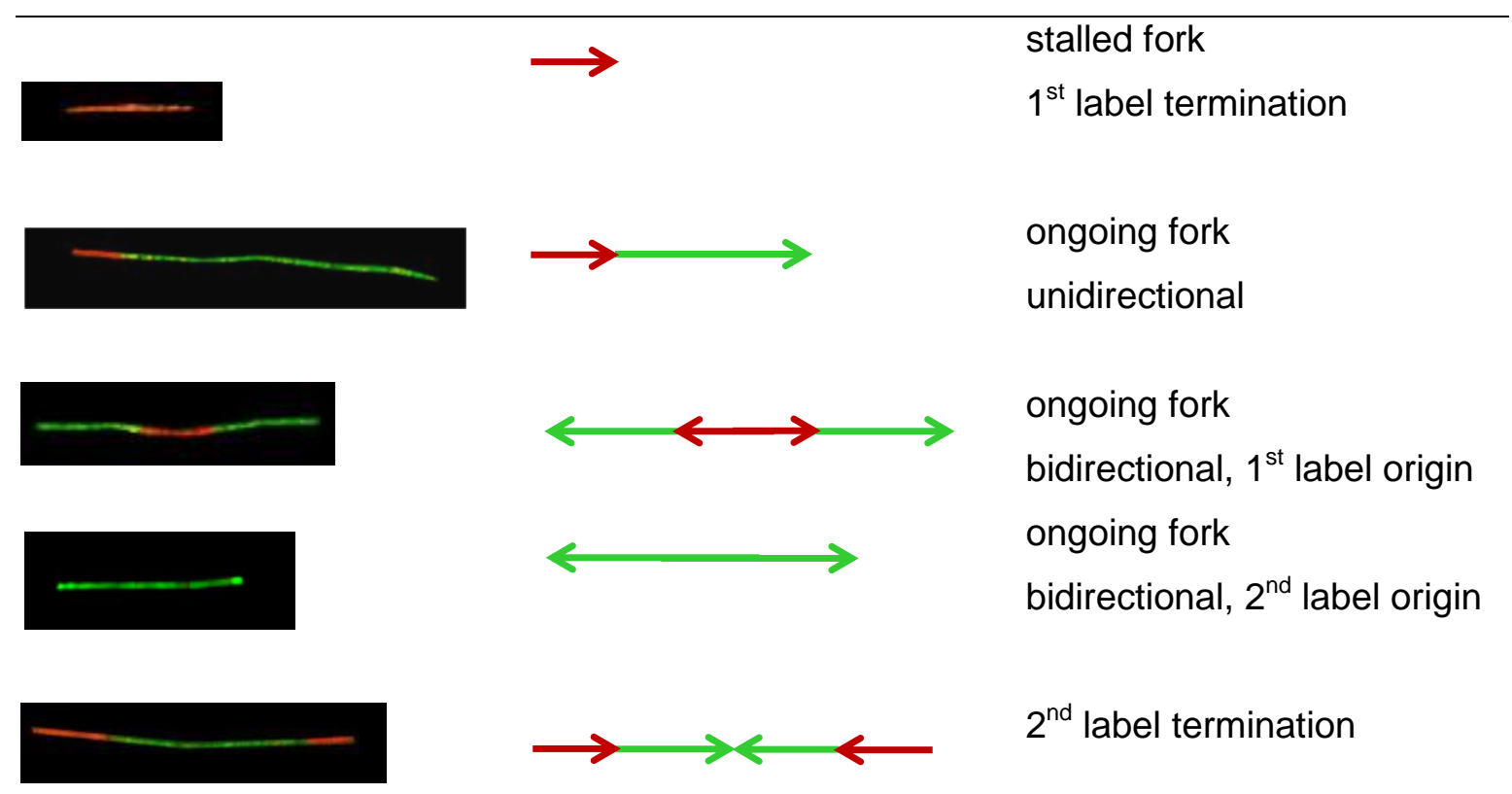

Fig. IV.3.1 Representative images of DNA fibers and a schematic diagram of structures observed in fiber assays after a two-label protocol

CldU labelled DNA was detected with a red (Alexa555) and IdU with a green (Alexa488) fluorescent antibody. Only unidirectional ongoing forks were used for replication speed analysis. 


\section{IV.3.2.1 Pulse Labelling}

For DNA fibre assays, cells were seeded into $25 \mathrm{~cm}^{2}$ cell culture flasks at a density of 500,000 cells per flask for when labelling was performed the next day. For siRNA experiments, 400,000 cells per flask were seeded 48 hours prior to fiber assay experiments. Media containing CldU $(25 \mu \mathrm{M})$ and IdU $(25-250 \mu \mathrm{M})$ were incubated at $37^{\circ} \mathrm{C}$, $5 \% \mathrm{CO}_{2}$ and humidity for at least 12 hours prior to use. Drugs and inhibitors were added to both media in a 6-well plate and mixed well just before the labelling procedure and added at different points during the experiment depending on the experiment.

\section{IV.3.2.1.1 Labelling for fork progression analysis}

For pulse labelling, CldU containing medium was applied to the cells for 20 minutes, aspirated, and IdU medium applied for 1-2 hours as indicated in the schematic labelling diagram in the figures. IdU was used at a tenfold concentration compared to CldU in chapter V.I but at equal concentrations in chapter V.II as we identified that it is not necessary to outcompete the first label with a higher concentration of the second one.

\section{IV.3.2.1.2 Labelling for fork processivity analysis}

In order to assess fork stalling, alternating labels of CldU and IdU were incorporated to obtain a striped pattern. For this, both CldU and IdU containing media were prepared at the same concentration of $25 \mu \mathrm{M}$. A first long label was incorporated by incubation with CldU-containing medium for one hour. Subsequently, IdU and CldU media were changed six more times after an incubation time of 10-15 minutes each, to result in an overall labelling time of 2-2.5 hours and seven incorporated labels (Fig. IV.3.2)
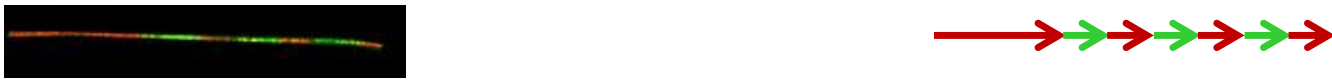

Fig. IV.3.2 Representative image of a fiber observed in the fork stalling assay with seven alternating labels of CIdU and IdU

CldU labelled DNA was detected with a red (Alexa555) and IdU with a green (Alexa488) fluorescent antibody. The number of labels was counted and used for the analysis of fork processivity. 


\section{IV.3.2.2 Harvest of labelled cells}

After the labelling procedure, cells were harvested by washing twice with cold PBS and scraping in $2 \mathrm{ml}$ cold PBS. A cell pellet obtained by centrifugation at $4^{\circ} \mathrm{C}$ and $4,400 \mathrm{rpm}$ for 5 minutes was resuspended to a final concentration of 500,000 cells per $\mathrm{ml}$ in PBS.

\section{IV.3.2.3 Spreading}

Cells were spread onto a Superfrost glass slide by applying a $2 \mu \mathrm{l}$ drop of cell suspension on top of the slide and airdrying for about 3 minutes. Cells were lysed by applying DNA Fiber spreading buffer (Table III.4) and incubation for 2 minutes at room temperature.

Cells in lysis buffer were spread over the slide by tilting it slightly for the drop to run down slowly (about $3 \mathrm{~cm} / \mathrm{min}$ ). Upon drying under a fume hood cells burst and release DNA at their relative position. Finally, the slides were fixed in a Fiber assay fixative solution (Table III.4) for 10 minutes at room temperature before storage at $4^{\circ} \mathrm{C}$ for up to one month.

\section{IV.3.2.4 Immunostaining}

Fixed slides were rehydrated by incubation with $\mathrm{ddH}_{2} \mathrm{O}$ twice for 5 minutes. Next, the samples were denatured to obtain single stranded DNA by equilibrating the slides with $2.5 \mathrm{M} \mathrm{HCl}$ for 5 minutes and incubation at room temperature for 80 minutes. To remove all acid, the samples were washed twice with PBS and subsequently incubated with Blocking solution (Table III.4) twice for 5 minutes.

Prior to immunostaining, the slides were blocked for one hour to avoid unspecific antibody binding. Primary antibodies recognising epitopes on CldU and IdU specifically, were applied to slides in 150 $\mu$ l blocking solution with a 1:200-1000 dilution (Table III.10.2) and incubated at $37^{\circ} \mathrm{C}$ for one hour. Samples were rinsed with PBS and fixed in a $4 \%$ paraformaldehyde solution for 10 minutes. The slides were again rinsed with PBS and incubated with blocking solution three times for 5 minutes. Fluorescently labelled secondary antibodies AlexaFluor488 goat-anti-mouse and AlexaFluor555 goat-anti-rat (Table III. 10.4) recognise primary antibodies from rat origin and were applied to slides in $150 \mu \mathrm{l}$ with a dilution of 1:250 and incubated for 2 hours at room temperature.

Final washing steps of rinsing once with PBS, washing twice with blocking solution for 5 minutes, rinsing with PBS and water were conducted prior to mounting with Vectashield mounting medium. 


\section{IV.3.2.5 Microscope analysis}

Each slide was analysed with an Axio Scope A1 microscope (Zeiss) with filters for 488 and 555nm, a EC Plan-Neofluar 40x objective (Zeiss) and an Axio Cam MRc/503 camera (Zeiss). Eight to twenty images were taken from all parts of the slide and from at least two slides per sample. Measurements of fiber and label length as well as their occurrence were carried out using Image $\mathrm{J}$ and its cell counter plugin (Kurt de Vos, University of Sheffield, UK). All data was further processed using Microsoft Excel and GraphPad Prism.

\section{IV.4 Statistical Analysis}

Statistical analysis was carried out using the GraphPad Prism Software (Versions 6 and 7). Statistical significance was assessed by applying the unpaired 2-sided Student's t-test and a cutoff value of $p=0.05$, under which differences are counted as significant. $P$-values calculated for each experiment are indicated in result figures. 


\section{V.I Publication}

\section{p53 activity results in DNA replication fork processivity}

Ina Klusmann ${ }^{1}$, Sabrina Rodewald ${ }^{1}$, Leonie Müller ${ }^{1}$, Mascha Friedrich ${ }^{1}$, Magdalena

Wienken ${ }^{1}$, Yizhu Li ${ }^{1}$, Ramona Schulz-Heddergott ${ }^{1}$, and Matthias Dobbelstein ${ }^{1,2}$

1) Institute of Molecular Oncology, Göttingen Center of Molecular Biosciences (GZMB), University Medical Center Göttingen, D-37077 Göttingen, Germany

2) Lead Contact. Correspondence and requests for materials should be addressed to M. D. (e-mail:mdobbel@uni-goettingen.de)

Keywords: p53, DNA replication, gemcitabine, Mdm2, Nutlin-3a, DNA fiber assays, DNA damage response, murine embryonic fibroblasts, thymocytes

Cell Reports Volume 17, Issue 7, p1845-1857, 8 November 2016

https://doi.org/10.1016/j.celrep.2016.10.036

Supplemental Tables can be found in the online version of this publication.

\section{Contribution to publication}

Data analysis for RNA-Seq in Fig. V.I.1 and Suppl. Fig. V.I.7.1 as well as experiments and data analysis for Fig. V.I.2, Fig. V.I.3, Fig. V.I.4, Fig. V.I.5A-D, Supp. Fig V.I.7.1B, Supp. Fig. V.I.7.2A, Supp. Fig. V.I.7.2C-I, Supp. Fig. V.I.7.3, Supp. Fig. V.I.7.4A-F, Supp. Fig. V.I.7.4I, Suppl. Fig. V.I.7.5, Suppl. Fig. V.I.7.6F, Suppl. Fig. V.I.7.7A, all three Supplemental Tables as well as Figure layout and contributions to the manuscript by IK. 


\section{V.I.1 Abstract}

p53 induces cell death upon DNA damage, but this may not confer all of its tumor suppressor activity. We report that p53 activation enhances the processivity of DNA replication, as monitored by multi-label fiber assays, whereas removal of p53 reduces fork progression. This was observed in tumor-derived U2OS cells, but also in murine embryonic fibroblasts with heterozygous or homozygous p53 deletion, and in freshly isolated thymocytes from mice with differential p53 status. Mdm2, a p53-inducible gene product, similarly supported DNA replication even in p53-deficient cells, suggesting that sustained Mdm2-expression is at least one of the mechanisms allowing p53 to prevent replicative stress. Thus, p53 helps to protect the genome during S phase, by preventing the occurrence of stalled or collapsed replication forks. These results expand p53's tumorsuppressive functions, adding to the ex-post model (elimination of damaged cells) an exante activity, i.e. the prevention of DNA damage during replication. 


\section{V.I.2 Introduction}

No other gene is as frequently mutated across most tumor species as TP53. Thus, p53 must prevent tumor initiation and/or progression. Current concepts summarize the function of p53 largely as a mediator of cell death or permanent cell cycle arrest whenever cells suffer intolerable stresses, most notably when DNA damage occurs. DNA damage induces the activation of p53 as a transcription factor. Many of the p53-inducible genes mediate apoptosis. This ensures the elimination of cells that had suffered extensive DNA damage, conceivably avoiding the accumulation of cells with heavily mutated DNA that might otherwise give rise to malignant growth. Thus, the initial designation of p53 as a "guardian of the genome" (Lane, 1992) only applies to a whole organism, when damaged cells are eliminated to avoid danger to the whole body. From the perspective of a single cell, the "guardian" has a destructive role. According to currently accepted models, p53 is acting largely "ex post" by destroying damaged cells, but not "ex ante" to avoid DNA damage in the first place. Although DNA repair has now become another well acknowledged activity promoted by p53 (Bieging et al., 2014), this does still not imply that p53 actually prevents DNA damage, rather than merely reacting to it.

However, some observations at least argue that p53 not only eliminates cells with damaged DNA but exerts some of its tumor suppressive activity by precluding such damage. Mice with a constitutive deletion of p53 develop largely normally. At 4-6 months of age, however, tumors occur (Donehower et al., 1992). In mice with switchable p53 alleles, p53 function can be turned on and off at will, allowing the establishment of time windows during which p53 is essential or dispensable for tumor suppression (Martins et al., 2006). Using this system, established lung tumors were only temporarily halted by reintroducing wild type p53 (Junttila et al., 2010), arguing that p53 may have a more important role in preventing the formation of tumor cells rather than destroying them ex post. Investigating mouse strains with targeted deletions of key p53 target genes further challenged our current concept of p53-mediated tumor suppression. Even when the major mediator of cell cycle arrest, CDKN1A/p21, and the key proapoptotic gene product, BBC3/Puma, were both eliminated, p53 was still capable of suppressing T cell lymphomas that otherwise occur almost without exception when p53 itself is deleted (Valente et al., 2013). Similarly, an acetylation-deficient p53 mutant that is largely unable to induce cell cycle arrest or apoptosis can still suppress T cell lymphomas in mice (Li et al., 2012). Thus, neither the proapoptotic nor the cell cycle regulatory function of p53 may be key to its tumor suppressive activity. 
In further support of a protective function of p53 towards individual cells, the elimination of p53 does not always enhance cell survival. Rather, removing p53 in the colon cancerderived cell line HCT116 increases the sensitivity of cells towards certain chemotherapeutics, most notably doxorubicin and cisplatin (Bunz et al., 1999). The sensitivity of p53-deficient cells towards topoisomerase inhibitors was recently characterized in depth by a drug screen and mechanistic analysis (Yeo et al., 2016). siRNA screens revealed that the depletion of some gene products decreases the viability of p53-/- cells to a higher degree than their p53-proficient counterparts. These genes are involved in nucleotide synthesis, e.g. UMPS (Bartz et al., 2006), DNA replication, e.g. Geminin (Krastev et al., 2011), or DNA repair by homologous recombination, e.g. BRCA1 and RAD51 (Xie et al., 2012). Thus, p53 may support cell survival by preventing the accumulation of DNA damage during challenges to DNA replication.

Our previous results indicate that p53 can protect cells towards chemotherapeutics. These drugs still represent the mainstay of cancer treatment (Dobbelstein and Moll, 2014), and the induction of replicative stress is a key mechanism of many chemotherapies (Dobbelstein and Sørensen, 2015). When we activated p53 using the pharmacological Mdm2 inhibitor Nutlin-3a (Nutlin; Vassilev et al., 2004), we observed decreased cytotoxicity of gemcitabine (Kranz and Dobbelstein, 2006), UV-irradiation (Kranz et al., 2008), and Wee1 inhibitors ( $\mathrm{Li}$ et al., 2015). Initially, we ascribed this mostly to the cell cycle arrest function of $\mathrm{p} 53$, keeping the cells out of $S$ phase.

Here, we investigated whether p53 can influence the accumulation of DNA damage and replicative stress during $S$ phase. However, previous reports suggested that p53 activity might be attenuated during DNA replication. Most of these experiments used hydroxyurea, an inhibitor of ribonucleotide reductase, to arrest the cells in $S$ phase. Under such circumstances, the induction of CDKN1 A by p53 appeared reduced (Gottifredi et al., 2001; Mattia et al., 2007). However, this does not exclude an activity of p53 when cells proceed through $S$ phase rather than being arrested in it.

We show that p53 induces most of its target genes during $S$ phase and increases the processivity of DNA replication. The absence of p53 causes replicative stress. This was observed not only in tumor-derived cell lines, but in fibroblasts and thymocytes from mice, comparing p53-proficient and-deficient genotypes. Thus, p53 protects the genome by ensuring undisturbed progression of DNA replication forks. 


\section{V.I.3 Results}

\section{V.I.3.1 p53 is capable of inducing the majority of its target genes during S phase}

Previous reports have suggested that p53 activity might be attenuated while cells are replicating their DNA, but this was mostly studied in the context of exogenous replicative stress (Gottifredi et al., 2001; Mattia et al., 2007). To address this during ongoing, unperturbed S phase, we synchronized U2OS cells using a double thymidine block (Bootsma et al., 1964; Xeros, 1962). We then compared the expression of a bona fide p53 target gene, CDKN1A/p21, between asynchronously proliferating cells, cells that were arrested by a thymidine block, and cells that had been released from the block to enter $S$ phase. In each condition, the cells were treated with the Mdm2-inhibitor Nutlin-3a (Nutlin) to induce p53. Nutlin did not preclude the onset of $S$ phase upon release from the thymidine block (Fig. V.I.1A). We found that CDKN1A/p21 mRNA levels were enhanced by Nutlin under all three conditions but did not grossly differ between asynchronous, arrested and released cells (Fig.V.I.1B). When analyzing p21 protein levels by immunoblot, we observed the induction by Nutlin under all three conditions again, and p21 was even more abundant in the cells that were released to enter $S$ phase (Suppl. Fig.V.I.7.1A). This argues against the view that p53 activity might be impaired during DNA replication.

A

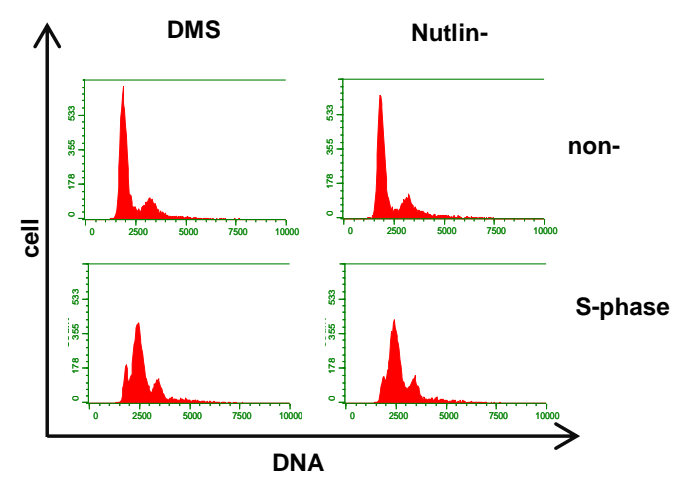

B

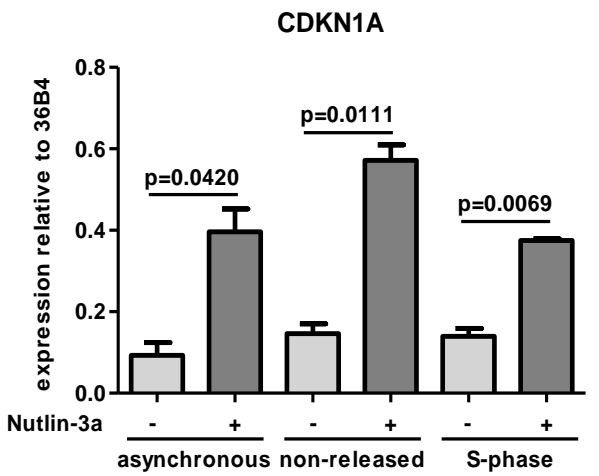

Fig. V.I.1 p53-mediated induction of genes during S-phase.

(A) Thymidine block and release result in comparable cell cycle distribution, independent of Nutlin pretreatment. U2OS cells were subjected to a double thymidine block. Four hours before release from the block (or maintaining the block), $10 \mu \mathrm{M}$ Nutlin (control: DMSO alone) was added. When the block was removed, the previous concentration of Nutlin was maintained, followed by further incubation for $4 \mathrm{~h}$. The cellular DNA content was determined by propidium iodide staining and flow cytometry. (B) Nutlin induces comparable CDKN1A/p21 mRNA levels in proliferating cells, thymidine-arrested cells, and during $S$ phase. U2OS cells were treated as in A or grown asynchronously. Subsequently, CDKN1A/p21 mRNA (RT-PCR) and protein (cf. Suppl. Fig. V.I.7.1A; immunoblot analysis) levels were determined in triplicate $(n=2)$. 
To broaden this analysis, we performed next generation RNA sequencing to identify Nutlin-inducible genes in thymidine-blocked vs. released cells. The induction of most p53responsive genes was largely unchanged regardless of the thymidine block. Less than ten genes were no longer found induced by Nutlin when the cells were allowed to proceed in S phase (Fig.V.I.1C, Suppl. Fig.V.I.7.1B, Suppl. Table 1).

Thus, most capabilities of p53 to activate transcription are preserved while cells replicate their DNA. Previous investigations have mostly used hydroxyurea to arrest cells in $S$ phase. Then, the expression of p53-responsive genes was indeed found attenuated (Gottifredi et al., 2001; Mattia et al., 2007). We propose that unperturbed S phase, but not an intra S phase arrest, permit full p53 activity.

C
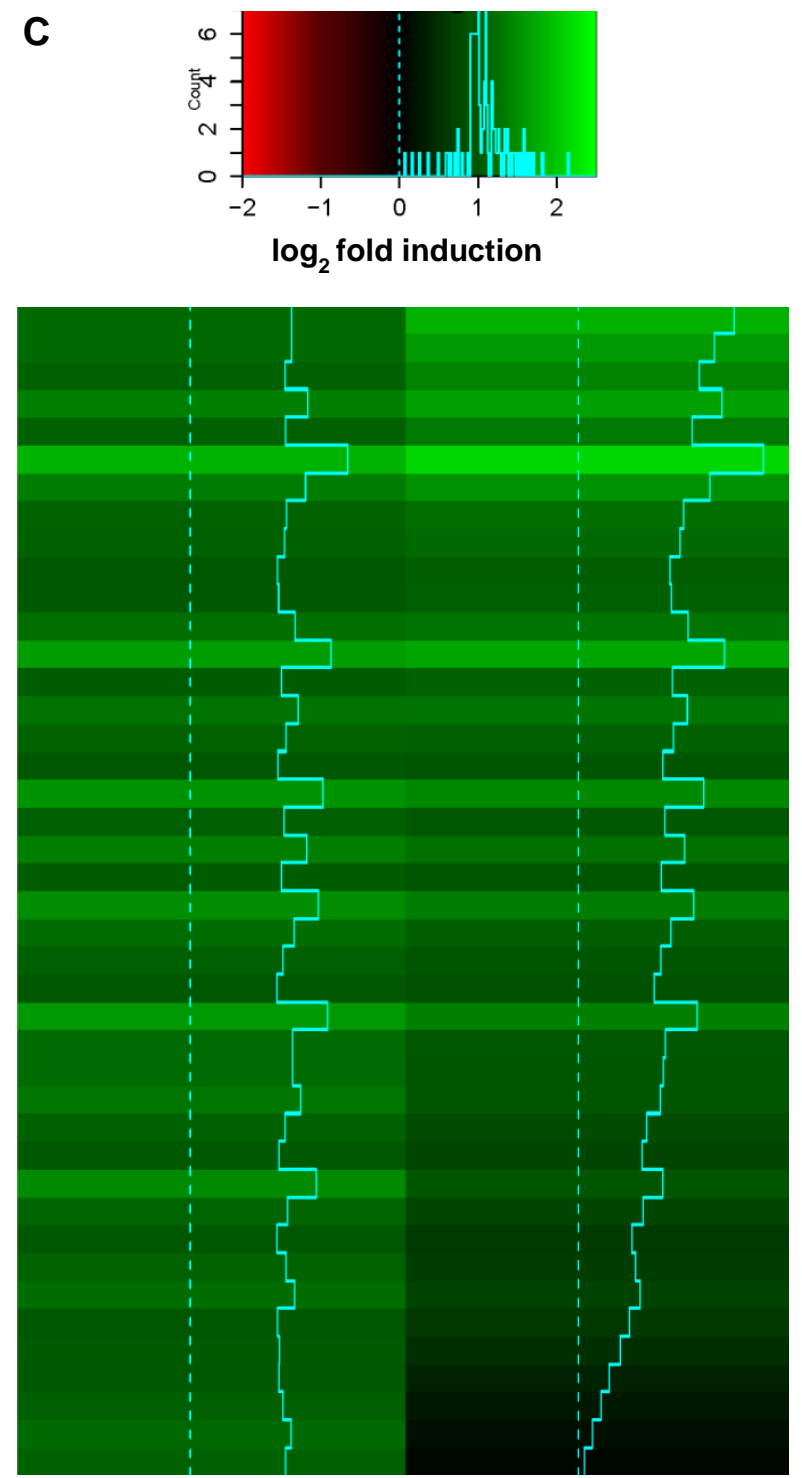

non-released
S-phase
Fig. V.I.1 p53-mediated induction of genes during $S$ phase.

(C) RNA deep sequencing analysis reveals comparable induction of genes by Nutlin, in thymidine-block as well as during $S$ phase. U2OS cells were treated as in $\mathbf{A}$, followed by reverse transcription and next generation sequencing (Illumina). The heat map reflects fold induction of the indicated genes by Nutlin according to the color scheme (color and blue line, log 2). Genes displaying an induction of greater than 2 -fold and a p-value $<0.05$ (based on at least two independent samples for each condition) in thymidine-blocked cells (Suppl. Table S1) were included in the analysis. Most of these genes were induced by p53 to a similar degree when the cells had been released to enter $S$ phase. The genes were sorted according to their $p$-value identified in released cells. Only a small proportion of genes (bottom of the heat map) were inducible only in nonreleased cells but not in $\mathrm{S}$ phase. 


\section{V.I.3.2 p53 activation enhances DNA replication processivity}

Next, we asked whether p53 might exert a genome-protective function during $\mathrm{S}$ phase by affecting DNA replication. U2OS cells were first treated by Nutlin to induce p53 activity, as confirmed by accumulation of $\mathrm{p} 53$ and its target gene products (Fig.V.I.2A).

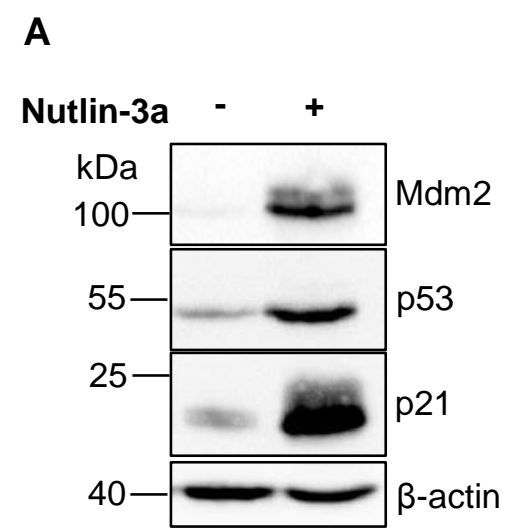

\section{Fig.V.I.2 Enhanced replication fork progression upon p53 activation.}

(A) U2OS cells were treated with $8 \mu \mathrm{M}$ Nutlin or the DMSO solvent for $14 \mathrm{hrs}$ and subjected to immunoblot analysis, detecting Mdm2, p53, $\mathrm{p} 21$, and $\beta$-actin to reveal the induction of p53 and its target gene products. A representative result of three biological replicates is shown.

Subsequently, the characteristics of DNA replication were assessed by DNA fiber assays. The cells were sequentially incubated with two different nucleoside analogues. Upon spreading of the DNA on glass slides, we determined the length of DNA tracks that were detected by antibodies due to incorporation of the labels (Fig.V.I.2B). Strikingly, the replication fork rate, indicating the distance that a replication fork moves within a given amount of time, consistently increased when the cells had been treated with Nutlin before adding the labelling nucleosides (Fig.V.I.2C-D). Of note, increased fork rate was only observed when Nutlin treatment was long enough to fully induce its target genes p21 and Mdm2, while DNA replication still continued (Suppl. Fig.V.I.7.2A-F). We cannot exclude, however, that Nutlin may have shifted a majority of DNA-replicating cells towards the late $S$ phase. Shortening the incubation time with the second label IdU still allowed the observation of an increased fork rate upon Nutlin treatment (Suppl. Fig.V.I.7.2G-I). Conversely, the rate of origin firing, as determined by a stretch of first label flanked by two stretches of second label, was reduced upon Nutlin treatment (Fig.V.I.2E). This is in agreement with the frequent observation that replication fork progression rate and origin firing are inversely correlated (Petermann and Helleday, 2010). However, interfering with origin firing by an inhibitor of Cdc7, as described previously (Montagnoli et al., 2008), did not compromise the increased fork progression rate in the presence of Nutlin, whereas Cdc7 inhibition was itself sufficient to induce p53 and to enhance the fork rate (Suppl. Fig.V.I.7.3A-C). Thus, the increased fork rate by p53 does not strongly depend on origin firing. Counterstaining of the non-labeled fibers confirmed the notion that the labeled track 
is part of a larger, intact DNA fiber (Suppl. Fig.V.I.7.3D). Taken together, the activation of p53 enhances the ability of replication forks to move along the template DNA.

B

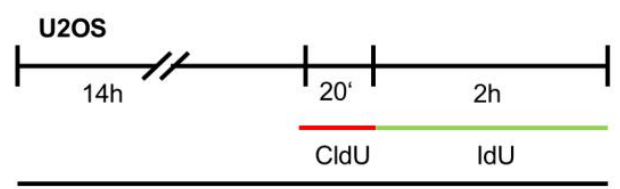

Nutlin-3a

D

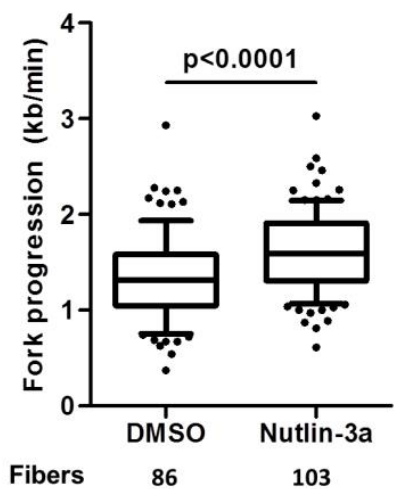

C

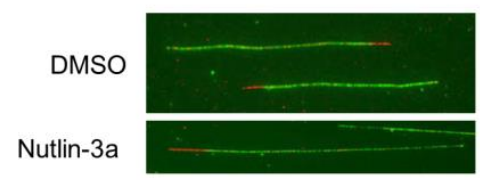

E

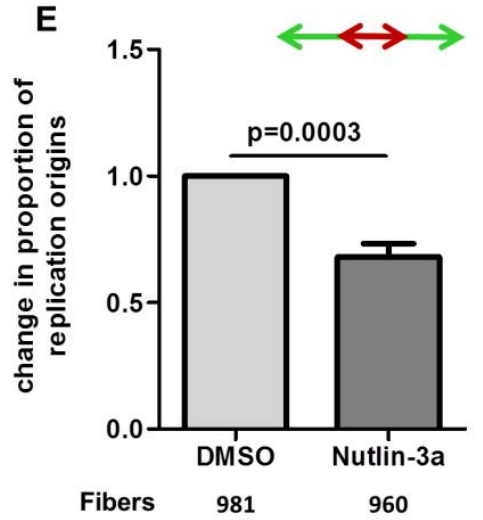

Fig.V.I 2 Enhanced replication fork progression upon p53 activation.

(B) U2OS cells were treated as described in A and further incubated with 5-Chloro-3'deoxy-uridine (CldU, 25 $\mu \mathrm{M}, 20 \mathrm{mins}$ ) followed by lodo-deoxy-uridine (IdU, $250 \mu \mathrm{M}, 120 \mathrm{mins}$ ) as indicated, to label newly synthesized DNA. (C) Tracks of newly synthesized DNA were visualized by immunostaining of CldU (red) and IdU (green). (D) Fork progression was determined through the length of the second label (IdU; kilobases/min). Nutlin pretreatment caused a highly significant increase in the fork rate. Note that fork progression is not identical to fork velocity, since shortening the observed track length can be a result of either slower replication or otherwise of sudden fork stalling. In addition to the experiment shown here, two replicates are shown in Suppl. Fig. 2D. (E) The proportion of first pulse origins, characterized by 1st label in the middle flanked by 2nd label at both sides, among all labelled structures was determined. Nutlin-pretreated cells had a lower proportion of firing origins, in agreement with lower replicative stress. The number of evaluated fibers with labelled tracks from five independent experiments is indicated below each box.

In principle, two phenomena can affect the movement of a replication fork. Either, the speed by which the polymerases move along the template might vary. Otherwise, however, the frequency by which replication stalls entirely can also affect the outcome of a fiber assay when stalling occurs during the period of DNA labeling (Suppl. Fig.V.I.7.3E). To distinguish between these two possibilities, we modified the fiber assay. We incubated the cells with two different nucleosides, with repeated changes every 15 min (Fig. V.I.7.2F-G). In this way, we distinguished the reasons for increased track length upon p53 activation. Longer "stripes" containing one label would indicate faster polymerization, 
whereas a higher rate of fibers that contain all stripes with the two colors would indicate less fork stalling, i. e. higher processivity. Upon induction of p53 activity by Nutlin, we found a higher percentage of fibers with the maximum number of labels (Fig. VI.2H-l; Suppl. Fig.V.I.7. 3F; Suppl. Tables 2 and 3). In contrast, the length of the individual labels 2-5 did not significantly differ between the treatments with Nutlin or DMSO alone (Fig.V.I. 2J; Suppl. Tables 2 and 3). Thus, activation of p53 reduces the frequency of fork stalling and increases the processivity of DNA replication.

$\mathbf{F}$

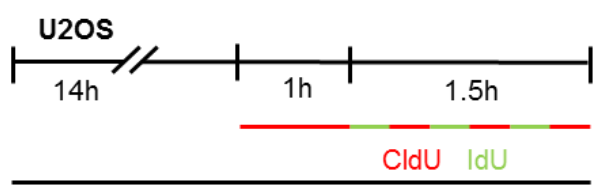

H

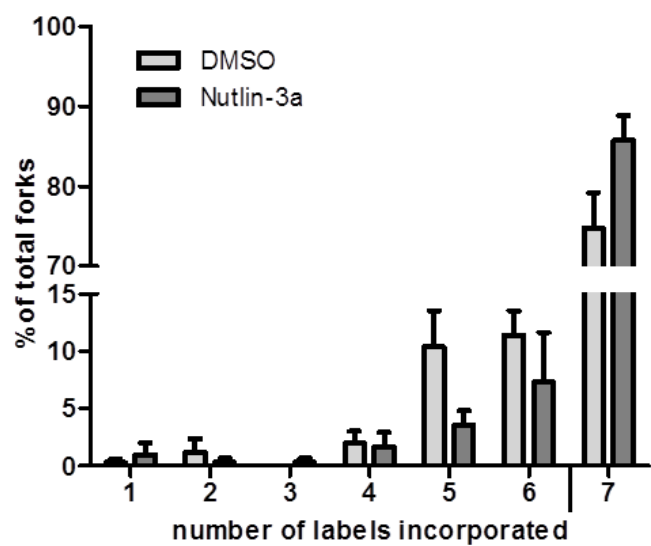

G

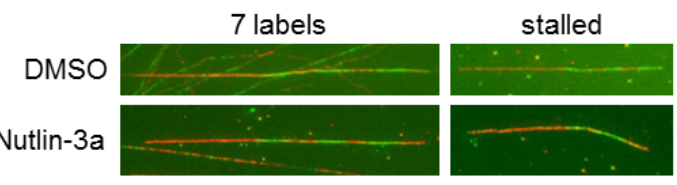

1

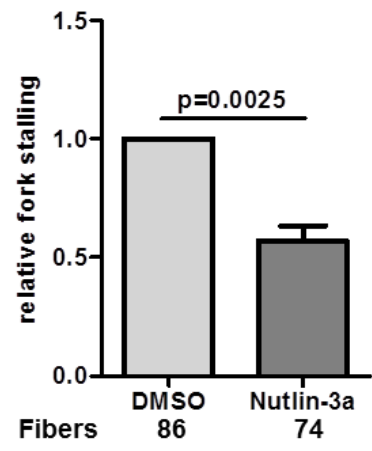

$\mathbf{J}$

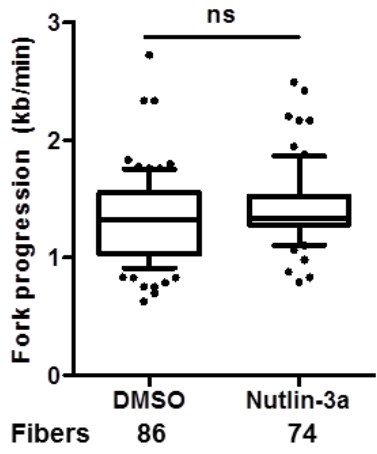

Fig.V.I 2 Enhanced replication fork progression upon p53 activation.

(F) Fiber assay to distinguish the speed of fork progression from the frequency of fork discontinuation (processivity; cf. Suppl. Fig.V.I.7.3E). After treatment as in A, the cells were subjected to one long first CldU label and then repeatedly incubated with IdU and CldU for 15 min each. (G) As a result of fiber staining, a long red track is followed by "stripes" that reflect CldU and IdU incorporation during the subsequent 15 min pulses. Stalled forks are reflected by fibers with less than 7 labels. $(\mathbf{H})$ The number of forks that proceeded through $n$ labels is displayed for DMSO-treated and Nutlin-treated cells. 7 labels reflect full progression of the fork throughout the entire labeling time. Numbers lower than 7 indicate that the replication fork had discontinued during the labeling time. (I) The proportion of prematurely stalled forks (less than 7 labels) indicates that Nutlin-treatment reduced fork stalling and enhanced the processivity of DNA replication. Results from three independent experiments are summarized by the mean and the standard error of the mean. In each experiment, the rate of prematurely stalled forks was determined for DMSO-treated or Nutlin-treated cells, and the relative decrease in this rate upon Nutlin treatment was calculated. (J) Fork speed was determined through the length of labels two to five (kilobases/min). Nutlin treatment did not change the fork speed significantly, in contrast to its impact on fork processivity $(\mathrm{H}, \mathrm{I})$. 


\section{V.I.3.3 p53 depletion hampers replication fork progression, and so does Mdm2 depletion in a p53-deficient background}

We then sought to determine whether the depletion of p53 might also modulate the ability of cells to replicate their DNA. First, we depleted p53 from U2OS cells by siRNA. One set of cells was also treated with gemcitabine, a nucleoside analogue that induces replicative stress by false incorporation and by inhibition of ribonucleotide reductase. To assess the DNA damage response, we determined the levels of phospho- $\mathrm{H} 2 \mathrm{AX}$ by immunoblot analysis. As expected, gemcitabine induced the accumulation of phospho- $\mathrm{H} 2 \mathrm{AX}$ and also increased the levels of p53. Strikingly, however, phospho-H2AX was further increased when p53 had been knocked down (Fig.V.I.3A). We conclude that p53 depletion augments the DNA damage response upon treatment with an inducer of replicative stress.

To directly determine the impact of p53 depletion on DNA replication, we performed fiber assays in the presence or absence of gemcitabine (Fig.V.I.3B). In both cases, we observed a significant decrease in replication fork progression (Fig.V.I.3C-D; Suppl. Fig. V.I.7.4A-F). Thus, baseline levels of p53 carry out a protective function against replicative stress in U2OS cells. The depletion of p53 did not detectably alter the cell cycle distribution of U2OS cells (Suppl Fig.V.I.7.3G-H), and in some contrast with a previous report (Sablina et al., 2005), we did not observe changes in the levels of mitochondrial reactive oxygen species (ROS) upon depletion of p53 (Suppl. Fig.V.I.7.4I).

We then determined whether p53 might affect fork regression upon stalling, as has been reported in BRCA1-deficient cells (Chaudhuri et al., 2016). 
A
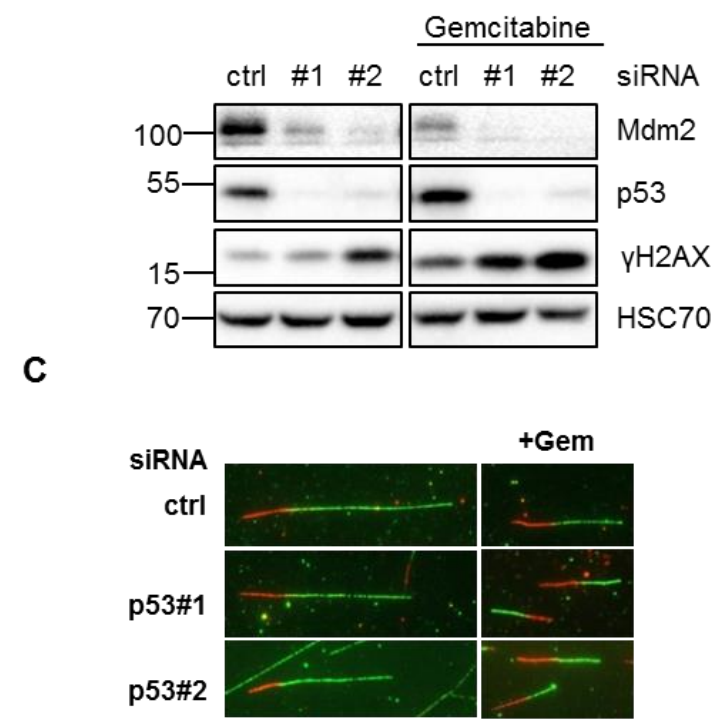

B

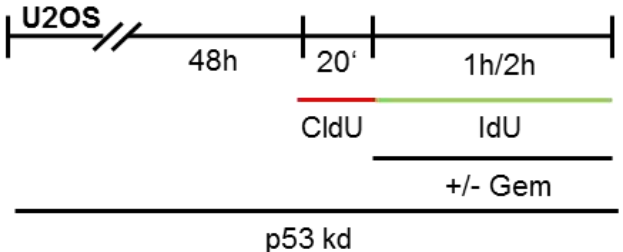

D

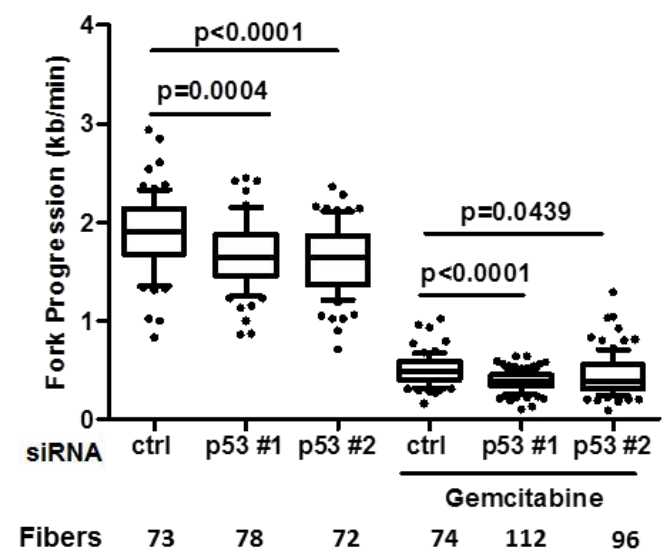

Fig.V.I.3 Increased replicative stress upon p53 depletion, and upon depletion of Mdm2 from p53deficient cell.

(A) U2OS cells were treated with siRNA to knock down p53, or control siRNA, for $48 \mathrm{~h}$, followed by treatment with 400nM gemcitabine for $2 \mathrm{~h}$ and immunoblot analysis. Upon p53 knockdown, decreased amounts of Mdm2 and increased levels of phosphoH2AX $(\mathrm{yH} 2 \mathrm{AX})$ were observed. A representative result out of $>5$ replicates is shown. (B) Upon depletion of p53 (or control siRNA transfection), newly synthesized DNA was labeled with CldU and IdU. Gemcitabine was added during the IdU label only. (C) DNA fibers were immunostained under the conditions described in A and B. (D) Fork progression was determined through the length of the second label (IdU; kilobases/min. p53-depleted cells showed a highly significant reduction in fork progression. This experiment was repeated as shown in Suppl. Fig.V.I.7.4C.

However, the interruption of DNA synthesis by transient treatment with hydroxyurea (HU; Fig.V.I.3E) after the first label did not decrease the proportion of replication forks that proceeded to incorporate the second label, regardless of p53 depletion (Suppl. Fig. V.I.7. $5 A-C$ ), nor did it shorten the track that was labeled before HU addition (Suppl. Fig. V.I.7. $5 D-H)$, as would have been seen in the case of fork regression (Chaudhuri et al., 2016). However, the depletion of p53 decreased the speed of fork progression after release from the HU block (Fig.V.I.3F-G; Suppl. Fig.V.I.7.5I). 
E

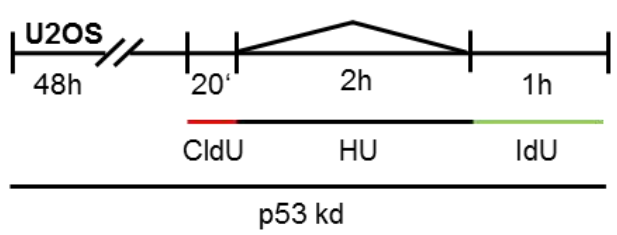

$\mathbf{F}$

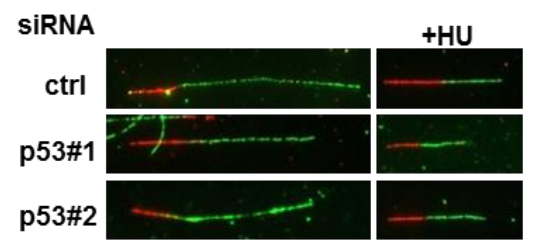

G

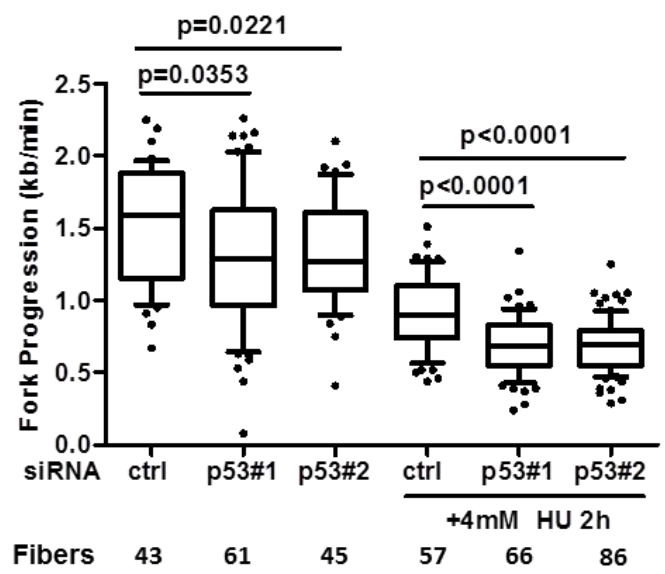

Fig.V.I.3 Increased replicative stress upon p53 depletion, and upon depletion of Mdm2 from p53deficient cells.

(E) U2OS cells were treated with control and p53 siRNA for 48 hours, after which they were labelled with $25 \mu \mathrm{M} \mathrm{CldU}$ for 20 mins. Subsequently, cells were treated with $4 \mathrm{mM}$ hydroxyurea (HU) for $2 \mathrm{~h}$ to arrest replication forks. Upon release from the HU block, the cells were labelled with $25 \mu \mathrm{M}$ IdU for another hour. Control cells were labelled with CldU and IdU without interrupting replication by HU. (F) Labeled replication tracks were immunostained to visualize CldU in red and IdU in green. (G) Fork progression was determined through the track length corresponding to the second label (IdU). P53 depletion significantly reduced the progression of replication forks. This effect was even more pronounced after an HU block. Similar results to this experiment were found independently in Suppl. Fig.5I.

One of the most well-studied p53-responsive genes is Mdm2, encoding a p53 antagonist. Indeed, even the baseline expression levels of Mdm2 were found to depend on p53 (cf. Fig.V.I.3A). We have recently reported an unexpected function of Mdm2 as a chromatin modifier (Wienken et al., 2016), raising the possibility that Mdm2 might affect DNA replication as well. To test this, we depleted Mdm2 from cells and determined the DNA replication fork progression rate. However, knocking down Mdm2 in p53-proficient cells would inevitably have led to robust and long-term activation of p53. Therefore, we subjected a cell line with a targeted deletion of p53 (Bunz et al., 1998) to siRNA transfection, thus knocking down Mdm2 in a p53-deficient background (Suppl. Fig. V.I.7. 5). Strikingly, the depletion of Mdm2 reduced the fork rate of HCT116 p53-/- cells and exacerbated gemcitabine-mediated replicative stress (Fig.V.I.3H-J). The transcription of known replicative stress response genes was not affected by Mdm2 depletion (Suppl. Fig. V.I.7.5K).This suggests that Mdm2 may act as at least one mediator that allows p53 to protect DNA replication, perhaps through its ability to modify histones and chromatin structure (Wienken et al., 2016). 
H

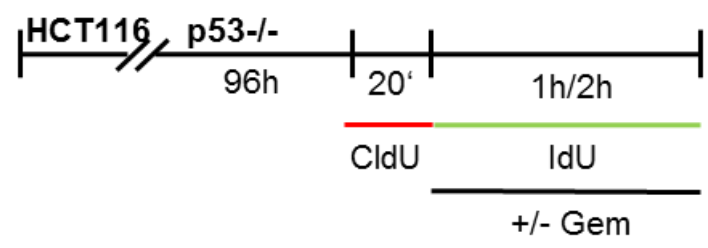

Mdm2 kd

1

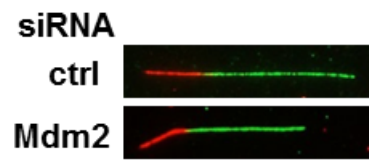

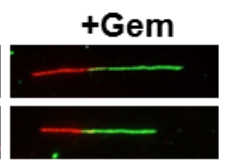

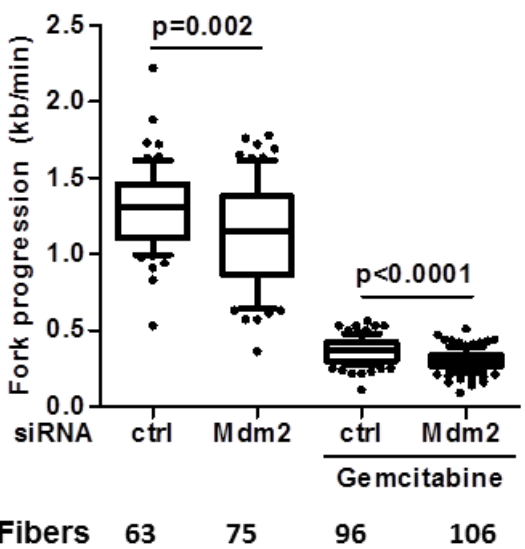

Fibers $\quad 63 \quad 75 \quad 96 \quad 106$

Fig.V.I.3 Increased replicative stress upon p53 depletion, and upon depletion of Mdm2 from p53deficient cells.

(H) HCT116 cells with a targeted deletion of p53 were transfected with siRNA to Mdm2 or control siRNA. Subsequently, fork progression was determined in the presence or absence of gemcitabine. Similar results were obtained with independent siRNAs to Mdm2 (not shown). (I) Representative labeled tracks are shown as in in C and F. (J) Box plot analysis of the fork rates, as determined by the tracks derived from the second label, revealed reduced replication fork progression in response to Mdm2 depletion.

\section{V.I.3.4 p53 promotes the progression of DNA replication forks in murine embryonic fibroblasts}

To validate a possible influence of p53 on DNA replication in non-transformed cells, we employed murine embryonic fibroblasts (MEFs). Wildtype MEFs were treated with Nutlin to induce p53 activity, followed by DNA fiber assays (Fig.V.I.4A). Strikingly, the median DNA replication fork rate was increased 1.5-fold by Nutlin treatment, indicating that p53 is capable of strongly augmenting the movement of DNA replication forks in these cells (Fig.V.I.4B-C).

To test whether baseline p53 levels also affect fork progression, we employed MEFs from mice with a conditional p53 knockout (Jonkers et al., 2001). In these cells, the crerecombinase can be induced by 4-hydroxy-tamoxifen (4-OHT). We were comparing cells that contained wildtype p53 with cells that had had been modified ("floxed") by LoxPinsertions on both p53 alleles, as well as heterozygous cells, in the presence or absence of gemcitabine When cells had been treated with $4-\mathrm{OHT}$ to delete the floxed alleles, we observed that replication fork progression was severely impaired in MEFs with a homozygous p53 deletion (Fig.V.I.4D-F, Suppl. Fig.V.I.7.6A-B). Similarly, the impact of gemcitabine on fork progression was aggravated by loss of p53, even in MEFs where only one allele was lost by recombination (Fig.V.I.4G-I, Suppl. Fig.V.I.7.6C). Some increase in 
the proportion of cells in S phase was also observed in cells that lack one or two p53 alleles (Suppl. Fig.V.I.7.6D-E). In sum, p53 supports DNA replication in primary cells, and for this task, haploinsufficiency of the p53 gene was observed.

A

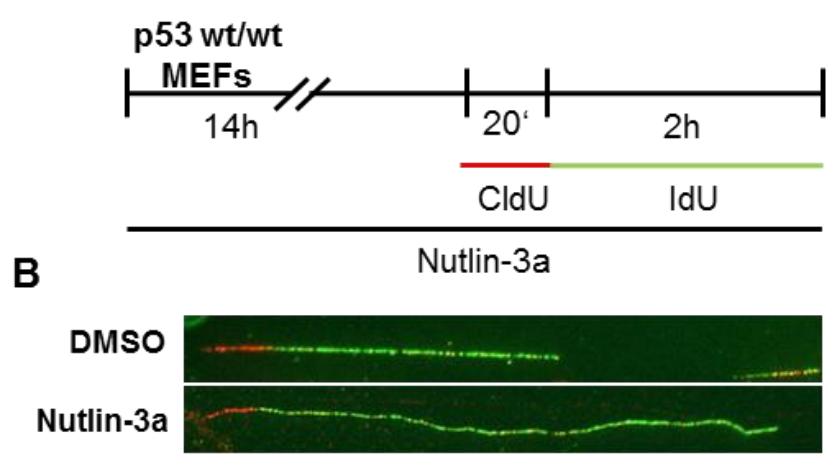

C

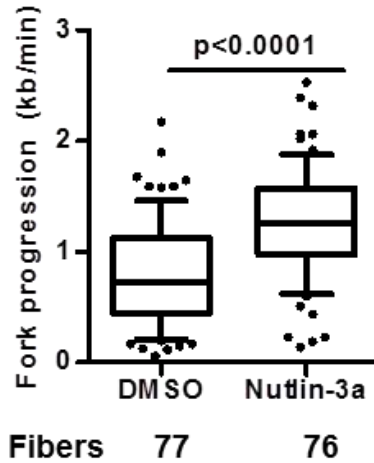

Fig.V.I.4 p53-supported DNA replication in murine embryonic fibroblasts.

(A) Wild type murine embryonic fibroblasts (MEFs) were treated with Nutlin or the DMSO control for 14h, followed by two-label fiber assays as indicated. (B) Fork progression was determined by immunostaining of CldU (red) and IdU (green). (C) The degree of fork progression was increased by Nutlin pretreatment, on a highly significant level. The indicated numbers of labelled fibers were evaluated independently by two independent evaluators. 
D

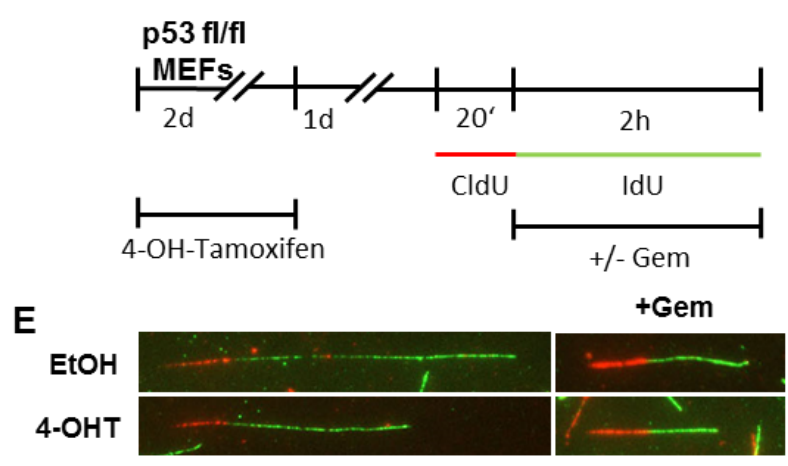

p53 wt/wt, wt/fl, fl/fl

G

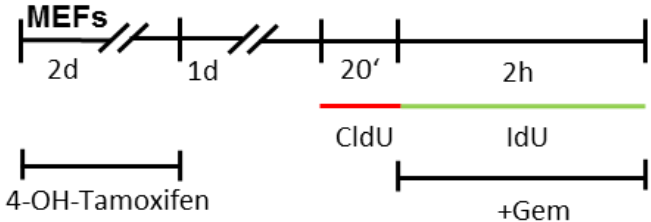

H

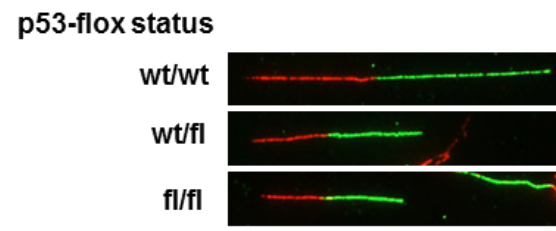

$\mathbf{F}$

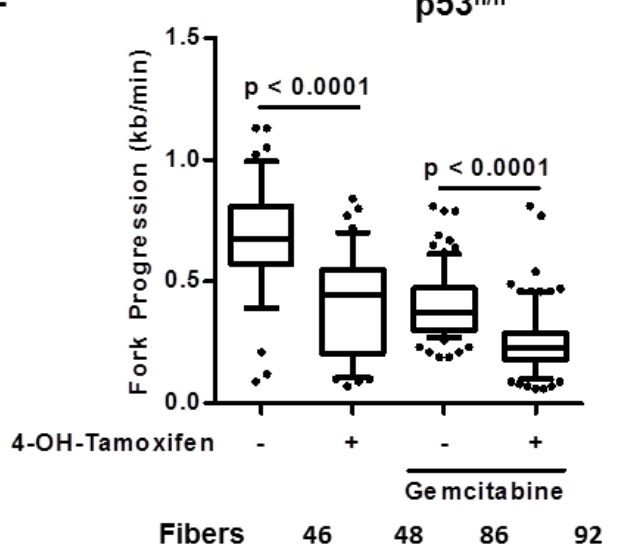

4-OHT + Gemcitabine

I

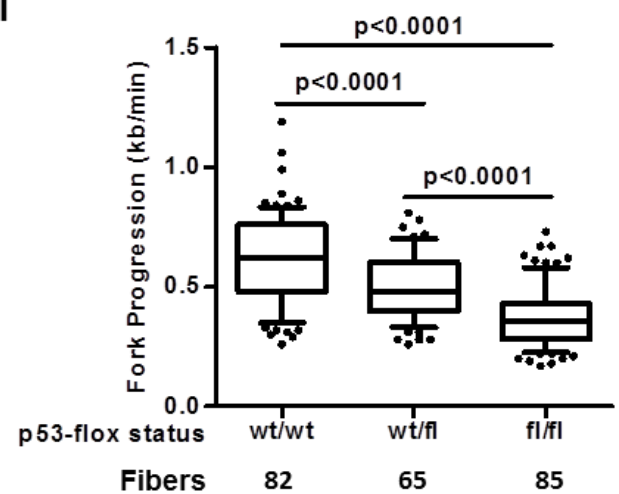

Fig.V.I.4 p53-supported DNA replication in murine embryonic fibroblasts.

(D) MEFs with floxed p53 and a Cre-ERT2 fusion gene driven by the ubiquitously active Rosa26 promoter were first treated with 4-hydroxy-tamoxifen (4-OHT; 200 nM, 48 h) to excise p53 exons 2-10 (Jonkers et al., 2001). Moreover, ethanol was used as a solvent control to 4-OHT. Subsequently, the cells were labelled with

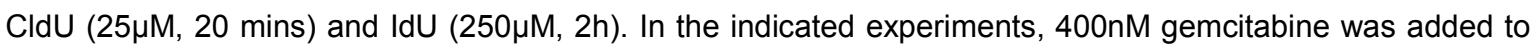
the second label. (E) Labelled DNA tracks were immunostained to detect CldU (red) and IdU (green). (F) When comparing MEFs with two floxed p53 alleles before and after treatment with 4-OHT to excise p53, a strong reduction in fork progression was observed. This was true in otherwise untreated cells and also upon treatment with gemcitabine. A replicate of this experiment is shown in Suppl. Fig.V.I.7.6B. (G) Cells with two wildtype p53 alleles (wt/wt) and with a mixed genotype (wt/fl) were investigated in comparison with the fl/fl p53 genotype. 4-OHT treatment was applied to excise p53 exons 2-10, followed by labelling with CldU $(25 \mu \mathrm{M}$, $20 \mathrm{mins})$ and IdU $(25 \mu \mathrm{M})$, in the presence of gemcitabine, for $2 \mathrm{~h}$. $(\mathrm{H})$ Labelled tracks of newly synthesized DNA were stained with antibodies against CldU and IdU. (I) Labelled track lengths were significantly reduced upon removal of one or both p53 alleles. The result was confirmed in Suppl. Fig.V.I.7.6C.

To assess the role of Mdm2 in DNA replication, we again used a p53-deficient cell system to avoid p53 induction by loss of mdm2. When comparing MEFs with a targeted deletion of p53 alone (single knockout) with cells that lack both p53 and mdm2 (double knockout) (Montes de Oca Luna et al., 1995), we observed a marked decrease in DNA replication 
fork rate in mdm2-deficient cells (Fig.V.I.4J-L). Genes with relevance to replicative stress were not found to be transcribed differentially upon Mdm2 deletion (Suppl. Fig.V.I.7.6F), based on our previous analyses (Wienken et al., 2016). In conclusion, and similar to HCT116 p53-/- cells (cf. Fig.V.I.3H-J), Mdm2 is required for efficient DNA replication fork progression and may thus serve as a mediator allowing p53 to enhance replication processivity.
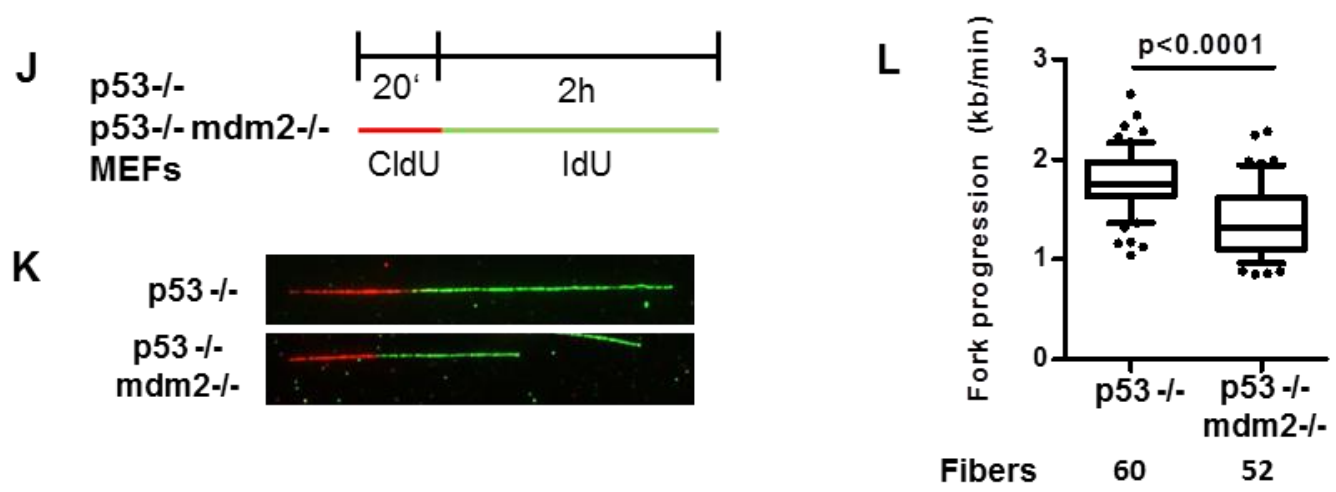

Fig.V.I.4 p53-supported DNA replication in murine embryonic fibroblasts.

(J) MEFs with a p53-/- (single knockout) or a p53-/- mdm2-/- (double knockout) genotype were incubated with the CldU and IdU labels as indicated, followed by analysis of DNA fork progression. Note that these MEFs are not directly comparable to the preparations used in Panels A-I, due to differences in passage numbers and genetic background, as specified in the Materials and Methods. (K) Representative tracks are shown for each genotype. (L) Quantification of fiber lengths revealed that the absence of mdm2, on the p53-/- background, led to a marked loss in replication fork progression.

\section{V.I.3.5 p53 increases DNA replication fork processivity in murine thymocytes before the occurrence of T cell lymphoma}

Finally, we sought to test the impact of p53 on replication fork progression under conditions that are as close as possible to a living organism, and in cells that are prone to cancer formation in animals that lack p53. To this end, we employed thymocytes freshly isolated from p53-/- mice or their p53-proficient littermates, at 4 weeks of age, i. e. at a time point prior to the occurrence of pre-cancerous cell clones (Dudgeon et al., 2014). Thymocytes from p53-/- mice had a higher level of phosphorylated H2AX than the corresponding p53+/+ cells (Fig.V.I.5A), at least compatible with the view that the deletion of p53 leads to enhanced replicative stress in vivo. This was not accompanied by changes in mitochondrial ROS (Suppl. Fig. V.I.7.7A).

Of note, p53-/- thymocytes showed a markedly impaired progression of DNA replication forks, in the presence or absence of gemcitabine, (Fig.V.I.5B-E, Suppl. Fig.V.I.7.7B-G). 
A

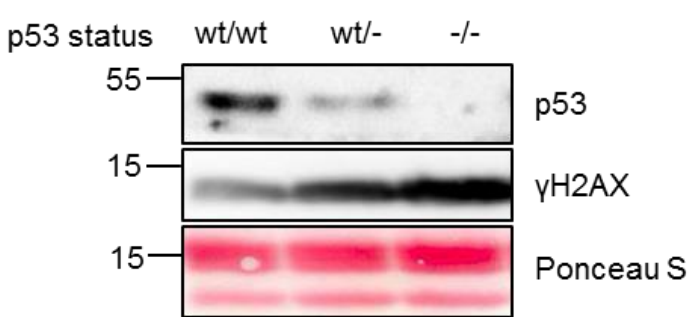

B

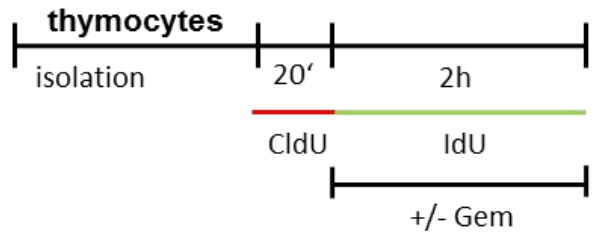

C

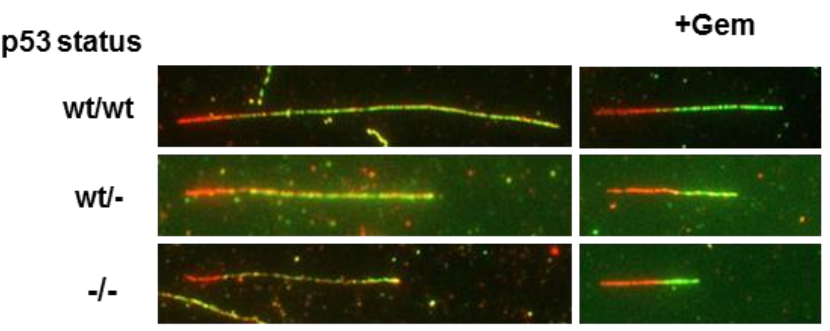

D

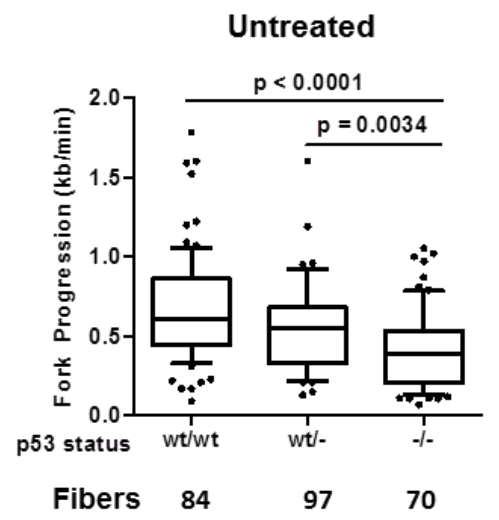

E Gemcitabine

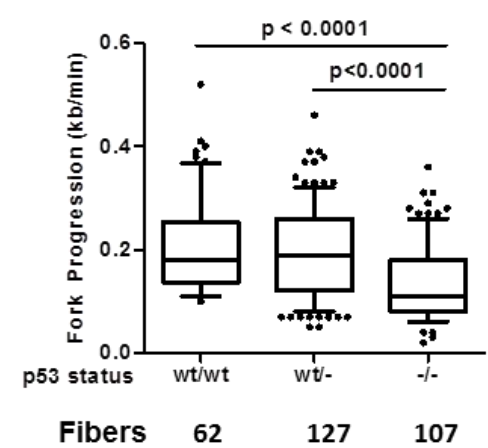

Fig.V.I.5 Replicative stress in murine thymocytes lacking p53.

(A) Immunoblot analysis revealing enhanced H2AX phosphorylation in p53-/- thymocytes. Freshly isolated thymocytes from mice with the indicated genotype were subjected to immunoblot analysis to detect p53 and phospho-Ser 139 Histone 2A ( $\mathrm{YH} 2 \mathrm{AX}$ ). Total protein staining by Ponceau S served as a loading control. (B) Thymocytes were subjected to fiber assays immediately after their isolation from 4 weeks old mice with different p53 status (homozygous knockout, heterozygous, or wild type). The labeling (CldU and IdU, $25 \mu \mathrm{M}$ each) was performed in the presence or absence of $100 \mathrm{nM}$ gemcitabine. (C) Labeled DNA tracks were immunostained to visualize incorporated CldU (red) and IdU (green). (D) Fork progression was assessed by the length of the IdU fibers and displayed by box plots. Decreased fork progression was observed in homozygous knock-out cells, at a highly significant level, in untreated cells, and (E) in gemcitabine-treated thymocytes (confirmation in Suppl. Fig.V.I.7.7C-D).

We also determined the processivity of replication forks by frequent changes in the label (Fig.V.I.5F-J), an assay that we had first performed in U2OS cells (cf. Fig.V.I.2F-J). When p53-/- thymocytes were repeatedly incubated with different labels of newly synthesized DNA, it turned out that the number of stalled replication forks increased in comparison to p53-proficient cells (Fig.V.I.5H, summarized in Fig.V.I.5I). In contrast, the replication speed observed in continuous tracks was not detectably affected by the p53 status (Fig.V.I 5J), nor did we observe gross changes in cell cycle distribution (Suppl. 
Fig.V.I.7.7H-I). Thus, p53 increases the processivity of DNA replication in cells from the thymus. We propose that the resulting replicative stress in response to p53 loss might contribute to the occurrence of chromosomal damage in these cells (Dudgeon et al., 2014), in accordance with the formation of T cell lymphomas, observed later in the lives of more than $90 \%$ of p53-/- mice (Donehower et al., 1992).

$\mathbf{F}$

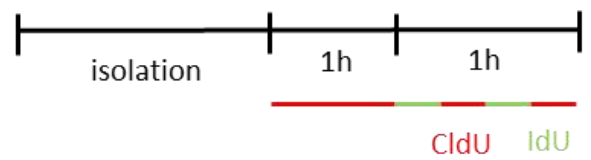

G

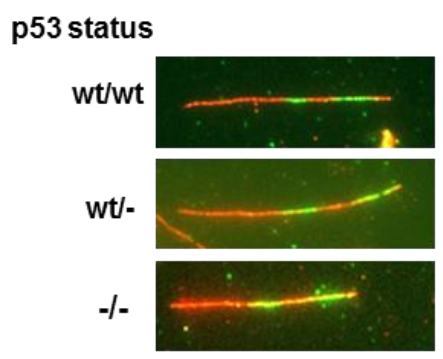

H

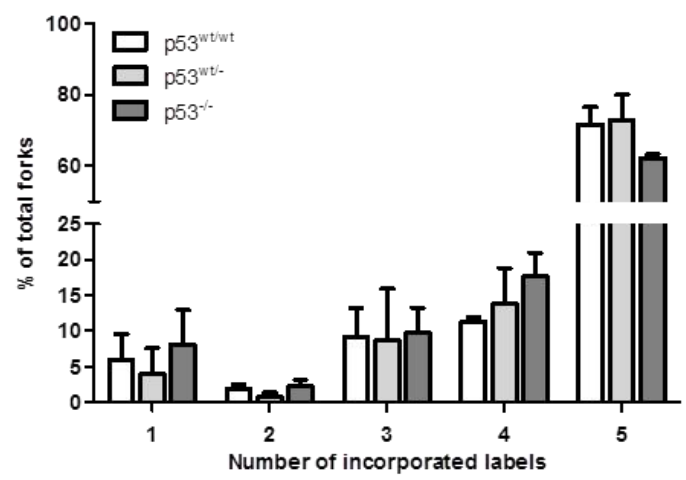

I

Prematurely Stalled Forks

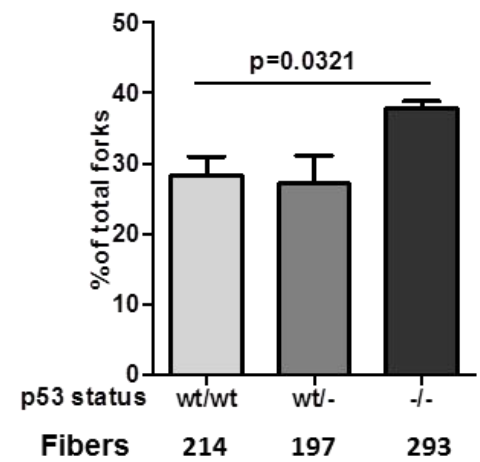

$\mathbf{J}$

Replication Speed Labels 2-4

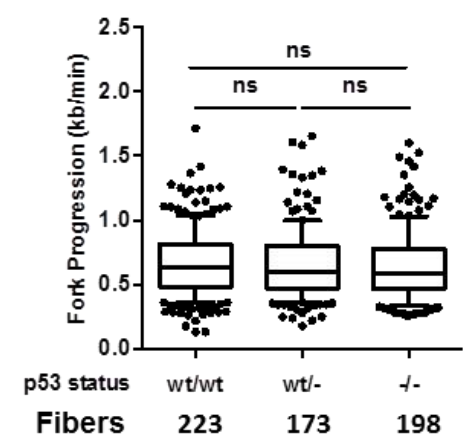

Fig.V.I.5 Replicative stress in murine thymocytes lacking p53.

(F) To determine fork processivity, multiple labels were performed as in Fig.V.I.2F-J, with a total of 5 labels.

(G) Immunostaining allowed the clear distinction of up to five CldU/ldU-labeled tracks. (H) The number of prematurely stalled replication forks (less than 5 labels) was found increased in thymocytes from p53-/- mice, compared to cells from p53 proficient mice. (I) The proportion of prematurely stalled replication forks was significantly increased in p53-deficient thymocytes. Results from three independent experiments are summarized by the mean and the standard error of the mean. In each experiment, the rate of prematurely stalled forks was determined for cells from mice with a p53 +/+, +/- and -/- genotype, and the relative decrease in this rate depending on the deletion of the p53 alleles was calculated. (J) As observed in U2OS cells (Fig.V.I.2J), the replication speeds observed in single tracks, here determined by labels $2-4$, were unchanged. 


\section{V.I.4 Discussion}

P53 is active during $S$ phase, and it strengthens the processivity of DNA replication. This was not only observed in p53-proficient cancer cells, but also in primary cells. Even thymocytes, the precursors of the most frequently found tumor species in p53-/- mice, displayed impaired DNA replication. These observations all support the view that p53 is not only acting ex post, by eliminating or repairing cells that suffered DNA damage. Rather, p53 appears to avoid replicative stress and associated damage ex ante.

Our previous studies had already indicated that p53 activation by Nutlin can protect p53proficient cells from the cytotoxic effects of gemcitabine (Kranz and Dobbelstein, 2006), and also from the combined effects of Wee1 inhibitors and gemcitabine (Li et al., 2015). However, in those experiments, we had pretreated the cells with Nutlin for an extended period of time, largely avoiding their entry into $S$ phase. It appears obvious that cells are resistant to replicative stressors when not in S. Here, however, we show that p53 activity reaches far beyond cell cycle arrest to protect the cell against nucleoside analogues. Even under conditions where cells still undergo DNA replication, p53 still exerts protection against replication fork stalling.

The vast majority of mice that lack p53 will develop T cell lymphoma, at ca. six months of age (Donehower et al., 1992). Deep sequencing analysis revealed that the first clones with rearranged DNA become detectable at nine weeks after birth (Dudgeon et al., 2014). Our results show that replicative stress occurs in the bulk of thymocytes even earlier, i.e. at four weeks of age. This strongly argues that replicative stress precedes the occurrence of detectable premalignant cell clones in p53 -/- mice. It is at least conceivable that replicative stress in thymocytes plays a causal role for tumor formation. In such a scenario, the attenuation of replicative stress would represent an essential part of the tumor suppressive activity of p53. Accordingly, it is noteworthy that even the triple deletion of the p53 target genes cokn1a, pmaip1/noxa and bbc3/puma does not recapitulate the phenotype of p53 null mice. The triple deletions do not give rise to $T$ cell lymphoma or other cancers (Valente et al., 2013). Rather, p53 target genes that support DNA replication, e.g. mdm2, might represent mediators of tumor suppression in vivo.

It would be tempting to speculate that p53 can support DNA replication by physical association with the replication fork. It has long been noticed that p53 is capable of interacting with insertion/deletion mismatches which may form during replication (Lee et al., 1995), and with the "chickenfoot" structure at retracted replication forks (Subramanian and Griffith, 2005) in vitro. However, in our hands, no visible fork regression was found when depleting p53 (Suppl. Fig.V.I.7.5D, H), and the accumulation of p53 only affected 
DNA replication fork progression if it had lasted for half a day (cf. Fig. V.I.2A-D and Suppl. Fig.V.I.7.2A-F), an amount of time required to enhance the mRNA levels corresponding to most p53 target genes (Zhao et al., 2000). While not excluding transcription-independent contributions of p53, e.g. by suppressing excessive homologous recombination (AriasLopez et al., 2006; Bertrand et al., 2004; Janz and Wiesmüller, 2002), we therefore propose that p53-target genes are at least necessary for the impact of p53 on DNA replication. And indeed, one of the genes with the strongest p53-inducibility, Mdm2, was found to facilitate DNA replication independent of its ability to antagonize p53 (Fig. V.I.3 HJ, Fig.V.I.4J-L). Interestingly, Mdm2 acts as a chromatin modifier, as we have shown recently (Wienken et al., 2016), and chromatin modifications are capable of interfering with DNA replication in multiple ways (Alabert and Groth, 2012). However, Mdm2 is also capable of binding Nbs1 (Bouska et al., 2008), raising the possibility that it might support the functions of the MRN complex in resolving replication (Bruhn et al., 2014) or in activating ATR-Chk1-signaling (Duursma et al., 2013). Mdm2's anti-oxidative activity (Riscal et al., 2016) may also contribute to smooth DNA replication, although we have not observed increased ROS upon removal of p53 (Suppl Fig.V.I.7.4I and 7A). Curiously, Mdm2 was also reported to enhance replicative stress when overexpressed (Frum et al., 2014). However, transgenic overexpression of Mdm2 has long been known to yield paradoxical growth arrest rather than its physiological oncogenic function (Brown et al., 1998), and similar effects might be responsible for the observed replication stress in overexpression systems. In our study, we thus avoided Mdm2 transgenes, and all observations were made by exploring Mdm2 from an endogenous source.

Perhaps similarly, a recent report described the deceleration of replication fork progression as a response to p53 activity (Hampp et al., 2016), in seeming contrast with our observations and with another report on a role for p53 in avoiding conflicts of transcription and replication (Yeo et al., 2016). However, the majority of the experiments reported by (Hampp et al., 2016) were carried out using transgenic overexpression of p53, which might well have led to non-physiological situations, or in cell systems other than those described in our study. In contrast, we have performed our assays in the very same cells from p53-deficient animals that give rise to cancer later in life, i. e. thymocytes (Fig.V.I.5). There, endogenous wild type p53 contributed to DNA replication with greater processivity than in p53-deficient thymocytes. We therefore propose that in a cancerrelevant system, p53 acts to protect DNA replication forks against stalling and collapse, thus preserving the genome during this highly vulnerable phase.

On top of Mdm2, additional p53 target genes might be involved in the p53-driven support of DNA replication. Candidates include the ribonucleotide reductase p53R2, capable of increasing the available dNTP pool (Nakano et al., 2000; Tanaka et al., 2000). 
Similarly, the p53-inducible IncRNA NEAT1 dampens replicative stress through paraspeckle formation and ATR activation (Adriaens et al., 2016). On the other hand, the p53-inducible gene product $\mathrm{p} 21 / \mathrm{CDKN} 1 \mathrm{~A}$ was reported to enhance rather than inhibit replicative stress, at least under circumstances that involve UV irradiation (Mansilla et al., 2013) or senescence (Galanos et al., 2016). Thus, the impact of p53 on DNA replication may also depend on the exogenous stressors and the cell-specific set of p53-responsive genes. The relatively long time periods of labeling replicating DNA used in many of our experiments might conceivably have led to the collision of forks, considering average replication origin distances (Conti et al., 2007; Lebofsky and Walter, 2007). However, we rarely observed such collisions, perhaps pointing to different replication origin distances between cell species, and also to the slower progression of replication forks between closely situated origins (Conti et al., 2007).

How could replicative stress lead to cancer? In addition to nucleotide misincorporations, cells with incompletely replicated DNA might enter irregular mitosis, giving rise to chromosomal instability. In agreement, sites of replicative stress often coincide with common fragile sites, i.e. breakpoints of chromosome rearrangements (Glover et al., 2005). The removal of p53 strongly compromises the G2-M checkpoint which normally prevents the onset of mitosis when DNA is damaged or incompletely replicated (Taylor et al., 1999). Thus, p53 may prevent the accumulation of chromosomal rearrangements by avoiding fork stalling and also premature mitosis. In support of this model, the thymocytes in p53 -/- mice indeed accumulate chromosomal rearrangements, and even chromotrypsis, at ages before full-blown T cell lymphomas arise (Dudgeon et al., 2014).

At least in MEFs, even a heterozygous p53 deletion was capable of increasing replicative stress (Fig.V.I.4). This is similar to the situation in patients suffering from Li-Fraumeni syndrome. There, all somatic cells carry one allele with a p53 mutation and another allele with an intact TP53 gene. So far, the occurrence of cancer in these patients was mostly ascribed to a loss of heterozygosity. In contrast, our results suggest that even the heterozygous situation may increase replicative stress at least in some cell species and thus enhance the probability of mutations or chromosomal rearrangements. Thus, a subset of cells in such patients may be cancer-prone even before the intact allele of p53 is lost. About half of all human malignant tumors carry a p53 mutation. However, tumor progression studies such as those on colorectal cancer revealed that, in general, p53 undergoes mutations late during tumor development (Fearon and Vogelstein, 1990). One reason for this phenomenon might be that the presence of p53, through its ability to ensure unperturbed DNA replication, can support the initial survival of cancer cells. Only at late stages, the tumor cell might be able to tolerate the loss of p53 and the concomitant 
replicative stress. It might then take advantage of the enhanced genomic variability and the consequent ability of adaptation, e.g. during invasion and metastasis. Our results also point to a selective vulnerability of p53-deficient cells towards chemotherapeutics that enhance replicative stress. Indeed, enhanced sensitivity of p53 -/- cells towards selected chemotherapeutics was described more than 15 years ago (Bunz et al., 1999). Recently, this phenomenon was ascribed to an ability of p53 to prevent interference between transcription and DNA replication which would otherwise require topoisomerase activity to be resolved (Yeo et al., 2016). Here, we identify p53 as a supporter of DNA replication processivity, in primary cells that undergo tumor formation when p53 is deleted.

P53 has long been known as a guardian of the genome. However, most of these protective functions appeared retroactive from the point of a single cell, as if a guardian killed his injured master after an attack. Here, however, we identify a proactive way of how p53 prevents DNA damage even before it occurs.

\section{V.I.5 Accession Number}

The gene expression data displayed in Fig.V.I.1C, Supplemental Fig.V.I.7.1B and Supplemental Table 1 are accessible at NCBI/GEO with the accession number GSE87668.

\section{V.I.6 Acknowledgements}

We thank Holger Reichardt for helpful advice regarding the isolation of thymocytes. We thank the transcriptome analysis laboratory (TAL) of GZMB for performing RNA seq analyses, Yanping Zhang for MEFs with p53/mdm2 deletions, and Jos Jonkers for p53 ${ }^{\mathrm{fl}}$ mice. This work was supported by the Else Kröner Fresenius Stiftung, the Wilhelm Sander Stiftung, the Deutsche José Carreras Leukämie Stiftung, the Deutsche Krebshilfe, the Deutsche Forschungsgemeinschaft, the Boehringer Ingelheim Fonds (to IK) and the Studienstiftung des deutschen Volkes (to MW, LM and MF). IK was a member of the Göttingen Graduate School GGNB and of the IMPRS/MSc./PhD program Molecular Biology at Göttingen during this work. 


\section{V.I.7 Supplemental Figures}

A

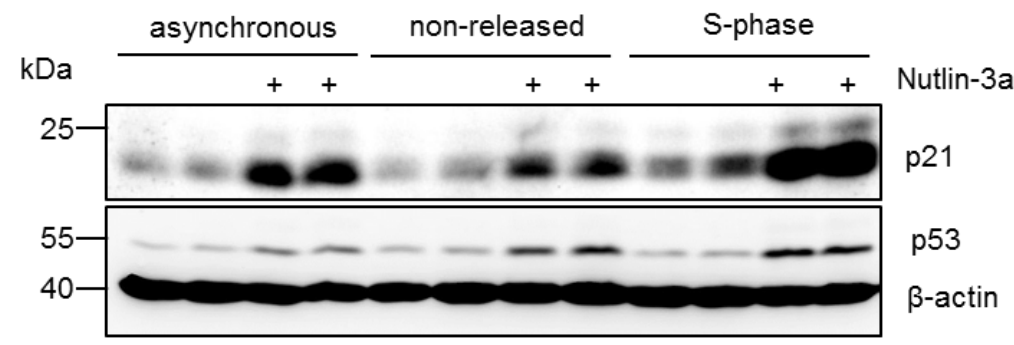

Suppl. Fig. V.I.7.1 p53responsive gene expression during $\mathbf{S}$ phase. Related to Fig. V.I.1

B
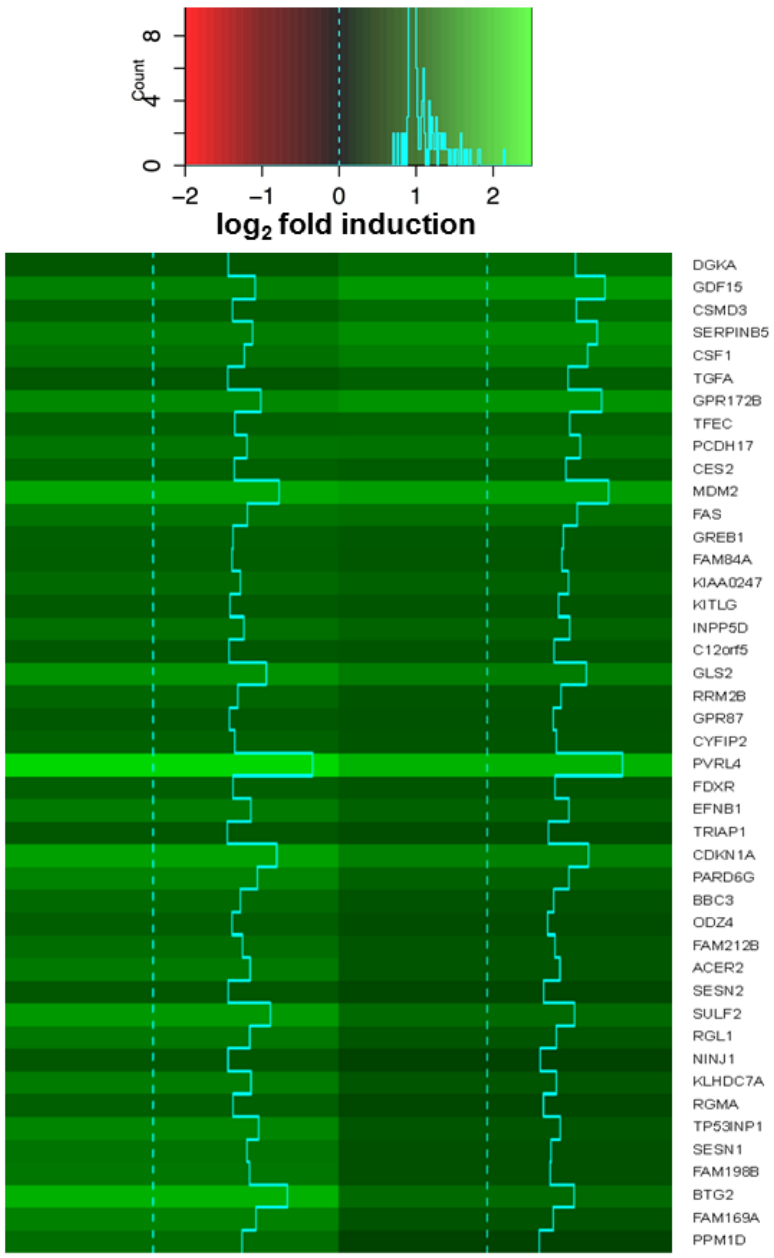

(A) U2OS cells were synchronized by a double thymidine block as described in the legend to Fig. 1, or left asynchronous. Four hours before release from the block, all three sets of cells (S-phase, non-released and asynchronous) were treated with $10 \mu \mathrm{M}$ Nutlin-3a (and the DMSO solvent alone as a control). After the release, the previous concentrations of Nutlin was maintained. The cells were harvested $4 \mathrm{~h}$ after the release from the thymidine block. Immunoblot analysis was performed to detect p53 and its target gene product p21/CDKN1A, as well as $\beta$ actin as a loading control. Samples were processed in duplicate to ensure the reproducibility of the detection levels.

S-phase

non-released

(B) Upon treatment as in A and RNA deep sequencing analysis, genes displaying an induction of greater than 2 -fold and a $\mathrm{p}$-value $<0.05$ in thymidine-blocked and subsequently released cells (Suppl. Table S1) were included in the analysis (left panel). Most of these genes were induced by p53 to a similar degree when the cells had not been released to enter $S$-phase (right panel). The genes were sorted according to their $p$-value identified in arrested, non-released cells. As a result, all genes found to be inducible during $S$ phase were also induced in arrested cells. 
A

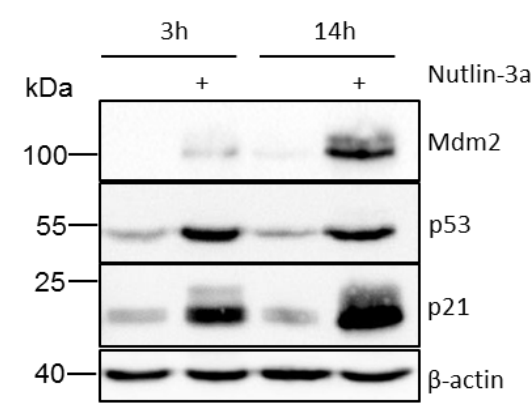

C

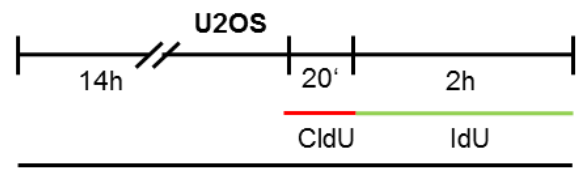

Nutlin-3a

D

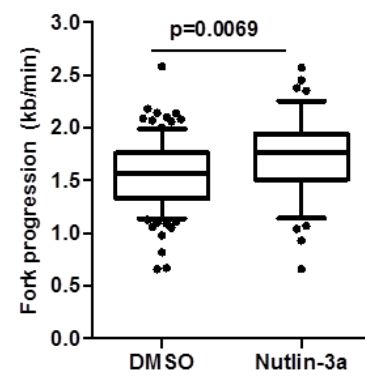

Fibers 108

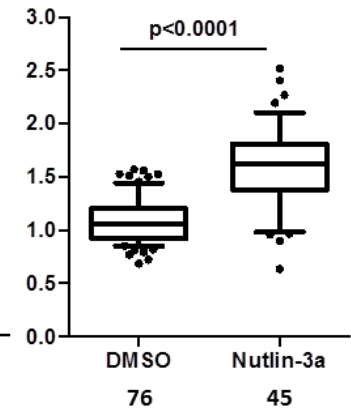

45
B

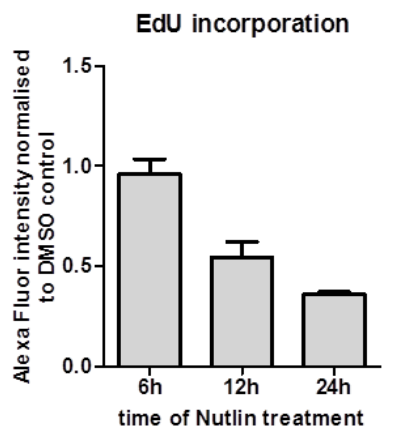

E

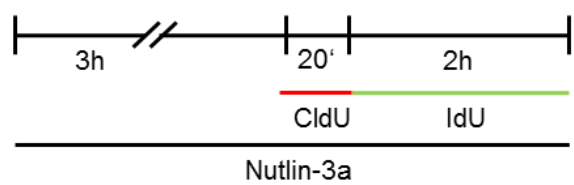

F
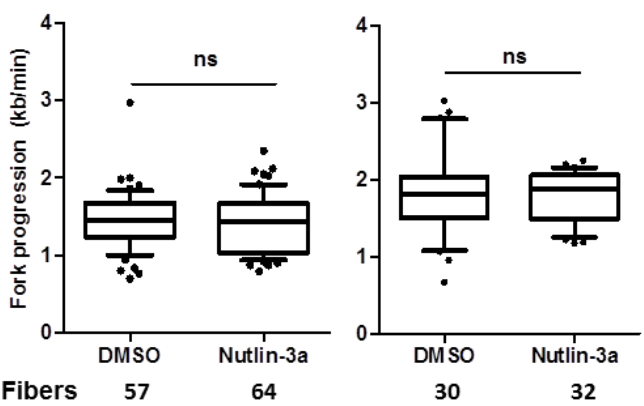

G

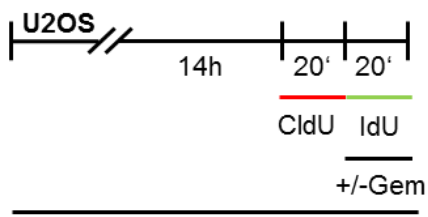

Nutlin-3a

H

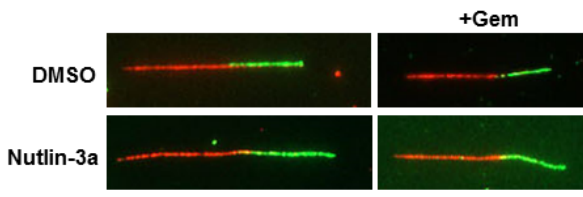

I

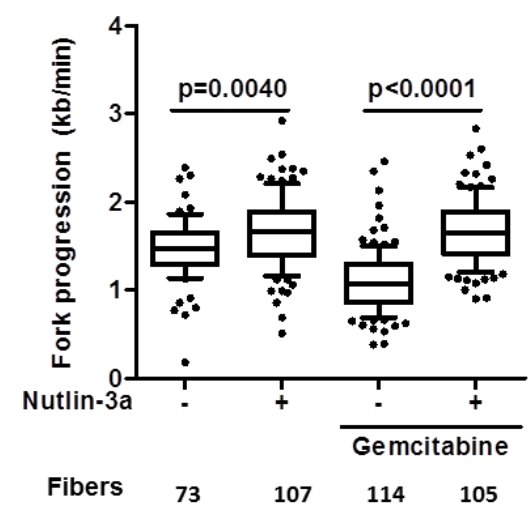

Suppl. Fig. V.I.7.2 Impact of Nutlin on DNA replication in U2OS cells. Related to Fig. V.I.2

(A) U2OS cells were treated with Nutlin for $3 \mathrm{~h}$ or $14 \mathrm{~h}$. Immunoblot analysis revealed comparable p53 accumulation but only less extensive accumulation of p21 and Mdm2 when comparing the 3h incubation with the $14 \mathrm{~h}$ incubation (Blots as in Fig. 2A but including the $3 \mathrm{~h}$ incubation). (B) Nutlin treatment decreases DNA synthesis, but not completely. U2OS cells were treated with Nutlin for the indicated periods of time, followed by incubation with the nucleoside analogue EdU and staining of newly synthesized DNA. The proportion of DNA-synthesizing cells was determined at each time point. (C) Nutlin treatment increases fork progression, as in Fig.V.I.2B-D. U2OS cells were treated with $8 \mu \mathrm{M}$ Nutlin for $14 \mathrm{~h}$, followed by DNA-labelling with $25 \mu \mathrm{M}$ CldU for $20 \mathrm{~min}$, and then $250 \mu \mathrm{M}$ IdU for $2 \mathrm{~h}$. (D) Quantification of fiber lengths from two independent experiments, as in Fig.2D. 
Suppl. Fig. V.I.7.2 continued (E) Three hours of Nutlin treatment do not suffice to increase replication fork progression. U2OS cells were treated with $8 \mu \mathrm{M}$ Nutlin for $3 \mathrm{~h}$, followed by incubation with CldU $(25 \mu \mathrm{M}, 20 \mathrm{mins})$ and IdU $(250 \mu \mathrm{M}, 2 \mathrm{~h})$. (F) The fork rate was not affected by Nutlin treatment for 3 hours. (G) Increased fork rate upon $14 \mathrm{~h}$ Nutlin treatment is still visible by a short (i.e. $20 \mathrm{~min}$ ) labeling protocol. U2OS cells treated with $8 \mu \mathrm{M}$ Nutlin for 14 hours were subjected to short pulses of CldU and IdU (both $25 \mu \mathrm{M}, 20$ mins). Gemcitabine was added to the IdU label as indicated. (H) Immunostaining of CldU (red) and IdU (green) was performed to visualize labeled replication tracks. (I) Nutlin treatment significantly increased fork progression during short labeling times, too, in the presence or absence of gemcitabine. 

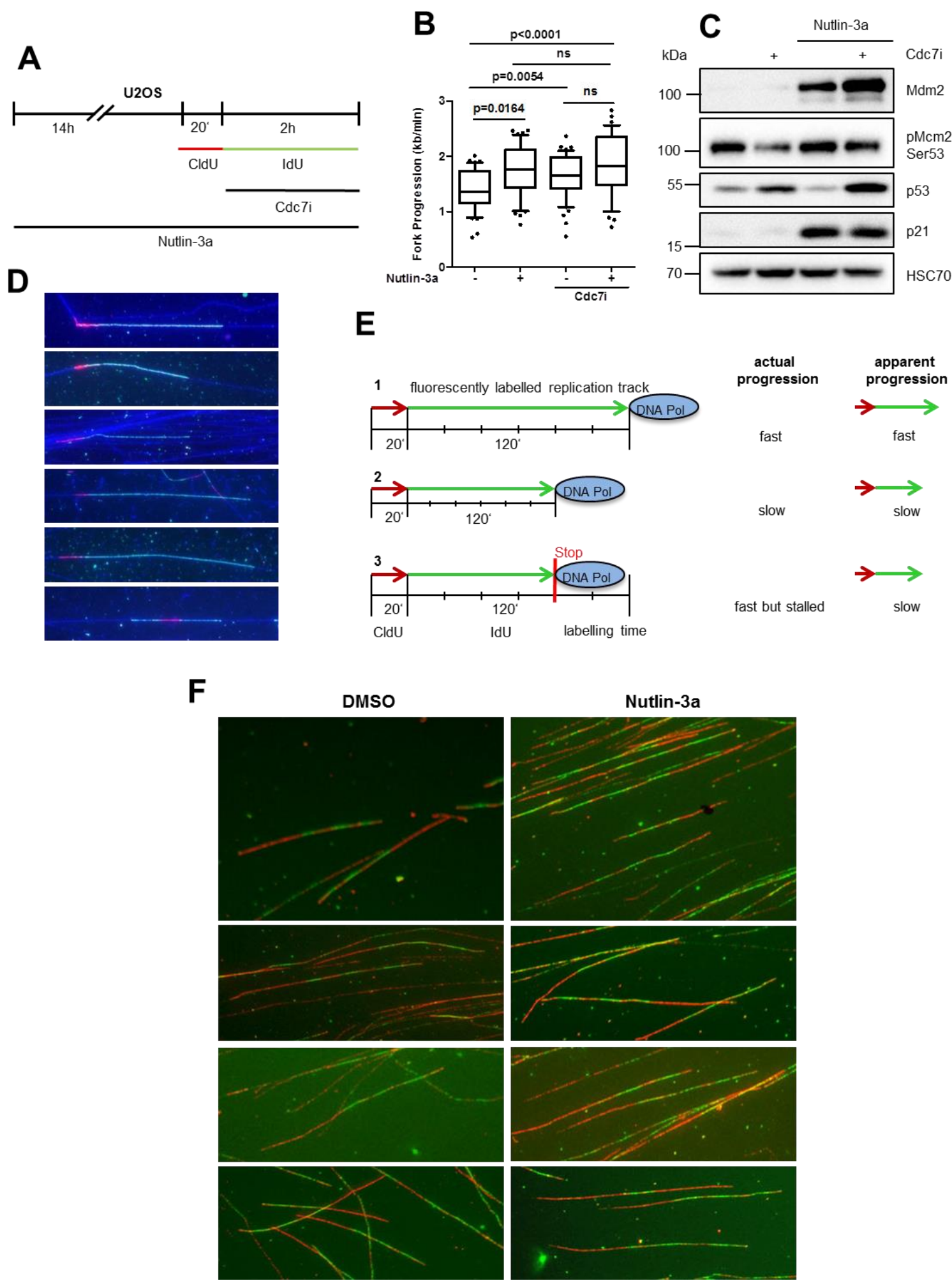

Suppl. Fig. V.I.7.3. Impact of Nutlin on replication processivity in U2OS cells. Related to Fig. V.I.2

(A) Abolishing origin firing by $\mathrm{Cdc} 7$ inhibition does not affect Nutlin-driven fork progression. Fiber assays were carried out with U2OS cells treated with $8 \mu \mathrm{M}$ Nutlin for 14 hours, followed by labelling with CldU $(25 \mu \mathrm{M}, 20$

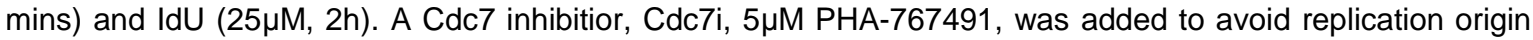
firing (Montagnoli et al., 2008) during the IdU label. (B) As revealed by box plot analysis of the obtained track lengths, Nutlin treatment increased fork progression even in the presence of the Cdc7 inhibitor. Note that the Cdc7 inhibitor alone also increased for progression, in parallel to its ability to enhance p53 levels (cf. Panel C). 
Suppl. Fig. V.I.7.3. continued (C) Immunoblot analysis of samples treated as in Panel B to detect the following proteins: p53 and its target gene products p21 and Mdm2; phosphorylated Mcm2 (Ser53), a bona fide substrate of Cdc7 (Lei et al., 1997); and HSC70 as a loading control. P53 was not only induced by Nutlin, but also by Cdc7 inhibition, in agreement with previously published observations (Montagnoli et al., 2004; Rodriguez-Acebes et al., 2010).

(D) Counterstaining of DNA fibers carrying labelled tracks. After incubating U2OS cells with the labels CldU and IdU, we performed fiber assays as in Fig.V.I.2B-D. Before mounting the fibers, we stained the (denatured) DNA with a third primary antibody, directed against single stranded DNA, followed by a fluorescently labeled (Alexa647) secondary antibody. This made the fibers visible in far red (presented in blue in the composite image) and showed that the labeled tracks were part of a longer DNA fiber. (E) Scheme depicting two different scenarios that could explain a shortened 2 nd label track. When compared to an unperturbed replication fork (1 top), slower DNA fork progression would lead to a shortened fiber (2 middle). However, premature fork stalling within the second labeling period could also lead to shortened fibers, even if the speed of replication forks remains the same (3 bottom). (F) Additional images to document fork processivity and document the feasibility of multiple changes of label, as described in the legend to Fig.V.I.2F-J. For quantification, cf. Suppl. Tables 2 and 3. 
A

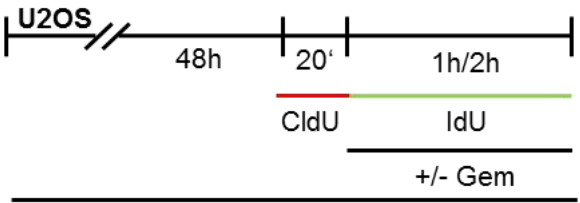

p53 kd

B

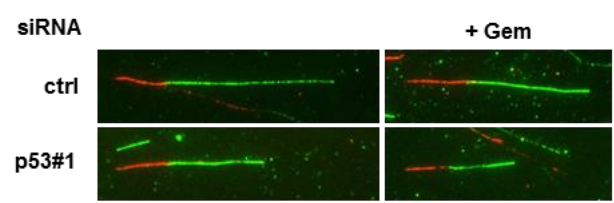

C

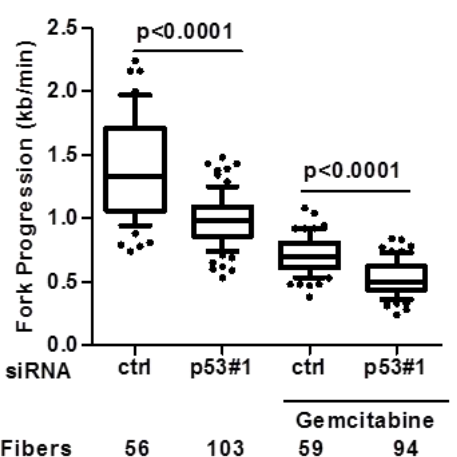

D

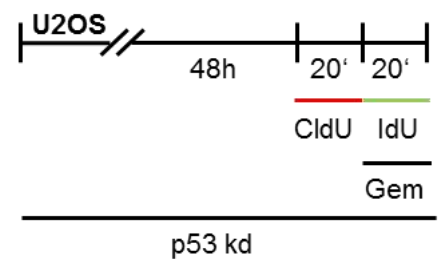

E

siRNA

ctrl

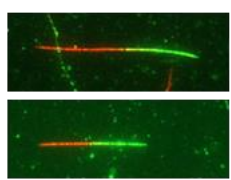

+ Gem

p53\#1

p53\#2

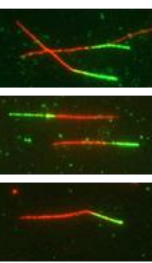

F

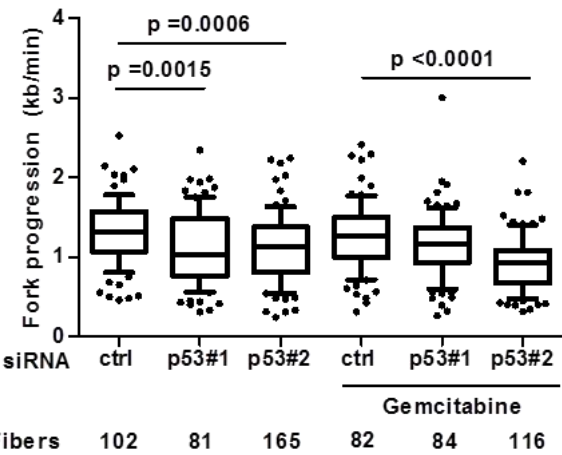

G

ctrl siRNA
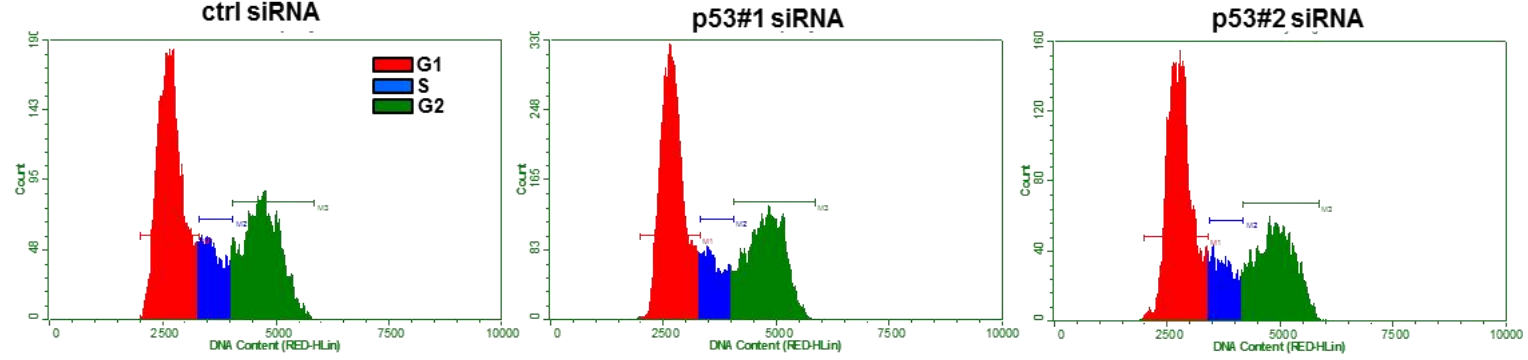

H

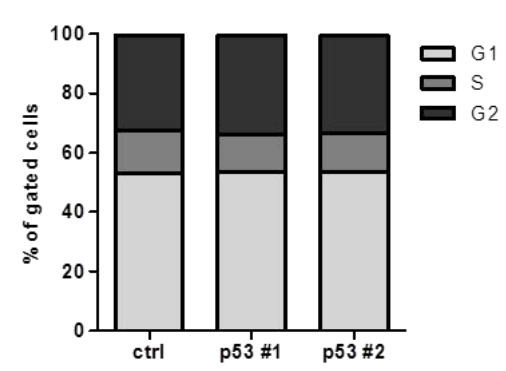

I

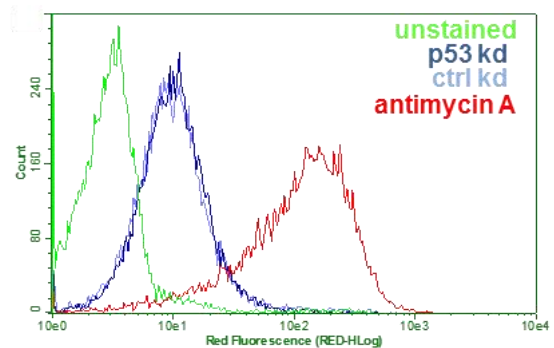

Suppl. Fig. V.I.7.4 Depletion of p53 impairs DNA replication. Related to Fig. V.I.3

(A) To replicate the assays shown in Fig.V.I.3 B-D, U2OS cells were transfected with siRNA to knock down p53, or with control siRNA. Newly synthesized DNA was labeled by $25 \mu \mathrm{M}$ CldU for 20 min and then $250 \mu \mathrm{M}$ IdU supplemented with Gemcitabine for $2 \mathrm{~h}$ and controls for $1 \mathrm{~h}$, followed by fiber assays. (B) Immunostained replication tracks of CldU (red) and IdU (green). (C) The lengths of IdU-labeled tracks were used to assess replication fork progression upon p53 knockdown or control siRNA transfection. In the presence or absence of gemcitabine, p53 depletion yielded reduced fork rates. 
Suppl. Fig. V.I.7.4 continued (D) P53 depletion and fiber assays were carried out as in Panel A, but shorter labeling pulses were applied, i.e. incubations for 20 minutes with CldU and then IdU (both $25 \mu \mathrm{M}$ ). Gemcitabine was added to the second label (IdU) only. (E) Immunostaining revealed short red (CldU) and green (IdU) tracks. (F) The fork rates, as determined by the lengths of the IdU tracks, are displayed as boxplots. Despite the shorter labeling time (and thus reduced likelihood of fork collapse during it), p53dependent differences were still observed. (G) Cell cycle profiles of U2OS cells upon p53 knockdown or control transfection for 48 hours. The cells were fixed, incubated with the DNA-intercalating dye propidium iodide and subjected to flow cytometry. Histograms reflect the proportion of cells within windows of DNA content. (H) The profiles obtained in Panel G were summarized to display the proportion of cells in each phase of the cell cycle. No changes were observed upon depletion of p53. (I) p53 does not detectably affect mitochondrial reactive oxygen levels. Upon transfection with siRNA to p53 vs. control siRNA, U2OS cells were incubated with the dye MitoSox to detect mitochondrial superoxide, followed by flow cytometry. As a positive control, cells were treated with $40 \mu \mathrm{M}$ antimycin A during the incubation with MitoSox. For a negative control, MitoSox was omitted. 
A

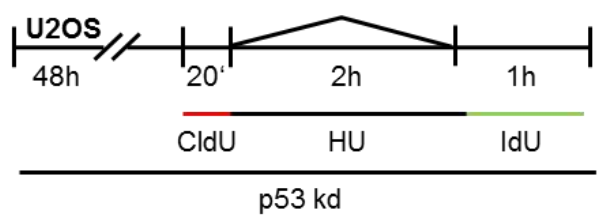

B

B $\quad$ siRNA

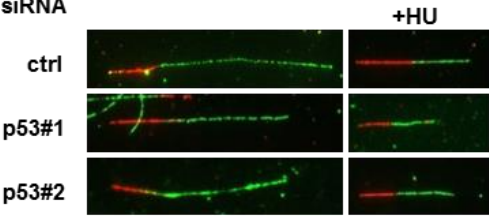

C

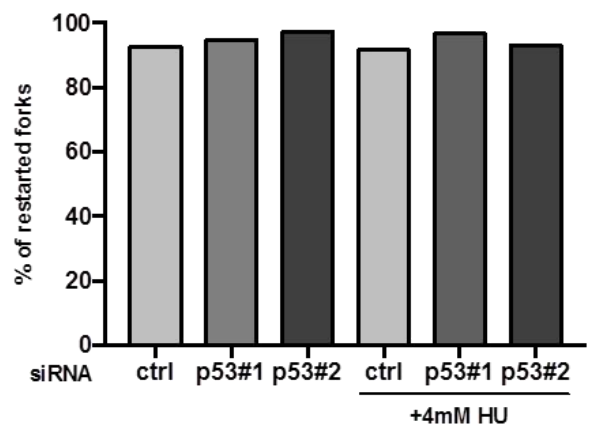

D

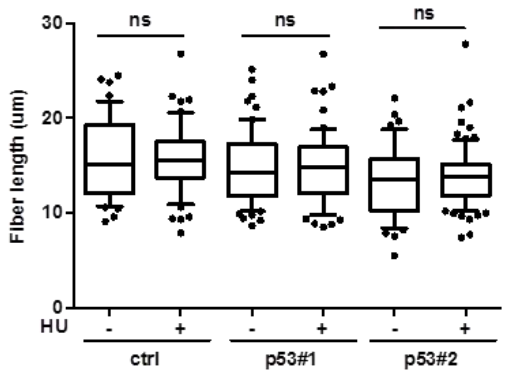

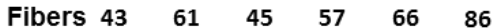

K

\begin{tabular}{|c|c|c|c|c|}
\hline Symbol & Gene Name & $\begin{array}{c}\text { ENTREZ- } \\
\text { ID }\end{array}$ & logFC & p-value \\
\hline ATR & $\begin{array}{c}\text { ataxia telangiectasia and } \\
\text { Rad3 related }\end{array}$ & 545 & -0.01 & $9.64 \mathrm{E}-01$ \\
\hline CHEK1 & $\begin{array}{c}\text { CHK1 checkpoint } \\
\text { homolog (S. pombe) }\end{array}$ & 1111 & 0.11 & $6.31 \mathrm{E}-01$ \\
\hline RAD17 & $\begin{array}{c}\text { RAD17 homolog (S. } \\
\text { pombe) }\end{array}$ & 5884 & 0.02 & $9.04 \mathrm{E}-01$ \\
\hline TOPBP1 & $\begin{array}{c}\text { topoisomerase (DNA) II } \\
\text { binding protein 1 }\end{array}$ & 11073 & 0.06 & $7.66 \mathrm{E}-01$ \\
\hline CLSPN & claspin & 63967 & 0.12 & $6.53 \mathrm{E}-01$ \\
\hline
\end{tabular}

E

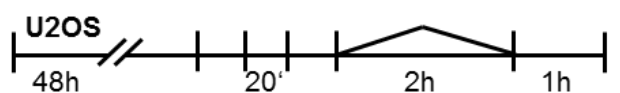

$\mathbf{F}$
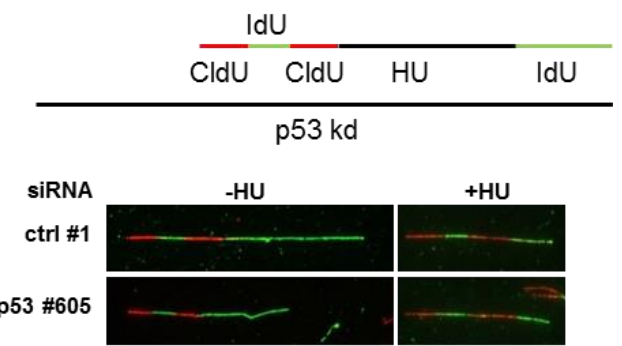

G

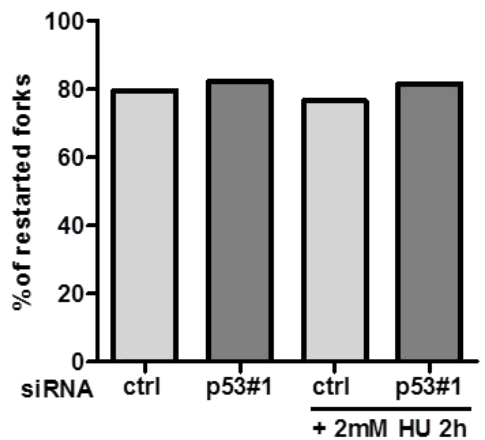

H

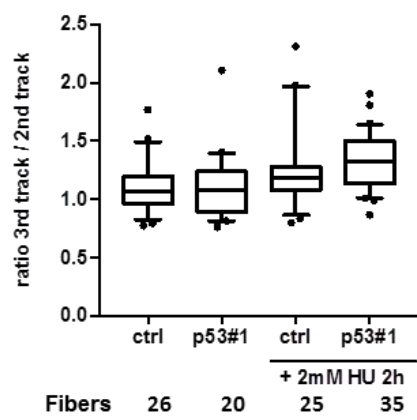

I
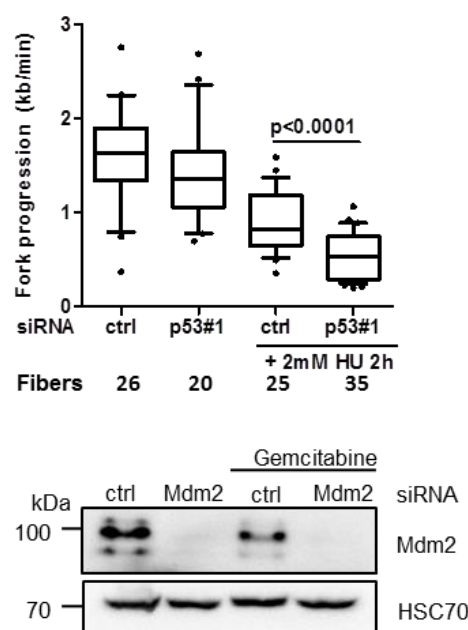

Suppl. Fig. V.I.7.5 Depletion of p53 does not alter fork restart or reversal; Mdm2 mediates fork progression . Related to Fig. V.I.3

(A) The rate of fork restart after transient inhibition of ribonucleotide reductase is not affected by p53 depletion. U2OS cells were transfected to knock down p53 or control-transfected for 48 hours. Labeling with $25 \mu \mathrm{M} \mathrm{CldU}(20 \mathrm{mins})$ and IdU (1h) was interrupted or not by treatment with $4 \mathrm{mM}$ hydroxyurea (HU) for $2 \mathrm{~h}$. 
Suppl. Fig. V.I.7.5 continued (B) Immunostaining of CldU (red) and IdU (green) visualized the continuation of replication after the $\mathrm{HU}$ block. (C) The rate at which forks continue to replicate after the HU block was determined by quantification of red-only (stalled) and red-green (continuing) structures. No difference were observed between the four samples.

(D) Lack of fork regression upon HU treatment. The length of the first label track (before HU block) was determined to reveal any possible fork regression. Regardless of HU treatment or p53 depletion, the length of the first, CldU-labeled track was unaffected. Hence, p53 depletion does not give rise to degradation of stalled replication forks, unlike the depletion of BRCA1 (Chaudhuri et al., 2016). (E) Cells were treated as in Panel A. Instead of one short pulse of CldU prior to HU treatment, the cells were subjected to three short (20 mins) pulses of CldU, IdU, and CldU (all $25 \mu \mathrm{M}$ ) before a $2 \mathrm{~h}$ block with $2 \mathrm{mM} \mathrm{HU}$ and a subsequent $1 \mathrm{~h}$ IdU labeling $(25 \mu \mathrm{M})$. Controls were labeled with IdU for $1 \mathrm{~h}$ directly after the last CldU label. (F) Immunostaining revealed the expected pattern of red (CldU) and green (IdU) labels to indicate that replication continued after the HU block. (G) The rate of fork restart after the $\mathrm{HU}$ label was assessed by quantifying all structures with three short labels only (no restart) and the ones with three short and one long green label (restart). No reduction in the restart rate was seen upon p53 depletion. (H) Lack of fork regression regardless of p53 proficiency. After treatment as in Panel $\mathrm{N}$, the ratio of the $3 \mathrm{rd}$ label $(20 \mathrm{~min} \mathrm{CldU})$ and the 2 nd label (20 min IdU) was determined. As expected, this ratio was close to 1, suggesting that the 3rd track was not subject to fork regression during the $\mathrm{HU}$ block. This ratio was not diminished as a result of p53 knockdown. (I) Replication progression was assessed by measuring the length of the last IdU label (1h), confirming that the preceding HU block impairs the progression of DNA replication, and that p53 depletion further exacerbates replicative stress in this context. (J) Depletion of Mdm2 from HCT116 cells with a targeted deletion of p53. The cells were transfected with siRNA to Mdm2 or control siRNA, followed by immunoblot analysis. The cells were then subjected to fiber assays, as outlined in Fig.V.I.3H-J. (K) RNA levels corresponding to the pathways responding to replicative stress. Upon siRNA-mediated depletion of Mdm2 from p53-deficient HCT116 cells, RNA samples were subjected to deep sequencing analysis. No significant differences in the mRNA levels corresponding to the indicated genes were observed. The data were derived from our previous work (Wienken et al., 2016). logFC, logarithm of gene expression ratio (fold change). 
A

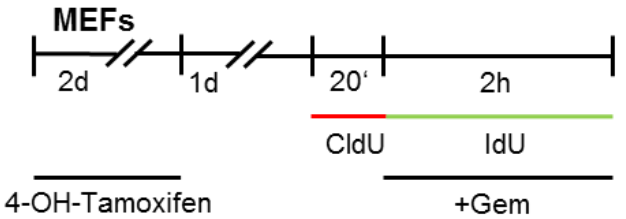

B

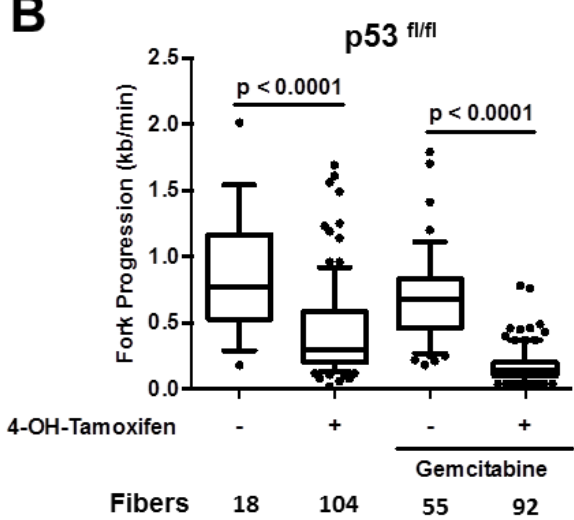

C

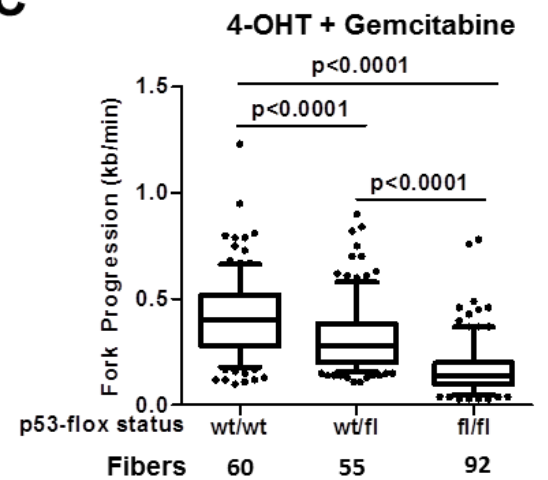

$\mathbf{F}$
D
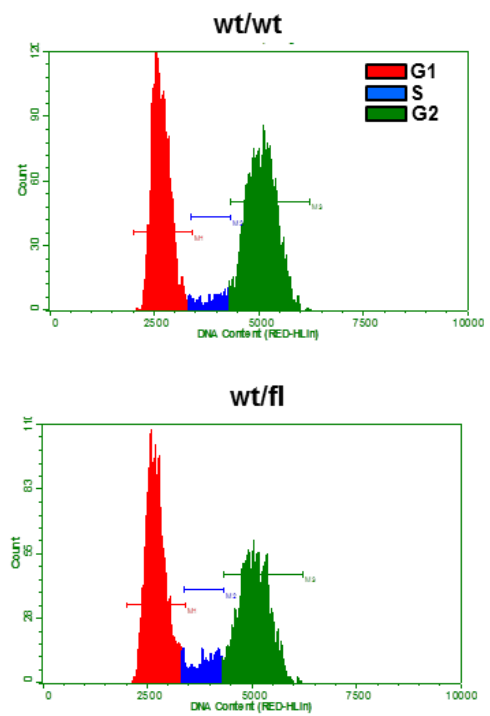

$\mathrm{fl} / \mathrm{fl}$

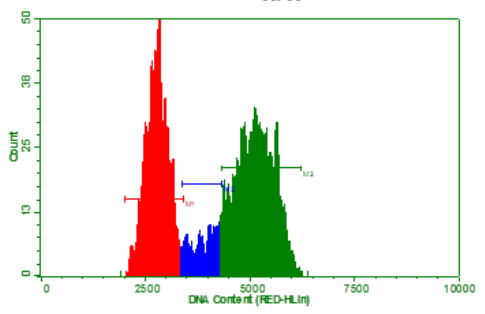

$\mathbf{E}$

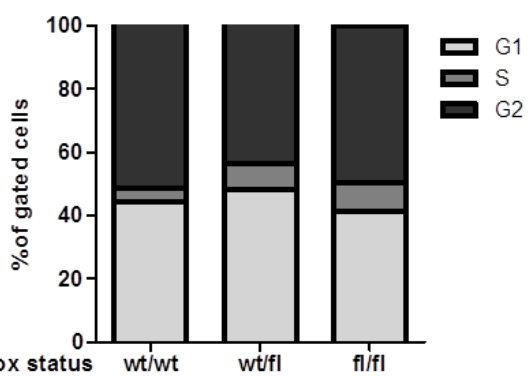

\begin{tabular}{|c|c|c|c|c|}
\hline Symbol & Gene Name & ENTREZ-ID & logFC & p-value \\
\hline ATR & ataxia telangiectasia and Rad3 related & 245000 & -0.10 & 9.77831 \\
\hline CHEK1 & CHK1 checkpoint homolog (S. pombe) & 12649 & 0.33 & 6.43041 \\
\hline RAD17 & RAD17 homolog (S. pombe) & 19356 & 0.05 & 13.3351 \\
\hline TOPBP1 & topoisomerase (DNA) II binding protein 1 & 235559 & 0.03 & 13.8707 \\
\hline CLSPN & claspin & 269582 & 0.19 & 9.84939 \\
\hline
\end{tabular}

Suppl. Fig. V.I.7.6 P53 depletion decreases DNA replication fork progression in murine embryonic fibroblasts (MEFs). Related to Fig. V.I.4

(A) As in Fig.V.I.4D-F, MEFs with ER-cre and floxed p53 were treated with 200nM 4-OHT to remove the floxed p53 gene, or control-treated, for 48 hours. This was followed by incubation with normal media for 24 hours, and subsequent incubation with $25 \mathrm{uM}$ CldU for $20 \mathrm{~min}$, followed by $250 \mathrm{uM}$ IdU for $2 \mathrm{~h}$. During the second labeling period, 400nM Gemcitabine was added as indicated. (B) MEFs with two floxed p53 alleles, treated with 4-OHT and/or gemcitabine, were compared as to their DNA replication, as in Fig. 4F. The removal of p53 by $4-\mathrm{OHT}$ decreased fork progression. 
Suppl. Fig. V.I.7.6 continued (C) Fiber lengths are displayed comparing 4-OHT treated cells with two, one or no floxed p53-allele, treated with gemcitabine, as in Fig. 4l. Even one floxed p53 allele compromised DNA replication, and two floxed alleles did so to a greater extent. (D) The cell cycle distribution of MEFs treated with 4-OHT was determined by propidium iodide and flow cytometry, and summarized in Panel E. No increase in $S$ phase fractions was observed in p53-deficient MEFs. (F) The deletion of mdm2 on a p53-/- MEF background does not detectably alter the mRNA levels corresponding to genes that are relevant to replicative stress. The expression levels were determined by RNA sequencing analysis as in Suppl. Fig.V.I.7 5K, based on the data from (Wienken et al., 2016). 
A

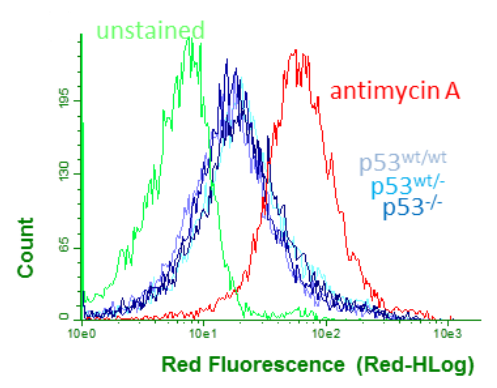

E

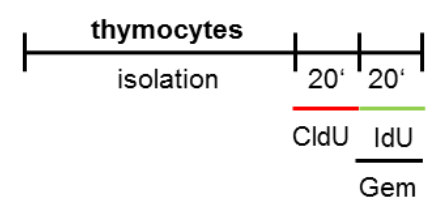

$\mathbf{F}$

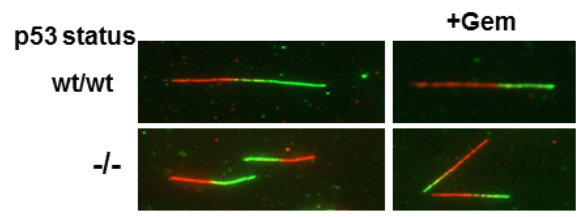

G

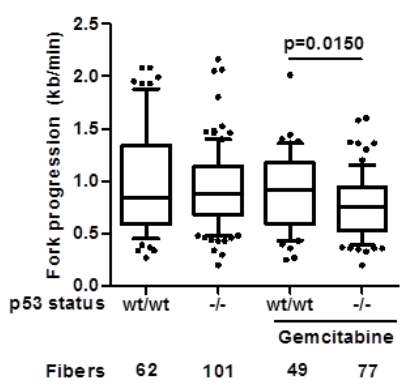

I

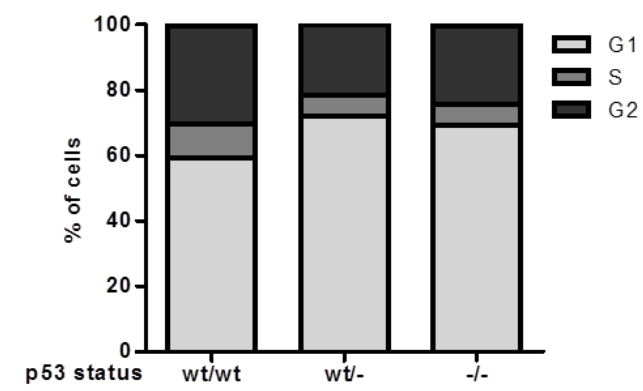

B

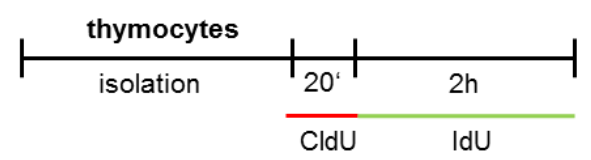

C

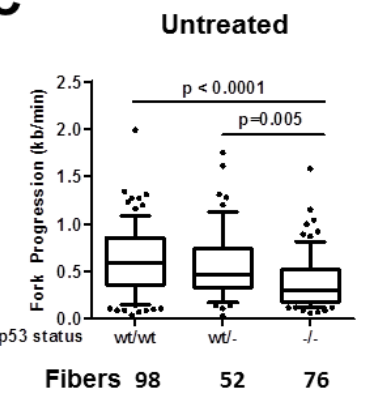

D Gemcitabine

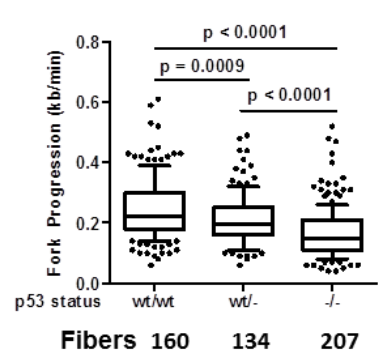

H
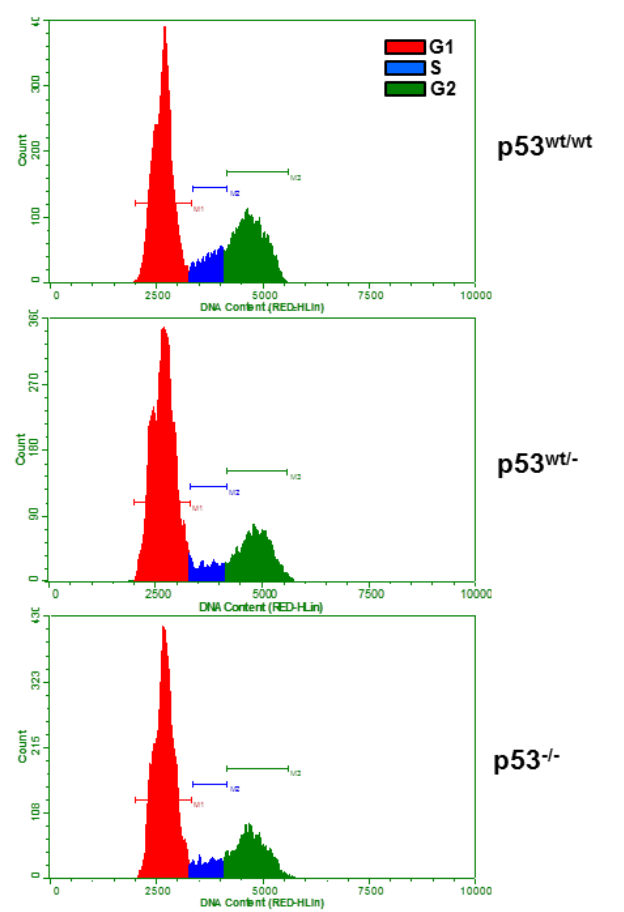

Suppl. Fig. V.I.7.7 Impact of p53 on DNA replication in thymocytes. Related to Fig. V.I.5

(A) p53 does not detectably affect mitochondrial ROS levels in thymocytes. Flow cytometry to detect ROS was carried out as in Suppl. Fig.V.I.7.4I. (B) Replicates from Fig.V.I.5B-E, in independent experiments using thymocytes from different mice. Thymocytes were isolated from mice with differernt p53 status (wild type, null, or heterozygous). Immediately after isolation, replicating DNA was labeled using $25 \mu \mathrm{M}$ CldU for 20 min, followed by $25 \mu \mathrm{M}$ IdU for $2 \mathrm{~h}$. Fiber lengths are displayed as blox plots in Panel C. The results from an analogous experiment, but with $400 \mathrm{nM}$ gemcitabine added to the second label, are shown in Panel D. (E)

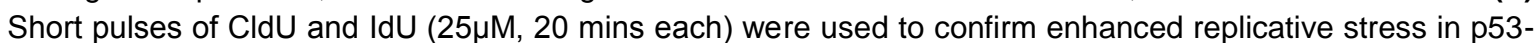
deficient thymocytes. Gemcitabine was added together with the IdU label as indicated. (F) Immunostaining was performed to detect the (short) labeled tracks of CldU (red) and IdU (green). 
Suppl. Fig. V.I.7.7 continued (G) Despite the short 2nd pulse and concomitant decreased likelihood of fork stalling, reduced fork progression was observed when evaluating the length of the IdU-labeled tracks in gemcitabine-treated cells. (H) The cell cycle distribution of the thymocytes was determined by propidium iodide and flow cytometry, and summarized in Panel I. No increase in S phase fractions was observed in p53deficient thymocytes. 


\section{V.II Manuscript}

\section{The chromatin modifiers Mdm2 and RNF2 prevent RNA:DNA- hybrids that impair DNA replication}

Ina Klusmann ${ }^{1}$, Federico Teloni ${ }^{2}$, Matthias Altmeyer ${ }^{2}$ and Matthias Dobbelstein ${ }^{1^{*}}$

3) Institute of Molecular Oncology, Göttingen Center of Molecular Biosciences (GZMB), University Medical Center Göttingen, D-37077 Göttingen, Germany

4) Department of Molecular Mechanisms of Disease, University of Zurich, $\mathrm{CH}-8507$ Zurich, Switzerland

${ }^{*}$ Corresponding Author. Correspondence and requests for materials should be addressed to M. D. (e-mail: mdobbel@uni-goettingen.de)

Highlights:

- Mdm2 as well as Polycomb Repressor Complexes support DNA replication.

- Mdm2 prevents replication stress through its Ring finger but independent of p53

- Upon Mdm2 depletion, RNA:DNA hybrids (R-loops) accumulate.

- R-loops are responsible for replication stress when Mdm2 or RNF2 are removed.

This manuscript has been peer-reviewed and is currently in revision for publication at PNAS (July 2018).

\section{Contributions to manuscript}

Contributions to the manuscript including Figures and corresponding Suppl. Figures 1-4 and 6-7 as well as illustrations, layout and text. 


\section{V.II.1 Abstract}

The p53-Mdm2 system is key to tumor suppression. We have recently reported that p53 as well as Mdm2 are capable of supporting DNA replication fork progression. On the other hand, we found that Mdm2 is a modifier of chromatin, modulating polycomb repressor complex (PRC)-driven histone modifications. Here we show that, similar to Mdm2knockdown, the depletion of PRC members impairs DNA synthesis, as determined in fiber assays. In particular, the ubiquitin ligase and PRC1 component RNF2/Ring1B is required to support DNA replication, similar to Mdm2. Moreover, the Ring finger domain of Mdm2 is not only essential for its ubiquitin ligase activity, but also for proper DNA replication. Strikingly, Mdm2 overexpression can rescue RNF2 depletion with regard to DNA replication fork progression, and vice versa, strongly suggesting that the two ubiquitin ligases perform overlapping functions in this context. The depletion of Mdm2 elicits the accumulation of RNA/DNA hybrids, suggesting R-loop formation as a mechanism of impaired DNA replication. Accordingly, RNase $\mathrm{H}$ overexpression or the inhibition of the transcription elongation kinase CDK9 each rescues DNA replication upon depletion of Mdm2 or RNF2. Taken together, our results suggest that chromatin modification by Mdm2 and PRC1 ensures smooth DNA replication through the avoidance of R loop formation. 


\section{V.II.2 Significance Statement}

Accurate DNA replication is a pre-requisite for cell proliferation and genetic stability, but obstacles to smooth replication fork progression are frequent. The oncogenic activity of Mdm2 has been largely ascribed to its ability of antagonizing the tumor suppressor p53. This report, however, points out a p53-independent activity of Mdm2 in suppressing Rloops, a structure that includes DNA:RNA hybrids and has recently emerged as a key obstacle to DNA replication. Accordingly, Mdm2 is required for sustaining DNA replication. Our results also reveal that Mdm2 and the Polycomb Repressor Complexes act in parallel to not only modify histones but also support DNA replication. Thus, chromatin modifiers that were traditionally implied in transcription regulation are enabling unperturbed DNA replication as well. 


\section{V.II.3 Introduction}

The tumor suppressor p53 is unique with regard to its mutation rate in human malignancies, exceeding $50 \%$. Its activity is balanced by the ubiquitin ligase Mdm2, the product of a p53-inducible gene. Some tumors overexpress or activate Mdm2 as part of an oncogenic mechanism, most notably to antagonise p53.

More recently, however, p53-independent activities of Mdm2 were discovered. Our lab described a chromatin modifier function of Mdm2 which contributes to histone $\mathrm{H} 2 \mathrm{~A}$ ubiquitination at $\mathrm{K} 119$, as well as to histone $\mathrm{H} 3$ trimethylation at $\mathrm{K} 27$ (Wienken et al., 2016, 2017). Accordingly, Mdm2 physically associates with the protein complexes that confer these chromatin modifications, i. e. the members of the polycomb repressor complexes (PRCs) 1 (Wen et al., 2014) and 2 (Wienken et al., 2016). These modifications were mostly characterized as mediating transcriptional repression, along with stemness (Blackledge et al., 2015; Schwartz and Pirrotta, 2013).

On the other hand, we have also identified a p53 function that broadens the traditional view on the "guardian of the genome". On top of eliminating cells with damaged DNA by apoptosis or senescence, p53 is also capable of enhancing the processivity of DNA replication forks (Klusmann et al., 2016). Other groups reported similar findings, considering various mechanisms of how p53 might enhance DNA replication, e. g. through avoiding topological stress (Yeo et al., 2016), inducing DNA polymerase eta and translesion synthesis (Lerner et al., 2017), orchestrating fork restart (Roy et al., 2018) or enhancing the levels of pCDKN1A/p21 and its association with PCNA (Mansilla et al., 2016). In other systems, p53 can also compromise DNA replication through p53associated exonuclease activity and DNA polymerase iota (Hampp et al., 2016), and forced CDKN1A/p21 synthesis impairs DNA replication in UV-irradiated cells (Mansilla et al., 2013). These findings are at least not easy to reconcile, underscoring the need for further investigations on how the p53-Mdm2-system affects DNA replication. In our hands, the p53 target gene product Mdm2 is supporting DNA replication fork progression, similar to p53 itself (Klusmann et al., 2016). This suggests that the induction of Mdm2 constitutes a major mechanism of how p53 supports replication. This notion, however, did not answer the question by what mechanism(s) Mdm2's impact on DNA replication could be explained. 
Conflicts between transcription and DNA replication appear to represent a major cause of replication stress (Bermejo et al., 2012). Such conflicts may not simply result from collisions between RNA- and DNA-polymerases, but rather from the co-transcriptional occurrence of DNA:RNA hybrids with an additional single DNA strand, so-called R-loops. R-loops seem to hinder DNA replication forks from progressing (reviewed in Aguilera and Garcia-Muse 2012). This raises the possibility that chromatin modifications, e. g. through Mdm2 or PRCs (Wienken et al., 2016), might help to avoid R-loops and ease DNA replication fork progression.

Here we show that Mdm2 and PRCs act similarly to sustain DNA replication fork progression. The Ring finger domain of Mdm2, conferring the ubiquitin ligase function, is essential for smooth DNA replication. Furthermore, Mdm2 and the PRC1 component RNF2 can substitute for each other in this function, suggesting that both ubiquitin ligases employ similar mechanisms to facilitate DNA replication through chromatin modification. And indeed, the depletion of either ubiquitin ligase induces R-loops, whereas DNA replication is restored by RNase-mediated removal of R-loops. Integrating these findings, we propose that Mdm2 and PRC1 contribute to DNA replication fork processivity through the avoidance of R-loops by chromatin modification. 


\section{V.II.4 Results}

\section{V.II.4.1 Depletion of Mdm2 or Polycomb repressor complex 2 members similarly decreases DNA replication fork progression}

Our previously published results suggested that Mdm2 induces chromatin modifications with a pattern analogous to PRCs, through a mechanism that involves the physical association between Mdm2 and PRC2 (Wienken et al., 2016). On the other hand, we observed that p53 and Mdm2 are required to support DNA replication fork progression (Klusmann et al., 2016). To understand whether the chromatin modifier activity of Mdm2 might also be involved in the support of DNA replication, we first compared the depletion of Mdm2 and PRC2 members regarding their impact on DNA replication fork progression (Fig.V.II.1A-B).
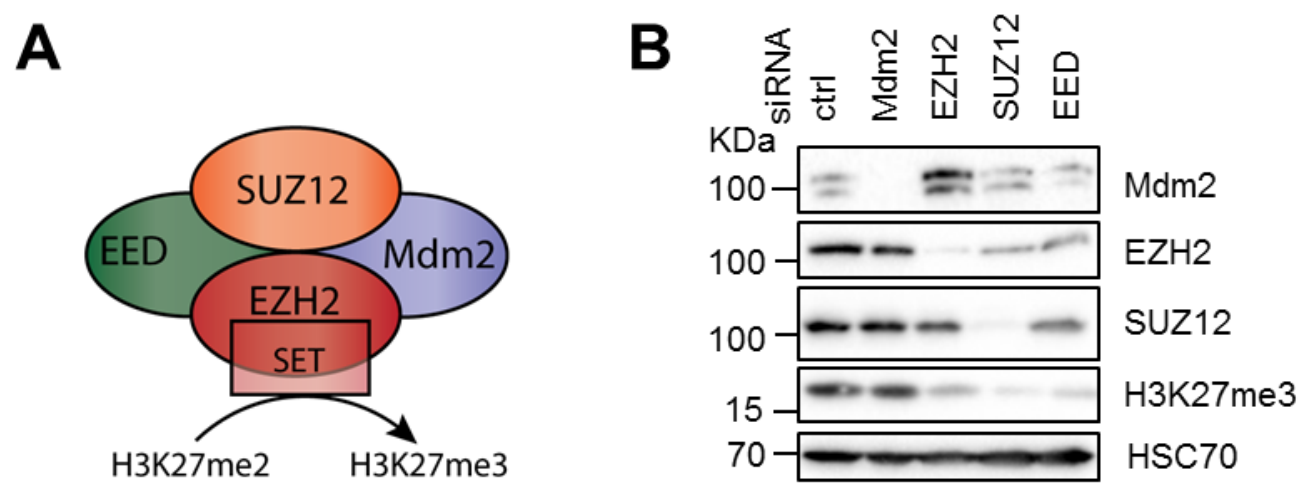

Fig.V.Il.1 Compromised DNA replication fork progression upon depletion of Mdm2 or PRC2 members

(A) Schematic diagram of the main components of the PRC2 complex and its interaction partner Mdm2 that have been subjected to replication studies. siRNA transfections targeting Mdm2, EZH2, SUZ12, and EED as well as an inhibitor targeting the SET domain of EZH2 were used. (B) H1299 cells were transfected with two siRNAs each against the targets Mdm2, EZH2, SUZ12, and EED for 48 hours and subjected to immunoblot analysis. Knockdowns were confirmed by staining for the target proteins as well as the target of active PRC2, i. e. trimethylated histone 3 (H3K27me3).

To address this, we employed the p53-/- lung large cell carcinoma cell line H1299 (Gazdar et al., 2010) to knock down Mdm2 or the PRC2 components EZH2, EED, or SUZ12 by siRNA, followed by fiber assays for DNA replication (Fig.V.II.1C, Suppl. Fig.V.II.1A-G). Strikingly, the depletion of all tested PRC2 components led to a marked decrease in DNA replication fork progression, to a similar extent as the removal of Mdm2 (Fig.V.II.1E-D). 


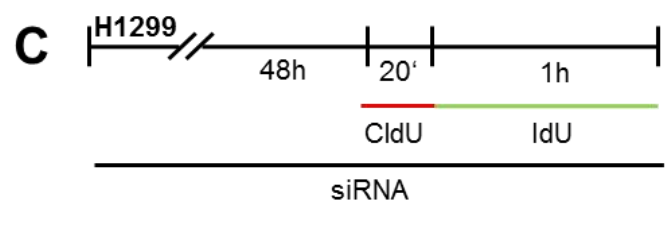

$\mathbf{E}$

D $\quad$ siRNA
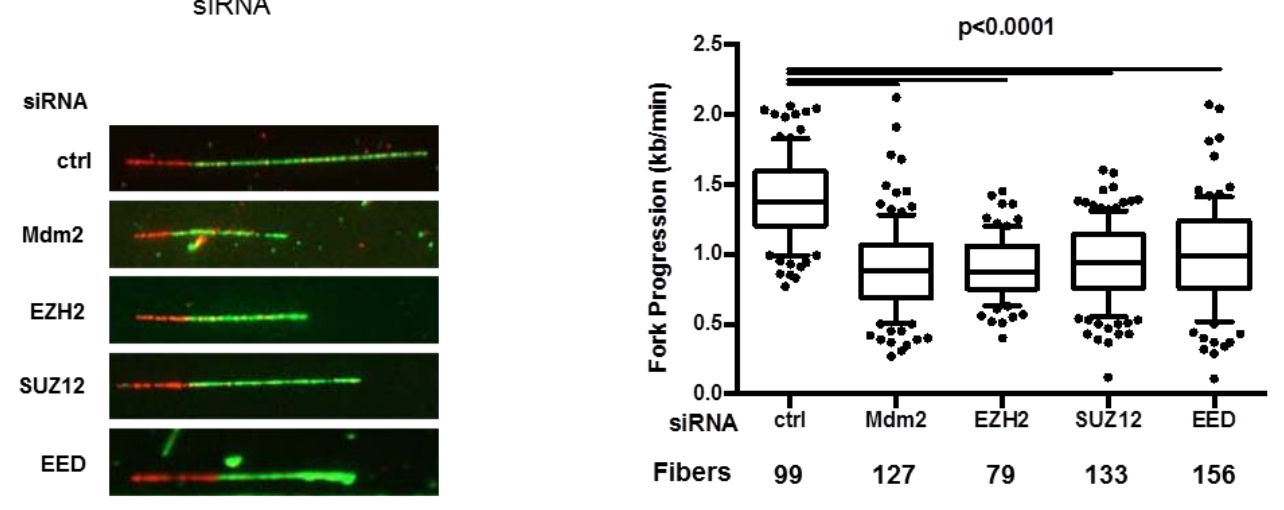

Fig.V.Il.1 Compromised DNA replication fork progression upon depletion of Mdm2 or PRC2 members.

(C) Cells were treated as in (B) and incubated with 5'-chloro-2'-deoxy-uridine ( $25 \mu \mathrm{M} \mathrm{CldU,} 20 \mathrm{~min})$ followed by iodo-deoxy-uridine ( $25 \mu \mathrm{M} \mathrm{IdU,} 60 \mathrm{~min})$ as indicated. (D) Tracks of newly synthesized DNA were visualized by immunostaining of CldU (red) and IdU (green). (E) Fork progression was determined from the track length of the second label (IdU; $\mathrm{kb} / \mathrm{min}$ ) and displayed in a boxplot with 10-90 percentile whiskers.

In the case of EZH2, this is consistent with a recently published result indicating that EZH2-depleted cells resume replication to a lesser extent upon temporary fork stalling (Rondinelli et al., 2017). Similarly, a pharmacological inhibitor of EZH2 catalytic activity as a histone methyl transferase compromised DNA replication (Fig.V.II.1F-I, Suppl. Fig.V.II.1I-M). Thus, interfering with PRC2 and its activity phenocopies the impact of Mdm2 depletion on DNA replication, providing a first hint that Mdm2 and PRC2 might affect DNA replication by similar pathways.
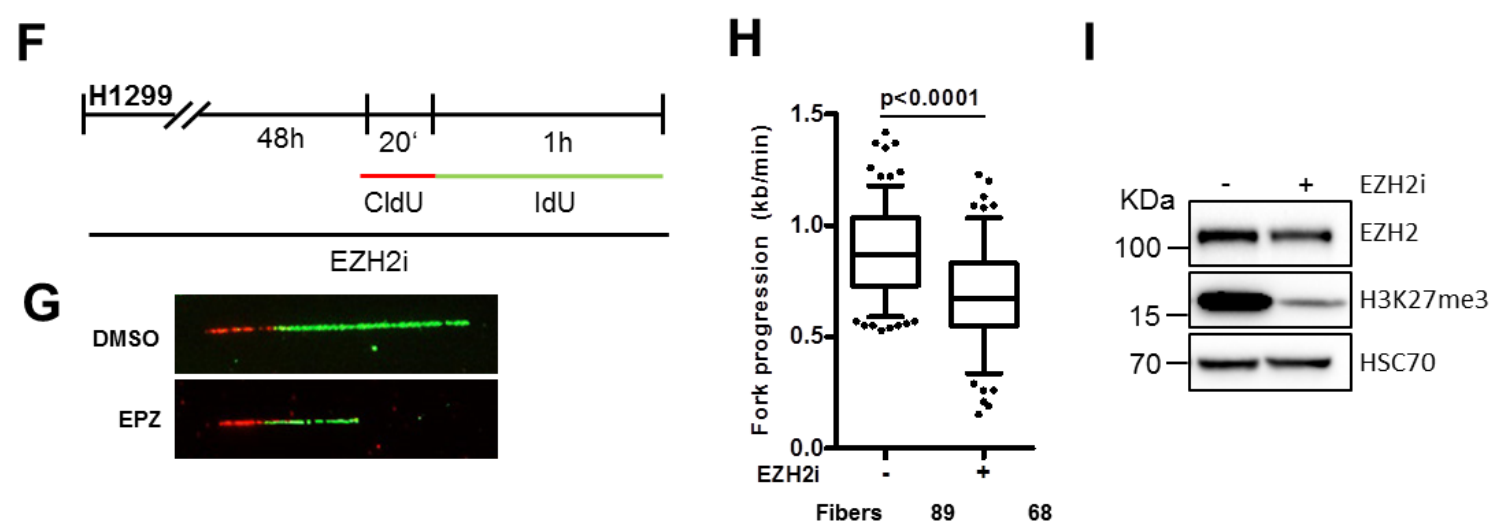

Fig.V.Il.1 Compromised DNA replication fork progression upon depletion of Mdm2 or PRC2 members.

(F) H1299 cells were treated with the EZH2 inhibitor EPZ-6438 at $5 \mu \mathrm{M}$ for 48 hours, followed by incubation with CldU and IdU as in (C). (G) Representative labelled tracks were immunostained as described in (D). (H) Boxplot analysis of the fork progression during the time of IdU label with 10-90 percentile whiskers.(I) Cells were treated as in $(\mathrm{F})$ and subjected to immunoblot analysis of $\mathrm{H} 3 \mathrm{~K} 27 \mathrm{me} 3$ as a readout for inhibitor activity. 


\section{V.II.4.2 Interfering with the polycomb repressor complex 1 slows down DNA replication forks and cell proliferation}

Besides the trimethylation of histone $\mathrm{H} 3$ at $\mathrm{K} 27$, Mdm2 also supports the ubiquitination of histone H2A at K119 (Wienken et al., 2016), perhaps as a result of direct ubiquitin ligase activity (Minsky and Oren, 2004). This site is mostly known for its ubiquitination by PRC1, typically through its RNF2 component (Blackledge et al., 2015). To test whether RNF2 knockdown might affect DNA replication in a similar manner as Mdm2 depletion, we performed fiber assays and found reduced DNA replication fork progression again (Fig.V.II.2A-E, Suppl. Fig.V.II.2A-B). Analogous observations were made in the presence of a pharmacological inhibitor of Bmi1, another PRC1 component (Fig.V.II.2F-H, Suppl Fig. V.II.2C-D).

A

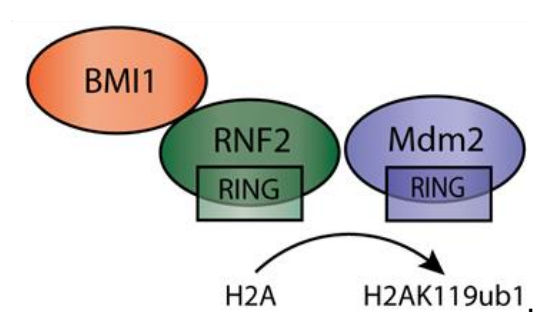

B

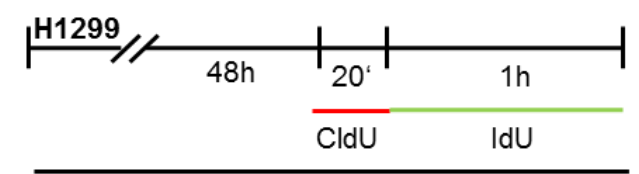

siRNA/inhibitor

C

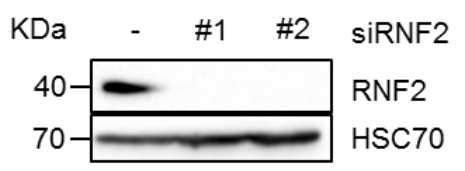

D

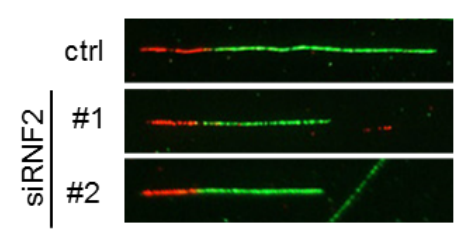

E

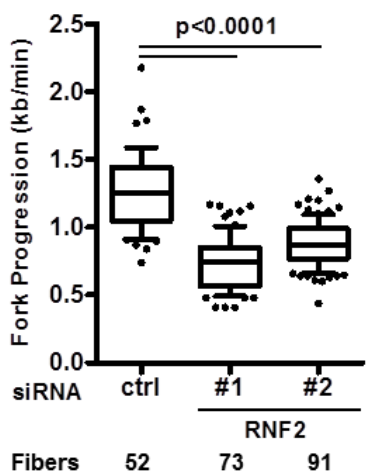

Fig.V.Il.2 Decreased fork progression upon RNF2 depletion or Bmi1 inhibition.

(A) Diagram of the catalytic subunit of the PRC1 complex, RNF2, that was targeted by siRNA transfections as well as another component of the complex, BMI1, targeted by an inhibitor. (B) H1299 cells were depleted of endogenous RNF2 by siRNA transfection for 48 hours and labelled with CldU (20 $\mathrm{min}$ ) and IdU (60 $\mathrm{min}$ ) for fiber analysis. (C) Immunoblot analysis of RNF2 after treatment described in (B), confirming the depletion of RNF2 with two siRNAs. (D) Representative images of labelled tracks after immunostaining of CldU (red) and IdU (green). (E) Boxplot analysis of IdU-labelled tracks upon RNF2 depletion with 10-90 percentile whiskers. 

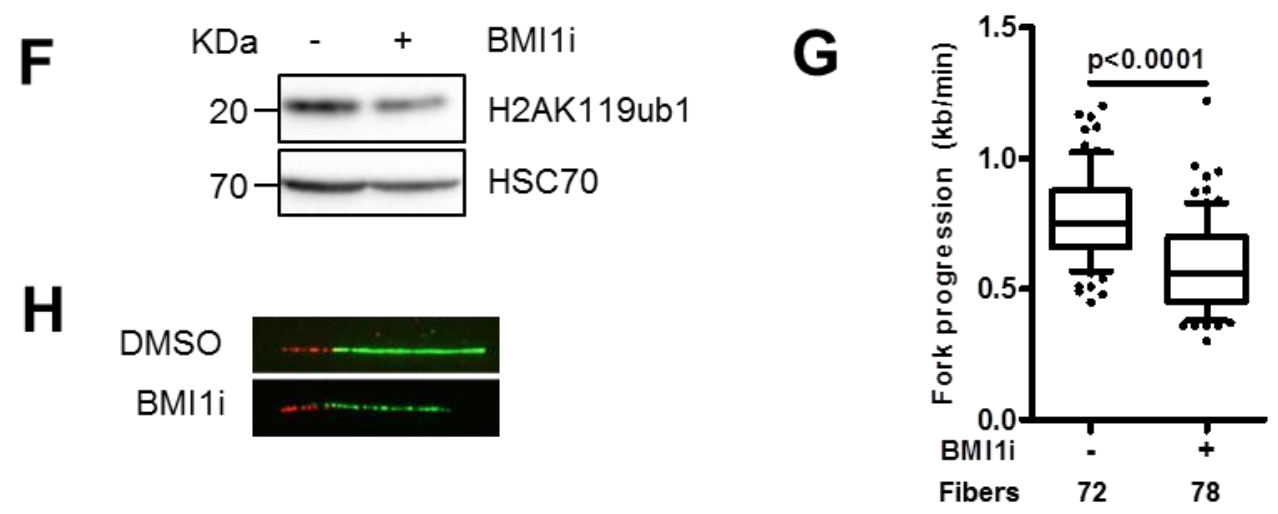

Fig.V.II.2 Decreased fork progression upon RNF2 depletion or Bmi1 inhibition.

(F) $\mathrm{H} 1299$ cells were treated with $1 \mu \mathrm{M}$ of the BMl1 inhibitor PTC-209 for 48 hours, followed by immunoblot analysis detecting monoubiquitinated histone 2A (H2AK119ub1) as a readout of inhibitor activity. (G) Fork progression analysis of the IdU label in cells treated with $1 \mu \mathrm{M}$ BMl1i for 48 hours, with representative images in $\mathbf{( H )}$.

In order to assess whether the differences seen in fork progression rates are a result of increased fork stalling or decreased velocity of the replication fork, we applied a modified labelling protocol for DNA fiber assays as described in (Klusmann et al., 2016), changing the label of newly synthesized DNA for multiple brief periods of time, allowing the exact determination when a replication fork had stalled. Mdm2 depletion led to an increase in fork stalling indicated by fibers with less than 7 labels incorporated (Fig.V.II.2I-L). Fork velocity in continuously replicating stretches (labels 2-5 only) was affected by either depleting Mdm2 or RNF2 from the cells (Fig.V.II.2M). In conclusion, Mdm2 is required for proper DNA replication fork processivity and also velocity, whereas RNF2 primarily contributes to fork velocity. 
I

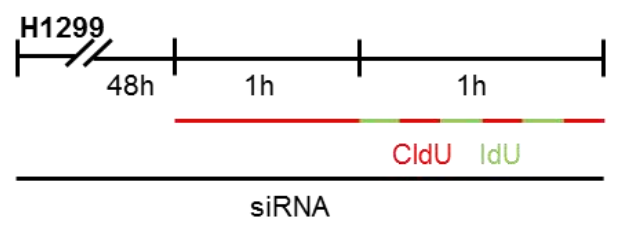

$\mathbf{J}$

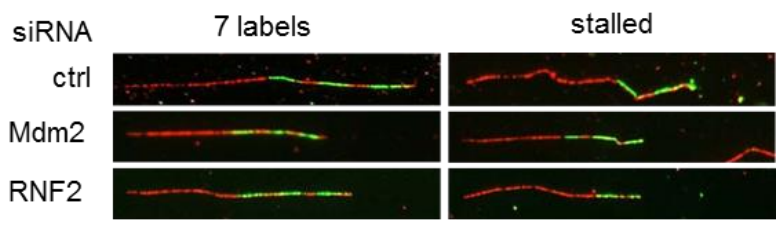

K

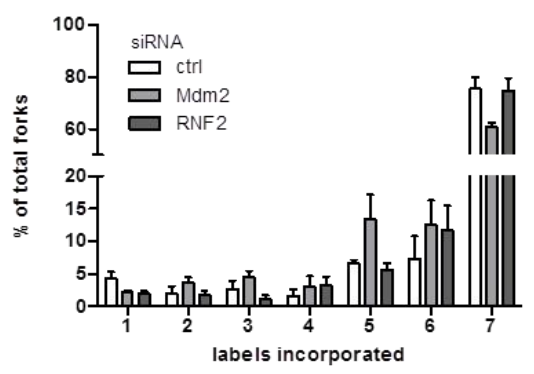

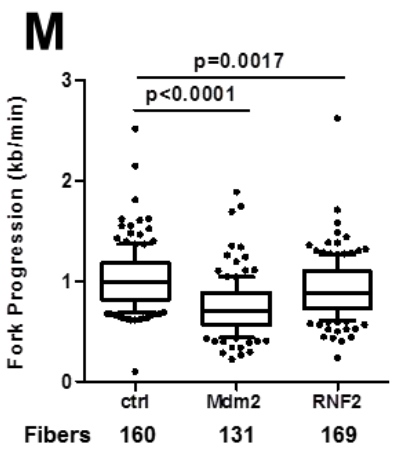

Fig.V.II.2 Decreased fork progression upon RNF2 depletion or Bmi1 inhibition.

(I) Fork processivity was analyzed as described previously (Klusmann et al. 2016). First, cells were transfected with scrambled siRNA (control) or siRNAs targeting Mdm2 and RNF2 for 48 hours and then labelled with CldU for 1 hour, followed by short (10 minute) pulses of IdU and CldU for a total of 7 labels. From this, the length of labels 2-5 is used for fork progression analysis and the number of labels incorporated for fork stalling analysis. (J) Representative images of replicated stretches that have incorporated all 7 labels as well as stalled ones that contain less than 7 labels. (K) The number of forks that proceeded through $n$ labels is displayed for control, Mdm2 and RNF2 knockdown. 7 labels reflect full progression of the fork throughout the entire labelling time. Numbers lower than 7 indicate premature termination during the labelling time. (L) The percentages of forks with less than 7 labels indicate that Mdm2 and RNF2 knockdowns cause replication to run in a less processive manner than cells transfected with scrambled (control) siRNA. (M) Fork velocity was determined through the length of labels two to five (kilobases/min) which ensures that fork stalling (as seen in $(\mathrm{K})$ and $(\mathrm{L}))$ is not affecting velocity measurements.

In addition to direct effects on replication forks, cells depleted of Mdm2 and RNF2 were also less able to give rise to progeny (Fig.V.II.2N, Suppl. Fig.V.II.2E-G). This effect was also seen when exogenous replicative stress was induced by a 24 hour gemcitabine treatment (Fig.V.II.2O). 
$\mathbf{N}$

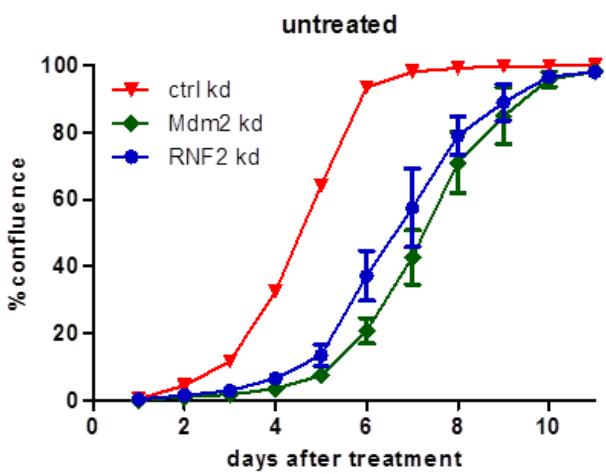

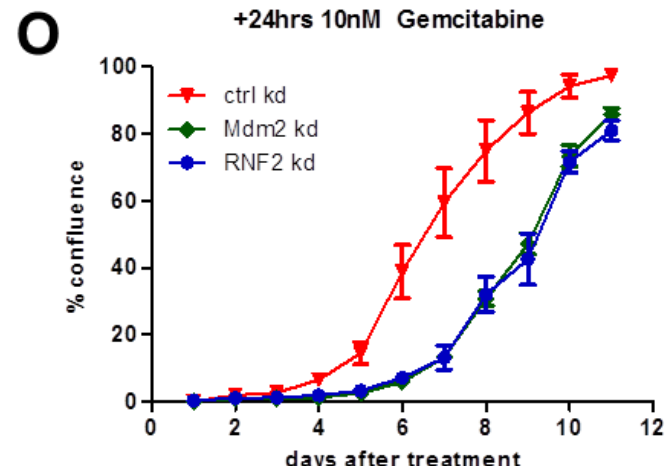

Fig.V.II.2 Decreased fork progression upon RNF2 depletion or Bmi1 inhibition.

H1299 cells were transfected with siRNAs against Mdm2 and RNF2. 24 hours post transfection, cells were treated with 10 nM Gemcitabine (in $\mathrm{H}_{2} \mathrm{O}$ ) for another 24 hours. Confluence of transfected cells (N) and with additional Gemcitabine treatment $(\mathbf{O})$ was analysed every 24 hours.

\section{V.II.4.3 The Ring finger domain of Mdm2 is necessary to sustain DNA replication}

The chromatin modifier function of Mdm2 requires its Ring finger domain, suggesting that the E3 ubiquitin ligase activity of this domain is involved (Wienken et al., 2016). We therefore sought to test whether the same is true for the support of DNA replication by Mdm2. We first employed murine embryonic fibroblasts (MEFs) from animals that either had wild type mdm2 on a p53-/- background, or otherwise lacked both mdm2 and p53, or had the p53-/- background with a biallelic point mutation in the Mdm2 gene that gave rise to the mutation C464A, disrupting the Ring finger structure (Itahana et al., 2007) (Fig.V.II.3A). Strikingly, the knock-out of Mdm2, as well as the Ring finger knock-in, each led to reduced DNA replication fork progression compared to p53 single knockout cells (Fig.V.II.3B-C, Suppl. Fig.V.II.3A-B). Similar results were obtained with $\mathrm{H} 1299$ cells. While Mdm2 overexpression had little effect on DNA replication, the overexpression of Mdm2 with a Ring finger mutation markedly reduced fork progression, presumably as a result of a dominant negative effect (Fig.V.II.3D-G, Suppl. Fig.V.II.3C-D). Taken together, these results strongly suggest that Mdm2 requires the Ring finger domain and its ubiquitin ligase activity to sustain DNA replication independent of p53. 
A

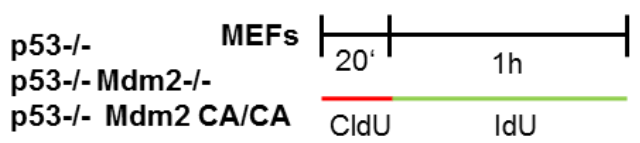

C

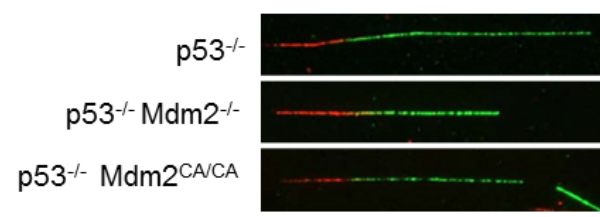

D

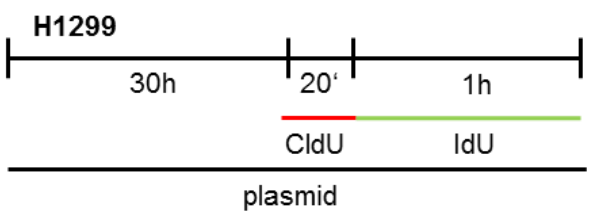

$\mathbf{F}$

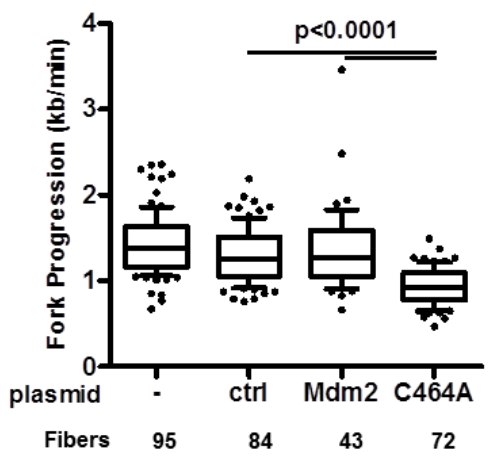

B

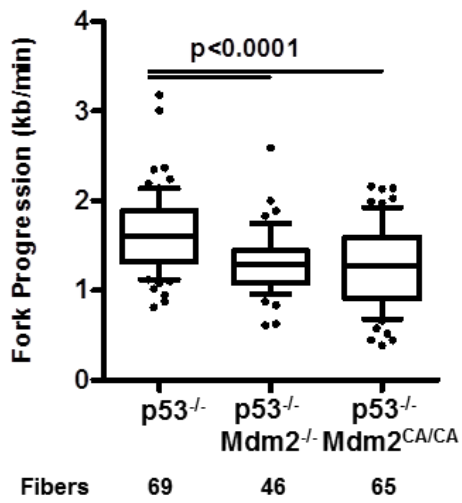

$\mathbf{E}$

plasmid

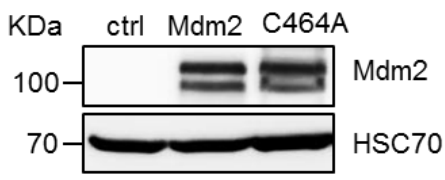

G

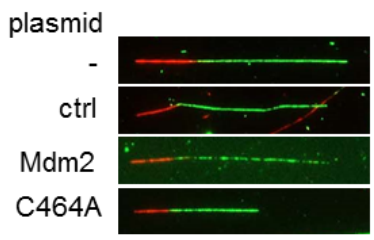

Fig.V.II.3 Requirement for the Ring finger domain of Mdm2 to support replication fork progression.

(A) Mouse embryonic fibroblasts lacking p53, alone or in combination with a deletion of Mdm2 or a biallelic point mutation in the RING domain of Mdm2 (C462A), were subjected to fiber assay labelling with CldU (20 $\mathrm{min}$ ) and IdU (60 min). (B) Boxplot analysis o fork progression in the IdU label when either Mdm2 or just its RING domain were deleted with 10-90 percentile whiskers. (C) Representative images of the labelled tracks of CldU (red) and IdU (green). (D) H1299 cells were subjected to plasmid transfection for 30 hours, followed by DNA fiber assay labelling with CldU for 20 minutes and IdU for 60 minutes. (E) Immunoblot analysis upon transfection as in (D) confirms overexpression of Mdm2 with both a wildtype and a mutant plasmid carrying a point mutation in the RING finger. (F) Analysis of fork progression in the IdU label shows a significant reduction upon overexpression of RING-mutant Mdm2 but not wild type Mdm2. (G) Fluorescently labelled tracks of CldU (red) and IdU (green) in untransfected samples as well as upon transfection with of the control plasmid pcDNA3 or the expression plasmids pCMV-Mdm2 and pCMV-Mdm2C464A (note that human Mdm2 carries the corresponding cysteine residue that forms part of the Ring finger structure at position 464). 


\section{V.Il.4.4 Mdm2 is capable of rescuing DNA replication when RNF2 is depleted.}

To further explore whether Mdm2 might support DNA replication by a similar mechanism as PRC2, we performed rescue experiments upon knockdown of Mdm2 or RNF2 (Fig.V.II.4A). As expected, the overexpression of each component reconstituted DNA replication when it had been depleted by siRNA. (Fig.V.II.4B-G, Suppl. Fig.V.II.4A-C). Although the siRNA could also target the overexpressed Mdm2, the remaining amounts of Mdm2 still exceeded the endogenous levels, explaining the rescue of DNA replication (Fig.V.II.4D). Of note, however, the overexpression of Mdm2 also rescued DNA replication when RNF2 had been depleted (Fig.V.II.4H-J, Suppl. Fig.V.II.4D) while overexpression of RNF2 partially restored fork progression upon Mdm2 knockdown (Fig.V.II.4K-M; Suppl. Fig. V.II.4E).

Thus, enhanced Mdm2 levels can compensate for a lack of RNF2 and vice versa. Since both proteins are capable of enhancing H2A ubiquitination at K119 (Minsky and Oren, 2004; Wienken et al., 2016), this at least suggests that the ubiquitination of this histone enables smooth DNA replication. 
A
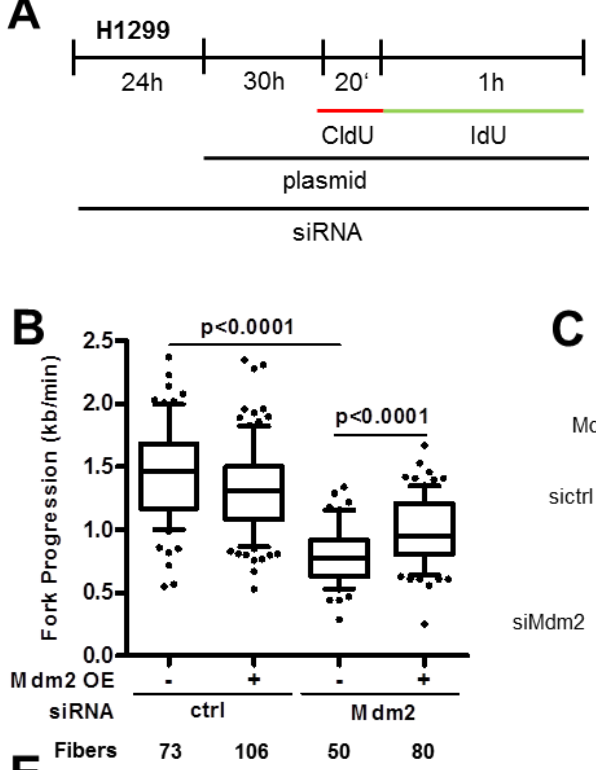

C D
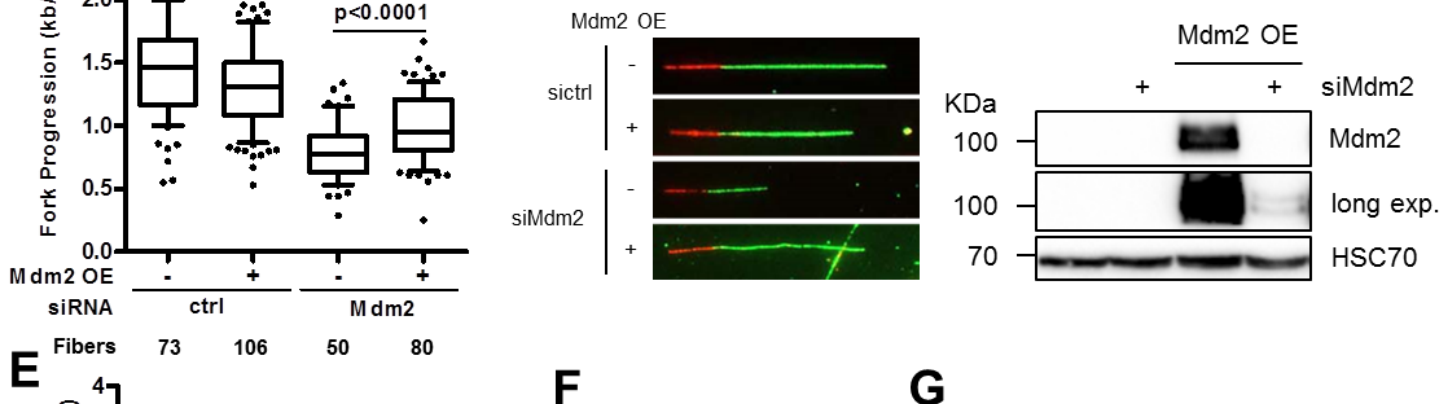

$\mathbf{F}$

G
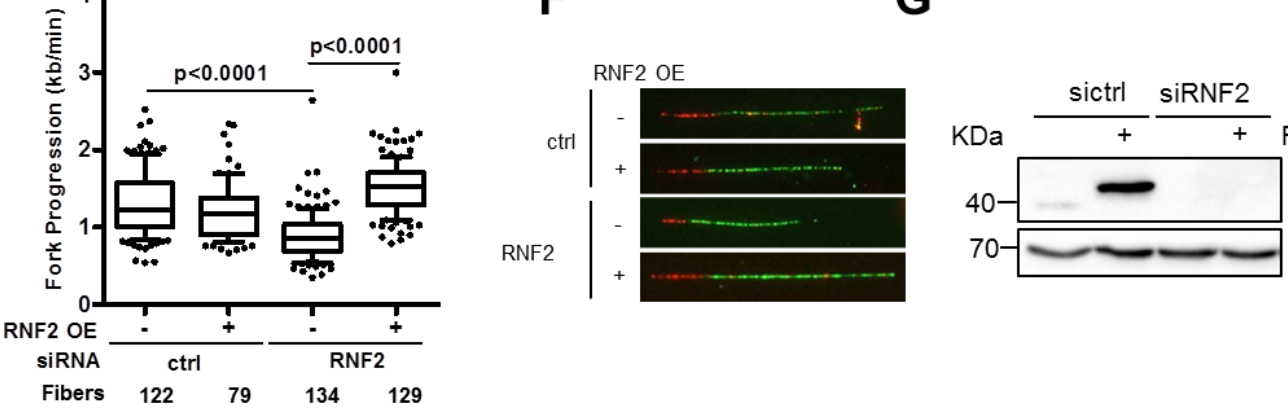

RNF2 OE

RNF2

HSC70

Fig.V.II.4 Mutual rescue of fork progression in Mdm2- and RNF2-depleted cells.

(A) $\mathrm{H} 1299$ cells were first transfected with siRNAs, followed by a plasmid transfection after 24 hours. After another 30 hours, samples were subjected to fiber assay labelling with $25 \mu \mathrm{M}$ CldU $(20 \mathrm{~min})$ and $25 \mu \mathrm{M} \mathrm{IdU}(60$ $\mathrm{min})$. (B) Boxplot analysis of fork progression after Mdm2 depletion and plasmid transfection with pcDNA3 and pCMV-Mdm2 with representative images of labelled tracks in (C). (D) Immunoblot analysis of samples after transfection as in (A) confirms the plasmid transfection as well as Mdm2 depletion by siRNA. A longer exposure reveals the presence of Mdm2 that still exceeds the endogenous levels when combining the siRNA with overexpression. (E) Fork progression analysis after RNF2 depletion and transfection with RNF2 plasmid, with representative images in (F). (G) Immunoblot analysis of RNF2 overexpression (as in (E)). 


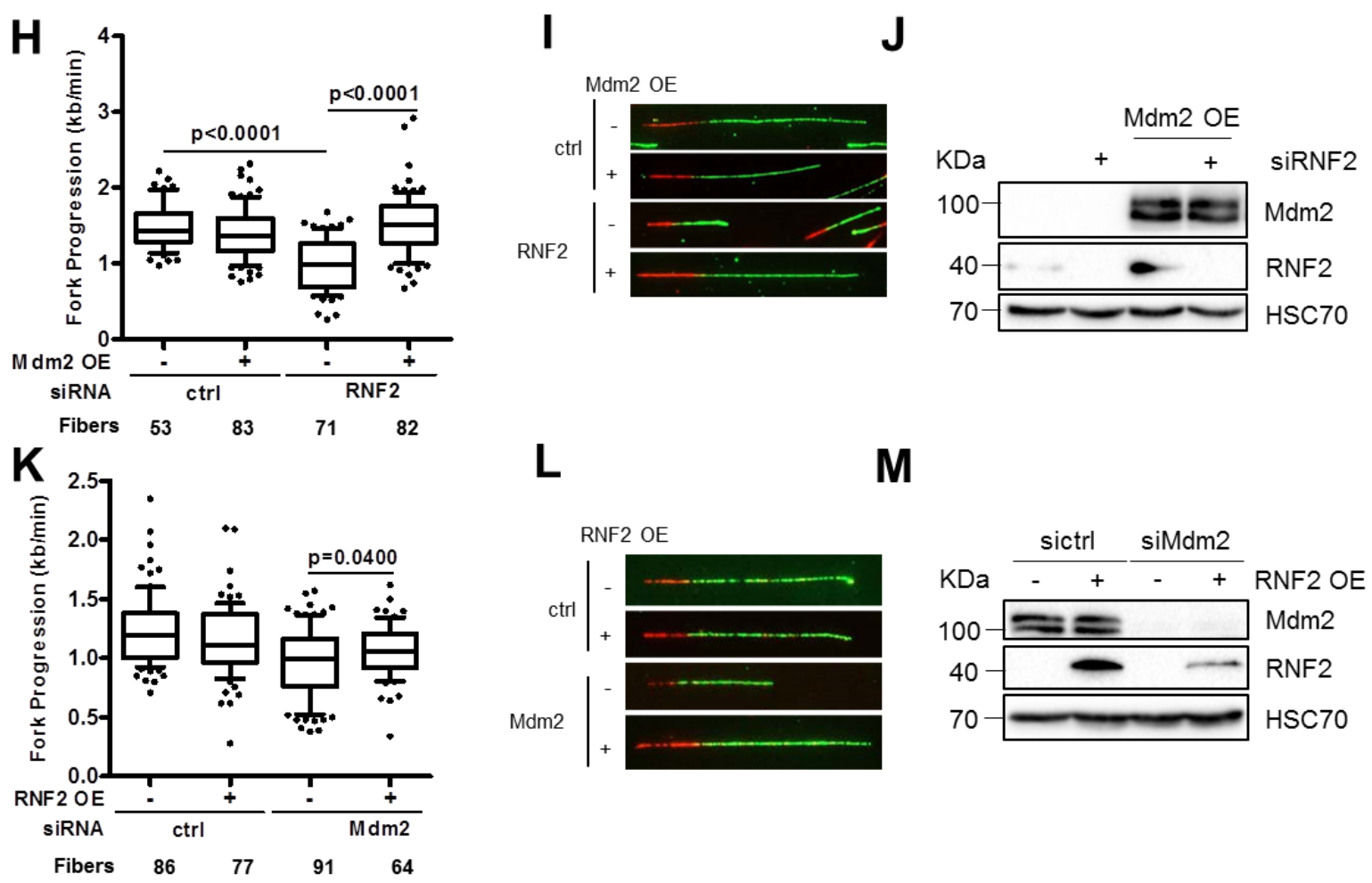

Fig.V.Il.4 Mutual rescue of fork progression in Mdm2- and RNF2-depleted cells.

(H) Fork progression in the IdU label after RNF2 depletion and plasmid transfection to overexpress Mdm2. (I) Fluorescently labelled tracks of CldU (red) and IdU (green) displayed as boxplots with 10-90 percentile whiskers. (J) Immunoblot staining for Mdm2 and RNF2 confirms knockdown and overexpression described in (H). (K) Analysis of fork progression during the IdU label after Mdm2 depletion and RNF2 overexpression. (L) Representative labelled tracks of CldU (red) and IdU (green). (M) Immunoblot analysis confirms Mdm2 depletion and overexpression of RNF2.

\section{V.II.4.5 Mdm2 depletion enhances R-loop formation}

In search of a mechanism that might enhance DNA replication through histone ubiquitination, we next tested the role of R-loops, i. e. DNA:RNA hybrids that typically form during dysregulated transcription and can represent obstacles to DNA polymerases during DNA replication (Santos-Pereira and Aguilera, 2015). PRC-mediated histone modifications often repress transcription (Blackledge et al., 2015). In parallel, we had previously observed similar repression patterns and histone modifications as a function of Mdm2 (Wienken et al., 2016). Therefore, we reasoned that R-loop formation might occur in response to the depletion of PRC components or Mdm2, providing a plausible explanation for impaired DNA replication fork progression. Ribonuclease $\mathrm{H} 1(\mathrm{RNaseH} 1)$ is an enzyme that recognises RNA:DNA hybrid structures and cleaves the RNA moiety. Mutagenic modifications of the catalytic triad in its C-terminus render $\mathrm{RNaseH} 1$ 
catalytically inactive while retaining its RNA:DNA hybrid binding ability (Wu et al., 2001). We made use of an inducible cell system carrying the D210N mutation in GFP-tagged $\mathrm{RNaseH} 1$ to specifically detect R-loops by quantitative image based cytometry (Fig.V.II.5A-B; Nguyen et al., 2017; Pellegrino et al., 2017).

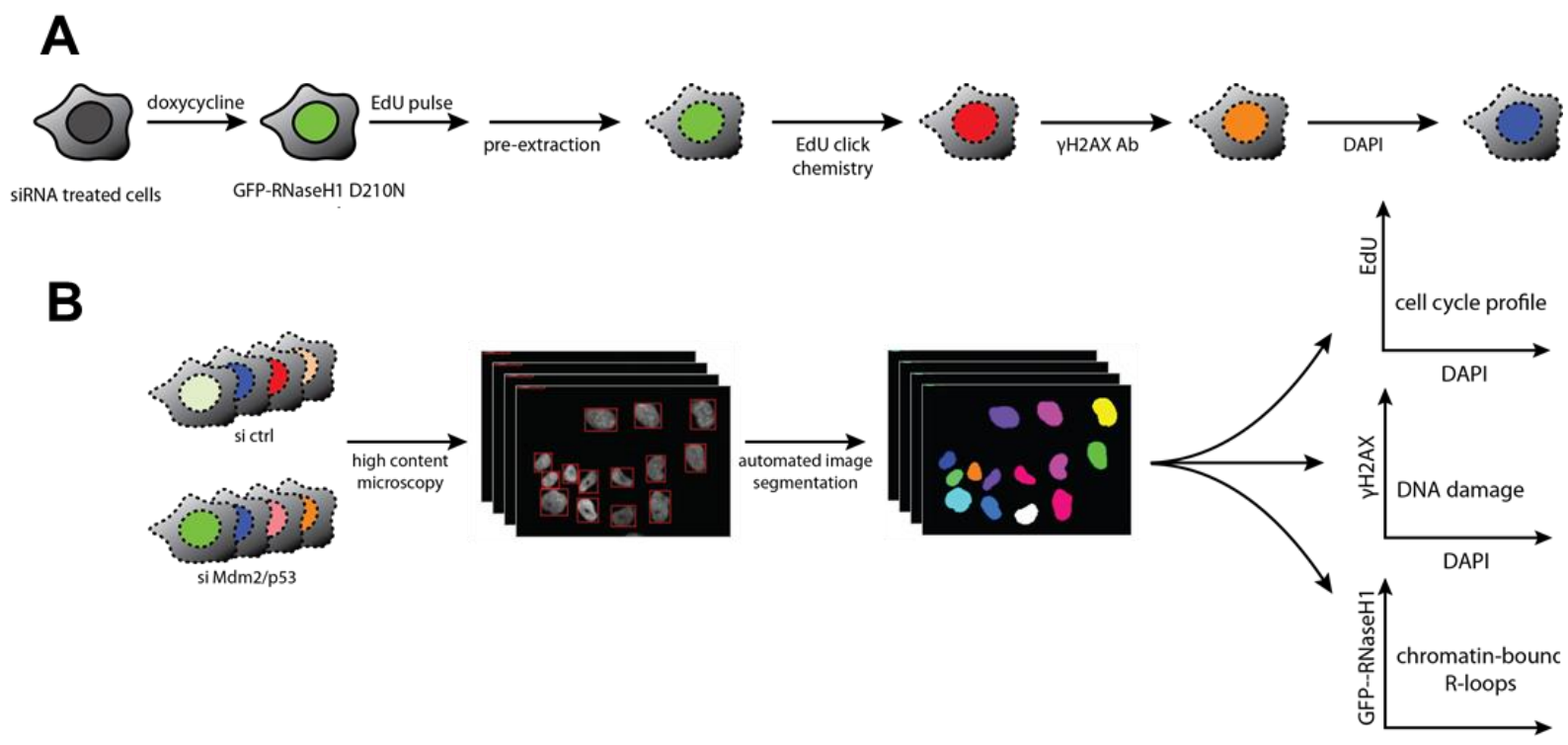

Fig.V.II.5 Mdm2 depletion increases replicative stress and R-loop formation.

(A) Schematic outline of the experimental setup for quantitative image based cytometry used to quantify DNA damage and R-loops upon depletion of Mdm2. U2OS cells that stably express the non-catalytic GFPRNaseH1-D210N mutant were transfected with siRNA against p53 and Mdm2 for 48 hours, prior to mutant $\mathrm{RNaseH} 1$ induction by doxycycline treatment for another 24 hours. Next, the cells were pulsed with EdU to detect DNA synthesis for 20 minutes, pre-extracted, fixed and stained. The signals from DAPI, EdU, $\mathrm{YH} 2 \mathrm{AX}$, and chromatin-bound RNaseH1-D210N were quantified by high-content microscopy. The D210N mutation abolishes RNase activity but retains binding to RNA:DNA hybrids and can thus be used to detect them. (B) Two-dimensional analysis of the fluorescent signals of EdU, $\mathrm{YH} 2 \mathrm{AX}$, and GFP-RNaseH1-D210N versus DAPI intensity allows cell cycle staging as well as S-phase-specific analysis of DNA damage and R-loops.

In parallel to R-loops, we also detected DNA synthesis and DNA damage signalling in the same system. In line with our fiber studies, EdU incorporation was reduced after depleting Mdm2 and p53 (Fig.V.II.5C-D, Suppl. Fig.V.II.5A-B), reflecting impaired DNA replication. Furthermore, we observed increased levels of the DNA damage marker $\mathrm{YH} 2 \mathrm{AX}$ (Fig.V.II.5E-F, Suppl. Fig.V.II.5C-D), again reflecting replication stress. Strikingly, the amounts of chromatin-bound RNase-H1-D210N were highly elevated in the absence of Mdm2, indicating that depletion of Mdm2 increases R-loop formation (Fig.V.II.5G-H, Suppl. Fig.V.II.5E-F). In conclusion, loss of Mdm2 increases the persistence of nonphysiological levels of R-loops. 
C

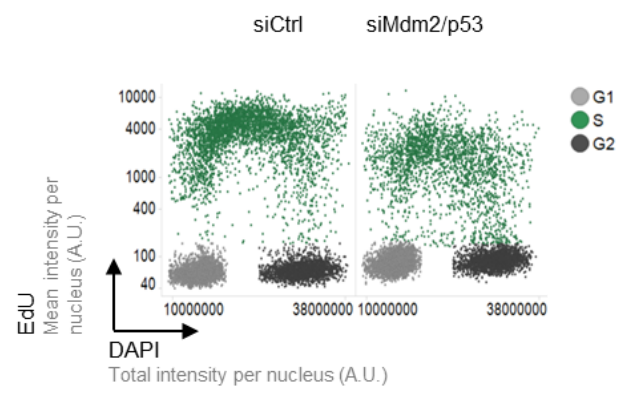

E

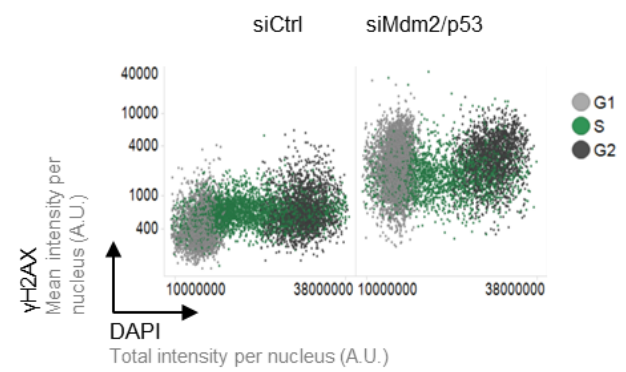

G

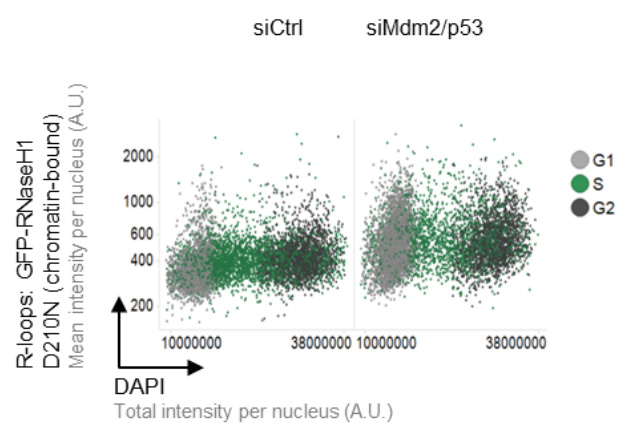

D

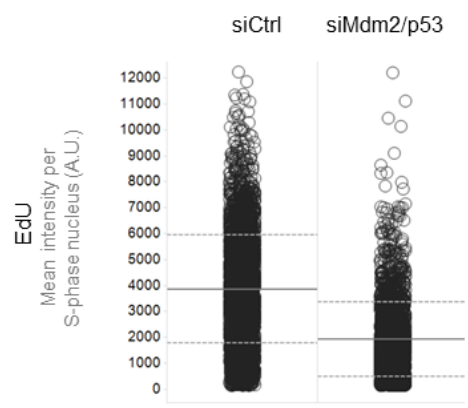

$\mathbf{F}$

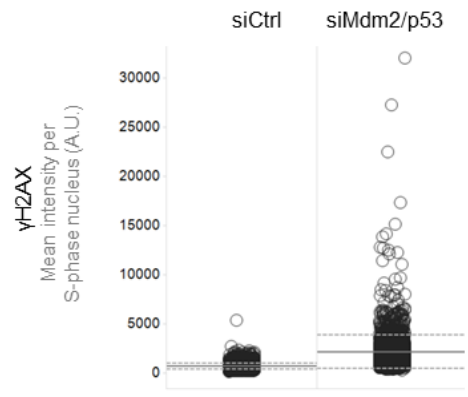

H

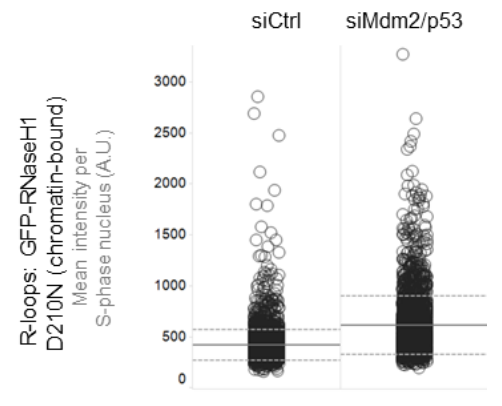

Fig. V.Il.5 Mdm2 depletion increases replicative stress and R-loop formation.

(B) Two-dimensional analysis of the fluorescent signals of EdU, yH2AX, and GFP-RNaseH1-D210N versus DAPI intensity allows cell cycle staging as well as S-phase-specific analysis of DNA damage and R-loops. For this analysis, the total number of cells analysed was $n=7545$ in control knockdown and 7412 in p53/Mdm2depleted cells (C, E, G). S-phase specific data was collected from $n=3457$ in controls and $n=2225$ in p53/Mdm2-depleted samples (D, F, H). (C) Two-dimensional cell cycle staging was performed based on the EdU and DAPI signals. (D) The S-phase specific EdU signals were plotted for Mdm2/p53-depleted cells versus a control siRNA transfection. Horizontal lines represent averages and standard deviations. (E) Levels of the DNA damage marker $\mathrm{yH} 2 \mathrm{AX}$ increase upon depletion of Mdm2 and p53 throughout the cell cycle. (F) The S-phase specific $\mathrm{YH} 2 \mathrm{AX}$ signals were plotted for Mdm2/p53-depleted cells versus a control siRNA transfection. Horizontal lines represent averages and standard deviations. (G) Chromatin-bound RNaseH1D210 marks RNA:DNA hybrids, and this signal increases when Mdm2 and p53 were depleted. (H) The Sphase specific signals of chromatin-bound RNaseH1-D210N were plotted for Mdm2/p53-depleted cells versus a control siRNA transfection. Horizontal lines represent averages and standard deviations. 


\section{V.II.4.6 Interfering with transcriptional elongation by CDK9 inhibition allows processive DNA replication despite the removal of Mdm2 or RNF2}

We next sought to determine whether conflicts with transcription cause replication stress when Mdm2 is depleted. One way of addressing these conflicts is by inhibiting CDK9, a kinase essential for transcriptional elongation (reviewed in Morales and Giordano, 2016). And indeed, CDK9 inhibition was previously found to restore DNA replication upon p53 knockdown (Yeo et al., 2016). Importantly, we observed that two different CDK9 inhibitors restored DNA replication upon depletion of Mdm2 (Fig.V.II.6A-C; Suppl. Fig.V.II.6A-C) and RNF2 (Fig. V.II.6D-F; Suppl. Fig.V.II.6D,F). Thus, ongoing transcription and perhaps the resulting R-loop formation represent a prerequisite for impaired DNA replication upon Mdm2-depletion.

A

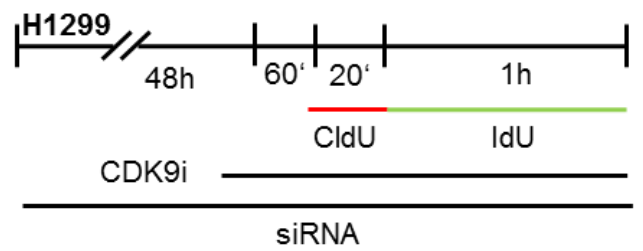

B

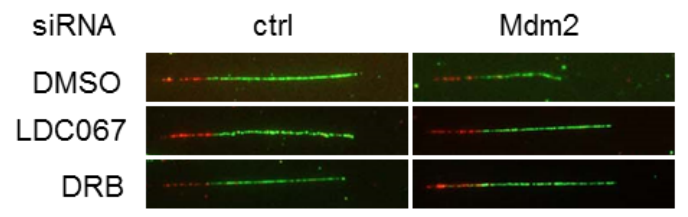

C

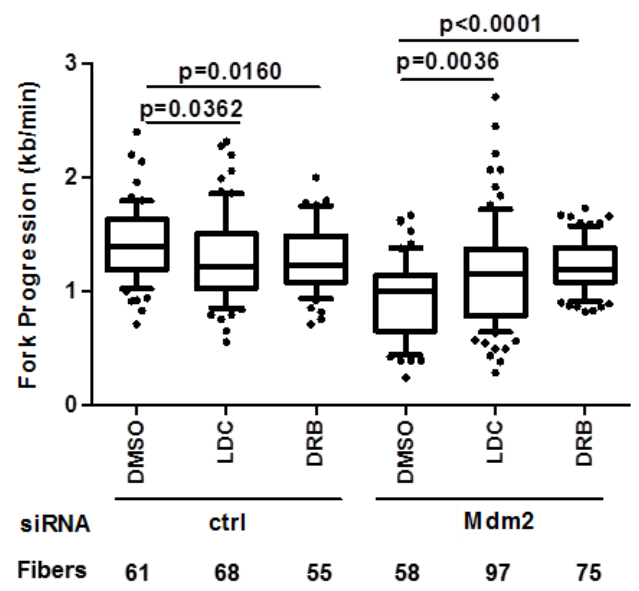

Fig. V.II.6 Rescue of DNA replication by CDK9 inhibitors upon depletion of Mdm2 or RNF2.

(A) H1299 cells were transfected with siRNA against Mdm2 for 48 hours. After 47 hours, cells are additionally treated with $10 \mu \mathrm{M}$ LDC067 and $25 \mu \mathrm{M}$ 5,6-Dichloro-1- $\beta$-D-ribofuranosylbenzimidazole (DRB), two CDK9 inhibitors, and a solvent control (DMSO). After one hour pre-incubation with the inhibitors, the cells are incubated with nucleoside analogues CldU (20 min) and IdU (60min) containing inhibitors and solvent control. (B) Representative images of labelled tracks of CldU (red) and IdU (green). (C) Fork progression analysis was carried out from IdU labelled tracks and displayed in a boxplot with 10-90 percentile whiskers. 
D

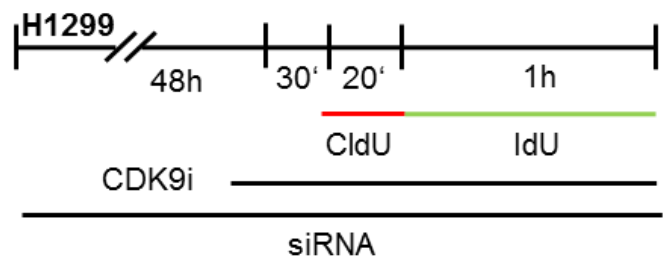

$\mathbf{E}$

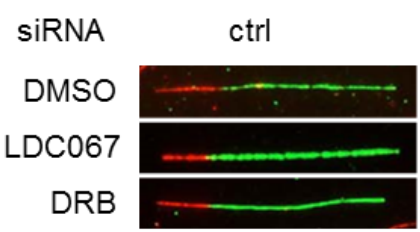

$\mathbf{F}$

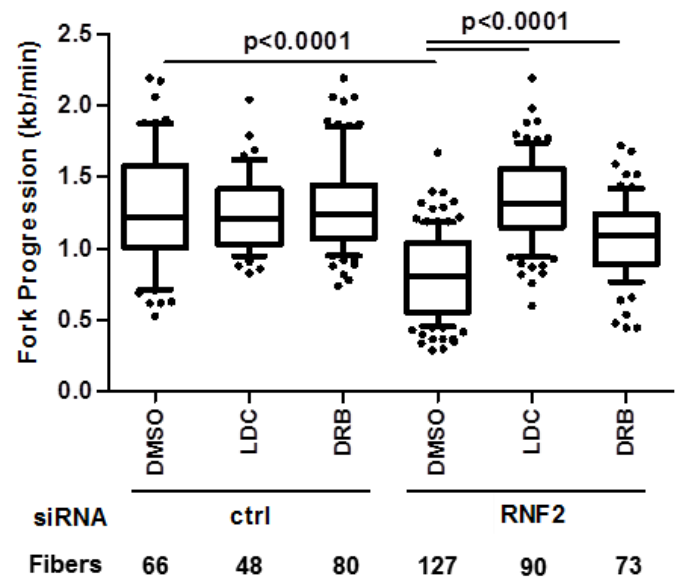

Fig.V.II.6 Rescue of DNA replication by CDK9 inhibitors upon depletion of Mdm2 or RNF2.

(D) $\mathrm{H} 1299$ cells transfected with RNF2 siRNA and pre-treated with 10 $\mu \mathrm{M}$ LDC067 and $25 \mu \mathrm{M}$ DRB for 30 minutes. Subsequently, labelling for Fiber assay was carried out as described in (A). (E) Representative images of labelled tracks of CldU (red) and IdU (green). (F) Boxplot analysis of fork progression in the IdU label.

\section{V.II.4.7 RNase $\mathrm{H}$ re-establishes DNA replication upon depletion of Mdm2 or RNF2}

Finally, we tested whether DNA:RNA hybrids in MDM2/RNF2-depleted cells represent an obstacle to DNA replication. We overexpressed RNase $\mathrm{H} 1$ to resolve hybrids that form while depleting MDM2 or RNF2. Wildtype RNaseH1 but not a catalytically inactive mutant of it gave rise to a robust rescue of DNA replication fork progression upon Mdm2 depletion (Fig.V.II.7A-D; Suppl. Fig.V.II.7A), and also upon RNF2 knockdown (Fig.V.II.7E-G; Suppl. Fig.V.II.7B-C). Taken together, these results strongly suggest that MDM2 prevents the persistence of R-loops and replicative stress. 
A

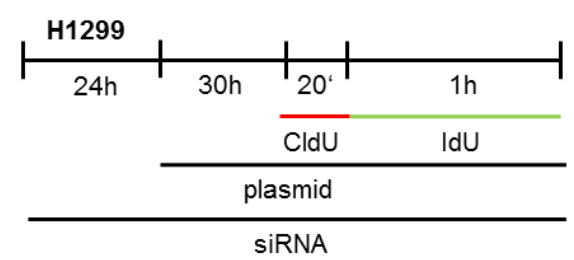

C

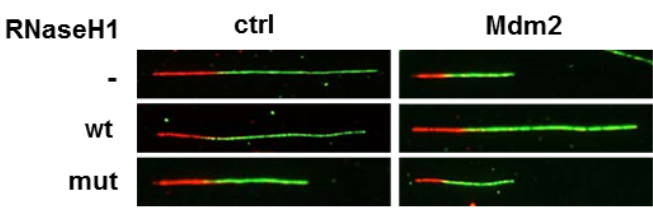

B

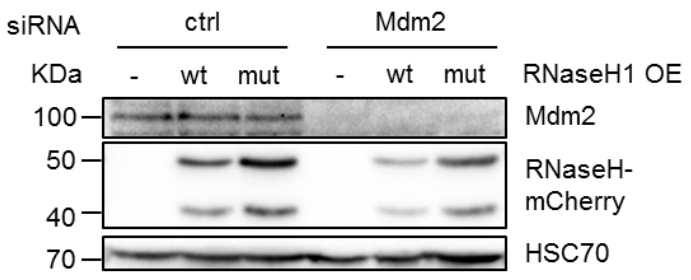

D

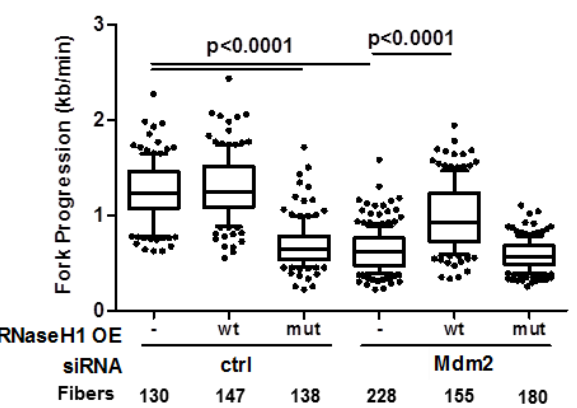

E

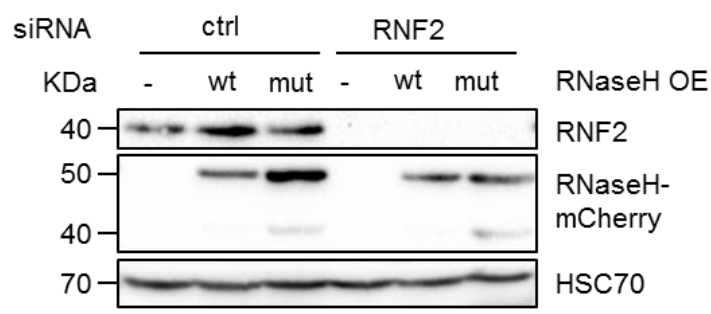

F

RNaseH1

ctrl

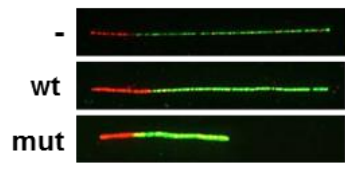

RNF2

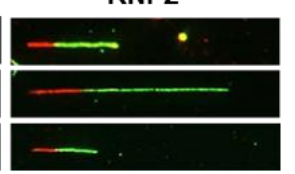

G

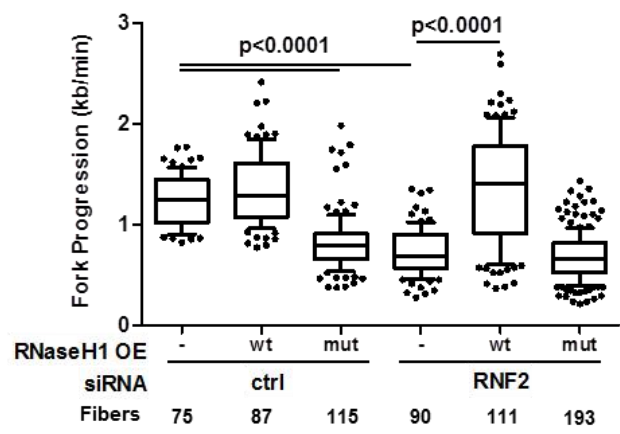

Fig. V.II.7 Restored DNA replication by removing DNA/RNA hybrids in cells depleted of Mdm2 or RNF2.

(A) H1299 cells were first transfected with siRNA targeting Mdm2/RNF2 and with a plasmid containing wildtype RNAseH1 (pICE-RNAseH1-NLS-mCherry) as well as a catalytically inactive mutant (pICE-RNASeH1D10-E48R-NLS-mCherry) and a control plasmid (pcDNA3) 24 hours after the first transfection. After another 30 hours, samples were used for fiber assay labelling by incubation with $25 \mu \mathrm{M}$ CldU (20min) and $25 \mu \mathrm{M}$ IdU (60 min). (B) Immunoblot of samples transfected as described in (A) staining for the mCherry tag of the RNAseH1 plasmids and Mdm2 to check for transfection efficiency. HSC70 is used as a loading control. (C) Representative images of immunostained tracks of CldU (red) and IdU (green) after Mdm2 depletion (Mdm2\#1) and RNAseH1 overexpression. (D) Boxplot analysis of fork progression in the IdU label of the fiber assay indicate that active RNase $\mathrm{H}$ restores DNA replication. (E) Immunoblot analysis of knockdown (RNF2) and overexpression (RNAseH1) efficiencies where HSC70 serves as a loading control. (F) Fluorescently labelled tracks of CldU (red) and IdU (green). Fork progression rates of samples depleted of RNF2 and with overexpressed RNAseH1 are shown in (G), again indicating a rescue of DNA replication. 


\section{V.II.5 Discussion}

Mdm2 and PRCs not only display similar patterns in chromatin modification and gene regulation (Wienken et al., 2016; Wienken et al., 2017) but also enable DNA replication with high processivity, independent of p53. Our results strongly suggest that these two activities are carried out through overlapping mechanisms. Both Mdm2 and PRC1/RNF2 have an E3 ubiquitin ligase function, and this sustains DNA replication. Moreover, Mdm2 and RNF2 can substitute for each other in this regard. When R-loops are removed by $\mathrm{RNase} \mathrm{H} 1$, replication is restored despite the depletion of Mdm2 or RNF2. Thus, we propose that Mdm2 and PRC1 each support DNA replication through their ability to ubiquitinate histones and prevent supraphysiological $\mathrm{R}$ loop formation.

Interestingly, members of PRC2 were found in association with DNA replication forks (Leung et al., 2013) and necessary for their symmetric progression (Piunti et al., 2014). This raises the possibility that, on top of dampening unscheduled transcription, PRC2 might directly support DNA replication, perhaps enabling immediate histone modifications simultaneously with DNA synthesis. Of note, EZH2 activity recruits Mus81 to destabilize stalled replication forks (Rondinelli et al., 2017). This might disrupt such forks on the one hand, but might enable faster resumption of replication on the other hand. It is thus conceivable that PRC2 not only dampens transcription but also alters the dynamics of DNA replication by recruiting DNA processing factors.

Curiously, the overexpression of Mdm2 has previously been found to enhance rather than diminish replication stress (Frum et al., 2014). Of note, however, Mdm2 has long been known to impair cell proliferation when overexpressed (Brown et al., 1998), whereas our study is mainly based on the depletion of endogenous Mdm2. We therefore propose that Mdm2, when present at physiological levels, actually supports DNA replication.

Depletion of Mdm2 enhances detectable DNA:RNA hybrids, and this notion might explain previously reported observations. Both p53 (Yeo et al., 2016) and Mdm2 (Conradt et al., 2013) contribute to the resistance of cells to topoisomerase inhibitors, a class of drugs that iniduces R-loops (Marinello et al., 2013). Moreover, depleting p53 sensitizes cells towards co-depletion of DHX9 (Lee and Pelletier, 2017), a helicase capable of removing R-loops (Cristini et al., 2018).

In a broader sense, RNA metabolism is becoming a major focus in current research on DNA replication stress. RNA processing factors constitute a major fraction of kinase substrates upon DNA damage (Beli et al., 2012; Matsuoka et al., 2007), making it plausible that such factors might affect DNA integrity, perhaps through R-loop formation. 
Recently, the oncogenic EWS-FLI1 fusion protein was found to cause R-loops and thereby DNA damage (Gorthi et al., 2018). Our results expand this concept, implying Mdm2 and polycomb repressors not only in the formation of a closed chromatin state, but also in the suppression of R-loops, thereby enabling proper DNA replication. A number of mechanisms were suggested for R-loops forming obstacles to DNA replication (Aguilera and Gomez-Gonzalez, 2017; Garcia-Muse and Aguilera, 2016). However, the exact nature of such conflicts between RNA:DNA hybrids and DNA replication still remains to be determined.

Besides its chromatin modifier function, MDM2 is also capable of interfering with DNA repair through binding Nbs1, one of the MRN complex members (Alt et al., 2005; Bouska et al., 2008). However, this association negatively regulates MRN-mediated repair of double stranded DNA breaks. Since Mdm2, according to our data, is a positive factor in DNA replication fork progression, we propose that this function is independent of Nbs1 binding. Formally, we cannot exclude that Mdm2 might interfere with the degradation of stalled replication forks, as has been reported for BRCA2 (Schlacher et al., 2011). However, our previous work suggested that the depletion of p53 (and thus concomitant reduction in Mdm2-levels) does not alter fork restart or reversal (Klusmann et al., 2016).

Since a minimum Mdm2 level is necessary for proper DNA replication, events that lower the amounts of intracellular Mdm2 might confer replication stress. For instance, Mdm2 levels drop in response to DNA damage, through phosphorylation and subsequent autoubiquitination of Mdm2 (Carr et al., 2016; Li and Kurokawa, 2015). This suggests that impaired DNA replication might occur in response to DNA damage, not only as a direct result of DNA lesions, but also as a consequence of Mdm2 degradation. Furthermore, most cancers show impaired p53 activity, e. g. due to p53 mutations, resulting in lowered expression of the p53-responsive gene encoding Mdm2. Thus, the generally enhanced DNA damage observed in many tumours (Bartkova et al., 2005) might at least in part result from insufficient Mdm2 levels and the resulting DNA replication stress. Taken together, Mdm2 may not only serve as a p53 antagonist, but also as an effector of p53mediated tumour suppression, modifying chromatin, avoiding R-loops and supporting replication.

On the other hand, Mdm2 represents an oncogene, and its overexpression can result from gene amplification, most notably in sarcomas. Our results suggest that not only the inactivation of p53 but also the modification of chromatin with subsequently enhanced DNA replication processivity may contribute to this oncogenicity. Importantly, Mdm2 has frequently been targeted by drug candidates, but with limited success in the clinics. To our knowledge, all Mdm2-targeting drugs that are currently evaluated in clinical studies interfere with the interaction of $\mathrm{p53}$ and Mdm2, but not with the ubiquitin ligase activity of 
the Mdm2 Ring finger domain (Burgess et al., 2016). Furthermore, our previous work indicates that the prototype drug of this kind, Nutlin-3a, does not interfere with the chromatin modifier function of Mdm2 (Wienken et al., 2016). Therefore, targeting the Ring finger domain rather than the p53 binding domain of Mdm2 can be expected to have a more profound impact on cancer cell proliferation, by targeting chromatin modification and interfering with DNA replication. This encourages the continued evaluation of Mdm2 as a drug target, regarding its various activities on p53 as well as on chromatin and on DNA replication.

\section{V.II.6 Acknowledgments}

We thank Yanping Zhang for MEFs with p53/mdm2 deletions/mutation and Pavel Janscak for providing GFP-RNAseH1 D210N cells. pCMV-MDM2 was a gift from Bert Vogelstein (Addgene plasmid \#16441), pCMV-MDM2(C464A) was a gift from Tyler Jacks (Addgene plasmid \#12086), pLenti6/V5-DEST-RNF2 was a gift from Lynda Chin (Addgene plasmid \#31216), and pICE-RNaseHI-WT-NLS-mCherry (Addgene plasmid \#60365) as well as pICE-RNaseHI-D10R-E48R-NLS-mCherry (Addgene plasmid \#60367) were gifts from Patrick Calsou. This work was supported by the Else Kröner Fresenius Stiftung, the Wilhelm Sander Stiftung, the Deutsche José Carreras Leukämie Stiftung, the Deutsche Krebshilfe, the Deutsche Forschungsgemeinschaft, the Boehringer Ingelheim Fonds (to IK). Further support came from the Swiss National Science Foundation (PP00P3_150690 to MA), the European Research Council (ERC-2016-STG 714326 DiVineGenoMe to MA), and the Forschungskredit Candoc Program of the University of Zurich (FK-16-053 to FT). IK was a member of the Göttingen Graduate School GGNB and of the IMPRS/MSc./PhD program Molecular Biology at Göttingen during this work. FT is a member of the Molecular Life Science Program of the Life Science Zurich Graduate School. 


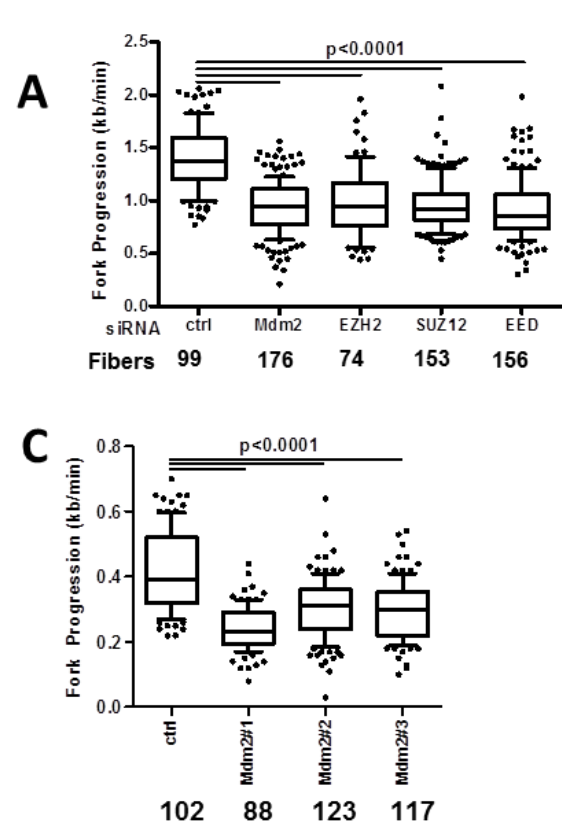

D

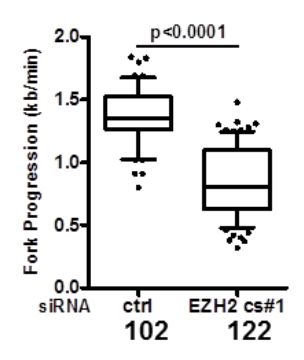

G

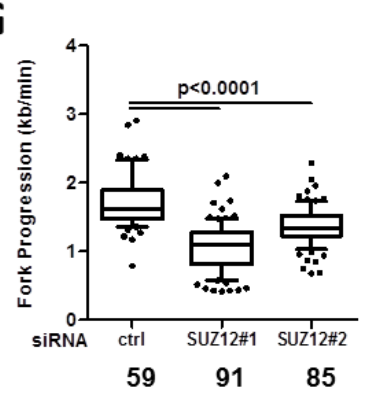

J

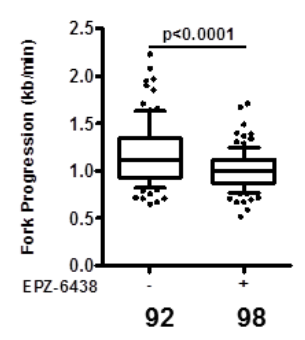

$E$

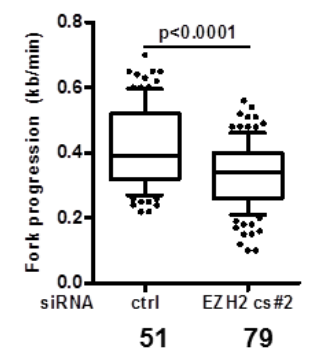

H

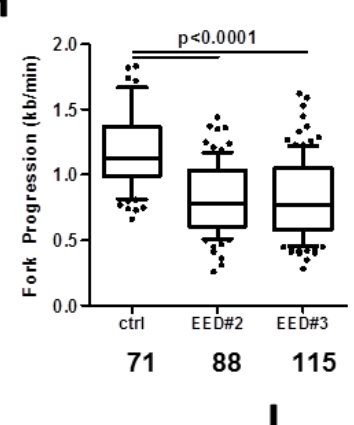

K

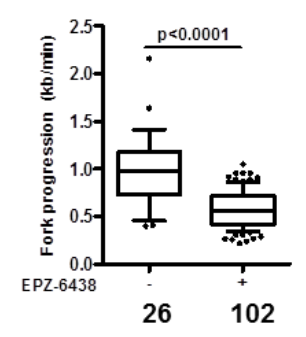

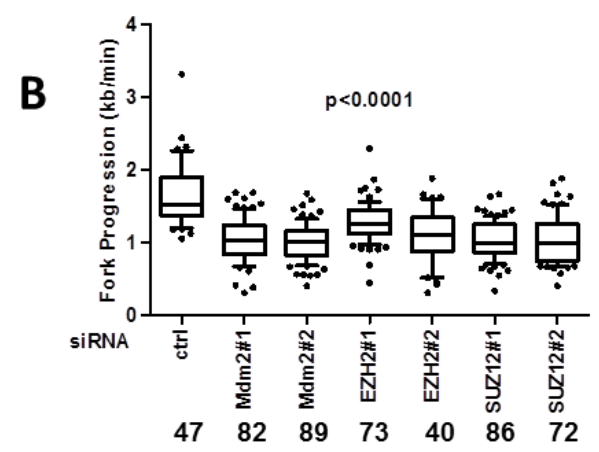

$\mathbf{F}$

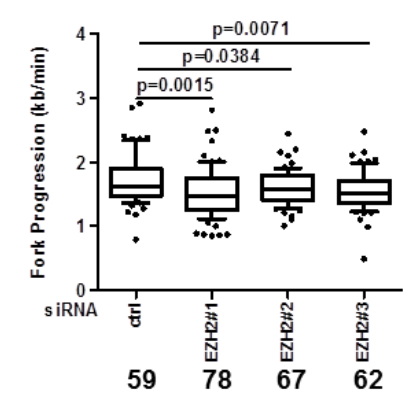

I

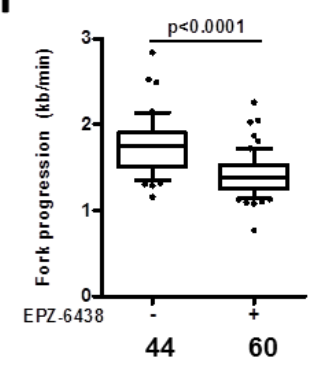

M

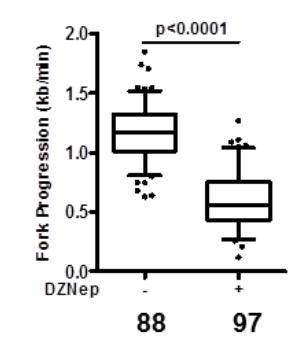

Suppl. Fig. V.II.7.1 Compromised DNA replication fork progression upon depletion of Mdm2 or PRC2 members. Related to Fig. V.II. 1. 
Suppl. Fig. V.II.7.1 continued (A) H1299 cells were transfected with a second siRNA set to Mdm2, EZH2, SUZ12, and EED for 48 hours. The experiment was carried out in parallel to the one in Fig.V.II.1D-E and therefore shares the control sample. (B) A biological replicate to the experiment displayed in Fig.V.II.1D-E and panel $A$ of this figure. Fiber analysis shows reduced fork progression after all knockdowns compared to

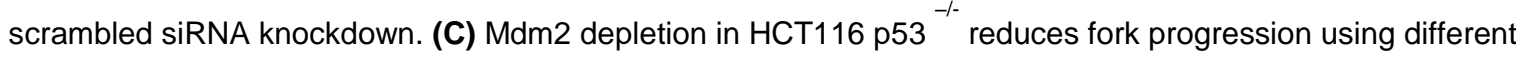
siRNAs. Depletion of PRC2 components EZH2 with two siRNAs (D-F) and SUZ12 (G) reduces fork

progression in HCT116 p53 ${ }^{-/-}$cells. (H) siRNA transfection targeting the non-catalytical PRC2 component EED for 48 hours reduces replication fork progression in $\mathrm{H} 1299$ cells. (I-J) Biological replicates to Fig. $1 \mathrm{H}$ shows reduced fork progression in $\mathrm{H} 1299$ cells treated with the EZH2 inhibitor EPZ-6438 for 48 hours. (K) A similar reduction can be observed in U2OS cells treated with the same conditions as in (G). (L-M) HCT116 p53 ${ }^{-/-}$cells treated with $5 \mu \mathrm{M}$ DZnep, another EZH2 inhibitor, for 48 hours also reduces fork progression. 
A

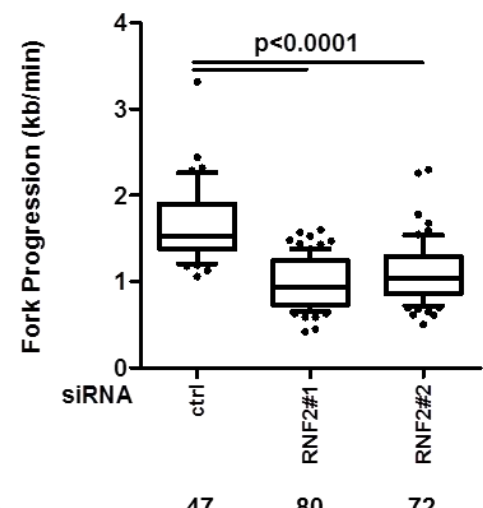

C

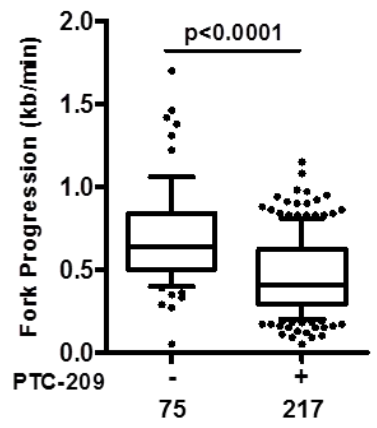

E

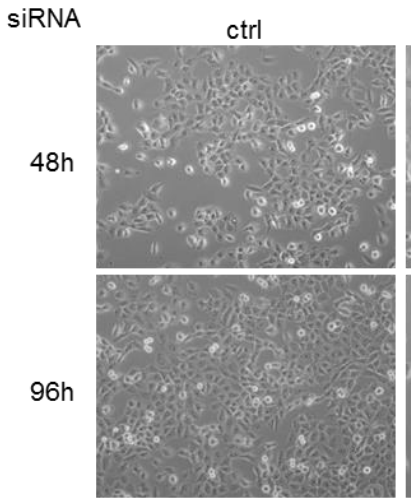

$\mathbf{F}$

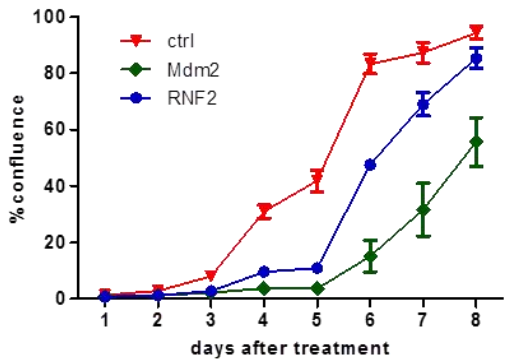

B
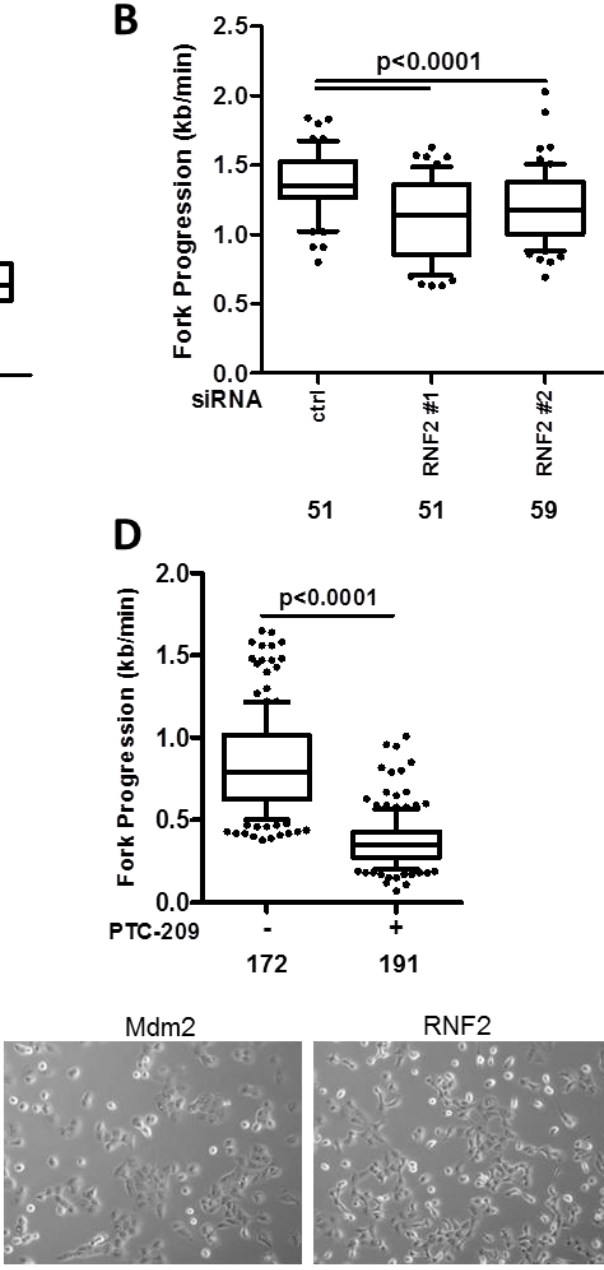

RNF2
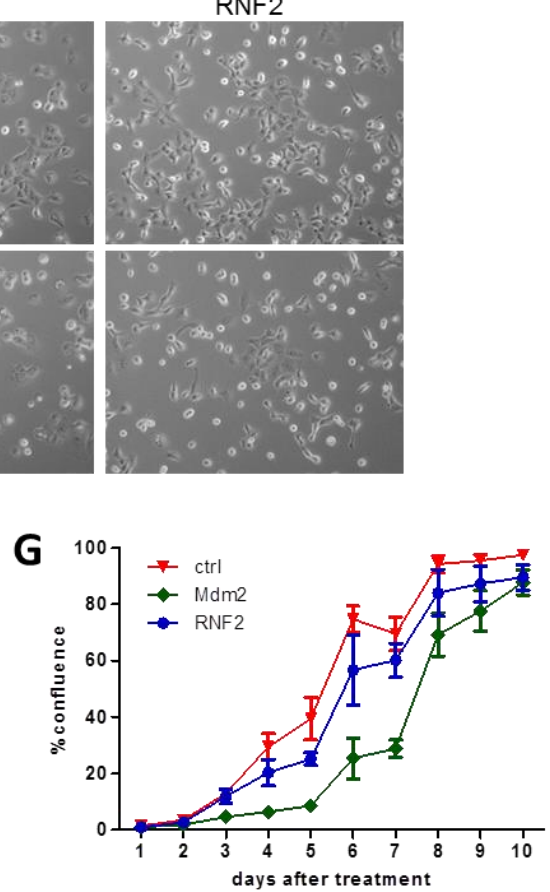

Suppl. Fig. V.II.7.2 Decreased fork progression upon RNF2 depletion or Bmi1 inhibition Related to Fig. V.II.2.

RNF2 depletion in $\mathrm{H} 1299$ cells $(\mathbf{A})$ and $\mathrm{HCT} 116 \mathrm{p} 3^{-/-}$cells (B) reduces replication fork progression in the IdU label. (C-D) Biological replicates to Fig.V.II.2G shows H1299 cells treated with $1 \mu \mathrm{M}$ of the BMI1 inhibitor PTC209 for 48 hours reduces fork progression. (E) Brightfield microscopy of $\mathrm{H} 1299$ transfected with siRNA against Mdm2 and RNF2 for 48 hours and a second transfection for another 48 hours (96h timepoint) shows reduced proliferation compared to control. (F-G) Two biological replicates of clonogenic growth assay shown in Fig. V.II.2N. 
A

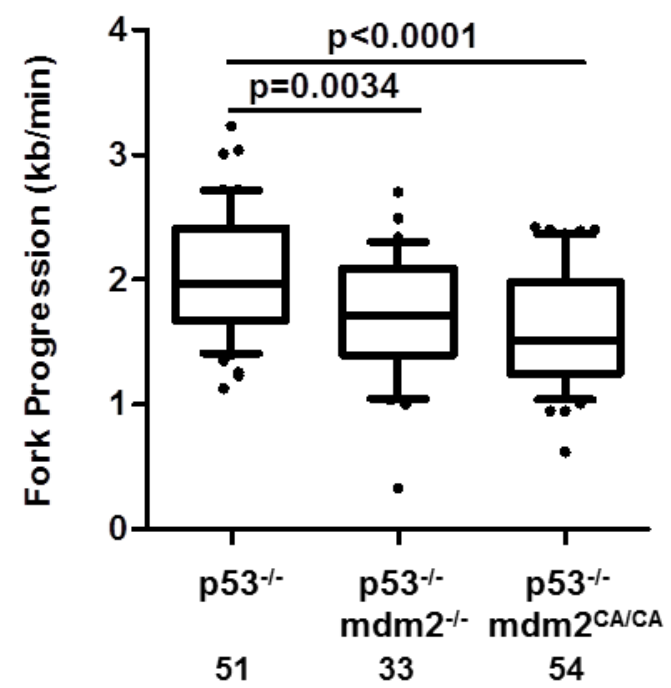

C

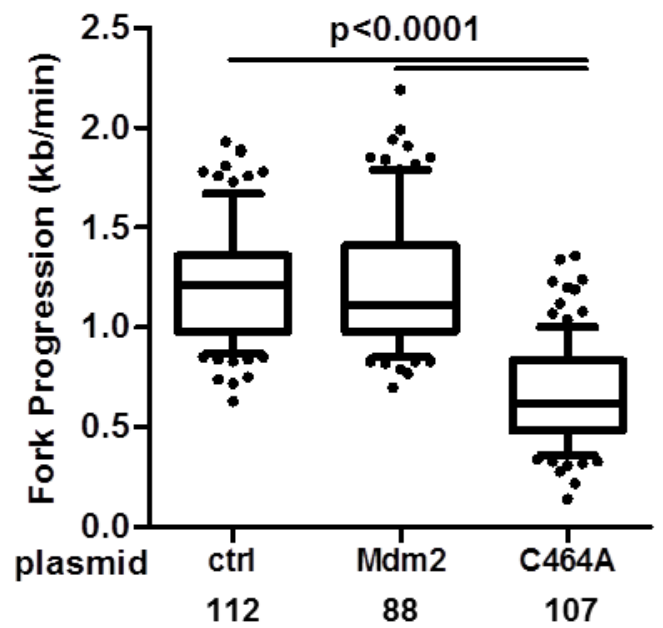

B

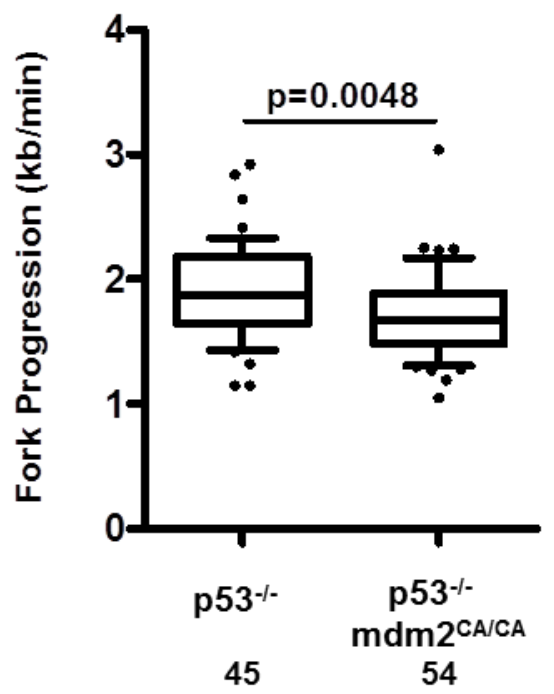

D

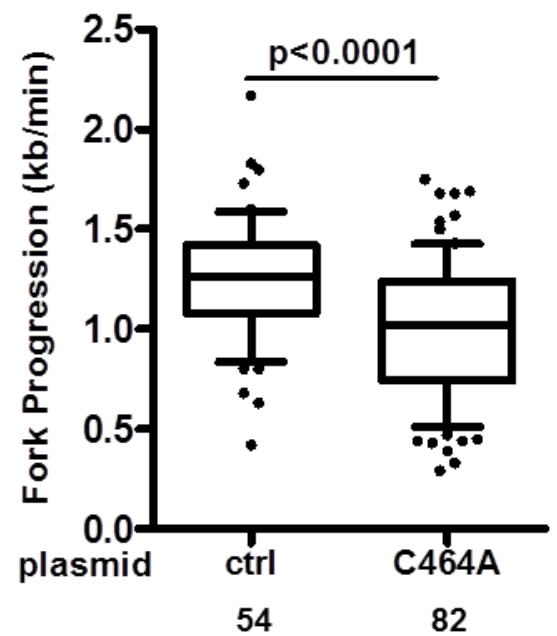

Suppl. Fig. V.II.7.3 Requirement for the Ring finger domain of Mdm2 to support replication fork progression. Related to Fig. V.Il.3.

(A-B) Biological replicates to Fig. 3B shows a reduced fork progression for p53/Mdm2 double knockout MEFs as well as a RING mutant MEF cell line with a p53-null background. (C-D) In the human H1299 cell line, an overexpression of a RING mutant Mdm2 reduces fork progression as seen in Fig.V.II.3F. 
A

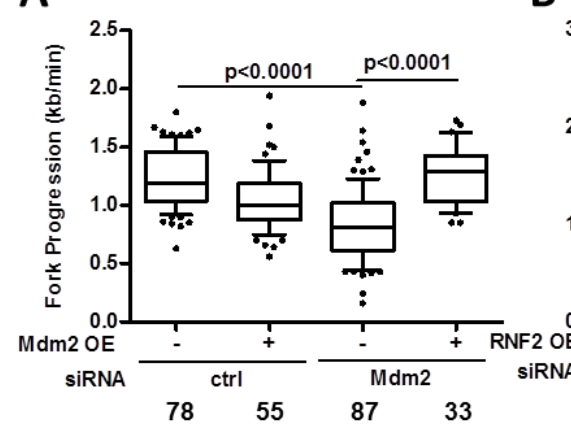

B C

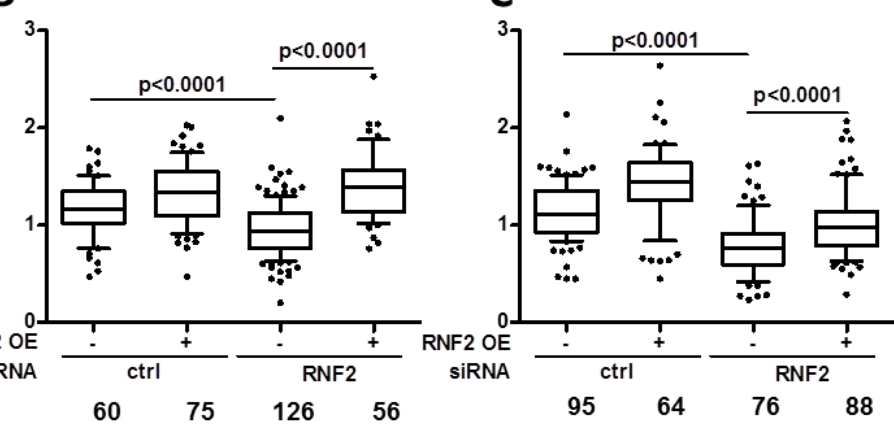

D

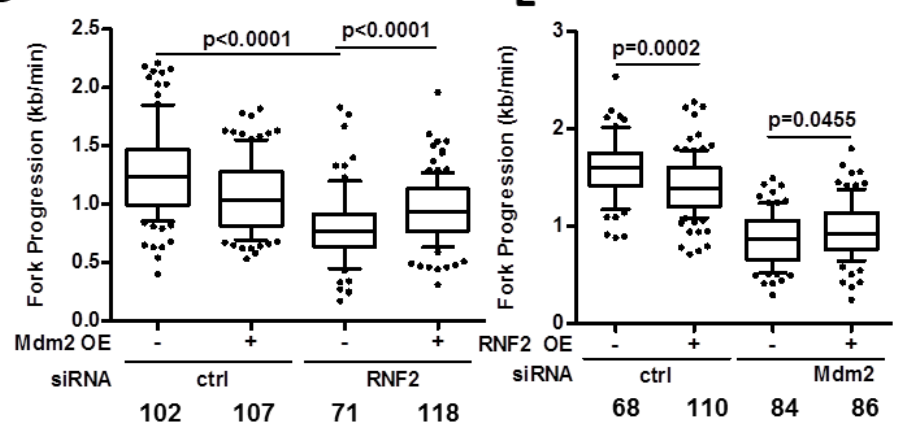

Suppl. Fig. V.II.7.4 Mutual rescue of for progression by Mdm2 and RNF2. Related to Fig. V.Il.4.

Biological replicates to Fig. 4B (A), Fig.V.II.4E (B-C), Fig.V.II 4H (D) and Fig.V.II.4K (E) show that impaired replication can be rescued by overexpression of the depleted Mdm2 and RNF2 protein as well as by the overexpression of each other in a cross-fashion. 
A

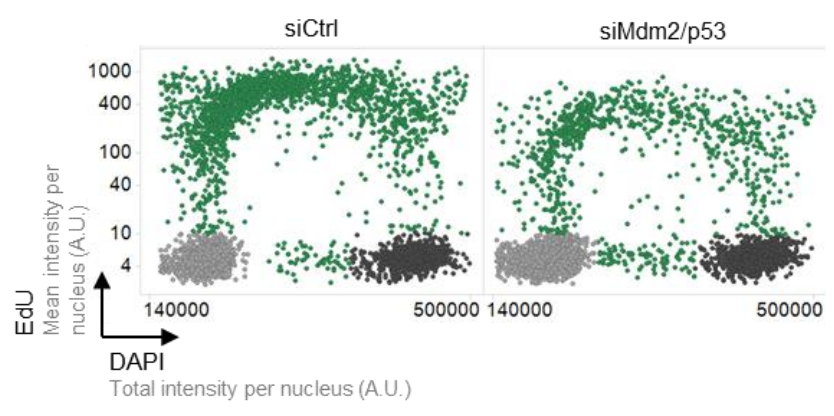

C

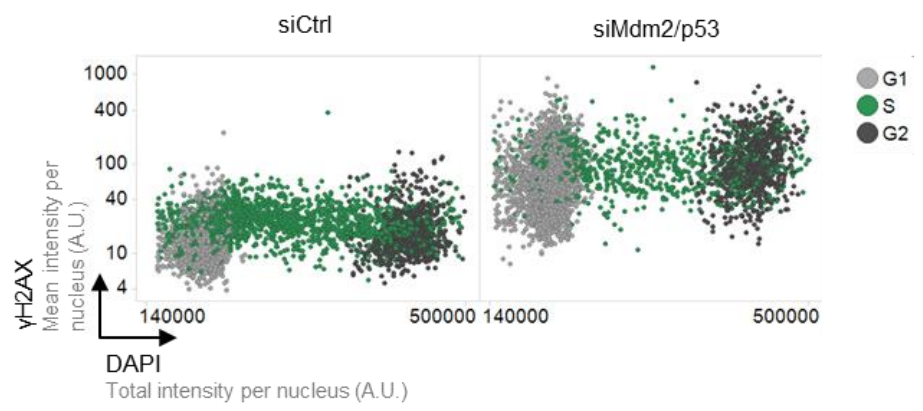

$\mathbf{E}$

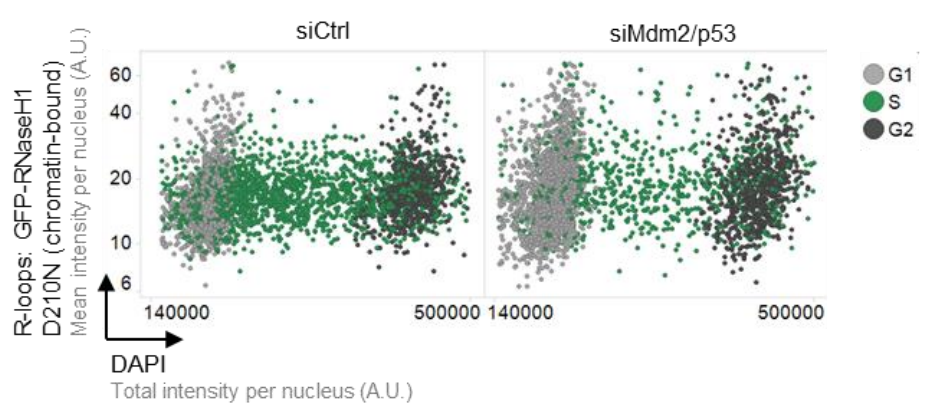

B

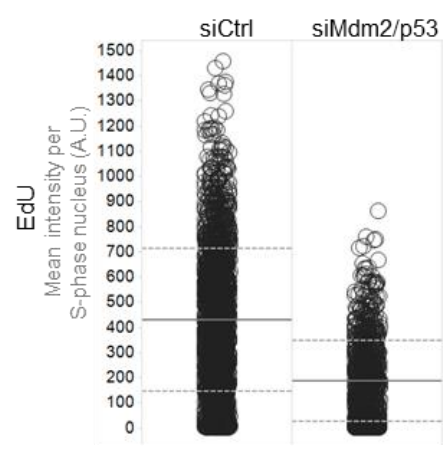

D

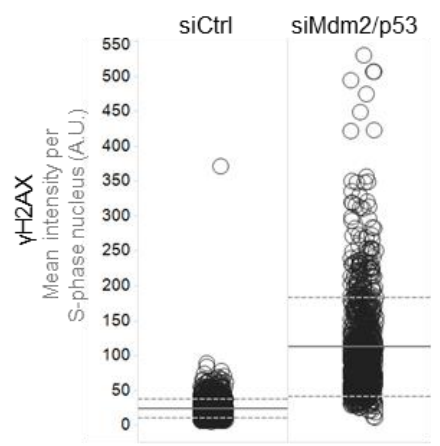

$\mathbf{F}$

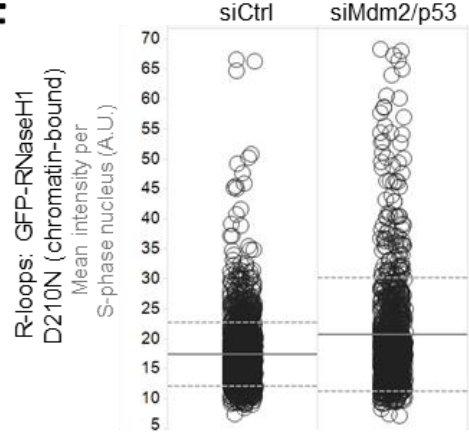

Suppl. Fig. V.II.7.5 Mdm2 depletion increases replicative stress and R-loop formation. Related to Fig. V.II.5.

As in Fig.V.II.5, U2OS cells that stably express GFP-RNaseH1-D210N were transfected with siRNA against p53 and Mdm2 for 48 hours, prior to mutant RNaseH1 induction by doxycycline treatment for another 24 hours. Next, cells were pulsed with EdU to detect DNA synthesis for 20 minutes, pre-extracted, fixed and stained and the signals for DAPI, EdU, $\mathrm{HH} 2 \mathrm{AX}$, and chromatin-bound RNaseH1-D210N were quantified by high-content microscopy. (A) Two-dimensional cell cycle staging was performed based on the EdU and DAPI signals. (B) The S-phase specific EdU signals were plotted for Mdm2/p53-depleted cells versus a control siRNA transfection. Horizontal lines represent averages and standard deviations. (C) Levels of the DNA damage marker $\mathrm{YH} 2 \mathrm{AX}$ increase upon depletion of Mdm2 and p53 throughout the cell cycle. (D) The S-phase specific $\mathrm{yH} 2 \mathrm{AX}$ signals were plotted for Mdm2/p53-depleted cells versus a control siRNA transfection. Horizontal lines represent averages and standard deviations. (E) Chromatin-bound RNaseH1-D210 marks RNA:DNA hybrids, and this signal increases when Mdm2 and p53 were depleted. (F) The S-phase specific signals of chromatin-bound RNaseH1-D210N were plotted for Mdm2/p53-depleted cells versus a control siRNA transfection. Horizontal lines represent averages and standard deviations. 

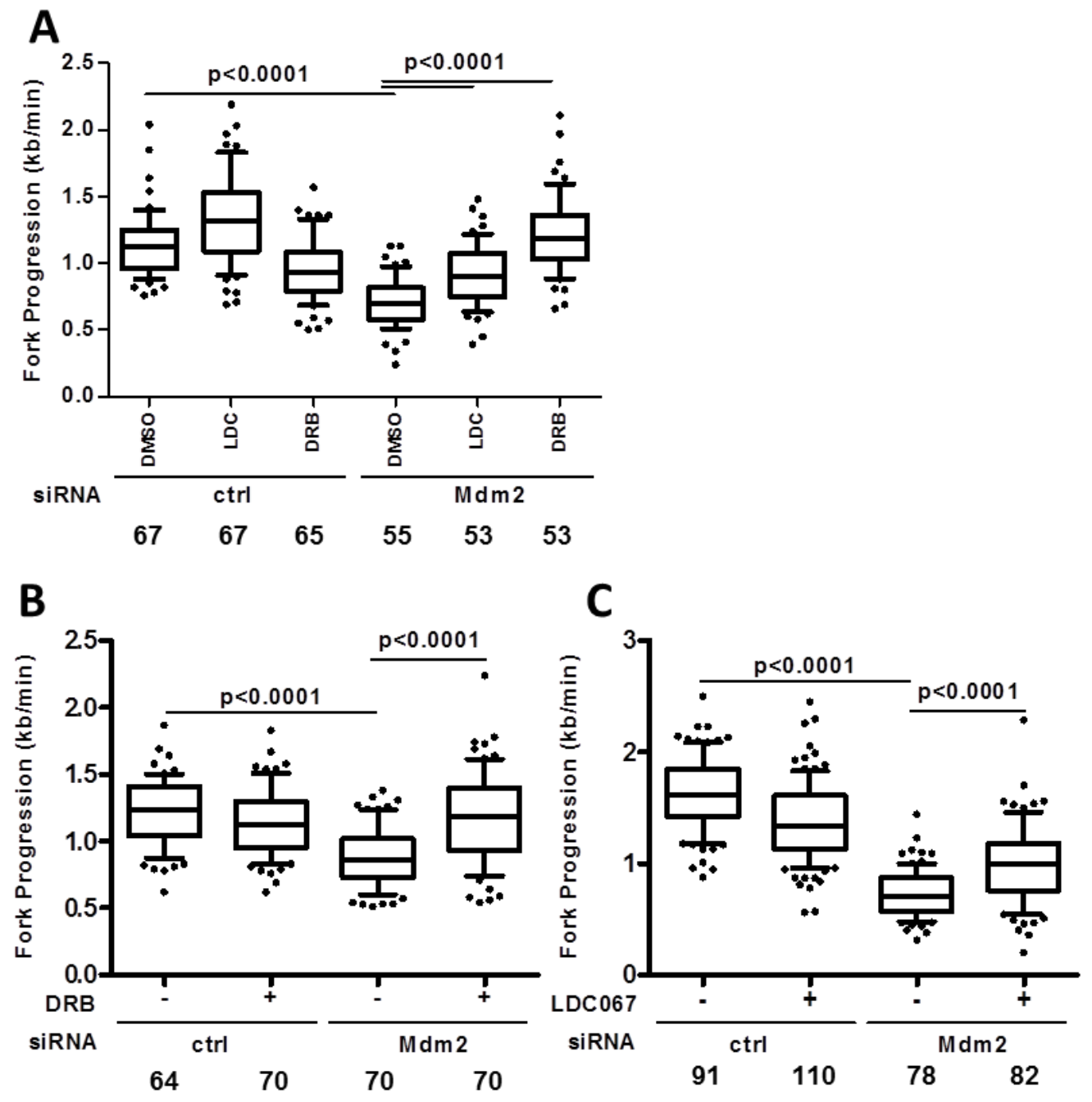

D

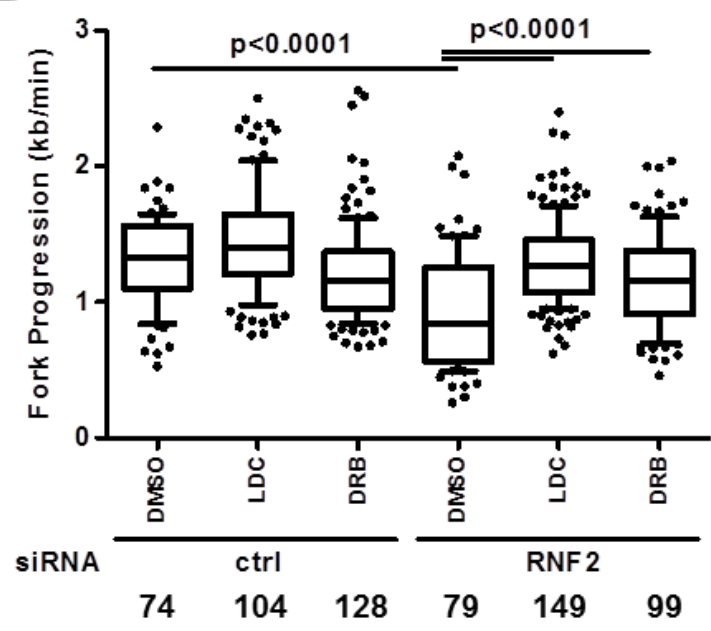

E

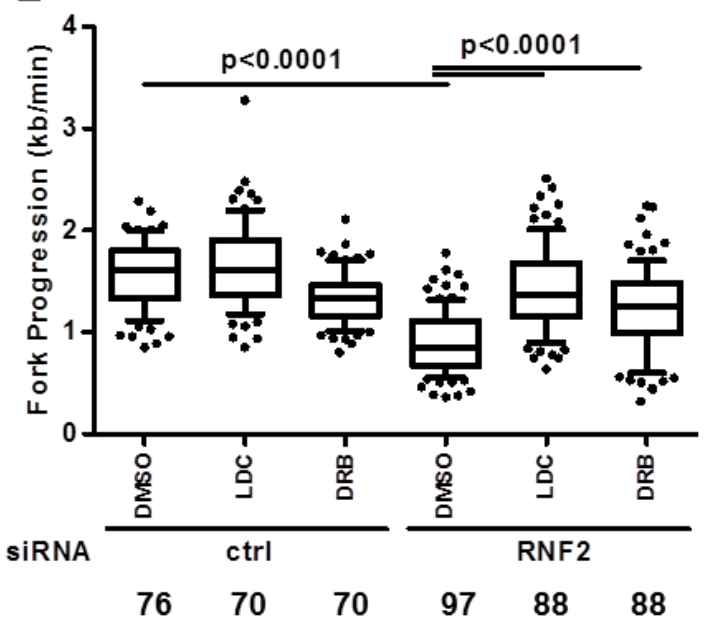

Suppl. Fig. V.II.7.6 Rescue of DNA replication by CDK9 inhibitors upon depletion of Mdm2 or RNF2. Related to Fig. V.II.6.

A decrease in fork progression caused by Mdm2 (A-C) and RNF2 (D-E) in H1299 cells can be rescued by inhibiting global transcription elongation mediated by CDK9 with two different inhibitors, DRB and LDC067. 
A

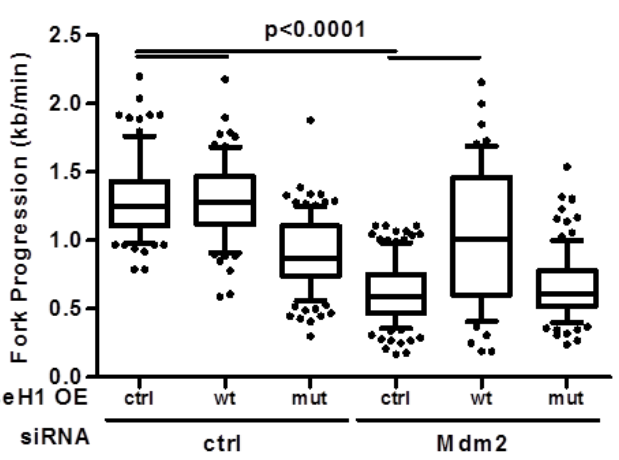

C

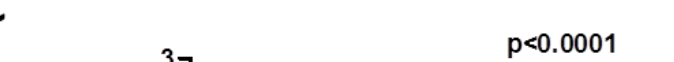

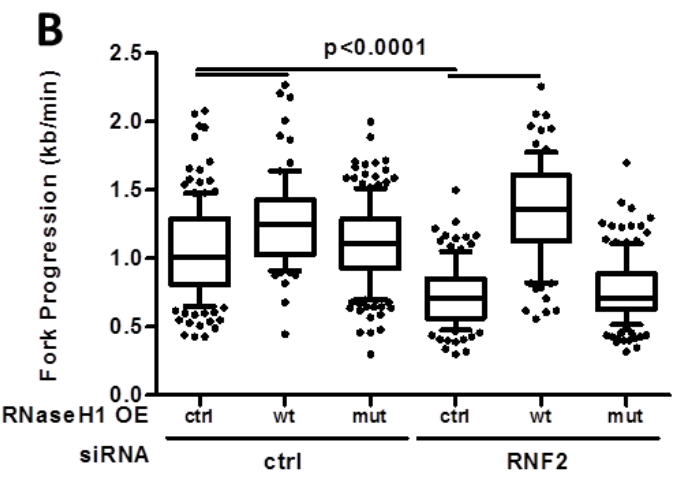

$\begin{array}{llllll}152 & 78 & 172 & 116 & 90 & 147\end{array}$

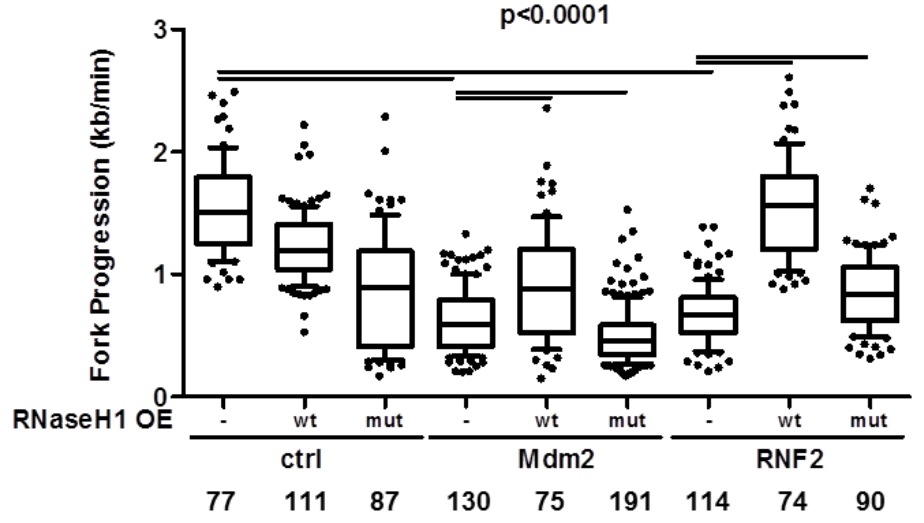

Suppl. Fig. V.II.7.7 Restored DNA replication by removing DNA/RNA hybrids in cells depleted of Mdm2 or RNF2. Related to Fig. V.II.7.

H1299 cells were transfected with siRNA against Mdm2 (A, C) and RNF2 (B, C) for 24 hours and transfected with plasmids containing wildtype and a catalytically inactive mutant version of $\mathrm{RNaseH} 1$ for another 30 hours (c.f. Fig.V.II.7A). Impaired replication fork progression displayed after both Mdm2 and RNF2 knockdown was rescued by wildtype but not mutant $\mathrm{RNaseH} 1$. 


\section{Discussion}

In this thesis, we describe a novel role of the p53-MDM2 network in supporting DNA replication to prevent genome instability. In particular, we show that p53 is required for replication processivity and its absence causes replicative stress. As a transcription factor, p53 induces the expression of its target genes to mediate its many tumour suppressive roles. MDM2, a transcriptional target of $\mathrm{p53}$, is able to support DNA replication downstream of the p53 signalling cascade. Notably, MDM2 seems to mediate these effects via its RING finger domain that has intrinsic E3 ubiquitin ligase activity used to target proteins for proteasomal degradation and for signalling purposes. The depletion of MDM2 was found to significantly increase R-loops that hinder replication forks from progressing along the DNA template. We hypothesise that MDM2, along with its interaction partner of the PRC, can prevent R-loop formation and thereby support DNA replication by mediating repressive chromatin marks on the DNA. The details of this hypothesis are discussed in more detail in the following sub-chapters. 


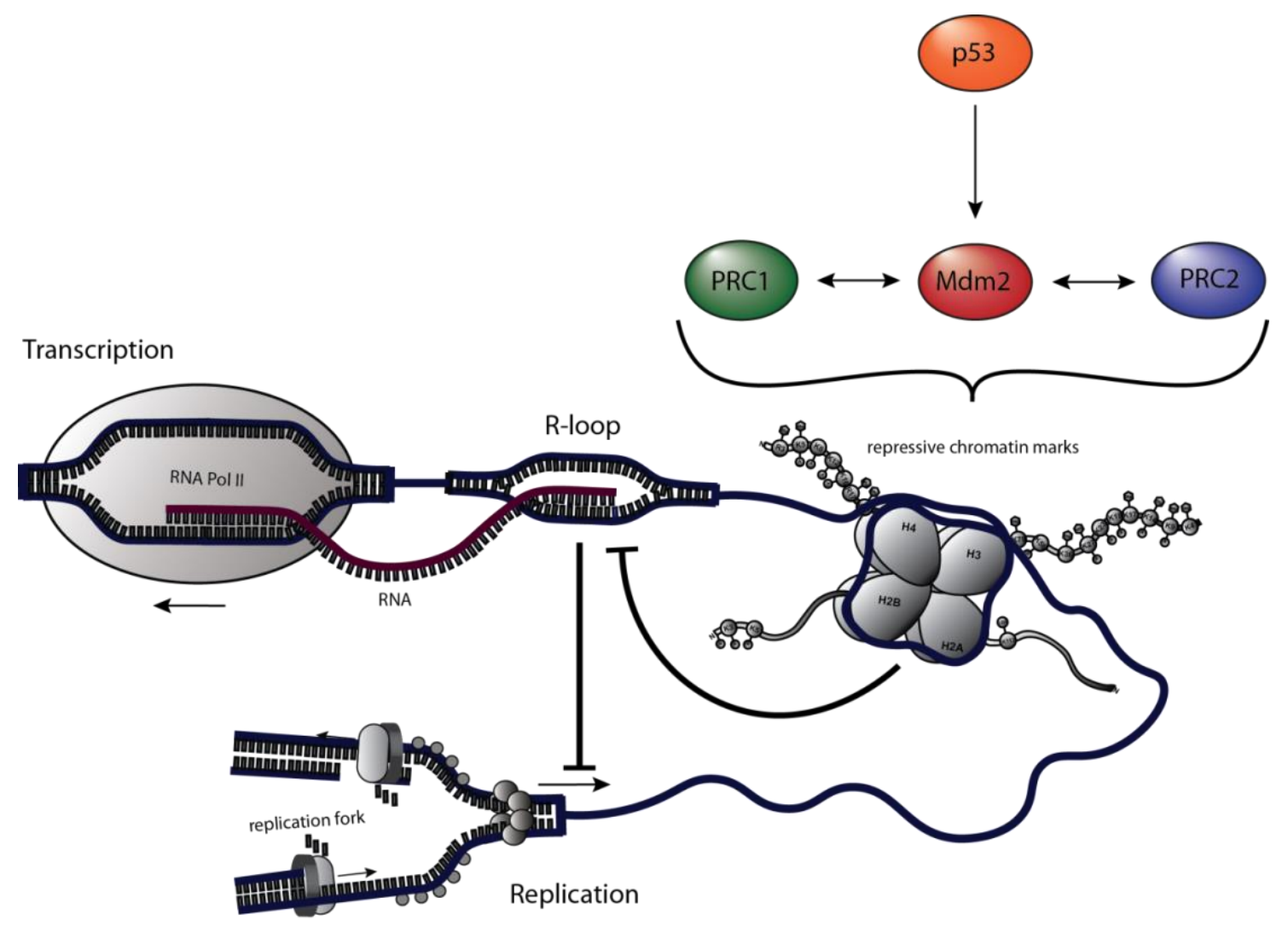

Fig.VI.1 The p53-MDM2 network facilitates replication fork progression by preventing R-loop formation.

Schematic diagram of findings described in this thesis. p53 supports DNA replication fork processivity in a number of primary and tumour cell types. We found that these effects are not directly carried out by p53 but rather induced by its activity as transcription factor responsible for target gene expression. Downstream of p53, MDM2 acts to repress target gene expression in conjunction with Polycomb repressor complexes 1 and 2 by depositing histone marks. In the absence of MDM2 and PRC components, R-loops accumulate and hinder replication forks from progressing along the DNA template causing replicative stress and DNA damage signalling. 


\section{1 Non-canonical roles of p53 in tumour suppression}

Tumour suppression by p53 was long thought to be a result of acute DNA damage responses including cell cycle arrest and repair. However, recent evidence suggests that p53 is indeed important for tumour suppression but its response to acute genotoxic stress is dispensable for this. Experimental evidence comes from knock-in mouse models carrying inactive TADs, revealing that losing the $p 53^{25,26}$ TAD1 impairs the ability to mediate cell cycle arrest and apoptosis but is still sufficient for tumour suppression, whereas a loss of both TADs in the p53 $3^{25,26,53,54}$ mutant also impairs all three activities (Brady et al., 2011). A similar study by Li et al. demonstrated that p53 was still able to suppress tumour formation in mice when its DNA binding domain was mutated (Li et al., 2012). In addition, mice deficient for key effectors of the DNA damage response downstream of p53 (e.g. cdkn1a, puma, noxa) were not more prone to tumour formation than wildtype littermates (Valente et al., 2013). Taken together, this data highlights the importance of p53 in tumour suppression but suggests the likelihood of alternative pathways by which $p 53$ achieves this.

Various non-canonical pathways for p53-mediated tumour suppression have been described in recent years (Mello and Attardi, 2018). Our work and others identified a role of p53 in maintaining genome integrity that further adds to its role as the "guardian of the genome" (Lane, 1992). This is achieved by transcriptional activation of target genes involved in DNA repair and by supporting DNA replication as well as restricting retrotransposon activity (Klusmann et al., 2016; Sengupta and Harris, 2005; Wylie et al., 2016; Yeo et al., 2016). Other studies have also attributed the title "guardian of the epigenome" to p53 as it seems to keep DNA methylation in check (Tovy et al., 2017). Furthermore, p53 can also induce metabolic changes via non-canonical pathway. p53 inhibits both glycolysis and autophagy, processes involved in energy supply and the removal of damaged cellular components that are essential for tumour suppression (Kenzelmann Broz et al., 2013; Kruiswijk et al., 2015). In addition, p53 also inhibits stemness, promotes differentiation and is thought to regulate cell migration and invasion (Krizhanovsky and Lowe, 2009; Muller et al., 2011). Among others, these non-canonical roles of p53 contribute to its tumour suppressor activity but exactly how all these roles come together in a cell and whether they are activated in a cell type-/ or developmentspecific manner remains to be elucidated. 


\section{VI.1.1 The non-canonical guardian of the genome supports DNA replication}

Maintaining genome integrity is key to tumour suppression and seems to be mediated by p53 in a variety of ways. Next to the canonical pathways of the DNA damage response and checkpoints, we have identified a novel role of p53 in protecting a cell from endogenous DNA damage during replication. The presence of wildtype p53 in a cell supports DNA replication fork processivity that leads to a higher fork progression rate and a lower incidence of both fork stalling and extra origin firing (Fig.V.I.2). Manipulating the levels of p53 in a cell also modulates fork progression rates - an accumulation of p53 increases it, whereas a depletion of p53 reduces fork progression. As is the case for most other roles of p53, supporting DNA replication does not seem to be a direct effect of p53 but rather mediated via its transactivation of target genes. One argument for this is the delayed effect of p53 accumulation on fork progression. A three-hour treatment with the MDM2 inhibitor Nutlin-3a was not sufficient to affect replication even though p53 levels were elevated. In contrast, a longer incubation of cells with Nutlin-3a not only accumulated levels of p53 but also showed an increase in target gene expression, exemplified by p21 and MDM2, and in this time was able to increase replication fork progression significantly (Fig.V.I.2A-D, Suppl. Fig.V.I.2C-F). The fact that fork progression was not only modulated by 553 levels in U2OS cells that harbour high intrinsic replicative stress but also in primary non-transformed cells argues for the presence of a general role of p53 in supporting DNA replication that becomes even more important under replicative stress conditions found during tumourigenesis.

\section{VI.1.2 A novel strategy to distinguish between fork velocity and fork processivity}

In our study, we attempted to distinguish between replication fork velocity and processivity, both of which affect the length of the replicated tracks observed and thus the amount of under-replicated DNA and genome instability. Conventional fiber analysis solely assesses the length of two different labels in a given time and cannot distinguish between velocity and processivity of the DNA polymerase. The readout of a slow and a prematurely stalled replisome is the same - a short fiber and a low fork progression rate (Fig.VI.1.). 

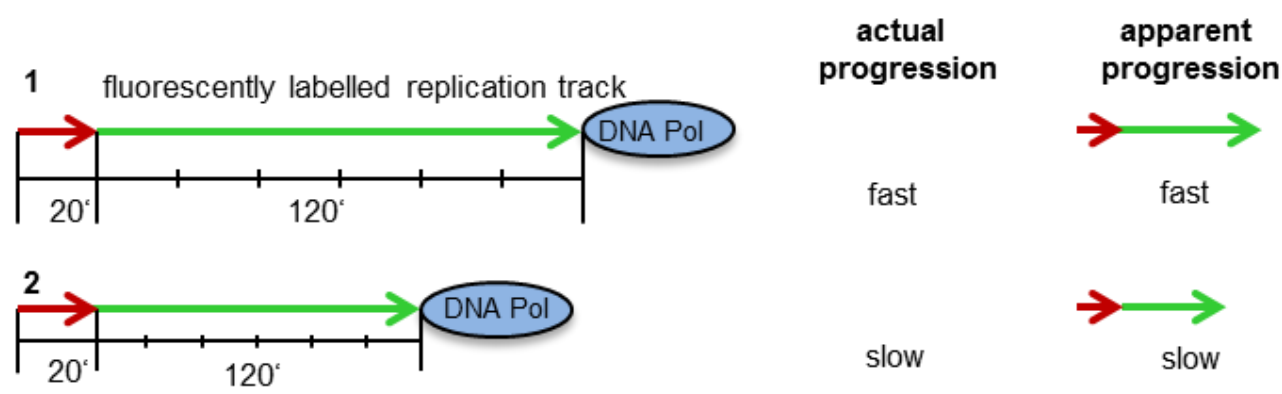

slow
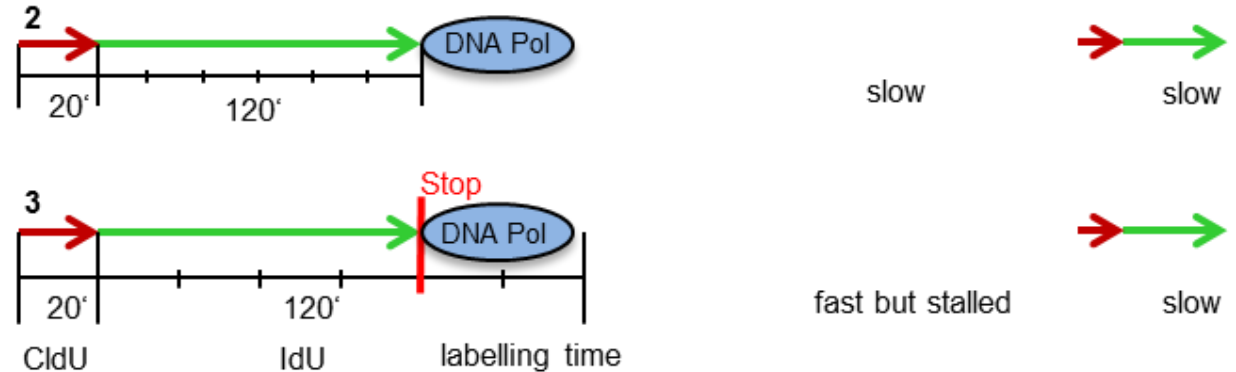

Fig.VI.1 Fiber length is determined by both fork progression and processivity. cf. Suppl. Fig. V.I.7.3E

Fiber length measured from labelled tracks in a conventional two-label Fiber assay is affected by both speed and processivity of the replication fork. (1) Fast polymerase movement allows more nucleotides to be incorporated, thus a longer labelled stretch appears. (2) Slow progression of the replication machinery results in shorter labelled tracks. (3) Fast but non-processive movement of replication also results in shorter labelled tracks as the label is not incorporated for the entire labelling time.

Conventional analysis of fork stalling involves the quantification of fibers with only the first label (red) incorporated. Mostly, the presence of red-only structures is accompanied by increased origin firing to compensate for fork stalling and can be analysed by quantifying green-red-green structures representing bidirectional first label origins. Quantification of these types of structures can provide an estimate of the amount of fork stalling and origin firing present in a sample but is not particularly accurate as these structures can also arise from different scenarios including replication termination that is not due to obstacles on the template but rather the end of a replicon.

To overcome limitations of the conventional assay, we developed a novel fiber assay strategy to distinguish between effects on fork velocity and processivity (Fig.V.I.2F-J; Fig.V.II.2I-M). Analysis with both the conventional and the novel "fork stalling fiber assay" revealed that p53 affects replication by supporting fork processivity rather than by increasing fork velocity. This effect was additionally accompanied by reduced origin firing seen in the conventional fiber assay. This complementing data suggests that the fork stalling fiber assay indeed works to dissect effects observed in the conventional fiber assay into fork velocity and processivity and can be used as an additional tool in future studies. However, a limitation of this method is the high number of samples that need to be analysed in order to obtain sufficient data and is therefore more suitable for providing additional information rather than for standard use in replication studies. 
An ongoing replication fork can be affected in many ways. On one hand the replisome itself can be altered by post-translational modifications and interaction partners to increase its association with the template DNA. Alternatively, the removal of obstacles along the DNA template such as proteins or secondary structures can also increase fork progression. Lastly, optimising the availability of building blocks in form of dNTPs would also be able to increase fork progression. As we only observe an effect of p53 on replication fork processivity and not velocity, the latter seems less likely to affect only one and not both parameters. Thus, a direct modification of the replisome or the loss of impediments on the DNA template seemed to be possible mechanisms by which p53 supports replication fork processivity. 


\section{VI.2 Changes in the chromatin landscape can affect replication fork progression}

The delayed effect of p53 accumulation to support DNA replication observed with our data (Fig.V.I.2B-D, Suppl. Fig.V.I.2C-F), argues for the involvement of transcriptional target genes of p53, many of which take more than six hours to be induced upon p53 activation (Allen et al., 2014). Taking into account that replication can be affected by the presence of secondary structures or proteins bound to the DNA template, we focussed on MDM2, a p53 target gene shown to modify chromatin compaction. Independent of p53, MDM2 associates with the Polycomb repressor complexes via its amino terminal domain at respective target genes sites to enhance the placement of the repressive chromatin marks H3K27me3 and H2AK119ub1 (Wienken et al., 2016).

MDM2 is the most important negative regulator of p53 but also its transcriptional target, forming a negative feedback loop between the two proteins that helps to keep p53 levels under tight control. In order to dissect the mechanism downstream of p53 more accurately, studies on the effect of MDM2 on replication had to be conducted in a p53-null background. Strikingly, the depletion of MDM2 in cells that had no functional p53, further impaired their replication, strongly suggesting that MDM2 acts to support DNA replication downstream of p53 (Fig.V.I.3H-J, V.I.4J-L, V.II.1C-E). Similarly, a disruption of the Polycomb repressor complexes 1 and 2 by siRNAs and pharmacological inhibitors targeting catalytic as well as accessory components, caused a significant decrease in fork progression rates (Fig. V.II.1C-I, 2B-G). This complements a previous studies showing an impaired replication fork progression after EZH2 (PRC2) depletion in association with decreased inter-origin distance in H-RAS transformed primary cells (Piunti et al., 2014).

Common to both MDM2 and the PRCs is their ability to induce histone modifications to reversibly silence target genes in the form of facultative heterochromatin. The presence of facultative heterochromatin is closely linked to replication timing as it blocks the formation of pre-RCs (Trojer and Reinberg, 2007; Zofall et al., 2016). A study on the repressive H3K9me3 mark recently showed that a histone demethylase is required for these latereplicating regions to be accessible for pre-RC formation (Wu et al., 2017). By depleting MDM2 and PRC components, target genes that would have otherwise been marked as late-replicating will no longer be repressed by chromatin compaction and their open chromatin structure would allow for the formation of pre-RCs earlier in S-phase. It is important to note that histone marks are stable and only newly deposited histones will be affected by a loss of MDM2/PRCs and the H3K27me3 and H2AK119ub1 marks mediated 
by them. Thus, an effect on replication can only be observed after cells have progressed through at least one cell cycle. This fact matches our observations that reduced fork progression can only be observed after 48 hours of depleting EZH2 activity with EPZ and DZNep (Fig.V.II.1F-H, Suppl. Fig.V.II.1I-M).

\section{VI.2.1 MDM2 and Polycomb repressor complexes - similarities and differences}

Even though both Polycomb repressor complexes mediate histone modifications that are thought to promote facultative heterochromatin formation, the nature of the modifications and the enzymes responsible for them are very different. EZH2, the catalytic subunit of the PRC2 contains a SET domain with histone-methyltransferase activity. Next to the socalled "writers" that add modifications to histone tails, "erasers" in the form of demethylases remove them, and "readers" contain domains that specifically detect and bind to methylated tails. Readers often act as adaptor molecules to bridge the signal on the histone tail to a cellular response. One such reader mechanism was recently shown to occur at replication forks, too. In this study, EZH2 was shown to deposit H3K27me3 marks at stalled replication forks as a writer to recruit the MUS81 endonuclease to sites of damage. This in turn allows homologous recombination-mediated repair of stalled fork to preserve genome stability (Rondinelli et al., 2017). According to this model, a depletion of MDM2 and PRC2 components in our cells would lead to impaired H3K27me3 deposition and recruitment of repair enzymes at stalled replication forks followed by genomic instability. If true, this would be visible in the form of shorter fibers and increased fork stalling events that cannot be rescued by repair, both of which match our data.

In contrast, the PRC1 complex contains RNF2 as its catalytic core to ubiquitinate $\mathrm{H} 2 \mathrm{~A}$ at lysine 119. Like MDM2, RNF2 contains a RING domain with intrinsic E3 ubiquitin ligase activity. The finding that both MDM2 and RNF2 expression can also rescue replication defects caused by the depletion of each other respectively, argues for an independent but compensatory mechanism of the two E3 ligases (Fig.V.II4H-M). By using catalytically inactive RING mutants of MDM2 we identified this domain to be critical in supporting DNA replication (Fig. V.II.3A-G).

Previous work identified a physical interaction between MDM2 and both Polycomb repressor complexes with a co-localisation of these complexes at transcriptional start sites of common target genes (Wen et al., 2014; Wienken et al., 2016). Another study identified an interaction of MDM2 with two other methyltransferases, SUV39H1 and EMHT1, via the 
acidic domain of MDM2 (Chen et al., 2010). Both mediate di- and trimethylation of H3K9, which is a mark also associated with heterochromatin and late replicating origins (Becker et al., 2016; Rivera et al., 2014). It remains unclear whether MDM2 catalyses the two PRC-mediated chromatin marks directly, or if enhances the activity of the PRC complexes or both. It seems possible that it interacts with a number of histone methyltransferases, including $\mathrm{EZH} 2$, to target them to specific sites and enhance their activity through the stimulation of its intrinsic E3 ubiquitin ligase activity towards local H2AK119 in conjunction with PRC1, but further studies would be required need to clarify its exact role in this process.

\section{VI.2.2 MDM2 and MDMX}

MDMX is a homologue of MDM2 that arose from a gene duplication about 440 million years ago. Even though they share the same domain architecture and differ in length by just one amino acid, their activities differ. The RING domain that both proteins contain in their C-terminal half seems to be catalytically inactive as a E3 ligase in MDMX, but is required to form heterodimers with MDM2 (Shvarts et al., 1996; Tanimura et al., 1999). As with MDM2, the existence for p53-independent roles of MDMX have been postulated as a result of the observation that p53-mutant cancers often concomitantly lose functional MDMX (Bartel et al., 2005; Carrillo et al., 2014). An alternatively spliced variant of MDMX lacking exon 6 produces a truncated version of the transcript that consists of a p53binding domain only. This isoform, also referred to as MDMX-S, lacks the RING portion of the protein required for MDM2 interactions and is associated with a poor prognosis even in the absence of wildtype p53 (Lenos et al., 2012). In addition, it was recently shown that the $\mathrm{Zn}$-finger domain in MDMX is able to maintain genome stability by preventing multipolar mitosis whereas its RING domain is able to suppress proliferation in the absence of p53 (Matijasevic et al., 2016). Taken together, the data discussed here argues for a p53- and possibly even MDM2-independent role of MDMX in preventing genome instability. As its RING domain has been described to regulate proliferation independently of p53, it would be of interest to investigate whether the proliferation defect is due to impaired replication and whether this is dependent on a MDM2 interaction and its intrinsic E3 ligase activity. 


\section{VI.3 R-loops form in the absence of MDM2}

Apart from proteins bound to chromatin, secondary structure elements can pose as obstacles to a moving replication fork. One example of such a structural element described to have detrimental effects on replication are stable DNA:RNA hybrid structures called R-loops. The mechanism by which R-loops form is not completely understood but experimental evidence suggests that they can arise in a co-transcriptional fashion in the so called "thread-back model" (Fig.II.3.5). In such a scenario, the DNA and RNA strands that exit the RNA polymerase complex via two separate exit channels re-anneal at complementary sites so that the second DNA strand is displaced (Santos-Pereira and Aguilera, 2015; Westover, 2004).

We observed an increase in chromatin-bound R-loops in p53- and MDM2 co-depleted cells. The method used to analyse this was developed and carried out by the Altmeyer group in Zurich and involves an inducible cell system for overexpressing catalytically inactive GFP-tagged RNaseH1 (Teloni et al., manuscript submitted). This enzyme is one of several players that can bind to remove R-loops in a cell by removing the RNA moiety of the hybrid structure (Wahba et al., 2011). A point mutation in its catalytic triad render its ribonuclease activity inactive but retains its ability to specifically bind to R-loops. A GFPtag on the mutant $\mathrm{RNaseH} 1$ allows a microscopy-based detection and quantification of the chromatin-bound fraction of the protein after permeabilisation of the cells. According to the high-throughput analysis of data generated in this cell system, replicative stress and Rloops are present in cells lacking both p53 and MDM2 in all phases of the cell cycle including S-phase and could explain why we see impaired replication fork progression when depleting MDM2 in a p53-null background.

But how exactly can the depletion of a protein involved in chromatin compaction lead to the re-annealing of nascent RNA to its template DNA strand and how does that affect DNA replication?

\section{VI.3.1 R-loops - natural intermediates and drivers of genome instability}

R-loops are natural intermediates in a number of physiological processes including Ig class switch recombination, bacterial DNA replication, mitochondrial DNA replication, as well as the regulation of eukaryotic transcription (Aguilera and García-Muse, 2012). Genome-wide analysis has revealed a preferential formation of R-loops at CpG-island promotor sites that have an asymmetric arrangement of guanosines and cytosines called 
a "GC-skew", and form stable G-quadruplex structures (Sollier and Cimprich, 2015). As Rloops protect the template DNA from de novo methylation, these promotor regions can no longer be epigenetically silenced, thus gene expression at these sites is active (Ginno et al., 2012). At transcription termination sites, G-rich stretches downstream of the poly-A signal allow the formation of R-loops, associated also with low DNA methylation patterns, and RNA polymerase II pausing upon an encounter with the hybrid. Helicases then catalyse the removal of the R-loop structure and facilitate the release of the RNA molecule for efficient transcription termination (Santos-Pereira and Aguilera, 2015).

As intermediates, R-loops have fast turnover rates in order to regulate physiological processes efficiently. It might be this turnover rate that causes the shift from a regulatory $\mathrm{R}$-loop to a persistent $\mathrm{R}$-loop that is a threat to genome integrity.

The DNA:RNA hybrid within a R-loop is thermodynamically very stable and difficult to access, whereas the single stranded portion of the displaced DNA is very vulnerable to DNA damage. In particular, RNA editing enzymes target SSDNA and activate DNA repair pathways that cause the formation of double strand breaks (DSBs). One member of this protein family, activation-induced cytidine deaminase (AID), catalyses the conversion of deoxy-cytidine to deoxy-uracil which is removed by the base-excision repair (BER) enzyme uracil DNA glycosylase creating a DNA lesion in the form of an abasic site (Santos-Pereira and Aguilera, 2015; Sollier and Cimprich, 2015). Besides BER, nucleotide-excision repair (NER) enzymes xeroderma pigmentosum factors $F$ and G (XPF and XPG) process flaps on either side of the R-loop into DSBs.

As is the case in other physiological processes within a cell including DNA replication, the persistent presence of SSDNA causes stress signalling to be activated and needs to be prevented for maintaining genome integrity. The formation and persistence of R-loops is regulated by a number of factors could be a starting point for understanding how the loss of MDM2 leads to an accumulation of R-loops in p53-deficient cells. 


\section{VI.3.2 Defects in ribonucleoproteins cause pre-mRNA to thread back into the DNA}

duplex

R-loop formation has been associated with the loss of several factors involved in mRNA metabolism (Aguilera and García-Muse, 2012; Hamperl and Cimprich, 2014). As the nascent RNA emerges from the RNA polymerase channel, it is coated by heterogeneous nuclear ribonucleoproteins (hnRNPs) to retain the pre-mRNA in the nucleus until it is fully post-transcriptionally modified and spliced. In addition to preventing RNA misfolding into secondary structures, hnRNPs also prevent re-annealing of the strand to its complementary DNA template. So far, MDM2 is only known to interact with hnRNP K, an important co-activator in p53-dependent transcription, and MDM2 can cause its proteasomal degradation (Enge et al., 2009; Moumen et al., 2005). In the context of our data, this role of MDM2 does not explain the increase in R-loop formation in the absence of MDM2. A loss of MDM2 would result in an accumulation rather than a loss of hnRNP $K$ due to a lack of negative regulation. However, we cannot exclude that MDM2 can positively affect other hnRNPs, such that a loss of MDM2 concomitantly leads to a loss in binding proteins or recruitment of splicing factors that would allow nascent RNA to reanneal to its template.

\section{VI.3.3 Topoisomerases suppress R-loop formation by relaxing torsional stress}

R-loop formation is additionally suppressed by the activity of DNA topoisomerases that relieve torsional stress which can be generated by transcription and replication (GarcíaMuse and Aguilera, 2016). In transcription, the RNA polymerase moves along DNA strands and unwinds the double helix for access to the template sequence. The resulting positive and negative supercoils ahead and behind the polymerase are relaxed by topoisomerases I and II. A loss of topoisomerase I not only causes the helix behind the RNA polymerase to be underwound and more accessible to for an intruding RNA strand, but it also impairs ASF/SF2-dependent assembly of messenger ribonucleoprotein (mRNP) during transcription (Tuduri et al., 2009). Both types of topoisomerases have additionally been described to counteract torsional stress and sister chromatid entanglement generated by replicative helicases (Bermejo et al., 2007).

Even though no direct interaction between MDM2 and topoisomerases has been described, a loss of MDM2 seems to sensitise cells towards topoisomerase II inhibition, whereas an MDM2 amplification protects cells from DNA damage when treated with inhibitors to topoisomerase II in a RING finger-dependent but p53-independent way 
(Conradt et al., 2013; Senturk et al., 2017). Along the same lines, p53-deficient cells were also found to be more sensitive to topoisomerase II inhibitors, a phenotype that could be rescued by inhibiting transcriptional elongation (Yeo et al., 2016). As p53-deficient cells will also have low levels of MDM2 due to a lack of MDM2 induction by p53, it seems possible the results observed in this study are a not a direct effect of p53-deficiency but rather caused by a loss of MDM2. Until now, it remains unclear from this data whether the synergism between MDM2 and topoisomerase loss is attributed to the canonical and noncanonical roles of topoisomerases in preventing $\mathrm{R}$-loops or whether replication forks are directly affected by torsional stress in the absence of MDM2.

\section{VI.3.4 Ribonucleases and helicases catalyse the removal of R-loops}

Next to the enzymes that prevent R-loop formation in the first place, there are also a number of enzymes that catalyse their removal. RNase $\mathrm{H}$ endonucleases specifically degrade the RNA moiety of hybrid structures and are found in two forms in human cells $\mathrm{RNase} \mathrm{H} 1$ and $\mathrm{H} 2$. RNase $\mathrm{H} 1$ is monomeric and acts to remove long DNA:RNA hybrids, whereas $\mathrm{RNase} \mathrm{H} 2$ is a trimeric complex and removes single mis-incorporated ribonucleotides from DNA (Reijns et al., 2012). In addition to its roles in protecting mitochondrial replication and telomeres from recombination, $\mathrm{RNaseH} 1$ was also found to play a role in nuclear DNA replication and to support genome integrity (Arora et al., 2014; Parajuli et al., 2017; Suzuki et al., 2010). Apart from nucleases-mediated removal of DNA:RNA hybrids, senataxin (SETX), Aquarius (AQR), and DEAH box protein 9 (DHX9) helicases are thought to unwind stable hybrid structures to facilitate their removal (Chakraborty and Grosse, 2011; Santos-Pereira and Aguilera, 2015; Sollier et al., 2014).

Ectopic expression of RNase $\mathrm{H} 1$ is commonly used to suppress R-loop accumulation and subsequent DNA damage in mammalian cells. We showed that the overexpression of wildtype $\mathrm{RNase} \mathrm{H} 1$ restored replication fork progression that was impaired as a result of MDM2 and RNF2 depletion, whereas a catalytically inactive mutant version of RNase $\mathrm{H} 1$ did not. However, we could not identify a direct correlation between endogenous levels of MDM2 and RNase $\mathrm{H} 1$ and suggest that rescued fork progression is rather due to the removal of R-loops that arise from a different mechanism. Interestingly, a recent study suggested that p53-deficient cells are dependent on DHX9 expression for cell cycle progression and survival (Lee and Pelletier, 2017). 
The susceptibility of p53-deficient cells for two different mechanisms by which cells counteract R-loops suggests that topoisomerase II inhibition and DHX9 depletion mediate the persistence rather than formation of R-loops in the absence of a functional p53-MDM2 axis.

\section{VI.3.5 Transcription - replication conflicts}

In addition to the mechanisms described above, cells also try to avoid the persistence of long R-loops by uncoupling the processes of DNA transcription and replication, both temporally as well as spatially. Replication initiation in human cells is subject to complex regulatory mechanisms, given that only a fraction of licensed origins is fired at one time and the set of origins used differs greatly between cell type and during development. With the development of more sensitive sequencing methods, it was discovered that replication initiates mainly from intergenic regions and is often co-oriented with transcription (Petryk et al., 2016). Replisomes move along the template much faster than transcription factories causing them to catch up and collide when present at the same genomic loci. These codirectional collisions between transcription and replication seem to be much less detrimental in terms of replication compared to collisions that occur "head-on". In this conformation, unwinding of the template from both sides creates an additive positive supercoil in the region between the two polymerase complexes promoting the formation of R-loops (Aguilera and García-Muse, 2012; García-Muse and Aguilera, 2016; Lang et al., 2017). Experiments using a human-cell-based plasmid system even suggested that replisomes can resolve co-transcriptional R-loops via its MCM components when it is encountered co-directionally. Converging replication and transcription on the other hand, cause the formation of R-loops and DNA damage signalling by blocking the transcription machinery and confining the nascent RNA close to its template. Additional torsional stress created by both machineries might facilitate the re-annealing of the transcript to DNA (Hamperl et al., 2017).

We found that inhibiting transcriptional elongation by CDK9 inhibition was sufficient to restore impaired replication forks after MDM2 and RNF2 depletion, likely due to fewer conflicts between transcription and replication. On one hand, changes in chromatin compaction mediated by MDM2/RNF depletion could lead to unscheduled transcription of target genes. On the other hand, the increase in R-loops observed after MDM2 depletion could additionally activate gene expression by preventing promotor methylation. In both cases, increased transcription raises the chances of head-on collisions with DNA 
replication forks. CDK9 inhibition blocks the elongation step of transcription so that RNA polymerases remain associated with the template in an inactive state. This prevents the formation of co-transcriptional R-loops in the time of inhibition but whether it also facilitates the replication fork moving past it, is unclear. This makes it difficult to distinguish whether fork stalling observed in our assays is a direct consequence of transcriptionreplication collisions, replication forks running into R-loops, or even both.

\section{VI.4 Targeting the p53-MDM2 axis in chemotherapy}

The tumour suppressor p53 is mutated in about $50 \%$ of all cancers, ranging from about $10 \%$ to $96 \%$ depending on the cancer entity. Next to mutations, p53 levels in tumours can also be affected by elevated levels of its negative regulators MDM2/MDMX. Genes for both MDM2 and MDMX are amplified in a number of cancers including sarcomas and breast tumours. Given its canonical role of regulating p53 levels, these amplifications make MDM2 and MDMX potent oncogenes and present suitable therapeutic targets to restore p53 levels in cancers (Burgess et al., 2016). The first small-molecule inhibitor targeting MDM2 developed by Vassilev at al. in 2004 and was designed to bind to the $\mathrm{N}$ terminal hydrophobic cleft of MDM2, a binding site for p53 (Vassilev et al., 2004). Unfortunately, this inhibitor (Nutlin-3a) as well as many similar derivatives were promising during pre-clinical development but failed clinical trials due to poor bioavailability and high cytotoxicity (Ray-Coquard et al., 2012).

Our results highlighted an important role of both p53 and MDM2 in supporting DNA replication and genome integrity. An inhibitor targeting the N-terminal p53-binding site on MDM2 (Nutlin-3a) was able to accumulate p53 and reduce replication fork stalling in cells, adding to the guardian of the genome role of p53. As we hypothesised that MDM2 acts downstream and independently of p53 to support DNA replication via its RING domain, it would be of interest to study the effects of inhibitors targeting the RING domain of MDM2. Inhibitors that could be tested include HLI373, thought to inhibit E3 ligase activity of MDM2, as well as MX69, described to lead to MDM2 degradation. For comparison, RG7388 an inhibitor targeting the p53-binding domain of MDM2 could also be tested in several cancer cell lines (Ding et al., 2013; Gu et al., 2016; Kitagaki et al., 2008).

Our results highlighted that a loss of MDM2 has two different effects on DNA replication in p53-proficient cells. On one hand, the inability to bind to p53 results in a loss of negative regulation and accumulation of p53 which is accompanied by more processive DNA replication. Our work in p53-null cells has also highlighted that its RING domain with 
intrinsic E3 ubiquitin ligase activity is required to support DNA replication. As MDM2 is known to be induced by active p53 transcription factor, an accumulation of p53 would also result in increased MDM2 protein levels and enhanced DNA replication. As a result of this complicated feedback loop, it seems essential to distinguish between a complete loss of MDM2, an inhibition of its p53-binding ability, and an inhibition of its C-terminal RING domain. This consideration should also be taken into account when targeting the p53MDM2 axis with small-molecule inhibitors. Treatment of tumours containing wildtype p53 with inhibitors like Nutlin-3a or Isadanutlin (RG7388) targeting the interaction of MDM2 and p53 causes an accumulation of both proteins and supports DNA replication in the cells rather than causing the anticipated genomic instability. In this context, it would be more suitable to target both, p53-binding and RING domains of MDM2 in order to induce replicative stress via the RING domain inhibition and apoptosis pathways via p53 accumulation. For this, inhibitors targeting the RING activity of MDM2 need to be further developed and brought forward to clinical trials.

\section{VI.5 Conclusions}

In conclusion, our work has highlighted an important non-canonical pathway of the p53MDM2 network in tumour suppression. It adds to the role of p53 as the guardian of the genome by supporting DNA replication and suppressing genomic instability via its target gene MDM2. It supports DNA replication by preventing the formation and persistence of $\mathrm{R}$-loops that pose as obstacles to replisomes and cause them to stall. Resulting underreplicated DNA is a frequent cause for genomic instability found in cancers. The exact mechanism of how MDM2 depletion leads to R-loop formation remains subject to further studies. For this, understanding exactly how MDM2 mediates chromatin marks and how its depletion affects transcription could provide vital information on the mechanism. Targeting the p53-MDM2 network remains an attractive strategy in chemotherapy and should be further explored in terms of inhibitors targeting the RING domain of MDM2 that is responsible for DNA replication support and combination therapies of inhibitors targeting different domains of MDM2 in tumours bearing wildtype p53. 


\section{References}

Adriaens, C., Standaert, L., Barra, J., Latil, M., Verfaillie, A., Kalev, P., Boeckx, B., Wijnhoven, P.W.G., Radaelli, E., Vermi, W., et al. (2016). P53 induces formation of NEAT1 IncRNA-containing paraspeckles that modulate replication stress response and chemosensitivity. Nat. Med. 22, 861868.

Aguilera, A., and García-Muse, T. (2012). R Loops: From Transcription Byproducts to Threats to Genome Stability. Mol. Cell 46, 115-124.

Alabert, C., and Groth, A. (2012). Chromatin replication and epigenome maintenance. Nat. Rev. Mol. Cell Biol. 13, 153-167.

Alberts, B., Johnsen, A., Lewis, J., Raff, M., Roberts, K., and Walker, P. (2007). Molecular Biology of the Cell.

Allen, M.A., Andrysik, Z., Dengler, V.L., Mellert, H.S., Guarnieri, A., Freeman, J.A., Sullivan, K.D., Galbraith, M.D., Luo, X., Kraus, W.L., et al. (2014). Global analysis of p53-regulated transcription identifies its direct targets and unexpected regulatory mechanisms. Elife 3, e02200.

Alt, J.R., Bouska, A., Fernandez, M.R., Cerny, R.L., Xiao, H., and Eischen, C.M. (2005). Mdm2 binds to Nbs1 at sites of DNA damage and regulates double strand break repair. J. Biol. Chem. 280, 18771-18781.

Argentini, M., Barboule, N., and Wasylyk, B. (2001). The contribution of the acidic domain of MDM2 to p53 and MDM2 stability. Oncogene 20, 1267-1275.

Arias-Lopez, C., Lazaro-Trueba, I., Kerr, P., Lord, C.J., Dexter, T., Iravani, M., Ashworth, A., and Silva, A. (2006). p53 modulates homologous recombination by transcriptional regulation of the RAD51 gene. EMBO Rep. 7, 219-224.

Arora, R., Lee, Y., Wischnewski, H., Brun, C.M., Schwarz, T., and Azzalin, C.M. (2014). RNaseH1 regulates TERRA-telomeric DNA hybrids and telomere maintenance in ALT tumour cells. Nat. Commun. 5, 1-11.

Baptiste, N., Friedlander, P., Chen, X., and Prives, C. (2002). The proline-rich domain of p53 is required for cooperation with anti-neoplastic agents to promote apoptosis of tumor cells. Oncogene 21, 9-21.

Barak, Y., Gottlieb, E., Juven-Gershon, T., and Oren, M. (1994). Regulation of mdm2 expression by p53: Alternative promoters produce transcripts with nonidentical translation potential. Genes Dev. 8, 1739-1749.

Barker, P.E. (1982). Double minutes in human tumor cells. Cancer Genet. Cytogenet. 5, 81-94.

Bartel, F., Schulz, J., Böhnke, A., Blümke, K., Kappler, M., Bache, M., Schmidt, H., Würl, P., Taubert, H., and Hauptmann, S. (2005). Significance of HDMX-S ( or MDM4 ) mRNA splice variant overexpression and HDMX gene amplification on primary soft tissue sarcoma prognosis. Int. J. Cancer 469-475.

Bartkova, J., Horejsí, Z., Koed, K., Krämer, A., Tort, F., Zieger, K., Guldberg, P., Sehested, M., Nesland, J.M., Lukas, C., et al. (2005). DNA damage response as a candidate anti-cancer barrier in early human tumorigenesis. Nature $434,864-870$.

Bartkova, J., Rezaei, N., Liontos, M., Karakaidos, P., Kletsas, D., Issaeva, N., Vassiliou, L.-V.F., Kolettas, E., Niforou, K., Zoumpourlis, V.C., et al. (2006). Oncogene-induced senescence is part of the tumorigenesis barrier imposed by DNA damage checkpoints. Nature 444, 633-637. 
Bartz, S.R., Zhang, Z., Burchard, J., Imakura, M., Martin, M., Palmieri, A., Needham, R., Guo, J., Gordon, M., Chung, N., et al. (2006). Small Interfering RNA Screens Reveal Enhanced Cisplatin Cytotoxicity in Tumor Cells Having both BRCA Network and TP53 Disruptions. Mol. Cell. Biol. 26, 9377-9386.

Baugh, E.H., Ke, H., Levine, A.J., Bonneau, R.A., and Chan, C.S. (2017). Why are there hotspot mutations in the TP53 gene in human cancers? Cell Death Differ. 25, 154-160.

Becker, J.S., Nicetto, D., and Zaret, K.S. (2016). H3K9me3-Dependent Heterochromatin: Barrier to Cell Fate Changes. Trends Genet. 32, 29-41.

Beli, P., Lukashchuk, N., Wagner, S.A., Weinert, B.T., Olsen, J. V., Baskcomb, L., Mann, M., Jackson, S.P., and Choudhary, C. (2012). Proteomic Investigations Reveal a Role for RNA Processing Factor THRAP3 in the DNA Damage Response. Mol. Cell 46, 212-225.

Bermejo, R., Doksani, Y., Capra, T., Katou, Y.M., Tanaka, H., Shirahige, K., and Foiani, M. (2007). Top1- and Top2-mediated topological transitions at replication forks ensure fork progression and stability and prevent DNA damage checkpoint activation. Genes Dev. 21, 1921-1936.

Bermejo, R., Lai, M.S., and Foiani, M. (2012). Preventing Replication Stress to Maintain Genome Stability: Resolving Conflicts between Replication and Transcription. Mol. Cell 45, 710-718.

Bertrand, P., Saintigny, Y., and Lopez, B.S. (2004). p53's double life: Transactivation-independent repression of homologous recombination. Trends Genet. 20, 235-243.

Bieging, K.T., Mello, S.S., and Attardi, L.D. (2014). Unravelling mechanisms of p53-mediated tumour suppression. Nat. Rev. Cancer 14, 359-370.

Bittner, M., Kupferer, P., and Morris, C.F. (1980). Electrophoretic transfer of proteins and nucleic acids from slab gels to diazobenzyloxymethyl cellulose or nitrocellulose sheets. Anal. Biochem. 102, 459-471.

Blackledge, N.P., Rose, N.R., and Klose, R.J. (2015). Targeting Polycomb systems to regulate gene expression: Modifications to a complex story. Nat. Rev. Mol. Cell Biol. 16, 643-649.

De Bont, R. (2004). Endogenous DNA damage in humans: a review of quantitative data. Mutagenesis 19, 169-185.

Bootsma, D., Budke, L., and Vos, O. (1964). Studies on synchronous division of tissue culture cells initiated by excess thymidine. Exp. Cell Res. 33, 301-309.

Borden, K.L., and Freemont, P.S. (1996). The RING finger domain: a recent example of a sequence-structure family. Curr. Opin. Struct. Biol. 6, 395-401.

Bouska, A., and Eischen, C.M. (2009). Murine double minute 2: p53-independent roads lead to genome instability or death. Trends Biochem. Sci. 34, 279-286.

Bouska, A., Lushnikova, T., Plaza, S., and Eischen, C.M. (2008). Mdm2 promotes genetic instability and transformation independent of p53. Mol. Cell. Biol. 28, 4862-4874.

Boyer, L. a, Plath, K., Zeitlinger, J., Brambrink, T., Medeiros, L. a, Lee, T.I., Levine, S.S., Wernig, M., Tajonar, A., Ray, M.K., et al. (2006). Polycomb complexes repress developmental regulators in murine embryonic stem cells. Nature 441, 349-353.

Brady, C.A., Jiang, D., Mello, S.S., Johnson, T.M., Jarvis, L.A., Kozak, M.M., Broz, D.K., Basak, S., Park, E.J., McLaughlin, M.E., et al. (2011). Distinct p53 transcriptional programs dictate acute DNA-damage responses and tumor suppression. Cell 145, 571-583. 
Branzei, D., and Foiani, M. (2005). The DNA damage response during DNA replication. Curr. Opin. Cell Biol. 17, 568-575.

Brown, D.R., Thomas, C.A., and Deb, S.P. (1998). The human oncoprotein MDM2 arrests the cell cycle: Elimination of its cell-cycle-inhibitory function induces tumorigenesis. EMBO J. 17, 25132525.

Bruhn, C., Zhou, Z.W., Ai, H., and Wang, Z.Q. (2014). The essential function of the MRN complex in the resolution of endogenous replication intermediates. Cell Rep. 6, 182-195.

Bunz, F., Dutriaux, a, Lengauer, C., Waldman, T., Zhou, S., Brown, J.P., Sedivy, J.M., Kinzler, K.W., and Vogelstein, B. (1998). Requirement for p53 and p21 to sustain G2 arrest after DNA damage. Science 282, 1497-1501.

Bunz, F., Hwang, P.M., Torrance, C., Waldman, T., Zhang, Y., Dillehay, L., Williams, J., Lengauer, C., Kinzler, K.W., and Vogelstein, B. (1999). Disruption of p53 in human cancer cells alters the responses to therapeutic agents. J Clin Invest 104, 263-269.

Burgess, A., Chia, K.M., Haupt, S., Thomas, D., Haupt, Y., and Lim, E. (2016). Clinical Overview of MDM2/X-Targeted Therapies. Front. Oncol. 6, 1-7.

Byun, T.S., Pacek, M., Yee, M., Walter, J.C., and Cimprich, K.A. (2005). Functional uncoupling of MCM helicase and DNA polymerase activities activates the ATR-dependent checkpoint. 10401052.

Cahilly-Snyder, L., Yang-Feng, T., Francke, U., and George, D. (1987). Molecular analysis and chromosomal mapping of amplified genes isolated from a transformed mouse 3T3 cell line. Somat Cell Mol Genet 3, 235-244.

Candau, R., Scolnick, D.M., Darpino, P., Ying, C.Y., Halazonetis, T.D., and Berger, S.L. (1997). Two tandem and independent sub-activation domains in the amino terminus of p53 require the adaptor complex for activity. Oncogene 15, 807-816.

Carr, M.I., Roderick, J.E., Gannon, H.S., Kelliher, M.A., and Jones, S.N. (2016). Mdm2 Phosphorylation Regulates Its Stability and Has Contrasting Effects on Oncogene and RadiationInduced Tumorigenesis. Cell Rep. 16, 2618-2629.

Carrillo, A.M., Bouska, A., Arrate, M.P., and Eischen, C.M. (2014). Mdmx promotes genomic instability independent of p53 and Mdm2. Oncogene 34, 1-11.

Cayrou, C., Coulombe, P., Vigneron, A., Stanojcic, S., Ganier, O., Peiffer, I., Rivals, E., Puy, A., Laurent-Chabalier, S., Desprat, R., et al. (2011). Genome-scale analysis of metazoan replication origins reveals their organization in specific but flexible sites defined by conserved features. Genome Res. 21, 1438-1449.

Cayrou, C., Coulombe, P., Puy, A., Rialle, S., Kaplan, N., Segal, E., and Méchali, M. (2012). New insights into replication origin characteristics in metazoans. Cell Cycle 11,658-667.

Chakraborty, P., and Grosse, F. (2011). Human DHX9 helicase preferentially unwinds RNAcontaining displacement loops (R-loops) and G-quadruplexes. DNA Repair (Amst). 10, 654-665.

Chang, J., Kim, D.H., Seung Woo Lee, Kwan Yong Choi, and Young Chul Sung (1995). Transactivation ability of p53 transcriptional activation domain is directly related to the binding affinity to TATA-binding protein. J. Biol. Chem. 270, 25014-25019.

Chaudhuri, A.R., Callen, E., Ding, X., Gogola, E., Duarte, A.A., Lee, J.E., Wong, N., Lafarga, V., Calvo, J.A., Panzarino, N.J., et al. (2016). Replication fork stability confers chemoresistance in BRCA-deficient cells. Nature 535, 382-387. 
Chen, L., Li, Z., Zwolinska, A.K., Smith, M. a, Cross, B., Koomen, J., Yuan, Z.-M., Jenuwein, T., Marine, J.-C., Wright, K.L., et al. (2010). MDM2 recruitment of lysine methyltransferases regulates p53 transcriptional output. EMBO J. 29, 2538-2552.

Chène, P. (2001). The role of tetramerization in p53 function. Oncogene 20, 2611-2617.

Conradt, L., Henrich, A., Wirth, M., Reichert, M., Lesina, M., Algül, H., Schmid, R.M., Krämer, O.H., Saur, D., and Schneider, G. (2013). Mdm2 inhibitors synergize with topoisomerase II inhibitors to induce p53-independent pancreatic cancer cell death. Int. J. Cancer 132, 2248-2257.

Conti, C., Saccà, B., Herrick, J., Lalou, C., Pommier, Y., and Bensimon, A. (2007). Replication fork velocities at adjacent replication origins are coordinately modified during DNA replication in human cells. Mol. Biol. Cell 18, 3059-3067.

Cordon-Cardo, C., Latres, E., Drobnjak, M., Oliva, M.R., Pollack, D., Woodruff, J.M., Marechal, V., Chen, J., Brennan, M.F., and Levine, A.J. (1994). Molecular Abnormalities of mdm2 and p53 Genes in Adult Soft Tissue Sarcomas. Cancer Res. 54, 794-799.

Cristini, A., Groh, M., Kristiansen, M.S., and Gromak, N. (2018). RNA/DNA Hybrid Interactome Identifies DXH9 as a Molecular Player in Transcriptional Termination and R-Loop-Associated DNA Damage. Cell Rep. 23, 1891-1905.

Cross, B., Chen, L., Cheng, Q., Li, B., Yuan, Z.-M., and Chen, J. (2011). Inhibition of p53 DNA binding function by the MDM2 protein acidic domain. J. Biol. Chem. 286, 16018-16029.

Deans, A.J., and West, S.C. (2011). DNA interstrand crosslink repair and cancer. Nat. Rev. Cancer $11,467-480$.

Ding, Q., Zhang, Z., Liu, J.-J., Jiang, N., Zhang, J., Ross, T.M., Chu, X.-J., Bartkovitz, D., Podlaski, F., Janson, C., et al. (2013). Discovery of RG7388, a Potent and Selective p53-MDM2 Inhibitor in Clinical Development. J. Med. Chem. 56, 5979-5983.

Dittmer, D., Pati, S., Zambetti, G., Chu, S., Teresky, A.K., Moore, M., Finlay, C., and Levine, A.J. (1993). Gain of function mutations in p53. Nat. Genet. 4.

Dobbelstein, M., and Moll, U. (2014). Targeting tumour-supportive cellular machineries in anticancer drug development. Nat. Rev. Drug Discov. 13, 179.

Dobbelstein, M., and Sørensen, C.S. (2015). Exploiting replicative stress to treat cancer. Nat. Rev. Drug Discov. 14, 405-423.

Donehower, L.A., Harvey, M., Slagle, B.L., McArthur, M.J., Montgomery, C.A., Butel, J.S., and Bradley, A. (1992). Mice deficient for p53 are developmentally normal but susceptible to spontaneous tumours. Nature 356, 215-221.

Dudgeon, C., Chan, C., Kang, W., Sun, Y., Emerson, R., Robins, H., and Levine, A.J. (2014). The evolution of thymic lymphomas in p53 knockout mice. Genes Dev. 28, 2613-2620.

Duursma, A.M., Driscoll, R., Elias, J.E., and Cimprich, K.A. (2013). A Role for the MRN Complex in ATR Activation via TOPBP1 Recruitment. Mol. Cell 50,116-122.

Eischen, C.M. (2017). Role of Mdm 2 and Mdmx in DNA repair. 9, 1-5.

Enge, M., Bao, W., Hedström, E., Jackson, S.P., Moumen, A., and Selivanova, G. (2009). MDM2Dependent Downregulation of p21 and hnRNP K Provides a Switch between Apoptosis and Growth Arrest Induced by Pharmacologically Activated p53. Cancer Cell 15, 171-183.

Ewald, B., Sampath, D., and Plunkett, W. (2008). Nucleoside analogs: Molecular mechanisms signaling cell death. Oncogene 27, 6522-6537. 
Fåhraeus, R., and Olivares-Illana, V. (2014). MDM2's social network. Oncogene 33, 4365-4376.

Fakharzadeh, S.S., Trusko, S.P., and George, D.L. (1991). Tumorigenic potential associated with enhanced expression of a gene that is amplified in a mouse tumor cell line. EMBO J. 10, 15651569.

Falck, J., Petrini, J.H.J., Williams, B.R., Lukas, J., and Bartek, J. (2002). The DNA damagedependent intra-S phase checkpoint is regulated by parallel pathways. Nat. Genet. 30, 290-294.

Fang, S., Jensen, J.P., Ludwig, R.L., Vousden, K.H., and Weissman, A.M. (2000). Mdm2 is a RING finger-dependent ubiquitin protein ligase for itself and p53. J. Biol. Chem. 275, 8945-8951.

Faust, C., Schumacher, a, Holdener, B., and Magnuson, T. (1995). The eed mutation disrupts anterior mesoderm production in mice. Development 121, 273-285.

Fearon, E.F., and Vogelstein, B. (1990). A genetic model for colorectal tumorigenesis. Cell 61, 759-767.

Fischle, W., Wang, Y., Jacobs, S.A., Kim, Y., Allis, C.D., and Khorasanizadeh, S. (2003). Molecular basis for the discrimination of repressive methyl-lysine marks in histone H3 by polycomb and HP1 chromodomains. Genes Dev. 17, 1870-1881.

Friedler, A., Veprintsev, D.B., Freund, S.M.V., Von Glos, K.I., and Fersht, A.R. (2005). Modulation of binding of DNA to the C-terminal domain of p53 by acetylation. Structure $13,629-636$.

Frum, R. a, Singh, S., Vaughan, C., Mukhopadhyay, N.D., Grossman, S.R., Windle, B., Deb, S., and Deb, S.P. (2014). The human oncoprotein MDM2 induces replication stress eliciting early intraS-phase checkpoint response and inhibition of DNA replication origin firing. Nucleic Acids Res. 42, 926-940.

Fu, D., Calvo, J.A., and Samson, L.D. (2012). Balancing repair and tolerance of DNA damage caused by alkylating agents. Nat. Rev. Cancer 12, 104-120.

Gajjar, M., Candeias, M.M., Malbert-Colas, L., Mazars, A., Fujita, J., Olivares-Illana, V., and Fåhraeus, R. (2012). The p53 mRNA-Mdm2 interaction controls mdm2 nuclear trafficking and is required for p53 activation following dna damage. Cancer Cell 21, 25-35.

Galanos, P., Vougas, K., Walter, D., Polyzos, A., Maya-Mendoza, A., Haagensen, E.J., Kokkalis, A., Roumelioti, F.-M., Gagos, S., Tzetis, M., et al. (2016). Chronic p53-independent p21 expression causes genomic instability by deregulating replication licensing. Nat. Cell Biol. 18.

García-Muse, T., and Aguilera, A. (2016). Transcription-replication conflicts: how they occur and how they are resolved. Nat. Rev. Mol. Cell Biol. 17, 553-563.

Gazdar, A.F., Girard, L., Lockwood, W.W., Lam, W.L., and Minna, J.D. (2010). Lung cancer cell lines as tools for biomedical discovery and research. J. Natl. Cancer Inst. 102, 1310-1321.

Ge, X.Q., Jackson, D. a, and Blow, J.J. (2007). Dormant origins licensed by excess Mcm2-7 are required for human cells to survive replicative stress. Genes Dev. 21, 3331-3341.

Ginno, P.A., Lott, P.L., Christensen, H.C., Korf, I., and Chédin, F. (2012). R-Loop Formation Is a Distinctive Characteristic of Unmethylated Human CpG Island Promoters. Mol. Cell 45, 814-825.

Glover, T.W., Arlt, M.F., Casper, A.M., and Durkin, S.G. (2005). Mechanisms of common fragile site instability. Hum. Mol. Genet. 14, 197-205.

Gorgoulis, V., Vassiliou, L., Karakaidos, P., Zacharatos, P., Kotsinas, A., Lioglou, T., Venere, M., DiTullio, R., Kastrinakis, N., Levy, B., et al. (2005). Activation of the DNA damage checkpoint and genomic instability in human precancerous lesions. Nature 434, 907-913. 
Gorthi, A., Romero, J.C., Loranc, E., Cao, L., Lawrence, L.A., Goodale, E., Iniguez, A.B., Bernard, X., Masamsetti, V.P., Roston, S., et al. (2018). EWS-FLI1 increases transcription to cause R-loops and block BRCA1 repair in Ewing sarcoma. Nature.

Gottifredi, V., Shieh, S., Taya, Y., and Prives, C. (2001). p53 accumulates but is functionally impaired when DNA synthesis is blocked. Proc. Natl. Acad. Sci. U. S. A. 98, 1036-1041.

Gu, L., Zhu, N., Zhang, H., Durden, D.L., Feng, Y., and Zhou, M. (2009). Regulation of XIAP translation and induction by MDM2 following irradiation. Cancer Cell 15, 363-375.

Gu, L., Zhang, H., Liu, T., Zhou, S., Du, Y., Xiong, J., Yi, S., Qu, C.K., Fu, H., and Zhou, M. (2016). Discovery of Dual Inhibitors of MDM2 and XIAP for Cancer Treatment. Cancer Cell 30, 623-636.

Halazonetis, T.D., Gorgoulis, V.G., and Bartek, J. (2008). An oncogene-induced DNA damage model for cancer development. Science 319, 1352-1355.

Hamperl, S., and Cimprich, K.A. (2014). The contribution of co-transcriptional RNA: DNA hybrid structures to DNA damage and genome instability. DNA Repair (Amst). 19, 84-94.

Hamperl, S., Bocek, M.J., Saldivar, J.C., Swigut, T., and Cimprich, K.A. (2017). TranscriptionReplication Conflict Orientation Modulates R-Loop Levels and Activates Distinct DNA Damage Responses. Cell 170, 774-786.e19.

Hampp, S., Kiessling, T., Buechle, K., Mansilla, S.F., Thomale, J., and Rall, M. (2016). DNA damage tolerance pathway involving DNA polymerase I and the tumor suppressor p53 regulates DNA replication fork progression.

Hanahan, D., and Weinberg, R. a. (2011). Hallmarks of cancer: The next generation. Cell 144, 646-674.

Harper, J.W., Elledge, S.J., Keyomarsi, K., Dynlacht, B., Tsai, L.H., Zhang, P., Dobrowolski, S., Bai, C., Connell-Crowley, L., and Swindell, E. (1995). Inhibition of cyclin-dependent kinases by p21. Mol. Biol. Cell 6, 387-400.

Haupt, Y., Maya, R., Kazaz, A., and Oren, M. (1997). Mdm2 promotes the rapid degradation of p53. Nature 387, 296-299.

Henry-Mowatt, J., Jackson, D., Masson, J.Y., Johnson, P.A., Clements, P.M., Benson, F.E., Thompson, L.H., Takeda, S., West, S.C., and Caldecott, K.W. (2003). XRCC3 and Rad51 modulate replication fork progression on damaged vertebrate chromosomes. Mol. Cell 11, 11091117.

Hermeking, H., Lengauer, C., Polyak, K., He, T., Zhang, L., Thiagalingam, S., Kinzler, K.W., and Vogelstein, B. (1997). 14-3-3r Is a p53-Regulated Inhibitor of G2 / M Progression. Mol. Cell 1, 311.

Hernández-Monge, J., Rousset-Roman, A.B., Medina-Medina, I., and Olivares-Illana, V. (2016). Dual function of MDM2 and MDMX toward the tumor suppressors p53 and RB. Genes Cancer 7, 278-287.

Honda, R., Tanaka, H., and Yasuda, H. (1997). Oncoprotein MDM2 is a ubiquitin ligase E3 for tumor suppressor p53. FEBS Lett. 420, 25-27.

Horn, H.F., and Vousden, K.H. (2007). Coping with stress: multiple ways to activate p53. Oncogene 26, 1306-1316.

Huertas, P., and Aguilera, A. (2003). Cotranscriptionally formed DNA:RNA hybrids mediate transcription elongation impairment and transcription-associated recombination. Mol. Cell 12, $711-$ 721. 
Itahana, K., Mao, H., Jin, A., Itahana, Y., Clegg, H. V., Lindström, M.S., Bhat, K.P.P., Godfrey, V.L., Evan, G.I., and Zhang, Y. (2007). Targeted Inactivation of Mdm2 RING Finger E3 Ubiquitin Ligase Activity in the Mouse Reveals Mechanistic Insights into p53 Regulation. Cancer Cell 12, 355-366.

Iwakuma, T., and Lozano, G. (2003). MDM2 , An Introduction. 1, 993-1000.

Jackson, D., and Pombo, A. (1998). Replicon clusters are stable units of chromosome structure: evidence that nuclear organization contributes to the efficient activation and propagation of $S$ phase in human cells. J. Cell Biol. 140, 1285-1295.

Jain, A.K., and Barton, M.C. (2016). Outside the p53 RING: Transcription Regulation by ChromatinBound MDM2. Mol. Cell 62, 805-807.

Janz, C., and Wiesmüller, L. (2002). Wild-type p53 inhibits replication-associated homologous recombination. Oncogene 21, 5929-5933.

Jenkins, L.M.M., Durell, S.R., Mazur, S.J., and Appella, E. (2012). p53 N-terminal phosphorylation: A defining layer of complex regulation. Carcinogenesis 33, 1441-1449.

Jones, S.N., Hancock, A.R., Vogel, H., Donehower, L.A., and Bradley, A. (1998). Overexpression of Mdm2 in mice reveals a p53-independent role for Mdm2 in tumorigenesis. Proc. Natl. Acad. Sci. U.S. A. $95,15608-15612$.

Jonkers, J., Meuwissen, R., van der Gulden, H., Peterse, H., van der Valk, M., and Berns, A. (2001). Synergistic tumor suppressor activity of BRCA2 and p53 in a conditional mouse model for breast cancer. Nat. Genet. 29, 418-425.

Junttila, M.R., Karnezis, A.N., Garcia, D., Madriles, F., Kortlever, R.M., Rostker, F., Brown Swigart, L., Pham, D.M., Seo, Y., Evan, G.I., et al. (2010). Selective activation of p53-mediated tumour suppression in high-grade tumours. Nature 468, 567-571.

Jürgens, G. (1985). A group of genes controlling the spatial expression of the bithorax complex in Drosophila. Nature 316, 153-155.

Kawamura, T., Suzuki, J., and Wang, Y. (2009). Linking the p53 tumor suppressor pathway to somatic cell reprogramming. Nature 460, 1140-1144.

Kearns, S., Lurz, R., Orlova, E. V., and Okorokov, A.L. (2016). Two p53 tetramers bind one consensus DNA response element. Nucleic Acids Res. 44, 6185-6199.

Kenzelmann Broz, D., Mello, S.S., Bieging, K.T., Jiang, D., Dusek, R.L., Brady, C.A., Sidow, A., and Attardi, L.D. (2013). Global genomic profiling reveals an extensive p53-regulated autophagy program contributing to key p53 responses. Genes Dev. 27, 1016-1031.

Kim, N., and Jinks-Robertson, S. (2011). Guanine repeat-containing sequences confer transcription-dependent instability in an orientation-specific manner in yeast. DNA Repair (Amst). $10,953-960$.

Kitagaki, J., Agama, K.K., Pommier, Y., Yang, Y., and Weissman, A.M. (2008). Targeting tumor cells expressing p53 with a water-soluble inhibitor of Hdm2. Mol. Cancer Ther. 7, 2445-2454.

Klusmann, I., Rodewald, S., Mueller, L., Friedrich, M., Wienken, M., Li, Y., Schulz-Heddergott, R. and Dobbelstein, M. (2016). p53 Activity Results in DNA Replication Fork Processivity. Cell Rep. 17, 1845-1857.

Kobet, E., Zeng, X., Zhu, Y., Keller, D., and Lu, H. (2000). MDM2 inhibits p300-mediated p53 acetylation and activation by forming a ternary complex with the two proteins. Proc. Natl. Acad. Sci. U. S. A. $97,12547-12552$. 
Kranz, D., and Dobbelstein, M. (2006). Nongenotoxic p53 activation protects cells against S-phasespecific chemotherapy. Cancer Res. 66, 10274-10280.

Kranz, D., Dohmesen, C., and Dobbelstein, M. (2008). BRCA1 and Tip60 determine the cellular response to ultraviolet irradiation through distinct pathways. J. Cell Biol. 182, 197-213.

Krastev, D.B., Slabicki, M., Paszkowski-Rogacz, M., Hubner, N.C., Junqueira, M., Shevchenko, A., Mann, M., Neugebauer, K.M., and Buchholz, F. (2011). A systematic RNAi synthetic interaction screen reveals a link between p53 and snoRNP assembly. Nat. Cell Biol. 13, 809-818.

Krizhanovsky, V., and Lowe, S.W. (2009). Stem cells: The promises and perils of p53. Nature 460, 1085-1086.

Kruiswijk, F., Labuschagne, C.F., and Vousden, K.H. (2015). P53 in survival, death and metabolic health: A lifeguard with a licence to kill. Nat. Rev. Mol. Cell Biol. 16, 393-405.

Kussie, P.H., Gorina, S., Marechal, V., Elenbaas, B., Moreau, J., Levine, a J., and Pavletich, N.P. (1996). Structure of the MDM2 oncoprotein bound to the p53 tumor suppressor transactivation domain. Science 274, 948-953.

Lane, D.P. (1992). Cancer. p53, guardian of the genome. Nature 358, 15-16.

Lane, D.P., and Crawford, L. V (1979). T antigen is bound to a host protein in SY40-transformed cells [19]. Nature 278, 261-263.

Lang, K.S., Hall, A.N., Merrikh, C.N., Ragheb, M., Tabakh, H., Pollock, A.J., Woodward, J.J., Dreifus, J.E., and Merrikh, H. (2017). Replication-Transcription Conflicts Generate R-Loops that Orchestrate Bacterial Stress Survival and Pathogenesis. Cell 170, 787-799.e18.

Lavin, M.F. (2008). Ataxia-telangiectasia: From a rare disorder to a paradigm for cell signalling and cancer. Nat. Rev. Mol. Cell Biol. 9, 759-769.

Lavin, M.F., and Gueven, N. (2006). The complexity of p53 stabilization and activation. Cell Death Differ. 13, 941-950.

Lebofsky, R., and Walter, J.C. (2007). New Myc-anisms for DNA Replication and Tumorigenesis? Cancer Cell 12, 102-103.

Lee, J., and Paull, T.T. (2005). ATM Activation by DNA Double-Strand Breaks Through the Mre11Rad50-Nbs1 Complex. Science (80-. ). 308, 551-554.

Lee, T., and Pelletier, J. (2017). Dependence of p53-deficient cells on the DHX9 DExH-box helicase. Oncotarget 8, 30908-30921.

Lee, S., Elenbaas, B., Levine, a, and Griffith, J. (1995). p53 and its 14 kDa C-terminal domain recognize primary DNA damage in the form of insertion/deletion mismatches. Cell 81, 1013-1020.

Lee, W., Harvey, T.S., Yin, Y., Yau, P., Litchfield, D., and Arrowsmith, C.H. (1994). Solution structure of the tetrameric minimum transforming domain of p53. Nature 1, 877-890.

Lehmann, A.R., Niimi, A., Ogi, T., Brown, S., Sabbioneda, S., Wing, J.F., Kannouche, P.L., and Green, C.M. (2007). Translesion synthesis: Y-family polymerases and the polymerase switch. DNA Repair (Amst). 6, 891-899.

Lemon, B., and Tjian, R. (2000). Orchestrated response: A symphony of transcription factors for gene control. Genes Dev. 14, 2551-2569.

Lenos, K., Grawenda, A.M., Lodder, K., Kuijjer, M.L., Teunisse, A.F.A.S., Repapi, E., Grochola, L.F., Bartel, F., Hogendoorn, P.C.W., Wuerl, P., et al. (2012). Alternate Splicing of the p53 Inhibitor 
HDMX Offers a Superior Prognostic Biomarker than p53 Mutation in Human Cancer. 72, 40744085.

Lerner, L.K., Francisco, G., Soltys, D.T., Rocha, C.R.R., Quinet, A., Vessoni, A.T., Castro, L.P., David, T.I.P., Bustos, S.O., Strauss, B.E., et al. (2017). Predominant role of DNA polymerase eta and p53-dependent translesion synthesis in the survival of ultraviolet-irradiated human cells. Nucleic Acids Res. 45, 1-15.

Leung, K.H.T., El Hassan, M.A., and Bremner, R. (2013). A rapid and efficient method to purify proteins at replication forks under native conditions. Biotechniques 55, 204-206.

Levine, S.S., Weiss, A., Erdjument-Bromage, H., Shao, Z., Tempst, P., and Kingston, R.E. (2002). The Core of the Polycomb Repressive Complex Is Compositionally and Functionally Conserved in Flies and Humans. Mol. Cell. Biol. 22, 6070-6078.

Lewis, E. (1978). A gene complex controlling segmentation in Drosophila. Nature 276, 565-570.

Lewis, P.H. (1949). Pc: Polycomb. Drosoph. Inf. Serv. 21.

Li, J., and Kurokawa, M. (2015). Regulation of MDM2 Stability After DNA Damage. J. Cell. Physiol. 230, 2318-2327.

Li, Q., and Lozano, G. (2013). Molecular pathways: Targeting Mdm2 and Mdm4 in cancer therapy. Clin. Cancer Res. 19, 34-41.

Li, M., Brooks, C.L., Wu-Baer, F., Chen, D., Baer, R., and Gu, W. (2003). Mono- Versus Polyubiquitination: Differential Control of p53 Fate by Mdm2. Science (80-. ). 302, 1972-1975.

Li, T., Kon, N., Jiang, L., Tan, M., Ludwig, T., Zhao, Y., Baer, R., and Gu, W. (2012). Tumor suppression in the absence of p53-mediated cell-cycle arrest, apoptosis, and senescence. Cell $149,1269-1283$.

Li, Y., Saini, P., Sriraman, A., and Dobbelstein, M. (2015). Mdm2 inhibition confers protection of p53-proficient cells from the cytotoxic effects of Wee1 inhibitors. Oncotarget 6, 32339-32352.

Linzer, D.I.H., and Levine, a. J. (1979). Characterization Tumor Antigen and Uninfected of a 54K Dalton Cellular SV40 Present in SV40-Transformed Cells. Cell 17, 43-52.

Lohrum, M.A.E., Woods, D.B., Ludwig, R.L., and Vousden, K.H. (2001). C-Terminal Ubiquitination of p53 Contributes to Nuclear Export. 21, 8521-8532.

Lu, M., Wikman, F., Orntoft, T.F., Methods, A., and Charytonowicz, E. (2002). Impact of Alterations Affecting the p53 Pathway in Bladder Cancer on Clinical Outcome, Assessed by Conventional and Array-based Methods Impact of Alterations Affecting the p53 Pathway in Bladder Cancer on Clinical Outcome, Assessed by Conventional and. 8, 171-179.

Lushnikova, T., Bouska, A., Odvody, J., Dupont, W.D., and Eischen, C.M. (2011). Aging mice have increased chromosome instability that is exacerbated by elevated Mdm2 expression. Oncogene 30 , 4622-4631.

Mailand, N., Falck, J., Lukas, C., Syljuâsen, R.G., Welcker, M., Bartek, J., and Lukas, J. (2000). Rapid destruction of human Cdc25A in response to DNA damage. Science 288, 1425-1429.

Malbert-Colas, L., Ponnuswamy, A., Olivares-Illana, V., Tournillon, A.S., Naski, N., and Fåhraeus, R. (2014). HDMX Folds the Nascent p53 mRNA following Activation by the ATM Kinase. Mol. Cell $54,500-511$. 
Mansilla, S.F., Soria, G., Vallerga, M.B., Habif, M., Martínez-López, W., Prives, C., and Gottifredi, V. (2013). UV-triggered p21 degradation facilitates damaged-DNA replication and preserves genomic stability. Nucleic Acids Res. 41, 6942-6951.

Mansilla, S.F., Bertolin, A.P., Bergoglio, V., Pillaire, M.J., González Besteiro, M. a., Luzzani, C., Miriuka, S.G., Cazaux, C., Hoffmann, J.S., and Gottifredi, V. (2016). Cyclin Kinase-independent role of p21CDKN1A in the promotion of nascent DNA elongation in unstressed cells. Elife 5, 1-26.

Maréchal, A., and Zou, L. (2013). DNA damage sensing by the ATM and ATR kinases. Cold Spring Harb. Perspect. Biol. 5.

Margueron, R., and Reinberg, D. (2011). The Polycomb complex PRC2 and its mark in life. Nature 469, 343-349.

Margueron, R., Li, G., Sarma, K., Blais, A., Zavadil, J., Woodcock, C.L., Dynlacht, B.D., and Reinberg, D. (2008). Ezh1 and Ezh2 Maintain Repressive Chromatin through Different Mechanisms. Mol. Cell 32, 503-518.

Marine, J.C., Francoz, S., Maetens, M., Wahl, G., Toledo, F., and Lozano, G. (2006). Keeping p53 in check: Essential and synergistic functions of Mdm2 and Mdm4. Cell Death Differ. 13, 927-934.

Marinello, J., Chillemi, G., Bueno, S., Manzo, S.G., and Capranico, G. (2013). Antisense transcripts enhanced by camptothecin at divergent $\mathrm{CpG}$-island promoters associated with bursts of topoisomerase I-DNA cleavage complex and R-loop formation. Nucleic Acids Res. 41, 1011010123.

Martin, K., Trouche, D., Hagemeier, C., Sorensen, T.S., La Thangue, N.B., and Kouzarides, T. (1995). Stimulatuion of E2F1/DP1 transcriptional activity by MDM2 oncoprotein. Nature 375, 691694.

Masai, H., Matsumoto, S., You, Z., Yoshizawa-Sugata, N., and Oda, M. (2010). Eukaryotic chromosome DNA replication: where, when, and how? Annu. Rev. Biochem. 79, 89-130.

Mateu, M.G., and Fersht, A.R. (1999). Mutually compensatory mutations during evolution of the tetramerization domain of tumor suppressor p53 lead to impaired hetero-oligomerization. Proc. Natl. Acad. Sci. U. S. A. 96, 3595-3599.

Mateu, M.G., Sánchez Del Pino, M.M., and Fersht, A.R. (1999). Mechanism of folding and assembly of a small tetrameric protein domain from tumor suppressor p53. Nat. Struct. Biol. 6, 191-198.

Matijasevic, Z., Sluder, G., Gallant, J., and Jones, S.N. (2016). The Zn- finger domain of MdmX suppresses cancer progression by promoting genome stability in p53-mutant cells. Oncogenesis 5 , e262-10.

Matsuoka, S., Ballif, B. a, Smogorzewska, A., McDonald, E.R., Hurov, K.E., Luo, J., Bakalarski, C.E., Zhao, Z., Solimini, N., Lerenthal, Y., et al. (2007). ATM and ATR substrate analysis reveals extensive protein networks responsive to DNA damage. Science 316, 1160-1166.

Mattia, M., Gottifredi, V., McKinney, K., and Prives, C. (2007). p53-Dependent p21 mRNA elongation is impaired when DNA replication is stalled. Mol. Cell. Biol. 27, 1309-1320.

McIntosh, D., and Blow, J. (2012). Dormant Origins, the Licensing Checkpoint and the Response to Replicative Stresses. Cold Spring Harb. Perspect. ... 1-10.

McKenzie Duncan, I. (1982). Polycomblike: A gene that appears to be required for the normal expression of the bithorax and antennapedia gene complexes of Drosophila melanogaster. Genetics 102, 49-70. 
Méchali, M. (2010). Eukaryotic DNA replication origins: Many choices for appropriate answers. Nat. Rev. Mol. Cell Biol. 11, 728-738.

Meek, D.W., and Anderson, C.W. (1994). Post-translational modification of p53. Semin. Cancer Biol. 5, 203-210.

Mello, S.S., and Attardi, L.D. (2018). Deciphering p53 signaling in tumor suppression. Curr. Opin. Cell Biol. 51, 65-72.

Méndez, J., and Stillman, B. (2000). Chromatin association of human origin recognition complex, cdc6, and minichromosome maintenance proteins during the cell cycle: assembly of prereplication complexes in late mitosis. Mol. Cell. Biol. 20, 8602-8612.

Mendrysa, S.M., and Perry, M.E. (2000). The p53 tumor suppressor protein does not regulate expression of its own inhibitor, MDM2, except under conditions of stress. Mol. Cell. Biol. 20, 20232030.

Merrikh, H., MacHón, C., Grainger, W.H., Grossman, A.D., and Soultanas, P. (2011). Codirectional replication-transcription conflicts lead to replication restart. Nature 470, 554-558.

Meryet-Figuiere, M., Alaei-Mahabadi, B., Ali, M.M., Mitra, S., Subhash, S., Pandey, G.K., Larsson, E., and Kanduri, C. (2014). Temporal separation of replication and transcription during S-phase progression. Cell Cycle 13, 3241-3248.

Minsky, N., and Oren, M. (2004). The RING domain of Mdm2 mediates histone ubiquitylation and transcriptional repression. Mol. Cell 16,631-639.

Momand, J., Zambetti, G., Olson, D., George, D., and Levine, A. (1992). The mdm-2 oncogene product forms a complex with the p53 protein and inhi- bits p53-mediated transactivation.pdf.crdownload. Cell 69, 1237-1245.

Momand, J., Jung, D., Wilczynski, S., and Niland, J. (1998). The MDM2 gene amplification database. Nucleic Acids Res. 26, 3453-3459.

Momand, J., Villegas, A., and Belyi, V.A. (2011). The evolution of MDM2 family genes. Gene 486, 23-30.

Montagnoli, A., Valsasina, B., Brotherton, D., Troiani, S., Rainoldi, S., Tenca, P., Molinari, A., and Santocanale, C. (2006). Identification of Mcm2 phosphorylation sites by S-phase-regulating kinases. J. Biol. Chem. 281, 10281-10290.

Montagnoli, A., Valsasina, B., Croci, V., Menichincheri, M., Rainoldi, S., Marchesi, V., Tibolla, M., Tenca, P., Brotherton, D., Albanese, C., et al. (2008). A Cdc7 kinase inhibitor restricts initiation of DNA replication and has antitumor activity. Nat. Chem. Biol. 4, 357-365.

Montes de Oca Luna, R., Wagner, D.S., and Lozano, G. (1995). Rescue of early embryonic lethality in mdm2-deficient mice by deletion of p53. Nature 378, 203-206.

Morales, F., and Giordano, A. (2016). Overview of CDK9 as a target in cancer research. Cell Cycle $15,519-527$.

Moumen, A., Masterson, P., O'Connor, M.J., and Jackson, S.P. (2005). hnRNP K: An HDM2 target and transcriptional coactivator of p53 in response to DNA damage. Cell 123, 1065-1078.

Muller, P.A.J., Vousden, K.H., and Norman, J.C. (2011). P53 and Its Mutants in Tumor Cell Migration and Invasion. J. Cell Biol. 192, 209-218.

Nakano, K., Bálint, E., Ashcroft, M., and Vousden, K.H. (2000). A ribonucleotide reductase gene is a transcriptional target of p53 and p73. Oncogene 19, 4283-4289. 
Nguyen, H.D., Yadav, T., Giri, S., Saez, B., Graubert, T.A., and Zou, L. (2017). Functions of Replication Protein A as a Sensor of R Loops and a Regulator of RNaseH1. Mol. Cell 65, 832847.e4.

O'Carroll, D., Erhardt, S., Pagani, M., and Barton, S.C. (2001). The Polycomb -Group Gene Ezh2 Is Required for Early Mouse Development. Mol. Cell. Biol. 21, 4330-4336.

Oliner, J., Pietenpol, J., Thiagalingam, S., Gyuris, J., Kinzler, K.W., and Vogelstein, B. (1993). Onocoprotein Mdm2 conceals the activation domain of tumour suppressor p53. Nature 362, 857860 .

Oren, M., and Rotter, V. (2010). Mutant p53 gain-of-function in cancer. Cold Spring Harb. Perspect. Biol. 2, a001107.

Owens, J.C., Detweiler, C.S., and Li, J.J. (1997). CDC45 is required in conjunction with CDC7/DBF4 to trigger the initiation of DNA replication. Proc. Natl. Acad. Sci. U.S.A. 94, 1252112526.

Pannunzio, N.R., and Lieber, M.R. (2016). Dissecting the Roles of Divergent and Convergent Transcription in Chromosome Instability. Cell Rep. 14, 1025-1031.

Parajuli, S., Teasley, D.C., Murali, B., Jackson, J., Vindigni, A., and Stewart, S.A. (2017). Human ribonuclease $\mathrm{H} 1$ resolves $\mathrm{R}$-loops and thereby enables progression of the DNA replication fork. J. Biol. Chem. 292, 15216-15224.

Pasini, D., Bracken, A.P., Jensen, M.R., Denchi, E.L., and Helin, K. (2004). Suz12 is essential for mouse development and for EZH2 histone methyltransferase activity. EMBO J. 23, 4061-4071.

Pellegrino, S., Michelena, J., Teloni, F., Imhof, R., and Altmeyer, M. (2017). Replication-Coupled Dilution of H4K20me2 Guides 53BP1 to Pre-replicative Chromatin. Cell Rep. 19, 1819-1831.

Perry, M.E., Mendrysa, S.M., Saucedo, L.J., Tannous, P., and Holubar, M. (2000). p76 MDM2 Inhibits the Ability of p90 MDM2 to Destabilize p53. 275, 5733-5738.

Petermann, E., and Helleday, T. (2010). Pathways of mammalian replication fork restart. Nat. Rev. Mol. Cell Biol. 11, 683-687.

Petermann, E., Woodcock, M., and Helleday, T. (2010). Chk1 promotes replication fork progression by controlling replication initiation. Proc. Natl. Acad. Sci. U. S. A. 107, 16090-16095.

Petryk, N., Kahli, M., D’Aubenton-Carafa, Y., Jaszczyszyn, Y., Shen, Y., Silvain, M., Thermes, C., Chen, C.L., and Hyrien, O. (2016). Replication landscape of the human genome. Nat. Commun. 7, $1-13$.

Piunti, A., Rossi, A., Cerutti, A., Albert, M., Jammula, S., Scelfo, A., Cedrone, L., Fragola, G., Olsson, L., Koseki, H., et al. (2014). Polycomb proteins control proliferation and transformation independently of cell cycle checkpoints by regulating DNA replication. Nat. Commun. 1-7.

Pommier, Y. (2013). Drugging topoisomerases: lessons and challenges. ACS Chem. Biol. 8, 8295.

Prado, F., and Aguilera, A. (2005). Impairment of replication fork progression mediates RNA pollI transcription-associated recombination. EMBO J. 24, 1267-1276.

Raj, N., and Attardi, L.D. (2017). The transactivation domains of the p53 protein. Cold Spring Harb. Perspect. Med. 7.

Ray-Coquard, I., Blay, J.Y., Italiano, A., Le Cesne, A., Penel, N., Zhi, J., Heil, F., Rueger, R., Graves, B., Ding, M., et al. (2012). Effect of the MDM2 antagonist RG7112 on the P53 pathway in 
patients with MDM2-amplified, well-differentiated or dedifferentiated liposarcoma: An exploratory proof-of-mechanism study. Lancet Oncol. 13, 1133-1140.

Regairaz, M., Zhang, Y.W., Fu, H., Agama, K.K., Tata, N., Agrawal, S., Aladjem, M.I., and Pommier, Y. (2011). Mus81-mediated DNA cleavage resolves replication forks stalled by topoisomerase I-DNA complexes. J. Cell Biol. 195, 739-749.

Reijns, M.A.M., Rabe, B., Rigby, R.E., Mill, P., Astell, K.R., Lettice, L.A., Boyle, S., Leitch, A., Keighren, M., Kilanowski, F., et al. (2012). Enzymatic removal of ribonucleotides from DNA is essential for mammalian genome integrity and development. Cell 149, 1008-1022.

Renart, J., Reiser, J., and Stark, G.R. (1979). Transfer of proteins from gels to diazobenzyloxymethyl-paper and detection with antisera: a method for studying antibody specificity and antigen structure. Proc. Natl. Acad. Sci. 76, 3116-3120.

Riscal, R., Schrepfer, E., Arena, G., Cissé, M.Y., Bellvert, F., Heuillet, M., Rambow, F., Bonneil, E., Sabourdy, F., Vincent, C., et al. (2016). Chromatin-Bound MDM2 Regulates Serine Metabolism and Redox Homeostasis Independently of p53. Mol. Cell 62, 890-902.

Rivera, C., Gurard-Levin, Z.A., Almouzni, G., and Loyola, A. (2014). Histone lysine methylation and chromatin replication. Biochim. Biophys. Acta - Gene Regul. Mech. 1839, 1433-1439.

Roberts, R., and Crothers, D. (1992). Stability and properties of double and triple helices: dramatic effects of RNA or DNA backbone composition. Science (80-. ). 258, 1463-1466.

Rondinelli, B., Gogola, E., Yücel, H., Duarte, A.A., van de Ven, M., van der Sluijs, R., Konstantinopoulos, P.A., Jonkers, J., Ceccaldi, R., Rottenberg, S., et al. (2017). EZH2 promotes degradation of stalled replication forks by recruiting MUS81 through histone H3 trimethylation. Nat. Cell Biol.

Roth, J., Dobbelstein, M., Freedman, D.A., Shenk, T., and Levine, A.J. (1998). Nucleo-cytoplasmic shuttling of the hdm2 oncoprotein regulates the levels of the p53 protein via a pathway used by the human immunodeficiency virus rev protein. EMBO J. 17, 554-564.

Roy, S., Tomaszowski, K.H., Luzwick, J.W., Park, S., Li, J., Murphy, M., and Schlacher, K. (2018). p53 orchestrates DNA replication restart homeostasis by suppressing mutagenic RAD52 and POL $\theta$ pathways. Elife 7, 1-23.

Rozenfeld-Granot, G., Krishnamurthy, J., Kannan, K., Toren, A., Amariglio, N., Givol, D., and Rechavi, G. (2002). A positive feedback mechanism in the transcriptional activation of Apaf-1 by p53 and the coactivator Zac-1. Oncogene 21, 1469-1476.

Sablina, A. a, Budanov, A. V, Ilyinskaya, G. V, Larissa, S., Kravchenko, J.E., and Chumakov, P.M. (2005). The antioxidant function of the p53 tumor suppressor. Nat. Med. 11, 1306-1313.

Santos-Pereira, J.M., and Aguilera, A. (2015). R loops: New modulators of genome dynamics and function. Nat. Rev. Genet. 16, 583-597.

Schlacher, K., Christ, N., Siaud, N., Egashira, A., Wu, H., and Jasin, M. (2011). Double-strand break repair-independent role for BRCA2 in blocking stalled replication fork degradation by MRE11. Cell $145,529-542$.

Schuettengruber, B., and Cavalli, G. (2009). Recruitment of Polycomb group complexes and their role in the dynamic regulation of cell fate choice. Development 136, 3531-3542.

Schuettengruber, B., Chourrout, D., Vervoort, M., Leblanc, B., and Cavalli, G. (2007). Genome regulation by polycomb and trithorax proteins. Cell 128, 735-745. 
Schwartz, Y.B., and Pirrotta, V. (2013). A new world of Polycombs: Unexpected partnerships and emerging functions. Nat. Rev. Genet. 14, 853-864.

Schwartz, Y.B., Kahn, T.G., Nix, D.A., Li, X.Y., Bourgon, R., Biggin, M., and Pirrotta, V. (2006). Genome-wide analysis of Polycomb targets in Drosophila melanogaster. Nat. Genet. 38, 700-705.

Sdek, P., Ying, H., Zheng, H., Margulis, A., Tang, X., Tian, K., and Xiao, Z.X.J. (2004). The central acidic domain of MDM2 is critical in inhibition of retinoblastoma-mediated suppression of E2F and cell growth. J. Biol. Chem. 279, 53317-53322.

Sengupta, S., and Harris, C.C. (2005). p53: Traffic cop at the crossroads of DNA repair and recombination. Nat. Rev. Mol. Cell Biol. 6, 44-55.

Senturk, J.C., Bohlman, S., and Manfredi, J.J. (2017). Mdm2 selectively suppresses DNA damage arising from inhibition of topoisomerase II independent of p53. Oncogene 36, 6085-6096.

Shao, Z., Raible, F., Mollaaghababa, R., Guyon, J.R., Wu, C.T., Bender, W., and Kingston, R.E. (1999). Stabilization of chromatin structure by PRC1, a polycomb complex. Cell 98, 37-46.

Shapiro, A.L., Viñuela, E., and Maizel, J. V (1967). Molecular weight estimation of polypeptide chains by electrophoresis in SDS-polyacrylamide gels. Biochem. Biophys. Res. Commun. 28, 815820.

Sharp, D., and Kratowicz, S. (1999). Stabilization of the MDM2 Oncoprotein by Interaction with the Structurally Related MDMX Protein. J. Biol. ....

Shaw, N.N., and Arya, D.P. (2008). Recognition of the unique structure of DNA:RNA hybrids. Biochimie 90, 1026-1039.

Shiloh, Y. (2003). ATM and related protein kinases: Safeguarding genome integrity. Nat. Rev. Cancer 3, 155-168.

Shloush, J., Vlassov, J.E., Engson, I., Duan, S., Saridakis, V., Dhe-paganon, S., Raught, B., Sheng, Y., and Arrowsmith, C.H. (2011). Structural and functional comparison of the RING domains of two p53 E3 ligases, Mdm2 and Pirh2. J. Biol. Chem. 286, 4796-4808.

Shvarts, a, Steegenga, W.T., Riteco, N., van Laar, T., Dekker, P., Bazuine, M., van Ham, R.C., van der Houven van Oordt, W., Hateboer, G., van der Eb, a J., et al. (1996). MDMX: a novel p53binding protein with some functional properties of MDM2. EMBO J. 15, 5349-5357.

Sigalas, I., Calvert, A., Anderson, J., Neal, D., and Lunec, J. (1996). Alternatively spliced mdm2 transcripts with loss of p53 binding domain sequences: transforming ability and frequent detection in human cancer. Nat. Med. 2.

Simon, J. a, and Kingston, R.E. (2009). Mechanisms of polycomb gene silencing: knowns and unknowns. Nat. Rev. Mol. Cell Biol. 10, 697-708.

Sing, A., Pannell, D., Karaiskakis, A., Sturgeon, K., Djabali, M., Ellis, J., Lipshitz, H.D., and Cordes, S.P. (2009). A Vertebrate Polycomb Response Element Governs Segmentation of the Posterior Hindbrain. Cell 138, 885-897.

Smeenk, L., Van Heeringen, S.J., Koeppel, M., Van Driel, M.A., Bartels, S.J.J., Akkers, R.C., Denissov, S., Stunnenberg, H.G., and Lohrum, M. (2008). Characterization of genome-wide p53binding sites upon stress response. Nucleic Acids Res. 36, 3639-3654.

Smirnov, E., Borkovec, J., Kováčik, L., Svidenská, S., Schröfel, A., Skalníková, M., Švindrych, Z., Křižžek, P., Ovesný, M., Hagen, G.M., et al. (2014). Separation of replication and transcription domains in nucleoli. J. Struct. Biol. 188, 259-266. 
Smith, P., Krohn, R., and Hermanson, G. (1985). Measurement of Protein Using Bicinchoninic Acid. Anal. ... 85, 76-85.

Sollier, J., and Cimprich, K.A. (2015). Breaking bad: R-loops and genome integrity. Trends Cell Biol. 25, 514-522.

Sollier, J., Stork, C.T., García-Rubio, M.L., Paulsen, R.D., Aguilera, A., and Cimprich, K.A. (2014). Transcription-Coupled Nucleotide Excision Repair Factors Promote R-Loop-Induced Genome Instability. Mol. Cell 56, 777-785.

Srivatsan, A., Tehranchi, A., MacAlpine, D.M., and Wang, J.D. (2010). Co-orientation of replication and transcription preserves genome integrity. PLoS Genet. 6 .

Stiff, T., Walker, S.A., Cerosaletti, K., Goodarzi, A.A., Petermann, E., Concannon, P., O'Driscoll, M., and Jeggo, P.A. (2006). ATR-dependent phosphorylation and activation of ATM in response to UV treatment or replication fork stalling. EMBO J. 25, 5775-5782.

Stryer, L., Berg, J., and Tymoczko, J. (2002). Biochemistry (New York: W.H. Freeman).

Subramanian, D., and Griffith, J.D. (2005). P53 Monitors Replication Fork Regression By Binding To " Chickenfoot” Intermediates. J. Biol. Chem. 280, 42568-42572.

Sullivan, K.D., Galbraith, M.D., Andrysik, Z., and Espinosa, J.M. (2017). Mechanisms of transcriptional regulation by p53. Cell Death Differ. 25, 133-143.

Suzuki, Y., Holmes, J.B., Cerritelli, S.M., Sakhuja, K., Minczuk, M., Holt, I.J., and Crouch, R.J. (2010). An Upstream Open Reading Frame and the Context of the Two AUG Codons Affect the Abundance of Mitochondrial and Nuclear RNase H1. Mol. Cell. Biol. 30, 5123-5134.

Takisawa, H., Mimura, S., and Kubota, Y. (2000). Eukaryotic DNA replication : from pre-replication complex to initiation complex. Curr. Opin. Cell Biol. 12, 690-696.

Tanaka, H., Arakawa, H., Yamaguchi, T., Shiraishi, K., Fukuda, S., Matsui, K., and Takei, Y. (2000). A ribonucleotide reductase gene involved in a p53-dependent cell-cycle checkpoint for DNA damage.

Tanimura, S., Ohtsuka, S., Mitsui, K., Shirouzu, K., Yoshimura, a, and Ohtsubo, M. (1999). MDM2 interacts with MDMX through their RING finger domains. FEBS Lett. 447, 5-9.

Tasat, D., and Yakisich, J. (2010). Intra S-Phase Checkpoint. In DNA Damage Repair, Repair Mechanisms and Aging, A.E. Thomas, ed. (Nova Science Publishers Inc.), pp. 71-96.

Taylor, W.R., Agarwal, M.L., Agarwal, a, Stacey, D.W., and Stark, G.R. (1999). p53 inhibits entry into mitosis when DNA synthesis is blocked. Oncogene 18, 283-295.

Teufel, D.P., Bycroft, M., and Fersht, A.R. (2009). Regulation by phosphorylation of the relative affinities of the $\mathrm{N}$-terminal transactivation domains of p53 for p300 domains and Mdm2. Oncogene 28, 2112-2118.

Tomlinson, I., and Bodmer, W. (1999). Selection, the mutation rate and cancer: ensuring that the tail does not wag the dog. Nat. Med. 5, 11-12.

Tovy, A., Spiro, A., McCarthy, R., Shipony, Z., Aylon, Y., Allton, K., Ainbinder, E., Furth, N., Tanay, A., Barton, M., et al. (2017). p53 is essential for DNA methylation homeostasis in naïve embryonic stem cells, and its loss promotes clonal heterogeneity. Genes Dev. 31, 959-972.

Towbin, H., Staehelin, T., and Gordon, J. (1979). Electrophoretic transfer of proteins from polyacrylamide gels to nitrocellulose sheets: procedure and some applications. Proc. Natl. Acad. Sci. U. S. A. 76, 4350-4354. 
Trojer, P., and Reinberg, D. (2007). F fdacultative Heterochromatin: Is There a Distinctive Molecular Signature? Mol. Cell 28, 1-13.

Tuduri, S., Crabbé, L., Conti, C., Tourrière, H., Holtgreve-Grez, H., Jauch, A., Pantesco, V., De Vos, J., Thomas, A., Theillet, C., et al. (2009). Topoisomerase I suppresses genomic instability by preventing interference between replication and transcription. Nat. Cell Biol. 11, 1315-1324.

Uchida, C., Miwa, S., Kitagawa, K., Hattori, T., Isobe, T., Otani, S., Oda, T., Sugimura, H., Kamijo, T., Ookawa, K., et al. (2005). Enhanced Mdm2 activity inhibits pRB function via ubiquitindependent degradation. EMBO J. 24, 160-169.

Valente, L.J., Gray, D.H.D., Michalak, E.M., Pinon-Hofbauer, J., Egle, A., Scott, C.L., Janic, A., and Strasser, A. (2013). P53 Efficiently Suppresses Tumor Development in the Complete Absence of Its Cell-Cycle Inhibitory and Proapoptotic Effectors p21, Puma, and Noxa. Cell Rep. 3, 1339-1345.

Vassilev, L.T., Vu, B.T., Graves, B., Carvajal, D., Podlaski, F., Filipovic, Z., Kong, N., Kammlott, U., Lukacs, C., Klein, C., et al. (2004). In vivo activation of the p53 pathway by small-molecule antagonists of MDM2. Science 303, 844-848.

Vogelstein, B., Lane, D., and Levine, a J. (2000). Surfing the p53 network. Nature 408, 307-310.

Wahba, L., Amon, J.D., Koshland, D., and Vuica-Ross, M. (2011). RNase H and Multiple RNA Biogenesis Factors Cooperate to Prevent RNA:DNA Hybrids from Generating Genome Instability. Mol. Cell 44, 978-988.

Wang, D., and Lippard, S.J. (2005). Cellular processing of platinum anticancer drugs. Nat. Rev. Drug Discov. 4, 307-320.

Wang, H., Wang, L., Erdjument-Bromage, H., Vidal, M., Tempst, P., Jones, R.S., and Zhang, Y. (2004). Role of histone H2A ubiquitination in Polycomb silencing. Nature 431, 873-878.

Wang, X., Wang, J., and Jiang, X. (2011). MdmX protein is essential for Mdm2 protein-mediated p53 polyubiquitination. J. Biol. Chem. 286, 23725-23734.

Wen, W., Peng, C., Kim, M.O., Ho Jeong, C., Zhu, F., Yao, K., Zykova, T., Ma, W., Carper, A., Langfald, A., et al. (2014). Knockdown of RNF2 induces apoptosis by regulating MDM2 and p53 stability. Oncogene 33, 421-428.

Westover, K.D. (2004). Structural Basis of Transcription: Separation of RNA from DNA by RNA Polymerase II. Science (80-. ). 303, 1014-1016.

Wienken, M., Dickmanns, A., Nemajerova, A., Kramer, D., Najafova, Z., Weiss, M., Karpiuk, O., Kassem, M., Zhang, Y., Lozano, G., et al. (2016). MDM2 Associates with Polycomb Repressor Complex 2 and Enhances Stemness-Promoting Chromatin Modifications Independent of p53. Mol. Cell 61, 68-83.

Wienken, M., Moll, U.M., and Dobbelstein, M. (2017). Mdm 2 as a chromatin modifier. 1-7.

Willis, A., Jung, E.J., Wakefield, T., and Chen, X. (2004). Mutant p53 exerts a dominant negative effect by preventing wild-type p53 from binding to the promoter of its target genes. Oncogene 23, 2330-2338.

Woodward, A.M., Göhler, T., Luciani, M.G., Oehlmann, M., Ge, X., Gartner, A., Jackson, D. a, and Blow, J.J. (2006). Excess Mcm2-7 license dormant origins of replication that can be used under conditions of replicative stress. J. Cell Biol. 173, 673-683.

Wu, H., Lima, W.F., and Crooke, S.T. (2001). Investigating the Structure of Human RNase H1 by Site-directed Mutagenesis. J. Biol. Chem. 276, 23547-23553. 
Wu, R., Wang, Z., Zhang, H., Gan, H., and Zhang, Z. (2017). H3K9me3 demethylase Kdm4d facilitates the formation of pre-initiative complex and regulates DNA replication. Nucleic Acids Res. 45, 169-180.

Wu, X., Bayle, J.H., Olson, D., and Levine, a J. (1993). The p53-mdm-2 autoregulatory feedback loop. Genes Dev. 7, 1126-1132.

Wylie, A., Jones, A.E., D’Brot, A., Lu, W.J., Kurtz, P., Moran, J. V., Rakheja, D., Chen, K.S., Hammer, R.E., Comerford, S.A., et al. (2016). P53 Genes Function To Restrain Mobile Elements. Genes Dev. 30, 64-77.

Xeros, N. (1962). Deoxyriboside control and synchronization of mitosis. Nature 194, 682-683.

Xiao, Z.X., Chen, J., Levine, A.J., Modjtahedi, N., Xing, J., Sellers, W.R., and Livingston, D.M. (1995). Interaction between the retinoblastoma protein and the oncoprotein MDM2. Nature 375, 694-698.

Xie, L., Gazin, C., Park, S.M., Zhu, L.J., Debily, M. anne, Kittler, E.L.W., Zapp, M.L., Lapointe, D., Gobeil, S., Virbasius, C.M., et al. (2012). A Synthetic Interaction Screen Identifies Factors Selectively Required for Proliferation and TERT Transcription in p53-Deficient Human Cancer Cells. PLoS Genet. 8.

Yadav, P., Harcy, V., Argueso, J.L., Dominska, M., Jinks-Robertson, S., and Kim, N. (2014). Topoisomerase I Plays a Critical Role in Suppressing Genome Instability at a Highly Transcribed G-Quadruplex-Forming Sequence. PLoS Genet. 10.

Yang, J.Y., Zong, C.S., Xia, W., Yamaguchi, H., Ding, Q., Xie, X., Lang, J.Y., Lai, C.C., Chang, C.J., Huang, W.C., et al. (2008). ERK promotes tumorigenesis by inhibiting FOXO3a via MDM2mediated degradation. Nat. Cell Biol. 10, 138-148.

Yeo, C.Q.X., Alexander, I., Lin, Z., Lim, S., Aning, O.A., Kumar, R., Sangthongpitag, K., Pendharkar, V., Ho, V.H.B., and Cheok, C.F. (2016). P53 Maintains Genomic Stability by Preventing Interference between Transcription and Replication. Cell Rep. 15, 132-146.

Zee, B.M., Levin, R.S., Xu, B., LeRoy, G., Wingreen, N.S., and Garcia, B. a (2010). In vivo residuespecific histone methylation dynamics. J. Biol. Chem. 285, 3341-3350.

Zhang, Z., Wang, H., Li, M., Rayburn, E.R., Agrawal, S., and Zhang, R. (2005). Stabilization of E2F1 protein by MDM2 through the E2F1 ubiquitination pathway. Oncogene 24, 7238-7247.

Zhao, H., and Piwnica-Worms, H. (2001). ATR-Mediated Checkpoint Pathways Regulate Phosphorylation and Activation of ATR-Mediated Checkpoint Pathways Regulate Phosphorylation and Activation of Human Chk1. Mol. Cell. Biol. 21, 4129-4139.

Zhao, R., Gish, K., Murphy, M., Yin, Y., Notterman, D., Hoffman, W.H., Tom, E., Mack, D.H., and Levine, A.J. (2000). Analysis of p53-regulated gene expression patterns using oligonucleotide arrays. Genes \&amp; Dev. 14, 981-993.

Zhou, B.-B.S., and Elledge, S.J. (2000). Checkpoints in perspective. Nature 408, 433-439.

Zofall, M., Smith, D.R., Mizuguchi, T., Dhakshnamoorthy, J., and Grewal, S.I.S. (2016). Taz1Shelterin Promotes Facultative Heterochromatin Assembly at Chromosome-Internal Sites Containing Late Replication Origins. Mol. Cell 62, 862-874. 


\section{Acknowledgements}

I would like to thank Matthias Dobbelstein for his supportive and encouraging supervision of my project. I am grateful for having been given the opportunity to establish my own project during my Master's time and continue it during my doctoral studies. Throughout this time, Matthias has guided me scientifically, encouraged my personal development and made my stay in the lab very successful and enjoyable.

I would also like to thank my thesis advisory committee members Halyna Shcherbata and Steven Johnsen for fruitful discussions and valuable input on my work during annual meetings. Furthermore, I thank Heidi Hahn, Roland Dosch and Nuno Raimundo for assessing my work in the extended thesis committee.

My four years stay in the Department of Molecular Oncology has been a great time in company of many inspiring people. I thank all members of the Molecular Oncology, Tumour Epigenetics, and Molecular and Experimental Pneumology groups for creating a pleasant working environment, providing input during our joint seminars and being supportive in all matters.

In particular, I would like to thank Magdalena Wienken and Antje Dickmanns for giving me such a good start in the lab; Ramona and Muriel for their help regarding all animal-related questions and experiments; our office crew with all past and present members for scientific discussions as well as fun times - Anusha, Josephine, Florian, Vijaya, Simon, Robyn and Feda.

I would also like to thank everyone that has contributed to this project; in particular Sabrina, Mascha, Leonie, Yizhu, Kai, Valentina and Anna for their great help and contributions in completing this part of the project and taking it further in the future.

Scientific work lives on the exchange of ideas and concepts with fellow scientists. I am very grateful for the $\mathrm{PhD}$ fellowship received from the Boehringer Ingelheim Fonds as well as funding from both BIF and the IMPRS Molecular Biology Program that allowed me to attend many conferences and scientific meetings to discuss my work. I would like to thank the BIF team with Claudia, Anja and Sandra as well as the MolBio team of Steffen and Kerstin for their constant support and the great retreats filled with science, hiking and a lot of fun.

Last but definitely not least, I would like to thank my family and friends that have been an important anchor during times of highs and lows of this work. I am incredibly thankful for your understanding, patience and encouragement during the last couple of years.

Special thanks go to Anusha, Josephine, Kai, and Nora for proofreading my thesis. 


\section{Abbreviations}

\begin{tabular}{|c|c|c|c|}
\hline${ }^{\circ} \mathrm{C}$ & Degree Celsius & Gem & Gemcitabine \\
\hline $4-\mathrm{OHT}$ & 4-hydroxy-tamoxifen & GFP & green fluorescent protein \\
\hline $5-\mathrm{FU}$ & 5-fluoro-uracil & Glu & glutamate \\
\hline AID & activation-induced cytidine deaminase & $\mathrm{h}$ & hour \\
\hline APS & ammonium persulfate & HOAK119uh1 & histone $2 \mathrm{~A}$ lysine 119 \\
\hline AQR & aquarius & 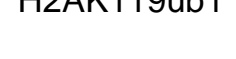 & monoubiquitination \\
\hline ATM & ataxia telangiectasia mutated & $\mathrm{H} 2 \mathrm{AX}$ & histone variant $2 \mathrm{AX}$ \\
\hline ATR & ATM- and Rad3-related & H3K27me3 & histone 3 lysine 27 trimethylation \\
\hline ATRIP & ATR interacting protein & & heterogeneous nuclear \\
\hline BER & base excision repair & . & ribonucleoprotein \\
\hline bp & base pair & $\mathrm{HR}$ & homologous recombination \\
\hline BrdU & bromodeoxyuridine & HRP & horseradish peroxidase \\
\hline BSA & bovine serum albumine & $\mathrm{HU}$ & hydroxyurea \\
\hline CBP & CREB-binding protein & IDD & intrinsically disordered domain \\
\hline CDK & cyclin-dependent kinase & IdU & iododeoxyuridine \\
\hline cDNA & complementary DNA & iPSC & induced pluripotent stem cell \\
\hline CFS & common fragile site & $\mathrm{kb}$ & kilobases \\
\hline $\mathrm{CHK}$ & checkpoint kinase & $\mathrm{kd}$ & knockdown \\
\hline CldU & chlorodeoxyuridine & $\mathrm{kDa}$ & kilodalton \\
\hline $\mathrm{CO} 2$ & carbondioxide & M & molar \\
\hline $\mathrm{CpG}$ & cytosine phosphatidyl guanine & Mdm2/MDM2 & murine double minute 2 \\
\hline $\mathrm{Ct}$ & cycle threshold & MEF & mouse embryonic fibroblast \\
\hline CTD & C-terminal domain & $\mathrm{MetOH}$ & methanol \\
\hline ctrl & control & $\mathrm{mg}$ & milligram \\
\hline DDR & DNA damage response & $\min$ & Minute \\
\hline $\mathrm{dFdC}$ & 2',2'-diofluoro-deoxycytidine & $\mathrm{ml}$ & millilitre \\
\hline DHX9 & DEAH box protein 9 & $\mathrm{mM}$ & millimolar \\
\hline DMEM & Dulbecco's modified Eagle medium & mRNA & messenger RNA \\
\hline DMSO & dimethylsulfoxide & $\mathrm{n}$ & sample size \\
\hline DNA & deoxyribonucleic acid & NER & nucleotide excision repair \\
\hline dNTP & deoxyribonucleotide triphosphate & NES & nuclear export signal \\
\hline DSB & double strand break & NHEJ & non-homologous end joining \\
\hline dsDNA & double standed DNA & NLS & nuclear localisation signal \\
\hline EdU & 5-ethynyl-2'-deoxyuridine & $\mathrm{nm}$ & nanometer \\
\hline $\mathrm{EtOH}$ & ethanol & $\mathrm{nM}$ & nanomolar \\
\hline $\mathrm{EZH} 2$ & enhancer of zeste homologue 2 & $\mathrm{nM}$ & nanomolar \\
\hline FCS & fetal calf serum & NoLS & nucleolar localisation signal \\
\hline $\mathrm{fl}$ & floxed & NOS & reactive nitrogen species \\
\hline
\end{tabular}




\begin{tabular}{|c|c|c|c|}
\hline OE & \multicolumn{2}{|l|}{ overexpression } & \multirow{2}{*}{$\begin{array}{l}\text { Trithorax } \\
\text { senataxin }\end{array}$} \\
\hline ORC & origin recognition proteins & SETX & \\
\hline p53RE & p53 response element & siRNA & small interfering ribonucleic acid \\
\hline PBS & phosphate buffered saline & ssDNA & single stranded DNA \\
\hline PcG & Polycomb group & SUZ12 & suppressor of zeste homologue 12 \\
\hline PCNA & Proliferating cell nuclear antigen & TAD & trans-activation domain \\
\hline PIC & pre-initiation complex & TAR & transcription-associated repair \\
\hline PRC & polycomb repressor complex & TBP & TATA-binding protein \\
\hline pre-mRNA & pre-messenger RNA & TBS & Tris buffered saline \\
\hline \multirow[t]{2}{*}{ pre-RC } & pre-replication complex & TBS-T & Tris buffered saline + Tween 20 \\
\hline & \multicolumn{2}{|c|}{ quantitative real time polymerase chainTEMED } & tetramethylethylenediamine \\
\hline qRT-PCR & reaction & TF & transcription factor \\
\hline RING & really interesting new gene & Thr & threonine \\
\hline RNA & ribonucleic acid & TLS & translesion synthesis \\
\hline \multirow{2}{*}{$\begin{array}{l}\text { RNA Pol } \\
\text { (RNAP) }\end{array}$} & \multirow{2}{*}{ RNA polymerase } & Tris & trisamine \\
\hline & & wt & wildtype \\
\hline RNase & ribonuclease & $\mathrm{XPF}$ & xeroderma pigmentosum factor $F$ \\
\hline RNR & ribonucleotide reductase & XPG & xeroderma pigmentosum factor $\mathrm{G}$ \\
\hline ROS & reactive oxygen species & $\mu g$ & microgram \\
\hline RPA & replication protein $A$ & $\mu l$ & microlitre \\
\hline rpm & Rounds per minute & $\mu \mathrm{M}$ & micromolar \\
\hline SDS & sodium dodecyl sulfate & & \\
\hline SDS-PAGE & $\begin{array}{l}\text { SDS-polyacrylamide gel } \\
\text { electrophoresis }\end{array}$ & & \\
\hline Ser & serine & & \\
\hline SET & Su(var)3-9, Enhancer-of-zeste an & & \\
\hline
\end{tabular}




\section{List of Figures}

Fig.II.1.1 Domain structure of p53.

Fig.Il.1.2 Domain architecture of MDM2.

Fig.II.1.3 The auto-regulatory feedback loop of p53 and MDM2 .........................................................

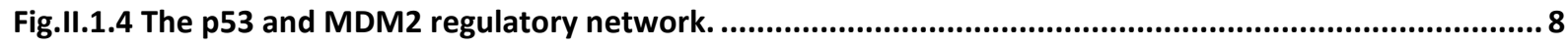

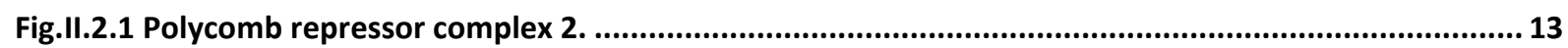

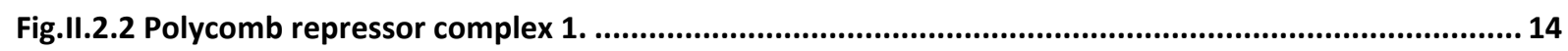

Fig.II.3.1 The DNA damage response (DDR) - a signalling cascade.........................................................16

Fig.II.3.2 Formation of the pre-replication complex. ............................................................................17

Fig.II.3.3 Schematic diagram of a replication fork (simplified). ................................................................. 18

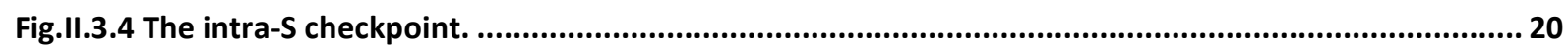

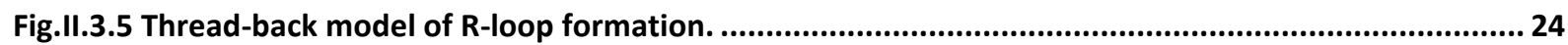

Fig. IV.3.1 Representative images of DNA fibers and a schematic diagram of structures observed in fiber

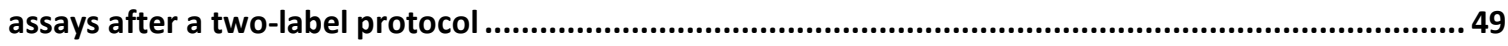

Fig. IV.3.2 Representative image of a fiber observed in the fork stalling assay with seven alternating labels of

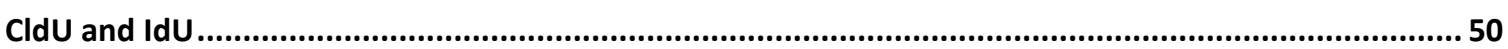

Fig. V.I.1 p53-mediated induction of genes during S-phase. .......................................................57

Fig.V.I.2 Enhanced replication fork progression upon p53 activation. ...................................................59

Fig.V.I.3 Increased replicative stress upon p53 depletion, and upon depletion of Mdm2 from p53-deficient cell.

Fig.V.I.4 p53-supported DNA replication in murine embryonic fibroblasts. ..............................................66

Suppl. Fig. V.I.7.1 p53-responsive gene expression during S phase. Related to Fig. V.I.1 ............................... 75

Suppl. Fig. V.I.7.2 Impact of Nutlin on DNA replication in U2OS cells. Related to Fig. V.I.2 ........................... 76

Suppl. Fig. V.I.7.3. Impact of Nutlin on replication processivity in U2OS cells. Related to Fig. V.I.2 ................ 78

Suppl. Fig. V.I.7.4 Depletion of p53 impairs DNA replication. Related to Fig. V.I.3.......................................80

Suppl. Fig. V.I.7.5 Depletion of p53 does not alter fork restart or reversal; Mdm2 mediates fork progression .

Related to Fig. V.I.3.

Suppl. Fig. V.I.7.6 P53 depletion decreases DNA replication fork progression in murine embryonic fibroblasts

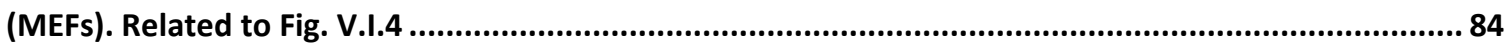

Suppl. Fig. V.I.7.7 Impact of p53 on DNA replication in thymocytes. Related to Fig. V.I.5 ..............................86

Fig.V.II.1 Compromised DNA replication fork progression upon depletion of Mdm2 or PRC2 members.........93

Fig.V.II.2 Decreased fork progression upon RNF2 depletion or Bmi1 inhibition......................................... 95

Fig.V.II.3 Requirement for the Ring finger domain of $\mathrm{Mdm} 2$ to support replication fork progression. ..........99

Fig.V.II.4 Mutual rescue of fork progression in Mdm2- and RNF2-depleted cells....................................... 101

Fig.V.Il.5 Mdm2 depletion increases replicative stress and R-loop formation. .......................................... 103

Fig. V.II.6 Rescue of DNA replication by CDK9 inhibitors upon depletion of Mdm2 or RNF2 ..................... 105

Fig. V.II.7 Restored DNA replication by removing DNA/RNA hybrids in cells depleted of Mdm2 or RNF2..... 107 
Suppl. Fig. V.II.7.1 Compromised DNA replication fork progression upon depletion of Mdm2 or PRC2

members. Related to Fig. V.II. 1.

Suppl. Fig. V.II.7.2 Decreased fork progression upon RNF2 depletion or Bmi1 inhibition Related to Fig. V.II.2.

Suppl. Fig. V.II.7.3 Requirement for the Ring finger domain of Mdm2 to support replication fork progression.

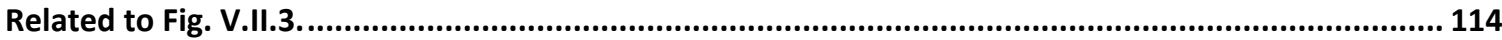

Suppl. Fig. V.II.7.4 Mutual rescue of for progression by Mdm2 and RNF2. Related to Fig. V.II.4. 115

Suppl. Fig. V.II.7.5 Mdm2 depletion increases replicative stress and R-loop formation. Related to Fig. V.II.5.

Suppl. Fig. V.II.7.6 Rescue of DNA replication by CDK9 inhibitors upon depletion of Mdm2 or RNF2. Related to

Fig. V.II.6. 117

Suppl. Fig. V.II.7.7 Restored DNA replication by removing DNA/RNA hybrids in cells depleted of Mdm2 or RNF2. Related to Fig. V.II.7.

Fig.VI.1 The p53-MDM2 network facilitates replication fork progression by preventing R-loop formation. . 120

Fig.VI.1 Fiber length is determined by both fork progression and processivity. cf. Suppl. Fig. V.I.7.3E 123

\section{List of Tables}

Table IV.1 Cell culture media 39

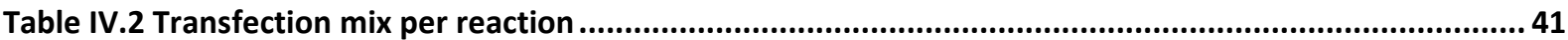

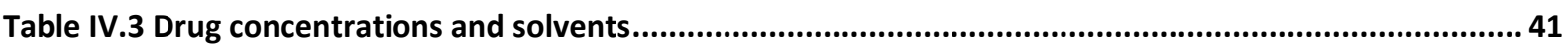

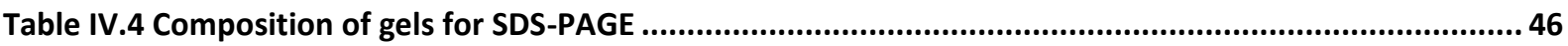

Table IV.5 Primer sequences for gene expression studies in human cells ...............................................48 


\section{Affidavit}

Herewith I declare that the PhD Thesis entitled "The tumour suppressor p53 as a supporter of DNA replication" was written independently and with no other sources and aids than quoted.

Ina Klusmann

Göttingen, July 2018 